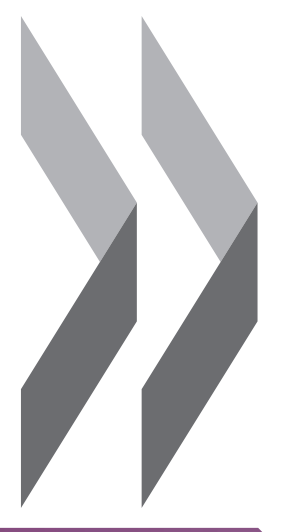

\title{
Panorama de l'entrepreneuriat 2017
}

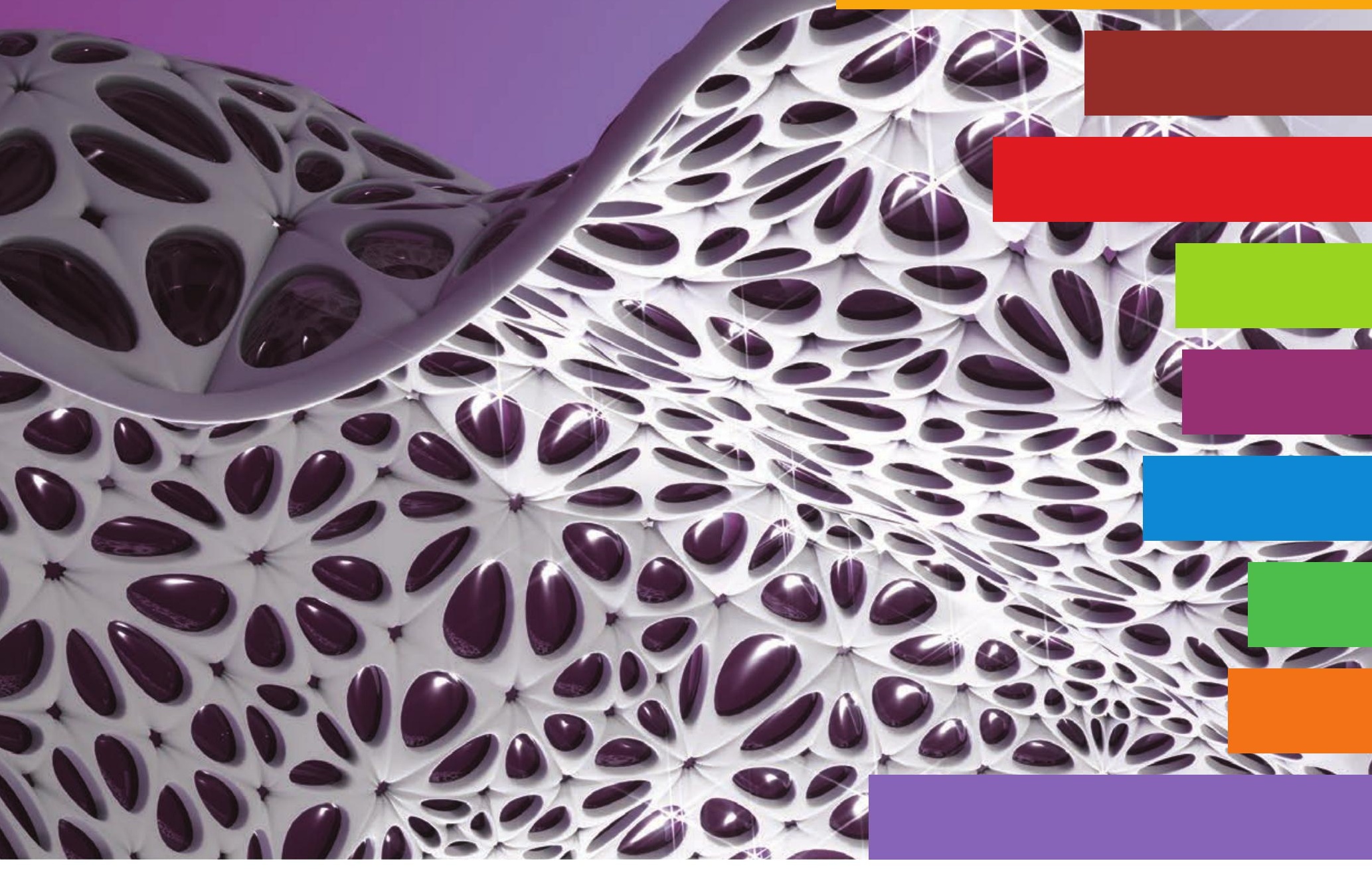

Q) OCDE 



\section{Panorama \\ de l'entrepreneuriat 2017}

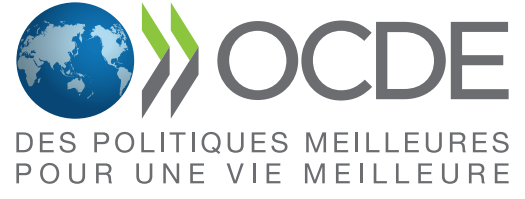


Cet ouvrage est publié sous la responsabilité du Secrétaire général de l'OCDE. Les opinions et les interprétations exprimées ne reflètent pas nécessairement les vues officielles des pays membres de l'OCDE.

Ce document, ainsi que les données et cartes qu'il peut comprendre, sont sans préjudice du statut de tout territoire, de la souveraineté s'exerçant sur ce dernier, du tracé des frontières et limites internationales, et du nom de tout territoire, ville ou région.

Merci de citer cet ouvrage comme suit :

OCDE (2018), Panorama de l'entrepreneuriat 2017, Éditions OCDE, Paris.

http://dx.doi.org/10.1787/entrepreneur_aag-2017-fr

ISBN 978-92-64-28891-1 (imprimé)

ISBN 978-92-64-28892-8 (PDF)

ISBN 978-92-64-28894-2 (HTML)

ISBN 978-92-64-28893-5 (epub)

Série : Panorama de l'entrepreneuriat

ISSN 2226-695X (imprimé)

ISSN 2226-6968 (en ligne)

Les données statistiques concernant Israël sont fournies par et sous la responsabilité des autorités israéliennes compétentes. L'utilisation de ces données par l'OCDE est sans préjudice du statut des hauteurs du Golan, de JérusalemEst et des colonies de peuplement israéliennes en Cisjordanie aux termes du droit international.

Crédits photo : Couverture ๑ Yuriy Bel?mesov/Shutterstock.com; Chapters: ๑ Philippe Mairesse/Devizu.

Les corrigenda des publications de l'OCDE sont disponibles sur : www.oecd.org/about/publishing/corrigenda.htm.

(c) OCDE 2018

La copie, le téléchargement ou l'impression du contenu OCDE pour une utilisation personnelle sont autorisés. Il est possible d'inclure des extraits de publications, de bases de données et de produits multimédia de l'OCDE dans des documents, présentations, blogs, sites internet et matériel pédagogique, sous réserve de faire mention de la source et du copyright. Toute demande en vue d'un usage public ou commercial ou concernant les droits de traduction devra être adressée à rights@oecd.org. Toute demande d'autorisation de photocopier une partie de ce contenu à des fins publiques ou commerciales devra être soumise au Copyright Clearance Center (CCC), info@copyright.com, ou au Centre français d'exploitation du droit de copie (CFC), contact@cfcopies.com. 


\section{Avant-Propos}

L e recueil d'indicateurs de l'entrepreneuriat présenté dans le Panorama de l'entrepreneuriat est la résultante du Programme d'indicateurs de l'entrepreneuriat OCDE-Eurostat (PIE). Lancé en 2006, ce programme incarne les premiers efforts déployés pour recueillir et publier des données internationales sur l'entrepreneuriat à partir de sources statistiques publiques officielles. D'emblée, l'une des priorités a été de réduire au minimum les coûts de compilation induits par l'établissement des indicateurs, pour les offices statistiques nationaux, ce qui explique que le programme privilégie l'exploitation des sources de données existantes.

Le but du PIE est de fournir des indicateurs utiles pour éclairer l'élaboration des politiques. Dans cette optique, une attention particulière est portée aux besoins d'information, en particulier à la nécessité de disposer en temps utile de renseignements sur la situation des petites entreprises. À cette fin, le premier chapitre du Panorama de l'entrepreneuriat passe en reuue les tendances récentes de l'entrepreneuriat s'agissant des créations et faillites d'entreprises, ainsi que du travail indépendant.

La présente édition a été élaborée au sein de la Division des statistiques du commerce et de la compétitivité de la Direction des statistiques de l'OCDE, par Liliana Suchodolska, Gueram Sargsyan, Belén Zinni et Frédéric Parrot. Nadim Ahmad et Mariarosa Lunati ont supervisé les opérations et assuré la direction de la publication.

Nous remercions tout particulièrement Eurostat, les experts des offices statistiques nationaux d'Afrique du Sud, d'Allemagne, d'Australie, d'Autriche, de Belgique, du Brésil, du Canada, du Chili, de Colombie, de Corée, du Danemark, d'Espagne, d'Estonie, des États-Unis, de la Fédération de Russie, de Finlande, de France, de Hongrie, d'Islande, d'Israël, d'Italie, du Japon, de Lettonie, de Lituanie, du Luxembourg, du Mexique, de Norvège, de Nouvelle-Zélande, des Pays-Bas, de Pologne, du Portugal, de République slovaque, de République tchèque, de Roumanie, du Royaume-Uni, de Slovénie, de Suède et de Suisse, ainsi que Cornelius Mueller d'Invest Europe, Darrell Pinto de la CVCA (Association canadienne du capital de risque et d'investissement), Maryam Haque de la National America Venture Capital Association of the United States et Kyle Stanford de Pitchbook pour leur aide et leurs conseils en matière de statistiques sur le capital-risque. 



\section{Table des matières}

Résumé $\ldots \ldots \ldots \ldots \ldots \ldots \ldots \ldots \ldots \ldots \ldots \ldots \ldots \ldots \ldots \ldots \ldots \ldots \ldots \ldots \ldots, 7$

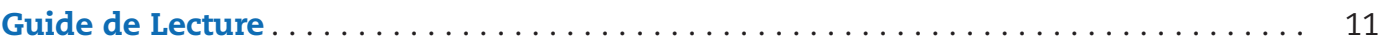

1. Évolutions récentes de l'entrepreuneuriat $\ldots \ldots \ldots \ldots \ldots \ldots \ldots \ldots \ldots \ldots \ldots$

Rebond des créations d'entreprises dans la plupart des pays . ............ 18

Le nombre de faillites est revenu à son niveau d'avant la crise . . . . . . . . . 25

Le travail indépendant a évolué différemment selon les pays . . . . . . . . . . 27

Le numérique ouvre de nouvelles perspectives et de nouveaux marchés qui favorisent la croissance de l'entrepreneuriat............... 30

2. Structure et performance de la population des entreprises............ 37

Entreprises par classe de taille . . . . . . . . . . . . . . . . . . . 38

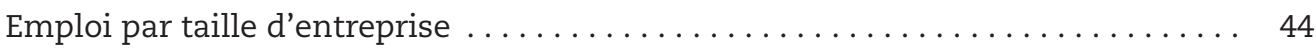

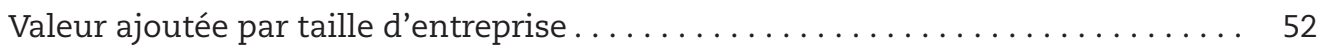

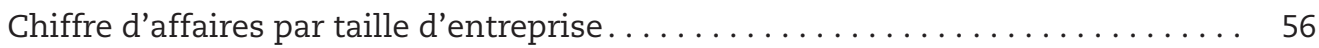

Rémunération des salariés par taille d'entreprise $\ldots \ldots \ldots \ldots \ldots \ldots \ldots . \ldots$

3. Productivité par taille d'entreprise $\ldots \ldots \ldots \ldots \ldots \ldots \ldots \ldots \ldots \ldots \ldots \ldots \ldots \ldots \ldots \ldots \ldots$

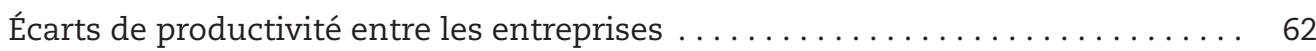

Croissance de la productivité par taille d'entreprise . . . . . . . . . . . . 64

Productivité et écarts de salaire entre les entreprises ................. 68

4. Dynamisme des entreprises et création d'emplois $\ldots \ldots \ldots \ldots \ldots \ldots \ldots \ldots . \ldots 3$

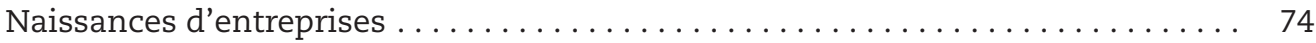

Décès d'entreprises ..................................... 80

Taux de renouvellement. . . . . . . . . . . . . . . . . . . . . 86

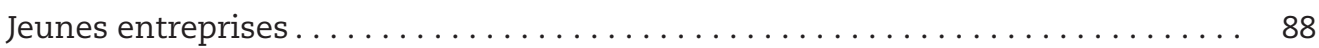

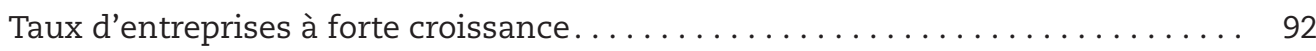

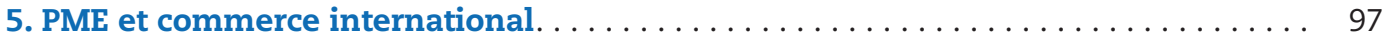

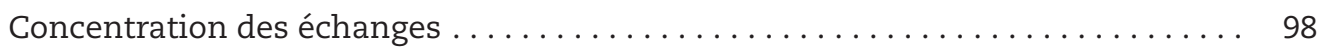

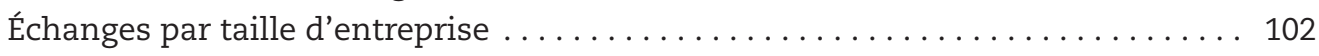

PME et proximité du marché .............................. 106

Échanges par type de contrôle capitalistique des entreprises . . . . . . . . . . . 108

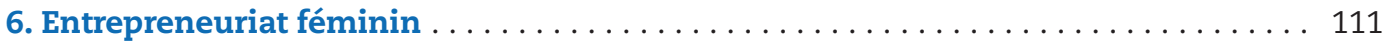

Taux de travail indépendant : différences entre femmes et hommes......... . 112

Revenus du travail indépendant . . . . . . . . . . . . . . . . . 118

Attitude à l'égard de l'entrepreneuriat. . . . . . . . . . . . . . . . . . 120 
7. Capital-risque . . . . . . . . . . . . . . . . . . . . . . . . . . 125

Investissements de capital-risque $\ldots \ldots \ldots \ldots \ldots \ldots \ldots \ldots \ldots \ldots \ldots \ldots$

Investissements de capital-risque par entreprise bénéficiaire . . . . . . . . . . 128

Investissements de capital-risque par secteur . . . . . . . . . . . . . 132

Annexe A. Sources des données sur les indicateurs actualisés de l'entrepreneuriat. . 135

Annexe B. Liste des indicateurs des déterminants entrepreneuriaux . . . . . . . . . 140

Annexe C. Comparabilité internationale des données sur le capital-risque . . . . . . 145

\title{
Suivez les publications de l'OCDE sur:
}

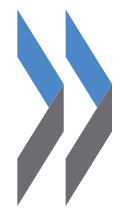

http://twitter.com/OECD_Pubs

ifttp://www.facebook.com/OECDPublications

in. http://www.linkedin.com/groups/OECD-Publications-4645871

Toube http://www.youtube.com/oecdilibrary

OECD http://www.oecd.org/oecddirect/

\section{Ce livre contient des...}

\author{
StatLinks 켄ㄴ \\ Accédez aux fichiers Exceløà à partir des livres imprimés !
}

En bas des tableaux ou graphiques de cet ouvrage, vous trouverez des StatLinks. Pour télécharger le fichier Excel@ correspondant, il vous suffit de retranscrire dans votre navigateur Internet le lien commençant par : http://dx.doi.org, ou de cliquer sur le lien depuis la version PDF de l'ouvrage. 


\section{Résumé}

$\mathrm{D}$

ans la plupart des pays de l'OCDE pour lesquels on dispose de données, le nombre de créations d'entreprises continue de se redresser, et dans de nombreux pays, il est supérieur aux points hauts atteints avant la crise, ce qui laisse à penser que le mouvement de baisse séculaire des taux de création d'entreprises mis en évidence par certaines études serait en train de s'infléchir.

L'amélioration observée sur le front des faillites étaye les signes d'embellie concernant l'environnement des entreprises. À la fin de 2016, le nombre de faillites était revenu à son niveau d'avant la crise, voire en deçà, dans la plupart des pays. Et même dans les pays où le niveau des faillites demeurait plus élevé qu'en 2007, en particulier en Espagne, en Islande et en Italie, les évolutions observées au début de l'année 2017 commencent à laisser entrevoir des améliorations.

\section{Les services ont été un moteur important de création d'entreprises}

Dans tous les pays de l'OCDE, les taux de création d'entreprises dans les services ont été plus élevés que dans l'industrie, contribuant à hauteur de deux tiers environ à l'ensemble des créations d'emplois imputables aux nouvelles entreprises en 2014. Néanmoins, dans la plupart des économies, les nouvelles entreprises industrielles ont représenté moins de $15 \%$ des créations d'emplois. En outre, entre 2008 et 2014, l'emploi dans le secteur manufacturier a diminué dans tous les pays de l'OCDE sauf deux : le Luxembourg et l'Allemagne.

Les taux d'emploi se sont également redressés dans de nombreux pays et, dans la plupart, les petites et moyennes entreprises (PME), jeunes et anciennes, ont été le principal moteur de la croissance de l'emploi. Au sein du secteur manufacturier, la croissance de l'emploi dans les grandes entreprises de la zone euro, qui ont été moins durement touchées par la crise que les PME, est restée plus rapide que dans ces dernières. Aux États-Unis, ce sont les grandes entreprises du secteur des services qui ont tiré la croissance de l'emploi après la crise.

Les écarts de productivité entre grandes et petites entreprises varient considérablement suivant les pays et les secteurs. Le plus souvent, toutefois, les écarts de productivité sont nettement plus modestes dans le secteur des services que dans le secteur manufacturier. En règle générale, plus cet écart de productivité est important, plus l'écart de salaire l'est aussi (en Allemagne, par exemple, les salaires versés par les grandes entreprises étaient supérieurs de plus de $50 \%$ à ceux payés par les entreprises de taille moyenne, et représentaient plus du double des salaires versés par les petites et micro- entreprises), ce qui signifie qu'une expansion des activités du secteur des services peut contribuer à resserrer la distribution des salaires dans l'ensemble de l'économie. 
Renforcer l'entrepreneuriat dans le secteur des services pourrait également contribuer à la poursuite de la réduction des inégalités entre hommes et femmes, étant donné que les femmes sont représentées de manière disproportionnées dans les nouvelles entreprises de services. Au cours des dix dernières années, l'écart de proportion de travailleurs indépendants entre hommes et femmes s'est comblé dans la quasi-totalité des pays. Des disparités importantes subsistent néanmoins entre les sexes : dans les pays de l'OCDE, une femme sur dix ayant un emploi est une travailleuse indépendante, soit une proportion presque inférieure de moitié à celle des hommes (17\% des actifs occupés de sexe masculin étant des travailleurs indépendants).

\section{Les écarts de salaire dans le secteur manufacturier se creusent dans de nombreux pays}

Dans un grand nombre de pays, la croissance de la productivité du travail après la crise dans les PME du secteur manufacturier a été plus lente que dans les grandes entreprises, ce qui a accentué les écarts de productivité antérieurs, en particulier en Allemagne, en Belgique, en Lettonie, en République slovaque et en République tchèque. De même, les écarts de salaire entre grandes et petites entreprises manufacturières se sont creusés dans tous les pays d'Europe de l'Est appartenant à l'OCDE - sauf en Pologne -, dans les États baltes, en Norvège et au Royaume-Uni entre 2008 et 2014.

\section{Les outils numériques ont ouvert de nouvelles voies et de nouveaux marchés aux microentrepreneurs}

Le développement d'outils et de plateformes numériques abordables a offert aux microentreprises de nouvelles possibilités d'accéder aux marchés étrangers d'une façon qui aurait été précédemment inimaginable. De nouvelles données de l'enquête sur l'avenir des entreprises (Future of Business Survey), réalisée conjointement chaque mois par Facebook, l'OCDE et la Banque mondiale auprès de PME présentes sur internet, montrent que même les travailleurs pour leur propre compte (c'est-à-dire les travailleurs indépendants sans salarié) peuvent réaliser une part importante de leurs activités à l'exportation, en capitalisant sur les outils numériques, malgré la petite taille de leur entreprise. Deux entreprises exportatrices sur trois ont répondu que plus de la moitié de leur chiffre d'affaires international dépendait d'outils en ligne, et près de la moitié ( $45 \%$ ) ont indiqué que plus de $75 \%$ de leur chiffre d'affaires international étaient tributaires d'outils en ligne.

Les données plus récentes de l'enquête sur l'avenir des entreprises confirment également les conclusions antérieures selon lesquelles les entreprises présentes à l'international sont plus confiantes quant à leur situation actuelle et à leurs perspectives futures d'activité, et qu'elles sont également plus susceptibles d'avoir des perspectives positives en matière de création d'emplois.

Cela vaut également pour les travailleurs pour leur propre compte qui sont présents à l'international. Cela dit, l'enquête révèle aussi que les grandes entreprises ont généralement une évaluation plus positive de leur situation que les petites entreprises. Cela tient à l'ampleur des difficultés auxquelles sont confrontées les microentreprises dans le cadre de la réalisation et du développement de leurs activités, qu'il s'agisse de se conformer à la réglementation, d'obtenir des financements, de recruter et de retenir des salariés qualifiés, ou encore de trouver des partenaires commerciaux. 


\section{L'émergence des " travailleurs à la demande "}

Une tendance générale observée dans la plupart des pays réside dans l'augmentation du nombre de travailleurs indépendants ayant seulement une activité à temps partiel. Le travail indépendant à temps partiel a connu une progression considérable au cours des dix dernières années, ce qui tient en partie aux nouvelles possibilités offertes par l'émergence de l'" économie à la demande ", c'est-à-dire par l'essor de formes d'emploi flexibles, qui complètent les emplois à temps plein ou s'y substituent. Si le travail à la tâche n'a en soi rien de nouveau (l'industrie du spectacle, par exemple, y a toujours eu recours), il est aujourd'hui offert et demandé par un groupe de personnes plus vaste et plus divers et couvre un éventail de services plus large que jamais auparavant.

L'émergence des travailleurs à la demande soulève de nouvelles questions sur l'opportunité d'utiliser la proportion de travailleurs indépendants ou leur nombre comme variable indicatrice de l'ampleur de l'entrepreneuriat, la relation entre économie à la demande et activité entrepreneuriale n'étant aucunement évidente. Les acteurs de l'économie à la demande peuvent, certes, être de petits entrepreneurs, mais nombre des travailleurs à la demande fournissent leurs services dans des conditions qui présentent de fortes similitudes avec la situation des salariés classiques, notamment en l'absence de risque entrepreneurial. Par ailleurs, la flexibilité associée au travail à la tâche contraste souvent avec l'emploi salarié classique, et peut encourager les entrepreneurs en herbe à réaliser leur projet de nouvelles activités tout en restant en mesure de subvenir à leurs besoins.

L'évaluation de la contribution à l'entrepreneuriat des travailleurs à la demande pose donc des problèmes de mesure, certains éléments laissant à penser que l'économie à la demande peut parfois réduire l'activité entrepreneuriale, en particulier lorsque les plateformes de services à la demande se substituent à un entrepreneuriat de qualité médiocre, au lieu de compléter un entrepreneuriat de haute qualité.

Nous nous attacherons à remédier à ces problèmes de mesure et à étudier les possibilités d'améliorer la disponibilité des données dans ce domaine dans le cadre de cette publication, qui vise de manière générale à offrir de meilleures données pour la mesure de l'entrepreneuriat. Les autres efforts déployés à cet égard consistent notamment à distinguer les nouvelles entreprises en fonction de leur contrôle capitalistique, de leur présence à l'international et de leur intégration dans les chaînes de valeur mondiales, à élaborer de meilleures données sur les disparités entre hommes et femmes et aussi, de manière plus générale, à mettre en lumière les écueils à éviter et les réserves à prendre en compte sur le plan statistique pour l'utilisation des données sur l'entrepreneuriat. 



\section{Guide de Lecture}

L

La présente publication détaille les indicateurs de l'entrepreneuriat recueillis par le Programme d'indicateurs de l'entrepreneuriat OCDE-Eurostat (PIE). Lancé en 2006, ce programme élabore différentes mesures de l'entrepreneuriat et de ses déterminants selon un cadre conceptuel qui fait la distinction entre la manifestation de l'entrepreneuriat, les facteurs qui l'influencent et ses impacts sur l'économie. Une caractéristique de ce programme est qu'il ne fournit pas de mesure composite unique de l'entrepreneuriat global au sein d'une économie. Au contraire, il tient compte des multiples facettes de celui-ci et s'articule autour d'une suite d'indicateurs de la performance entrepreneuriale qui reflètent chacun un ou plusieurs de ces aspects. Le plus important est sans doute la prise en compte, dans ce programme, du fait que l'entrepreneuriat n'est pas seulement une question d'entreprises de création récente (start-ups) ou de nombre de travailleurs indépendants : les entrepreneurs et les forces entrepreneuriales peuvent se rencontrer dans de nombreuses entreprises existantes, et il est tout aussi important de comprendre le dynamisme que ces acteurs peuvent insuffler à l'économie que d'appréhender la dynamique des entreprises de création récente ou le travail indépendant.

Les indicateurs de la performance entrepreneuriale, calculés par les offices statistiques nationaux, sont présentés pour les pays suivants : Allemagne, Australie, Autriche, Belgique, Brésil, Canada, Chili, Colombie, Corée, Danemark, Espagne, Estonie, États-Unis, Fédération de Russie, Finlande, France, Hongrie, Israël, Italie, Japon, Lettonie, Lituanie, Luxembourg, Mexique, Norvège, Nouvelle-Zélande, Pays-Bas, Portugal, République slovaque, République tchèque, Roumanie, Royaume-Uni, Slovénie, Suède et Suisse.

Pour chacun de ces indicateurs, un petit texte explique ce qu'il mesure, comment il est défini et quel est son intérêt pratique. Des précisions sur la comparabilité de l'indicateur d'un pays à un autre sont également fournies.

Cette publication contient également des données sur le " capital-risque », facteur clé de l'entrepreneuriat, ainsi qu'une liste, disponible en annexe, d'indicateurs de déterminants de l'entrepreneuriat conjointement avec leurs sources.

\section{Indicateurs}

Les indicateurs du PIE n'ont pas tous le même degré de sophistication. Certains font depuis longtemps partie de collectes de données régulières, tandis que d'autres ne sont en place que dans un nombre restreint de pays et n'ont pas encore de définition harmonisée, les échanges et les travaux pour l'établir étant toujours en cours. Les indicateurs présentés dans ces pages reflètent cette diversité :
A. Créations d'entreprises
B. Faillites
C. Travail indépendant
D. Perspectives économiques et perspectives de créations d'emplois 
E. Entreprises par classe de taille

F. Emploi par taille d'entreprise

G. Valeur ajoutée par taille d'entreprise

H. Chiffre d'affaires par taille d'entreprise

I. Rémunération des salariés par taille d'entreprise

J. Productivité de la main-d'œuvre par taille d'entreprise

K. Taux de naissance des entreprises

L. Taux de décès des entreprises

M. Survie des entreprises

N. Créations et destructions d'emplois dues aux naissances et décès d'entreprises

O. Taux d'entreprises à forte croissance

P. Concentration des échanges

Q. Échanges par taille d'entreprise

R. Proximité du marché

S. Échanges par type de contrôle capitalistique des entreprises

T. Travail indépendant: différences hommes-femmes

U. Travail indépendant chez les jeunes

V. Revenus du travail indépendant

W. Attitude à l'égard de l'entrepreneuriat

$\mathrm{X}$. Investissements de capital-risque

Les indicateurs A et B proviennent de la Base de données de l'OCDE concernant les indicateurs actualisés de l'entrepreneuriat, dont les sources sont énoncées à l'annexe A. La source de l'indicateur $\mathrm{C}$ est la base de données des Principaux indicateurs économiques (PIE) de l'OCDE. Quant à l'indicateur D, il est tiré des résultats de l'enquête sur l'avenir des entreprises (Future of business survey), conçue par Facebook en collaboration avec la Direction des statistiques de l'OCDE et la Banque mondiale.

Pour les indicateurs E à $\mathrm{O}$, la source est la Base de données de l'OCDE sur les statistiques structurelles et démographiques des entreprises (SDBS). Les indicateurs E à J font référence aux statistiques structurelles sur les entreprises, tandis que les indicateurs L à P proviennent des statistiques démographiques des entreprises, généralement calculées à partir des répertoires d'entreprises. Les indicateurs P à S proviennent de la Base de données TEC (statistiques du commerce en fonction des caractéristiques des entreprises) de l'OCDE. Les données SDBS et TEC sont recueillies chaque année à l'aide de questionnaires harmonisés soumis aux offices statistiques nationaux.

Les indicateurs du travail indépendant proviennent des enquêtes sur la population active et des données de recensement (indicateurs $\mathrm{T}$ et $\mathrm{U}$ ), ainsi que des enquêtes sur les revenus (indicateur V).

L'indicateur W est tiré de l'enquête sur l'avenir des entreprises (Future of Business Survey).

L'indicateur X provient de la Base de données de l'OCDE sur le financement de l'entrepreneuriat.

\section{Ventilation par classe de taille}

En règle générale, les indicateurs des statistiques structurelles sur les entreprises distinguent cinq classes de taille - en fonction du nombre de personnes occupées - en vertu 
desquelles les données des pays et les variables peuvent la plupart du temps être regroupées comme suit : 1-9, 10-19, 20-49, 50-249, 250+. Toutefois, toutes les informations nationales ne correspondent pas parfaitement à cette taxinomie, et les disparités éventuelles sont indiquées dans chaque chapitre.

S'agissant des données démographiques sur les entreprises, la grande majorité des entreprises de création récente étant des microentreprises, la ventilation habituelle des données collectées est la suivante : 1-4, 5-9, 10+ salariés.

Pour les données du commerce par caractéristique d'entreprise (CCE), la ventilation par classe de taille est la suivante : 0-9, 10-49, 50-249, 250+ salariés ; par ailleurs, une classe intitulée "Indéterminé » contient des informations sur les échanges pour les entreprises dont la taille n'a pu être établie.

Dans cette publication, les microentreprises ont entre 1 et 9 salariés, les petites entreprises entre 10 et 49 salariés, les moyennes entreprises entre 50 et 249 salariés, et les grandes entreprises 250 salariés et plus. La catégorie des " petites et moyennes entreprises " (PME) correspond à la classe de taille 1-249 salariés. Sur les graphiques établis à partir de la base de données TEC, les PME ont une taille de 0-249 salariés.

\section{Ventilation par activité}

Les données sont présentées selon la Classification internationale type, par industrie, de toutes les branches d'activité économique, révision 4 (CITI rév. 4). L'ensemble de l'activité du secteur marchand recouvre : les activités extractives (05-09), les activités de fabrication (10-33), la production d'électricité, de gaz, de vapeur et la climatisation (35), la distribution d'eau, le réseau d'assainissement, la gestion des déchets et les activités de remise en état (36-39), la construction (41-43) et les services (45-82). Les services regroupent : le commerce de gros et de détail et les réparations de véhicules automobiles et de motocycles (45-47), le transport et l'entreposage (49-53), les activités d'hébergement et de restauration (55-56), l'information et la communication (58-63), les activités financières et d'assurance (64-66), les activités immobilières (68), les activités professionnelles, scientifiques et techniques (69-75), ainsi que les activités de services administratifs et d'appui (77-82).

Concernant les statistiques structurelles des entreprises (chapitres 2 et 3), toute la section des activités financières et d'assurance (64-68) est exclue des services, sauf pour le Canada et la Corée ; concernant la démographie des entreprises (chapitres 4 et 5), les activités des sociétés de portefeuille (642) sont exclues des activités financières et d'assurance, sauf pour la Corée, les États-Unis, Israël et le Mexique.

Dans les chapitres 4 à 6 , il est fait référence à l'agrégat Industrie, qui comprend les secteurs 05 à 39 . Au chapitre 6, l'économie totale englobe tous les secteurs de la CITI rév. 4, de 01 to 99 (c'est-à-dire des activités agricoles aux activités des organisations extraterritoriales).

Pour certains pays, les données fournies par les offices statistiques nationaux suivent le système national de classification. Dans cette publication, ces données ont été converties au système de la CITI rév. 4 pour les pays suivants. Les données du Canada et du Mexique sont répertoriées selon le système de classification de l'industrie d'Amérique du Nord pour 2012, à deux chiffres ou plus. Concernant le Japon, les données structurelles de 2013 sur le nombre d'entreprises et le nombre de salariés proviennent de l'édition 2014 du recensement économique des entreprises (2014 Economic Census for Business Frame) et sont présentées selon la Classification industrielle standard japonaise rév. 13, à deux chiffres ou plus. Pour la Corée, les données structurelles 2006-14 sur le nombre d'entreprises et le nombre de salariés proviennent du recensement des établissements qui, de même que les données sur 
la démographie des entreprises, sont compilées selon la Classification industrielle standard coréenne, à deux chiffres ou plus. Les États membres de l'Union européenne, la Norvège, la Suisse et la Turquie ont adopté quant à eux la nomenclature statistique des activités économiques dans la Communauté européenne (NACE) rév. 2, à trois chiffres ou plus.

Les données sur la démographie des entreprises des États-Unis et les données structurelles sur les entreprises de la Fédération de Russie sont compilées conformément à la CITI rév. 3.

Les données fournies par les offices statistiques nationaux des autres pays sont présentées au format de la CITI rév. 4.

\section{Codes pays}

Les chiffres cités dans la présente publication utilisent les codes ISO (ISO3) des noms de pays, selon le tableau ci-dessous :

\begin{tabular}{llll}
\hline ARG & Argentine & LVA & Lettonie \\
AUS & Australie & LTU & Lituanie \\
AUT & Autriche & LUX & Luxembourg \\
BEL & Belgique & MEX & Mexique \\
BRA & Brésil & NLD & Pays-Bas \\
CAN & Canada & NZL & Nouvelle-Zélande \\
CHL & Chili & NOR & Norvège \\
COL & Colombie & PER & Peru \\
CZE & République tchèque & PRT & Portugal \\
DNK & Danemark & ROU & Roumanie \\
EST & Estonie & RUS & Fédération de Russie \\
FIN & Finlande & SVK & République slovaque \\
FRA & France & SVN & Slovénie \\
HUN & Hongrie & ESP & Espagne \\
DEU & Allemagne & ZAF & Afrique du Sud \\
IND & Inde & SWE & Suède \\
IDN & Indonésie & CHE & Suisse \\
ISR & Israell & TUR & Turquie \\
ITA & Italie & GBR & Royaume-Uni \\
JPN & Japon & USA & États-Unis \\
KOR & Corée & VNM & Viêt Nam \\
\hline
\end{tabular}

\section{Cadre du PIE}

Le PIE définit l'entrepreneuriat comme le phénomène associé à l'activité entrepreneuriale, action humaine consistant à entreprendre pour générer de la valeur en créant ou en développant des activités économiques grâce à la découverte et à l'exploitation de nouveaux produits, procédés ou marchés. En ce sens, l'entrepreneuriat est un phénomène qui se manifeste dans l'ensemble de l'économie sous de nombreuses formes différentes et produit de nombreux résultats eux aussi différents. Ces derniers ne sont pas toujours liés à la création de richesses financières et peuvent, par exemple, concerner l'amélioration de l'emploi, la lutte contre les inégalités ou la lutte contre les problèmes environnementaux. La mission du PIE consiste à permettre de mieux appréhender ces manifestations multiples de l'entrepreneuriat. Le fait étant établi qu'aucun indicateur ne peut à lui seul embrasser fidèlement l'entrepreneuriat, il a été élaboré un ensemble de mesures qui traduisent tel 
ou tel aspect entrepreneurial ou type d'entrepreneuriat ; ces mesures sont appelées les indicateurs PIE de la performance entrepreneuriale. Il en existe actuellement une vingtaine.

Le PIE tente par ailleurs de mesurer tous les aspects de l'entrepreneuriat en s'intéressant non seulement à la manifestation du phénomène entrepreneurial, mais aussi aux facteurs qui l'influencent. Ces facteurs vont de la situation du marché et du cadre réglementaire à la culture et aux conditions d'accès aux financements. Si certains types de déterminants de l'entrepreneuriat se prêtent plus aisément à ce travail de mesure (par exemple, l'existence et la rigueur d'un droit de la concurrence, ou les coûts administratifs engendrés par la création d'une entreprise dans un pays), la difficulté consiste pour d'autres à trouver des objets mesurables (par exemple le tutorat-investissement, c'est-à-dire les capitaux des business angels) ou à appréhender la nature exacte de leurs liens avec l'entrepreneuriat (d'un point de vue par exemple culturel). À cet égard, le PIE est investi d'une mission importante, qui consiste à participer aux travaux et à faire progresser la recherche sur les déterminants de l'entrepreneuriat qui sont moins bien compris et plus difficilement mesurables. L'annexe B présente une liste complète d'indicateurs des déterminants avec les sources de données correspondantes.

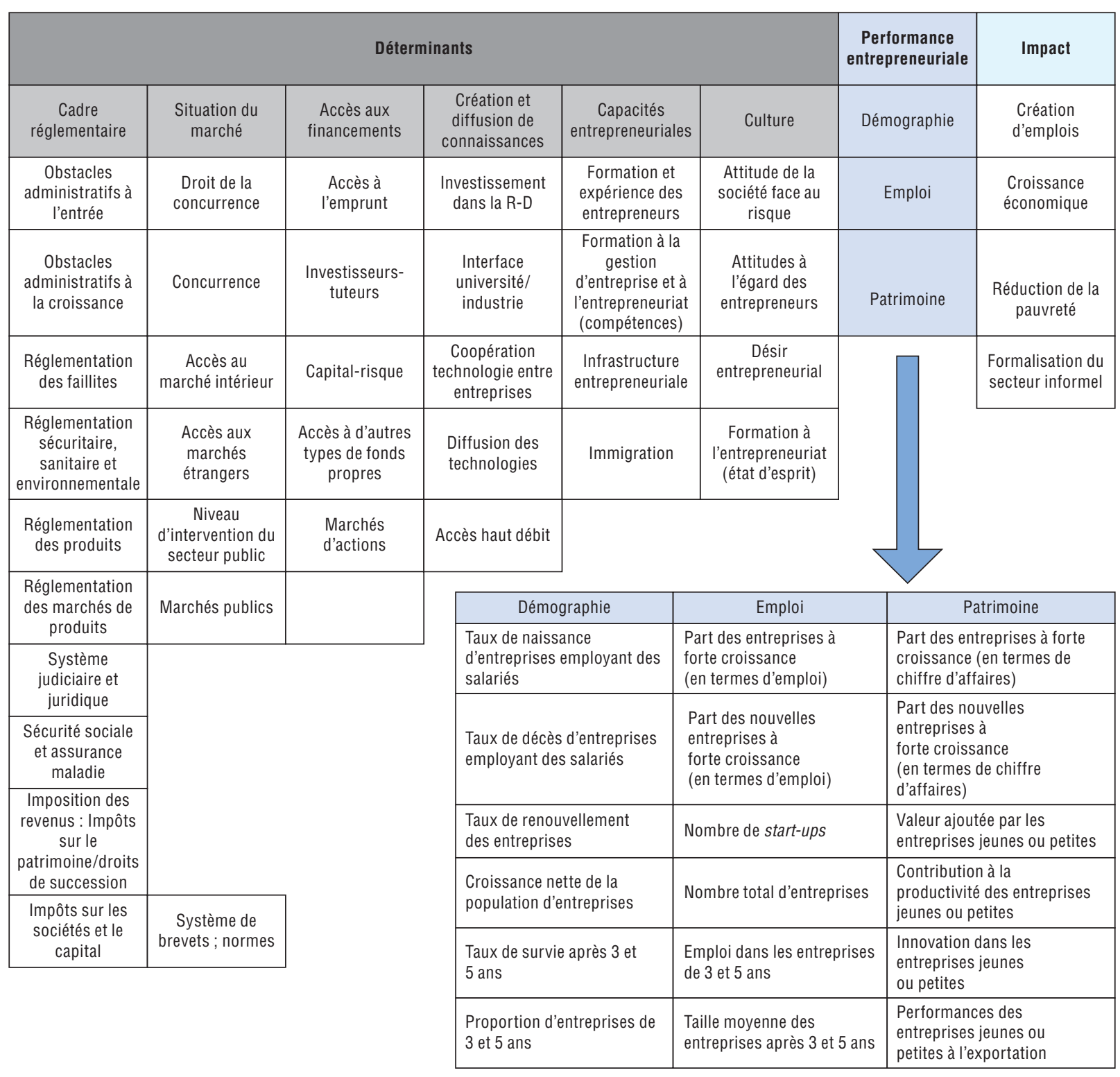





\section{Chapitre 1}

\section{Évolutions récentes de l'entrepreuneuriat}

Les indicateurs de court terme présentés dans ce chapitre fournissent des informations récentes sur la dynamique du secteur des entreprises et le travail indépendant. Ils fournissent un aperçu actualisé de la situation de l'entrepreneuriat, qui permet d'en savoir plus sur les perspectives en matière de croissance et d'emploi dans la zone OCDE. 


\section{Rebond des créations d'entreprises dans la plupart des pays}

Dans la plupart des pays de l'OCDE où des données sont disponibles, le nombre de créations d'entreprises continue de se redresser et dépasse, dans de nombreux cas, les sommets atteints avant la crise. Parmi les économies de l'OCDE pour lesquelles on dispose de données actualisées, neuf ont vu les créations d'entreprises s'inscrire en hausse récemment (jusqu'au premier trimestre 2017) : Australie, Belgique, États-Unis, France, Hongrie, Islande, Norvège, Pays-Bas et Suède (graphique 1.1).

Par ailleurs, la poursuite de l'orientation à la baisse du nombre de créations d'entreprises dans certains pays ces dernières années peut masquer d'autres évolutions. En Italie et en Allemagne, par exemple, ce déclin reflète un recul du nombre d'entreprises individuelles, alors que le nombre de nouvelles entreprises prenant d'autres formes juridiques a augmenté (graphique 1.2).

Les hausses enregistrées ces dernières années semblent avoir été essentiellement tirées par le secteur des services. En Allemagne, au Canada, aux États-Unis et en France, la croissance tendancielle des créations d'entreprises dans ce secteur a dépassé celle du secteur manufacturier (graphique 1.3). 


\section{Graphique 1.1. Créations d'entreprises}

Tendance-cycle, $2012=100$
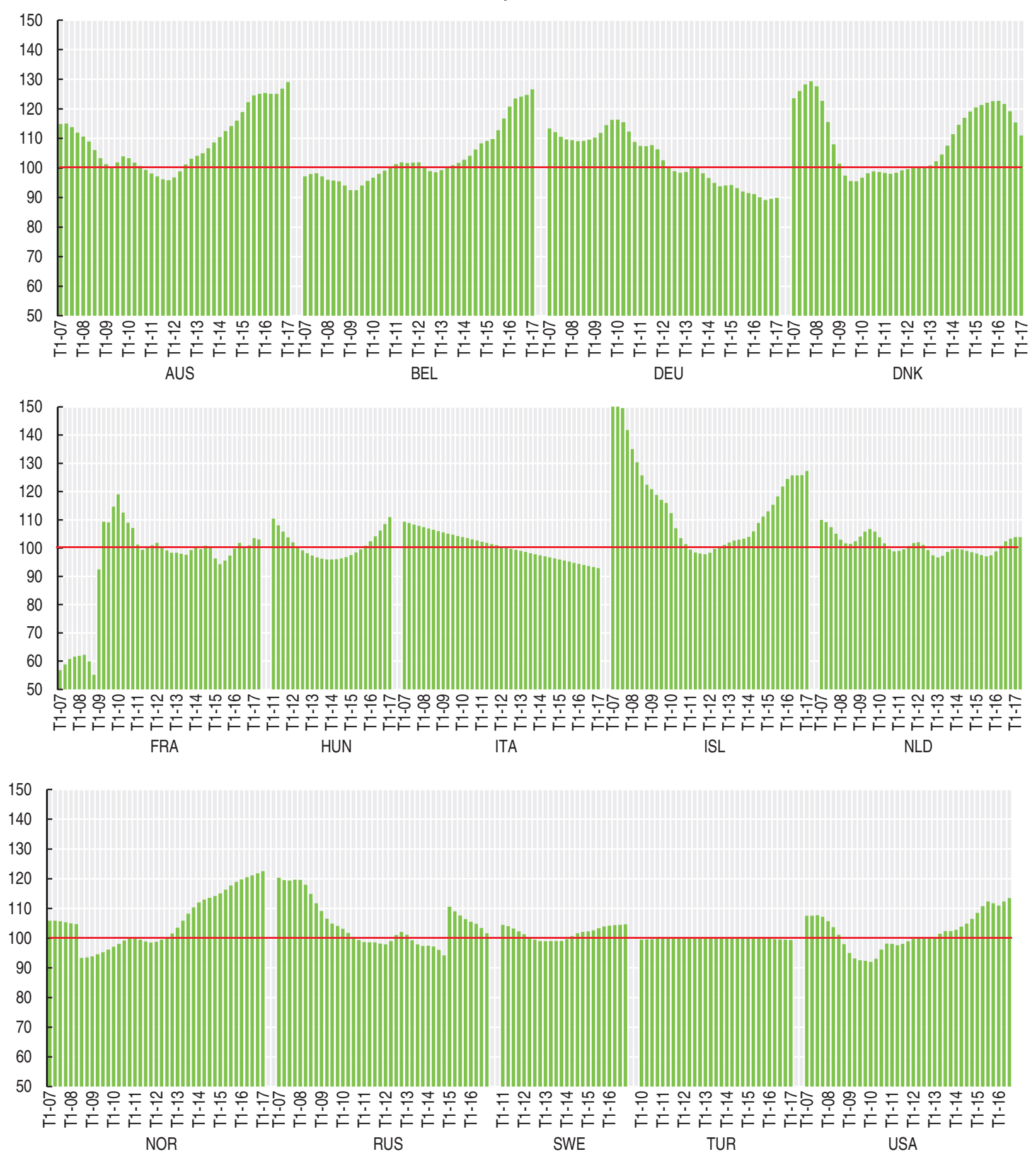

Source : Base de données de l'OCDE concernant les indicateurs actualisés de l'entrepreneuriat, juillet 2017. 


\section{Graphique 1.2. Créations d'entreprises par forme juridique}

Tendance-cycle, $2012=100$
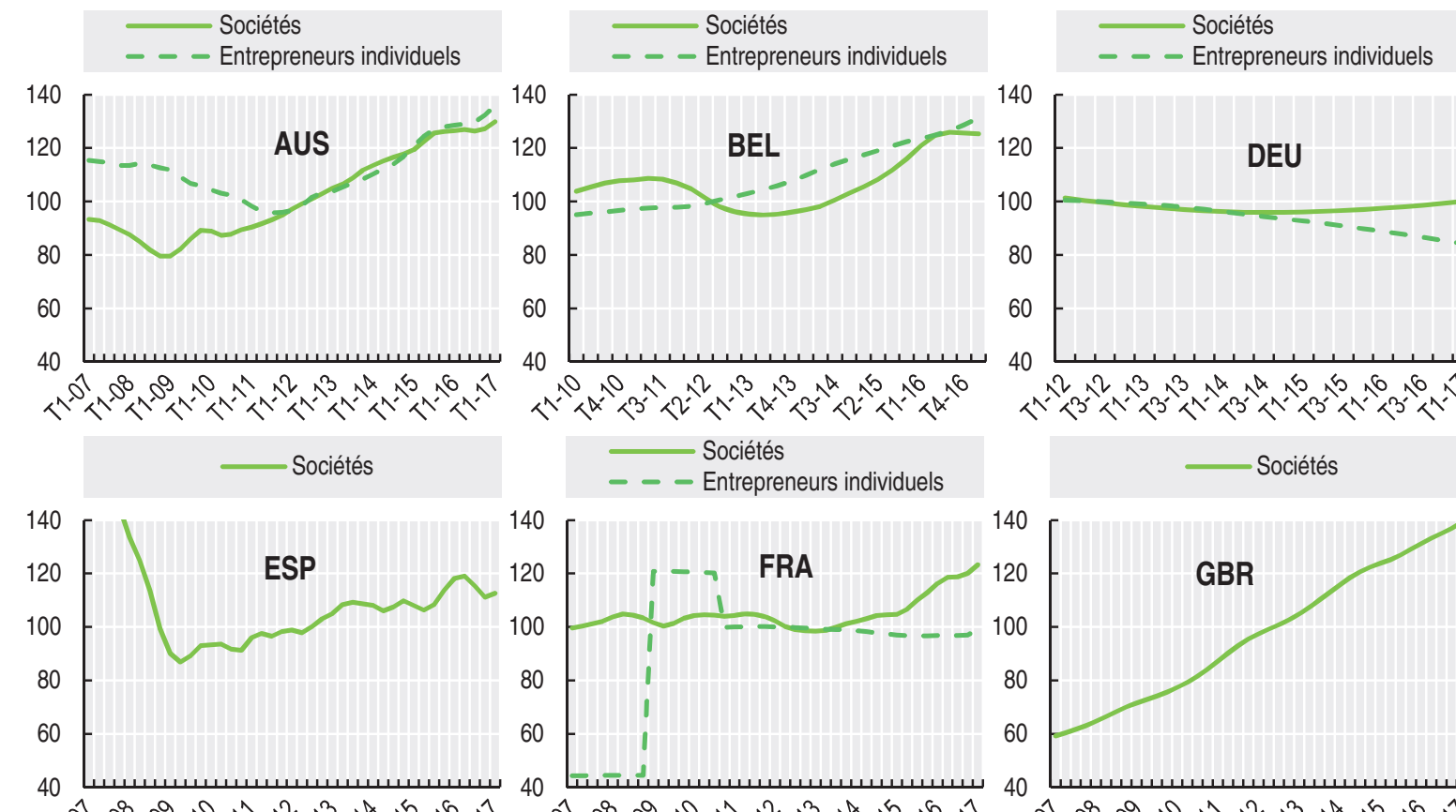

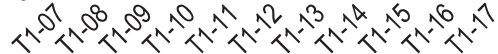

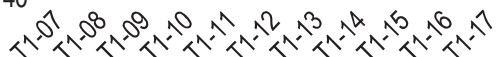

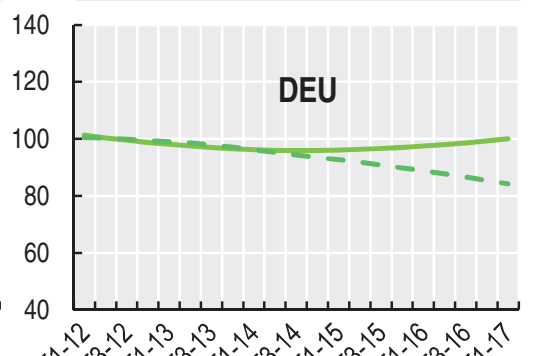

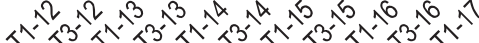
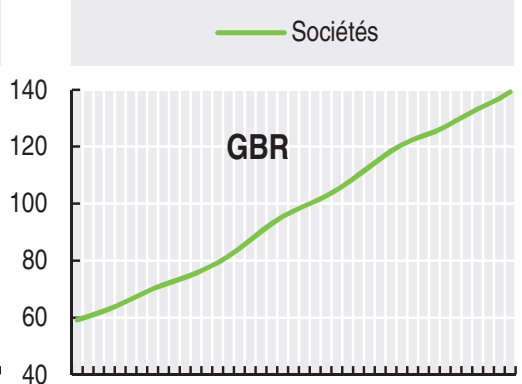

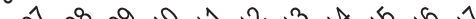
_ Sociétés

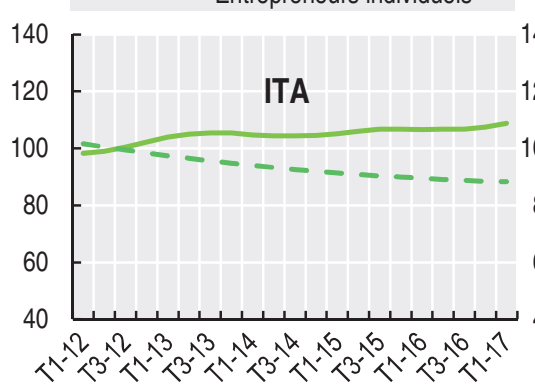

Sociétés

- - Entrepreneurs individuels
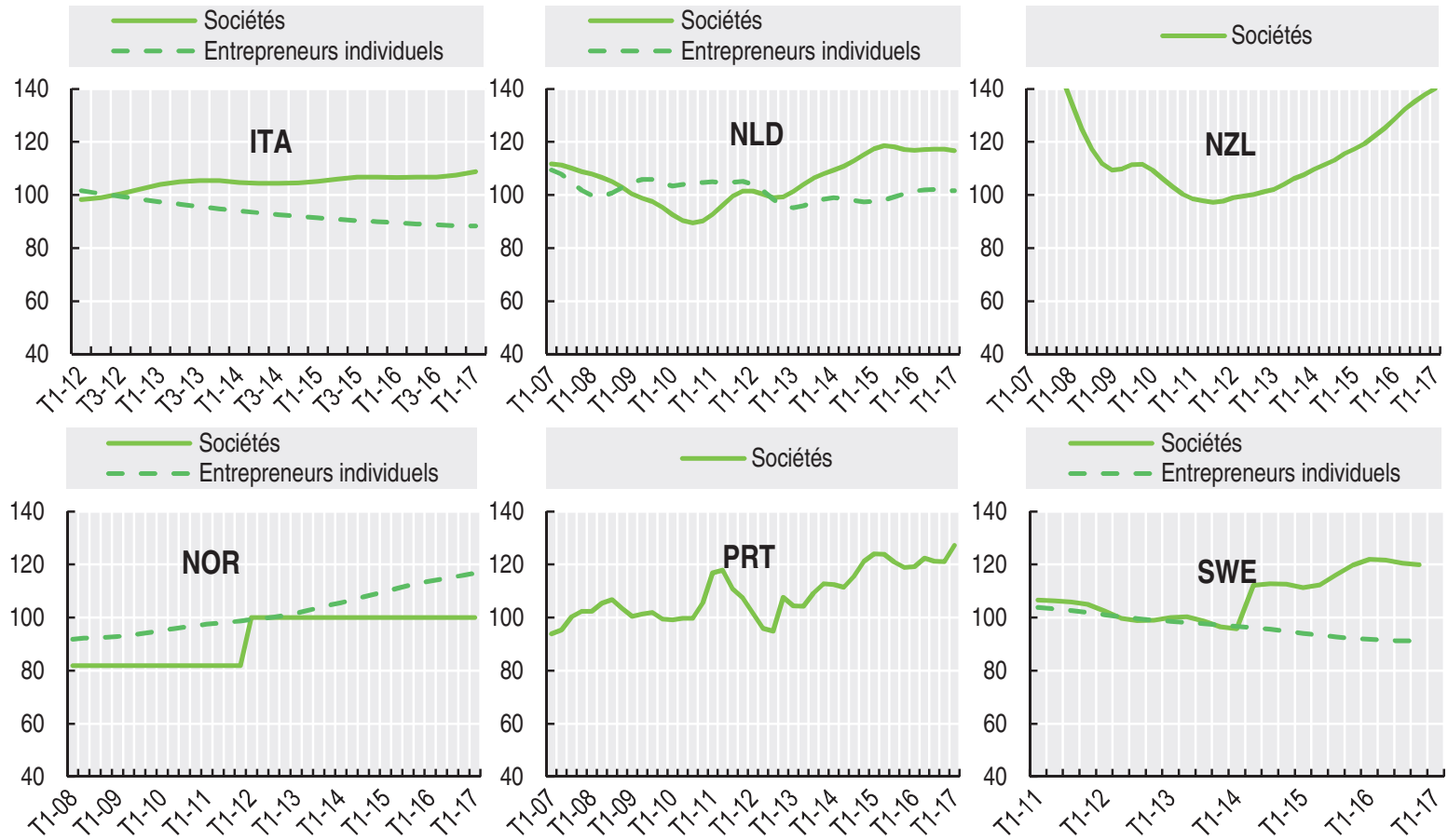

Source : Base de données de l'OCDE concernant les indicateurs actualisés de l'entrepreneuriat, juillet 2017. 


\section{Graphique 1.3. Créations d'entreprises par grand secteur d'activité, échantillon de pays}

Tendance-cycle, $2012=100$

Canada

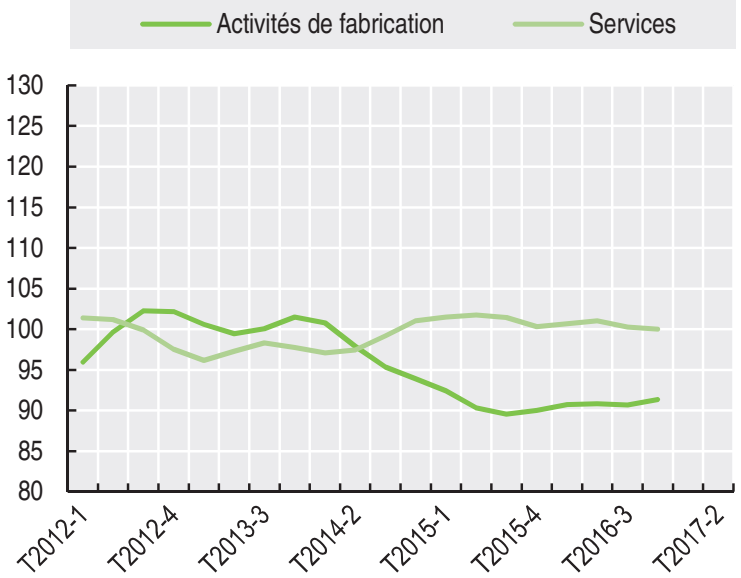

Allemagne
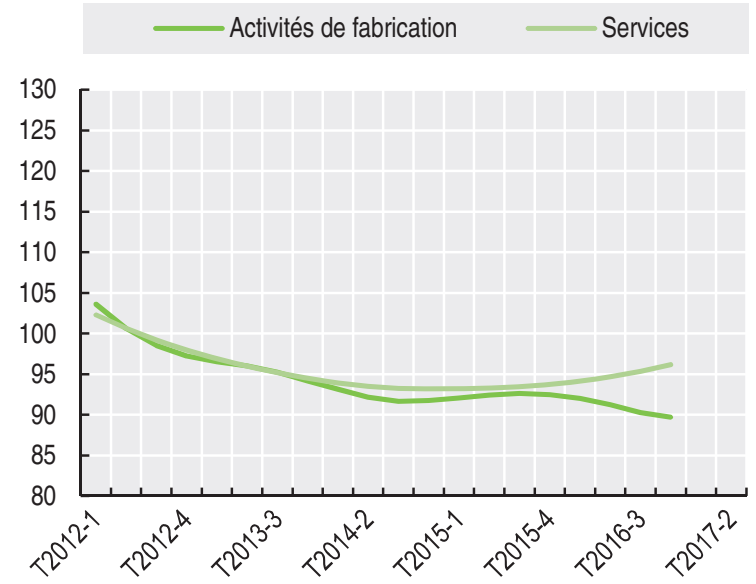

Norvège
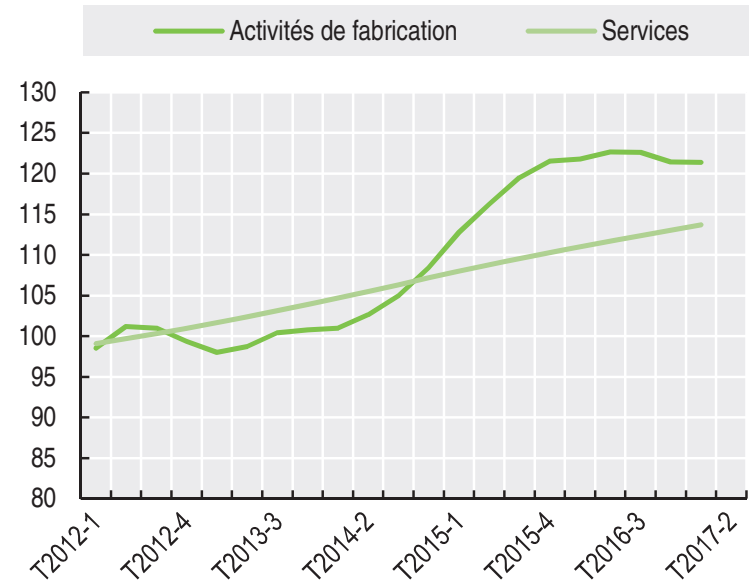

France

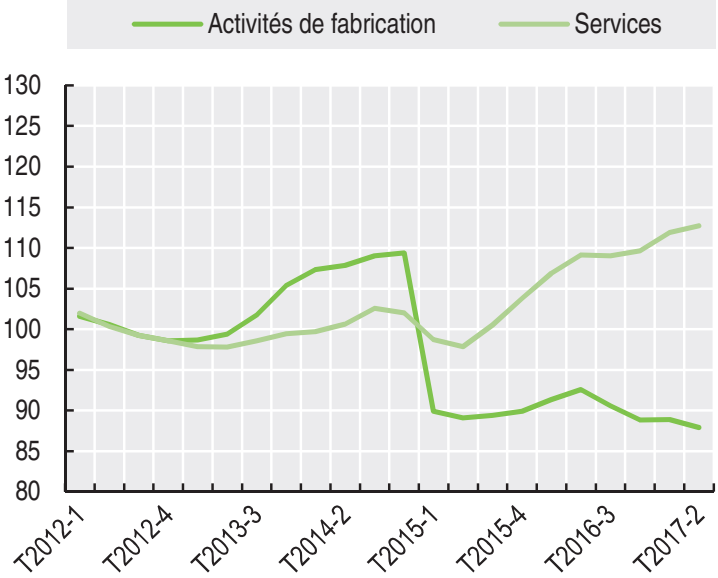

Pays-Bas
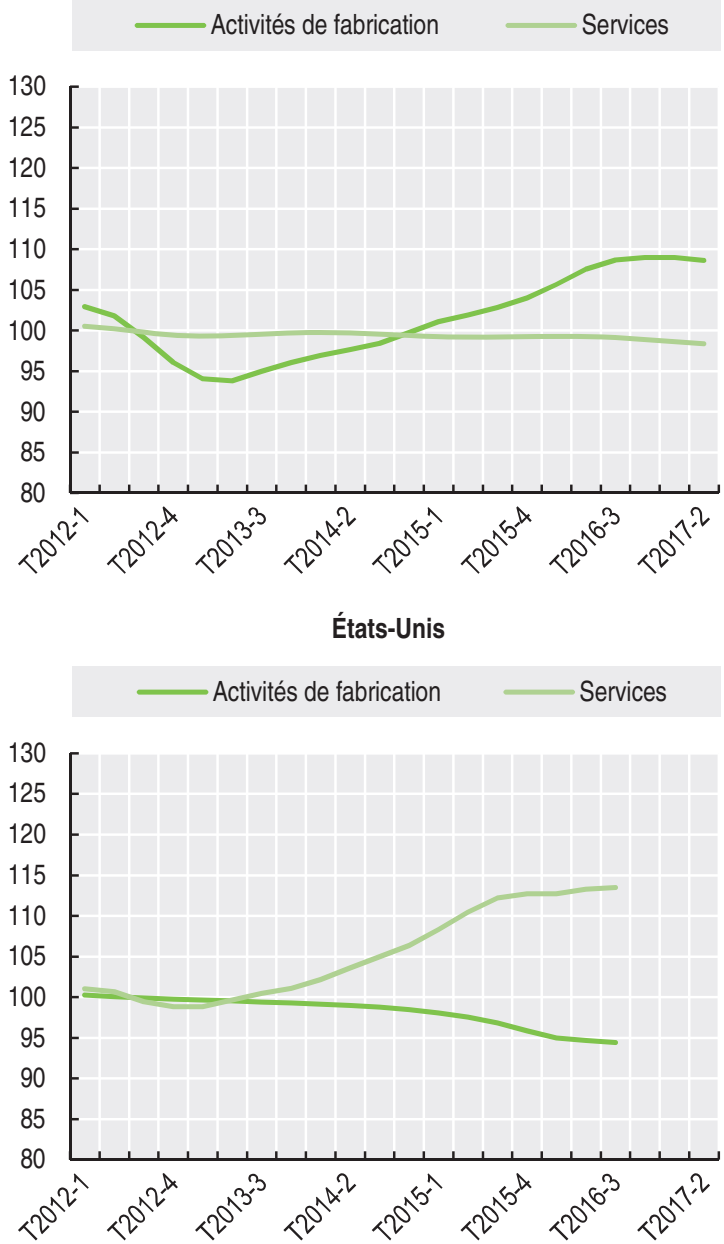

Source : Base de données de l'OCDE concernant les indicateurs actualisés de l'entrepreneuriat, juillet 2017. 


\section{Encadré 1.1. Gros plan sur la baisse séculaire des taux de créations d'entreprises}

Dans un contexte d'évolution à la baisse de la productivité, le phénomène désormais qualifié de « baisse séculaire " des taux de créations d'entreprises fait l'objet depuis quelques années de vastes débats. L'attention est essentiellement portée sur les États-Unis, pour lesquels on dispose de séries chronologiques relativement longues remontant jusqu'aux années 80 (Decker et al., 2016, Haltiwanger 2016). Des études comparables, quoique fondées sur des séries chronologiques nettement plus courtes (Blanchenay et al., à paraitre) aboutissent à des conclusions similaires pour d'autres pays.

Les données actualisées présentées dans cette publication laissent à penser que le mouvement de baisse séculaire serait en train de s'infléchir, bien qu'il soit encore trop tôt pour l'affirmer avec certitude. Afin de donner plus de poids à cette (tentative d') hypothèse, il est utile de replacer les débats autour des créations d'entreprises dans leur contexte, ou tout au moins de mettre en évidence le caractère statistique de la construction de ces données et la manière dont elles doivent être interprétées dans les analyses.

Bien souvent dans les analyses, les taux de créations figurant dans les statistiques sur les entreprises sont considérés de façon analogue aux taux de naissance calculés pour la population humaine générale, même si leur application diffère. Toutefois, on oublie souvent dans ces débats que, contrairement à la population humaine, les entreprises existantes ne donnent généralement pas naissance à de nouvelles entrées et ne créent pas de nouvelles entreprises, et lorsque c'est le cas, les entreprises ainsi créées sont souvent interprétées en termes de " croissance » des entreprises existantes et non comme des créations.

Il est implicite dans cette analogie que le stock d'entreprises existant est considéré comme un indicateur du vivier d'entrepreneuriat à partir duquel peuvent émerger de nouvelles entreprises, d'où leur utilisation au dénominateur dans le calcul des taux de créations d'entreprises. Cette méthode s'est avérée, et continue de s'avérer, un indicateur très utile de l'entrepreneuriat au sein d'une économie donnée, ce qui explique la large place qui lui est accordée dans cette publication. Toutefois, il convient d'émettre des réserves d'ordre statistique qui peuvent avoir une incidence sur la comparabilité des données dans le temps et entre pays.

Deux pays, par exemple, qui, pour une année donnée, affichent une population générale et un nombre de créations d'entreprises exactement identiques, peuvent avoir des taux très différents de créations d'entreprises si leur population d'entreprises diffère. En effet, si l'un d'entre eux est une économie émergente et l'autre une économie développée parvenue à maturité, on peut penser que, par un phénomène de rattrapage économique, le nombre de créations d'entreprises dans l'économie émergente sera plus important que dans l'économie développée. L'application d'un chiffre plus petit au dénominateur (nombre d'entreprises) accentuerait alors cet écart. Ce n'est peut-être pas un hasard si les taux de création d'entreprises présentés dans ce rapport sont plus élevés pour les anciennes économies en transition et les économies émergentes que pour les économies développées plus matures.

Dans ce contexte, il est utile de garder un œil sur les niveaux, et pas uniquement sur les taux, de créations d'entreprises. Le graphique 1.4 ci-après, par exemple, montre l'évolution du nombre d'établissements employant des salariés aux États-Unis au cours du dernier quart de siècle, et révèle une tendance nettement à la hausse, malgré le creux de la crise, ce qui vient légèrement nuancer la thèse de la baisse séculaire. 


\section{Encadré 1.1. Gros plan sur la baisse séculaire des taux de créations d'entreprises (suite)}

Graphique 1.4. Nombre d'établissements employant des salariés aux États-Unis En millions

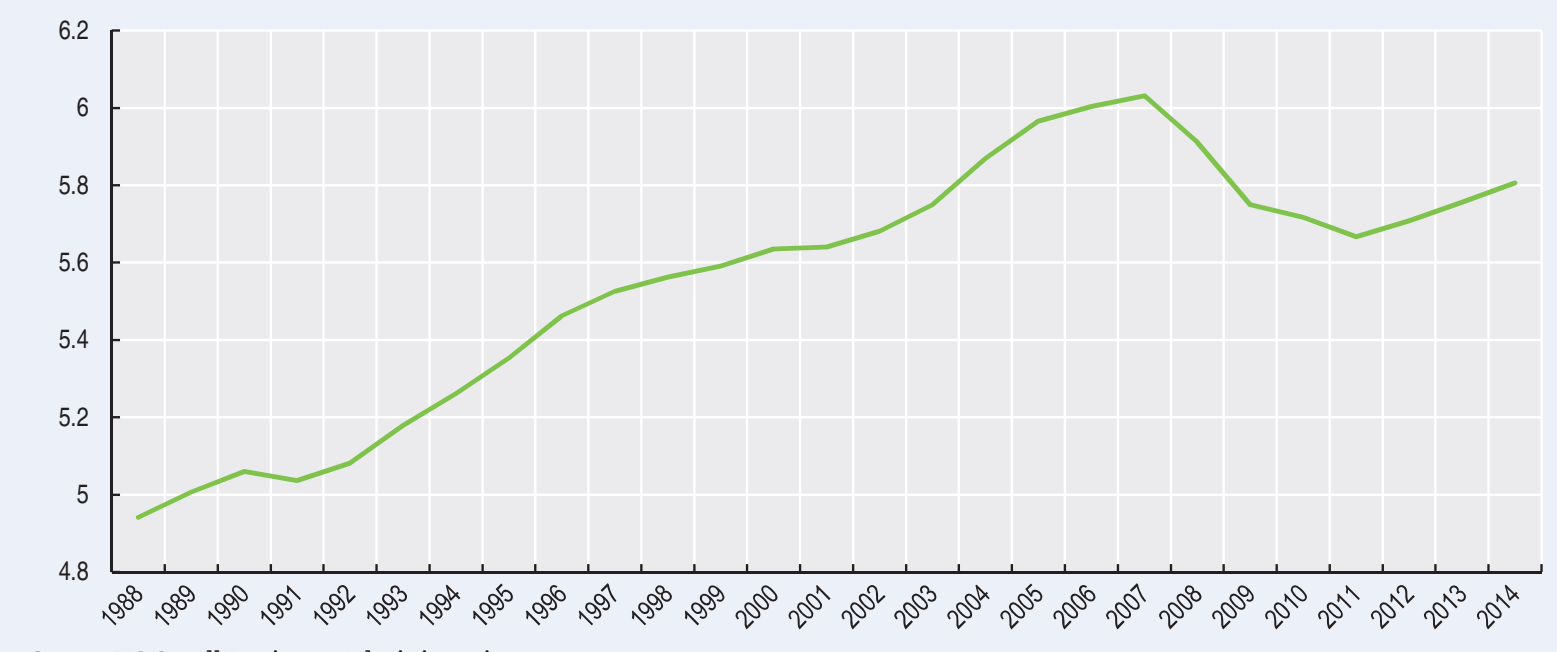

Source: US Small Business Administration.

L'examen de la forte croissance de la population de grands établissements (plus de 500 salariés) permet également de replacer la thèse de la baisse séculaire dans un contexte plus large, la concentration accrue du marché ayant pu évincer de nouveaux entrants potentiels.

\section{Graphique 1.5. Établissements employant plus de 500 salariés aux États-Unis}

En nombre

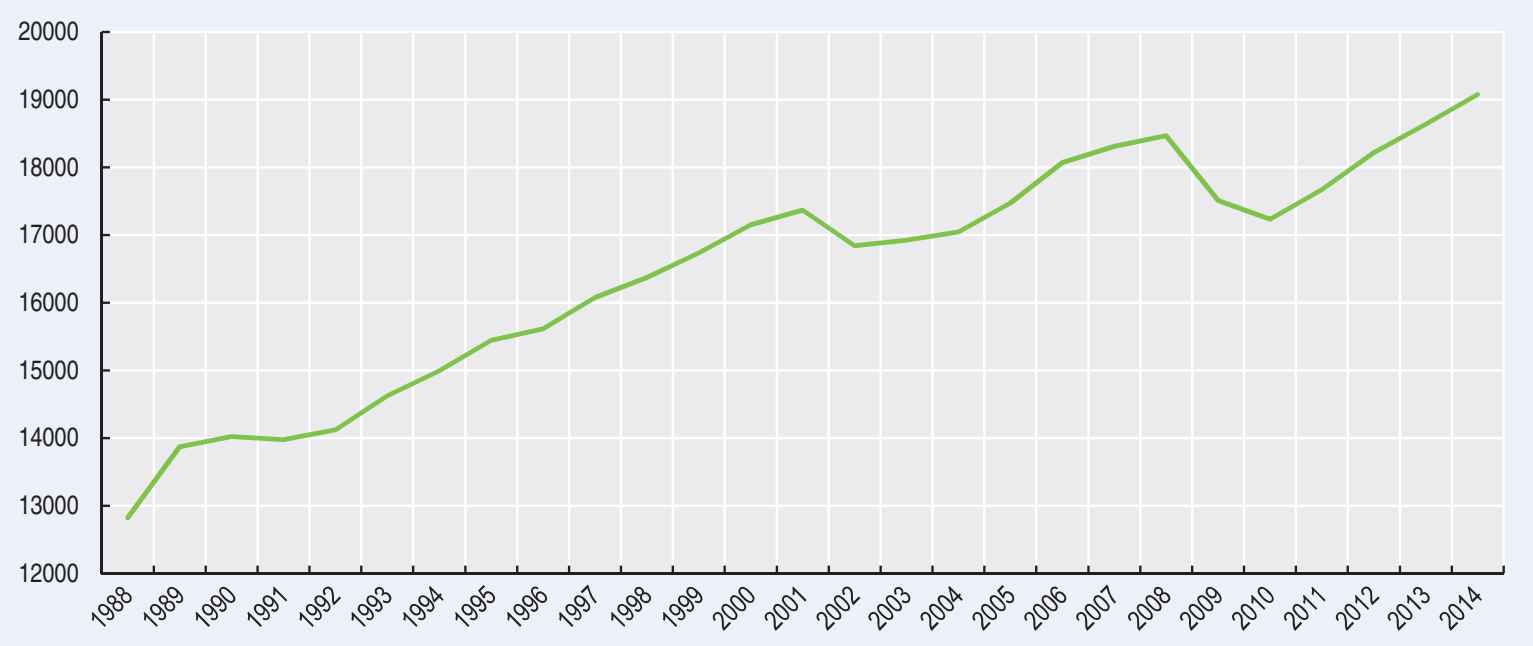

Source : U.S. Small Business Administration 


\section{Encadré 1.1. Gros plan sur la baisse séculaire des taux de créations d'entreprises (suite)}

Mais l'étude du nombre d'établissements sans salarié laisse à penser que l'impact de la hausse de la concentration du marché n'a peut-être pas été particulièrement marqué. Le nombre de ces établissements a ainsi progressé d'environ $60 \%$ au cours des quinze dernières années.

\section{Graphique 1.6. Nombre d'établissements sans salarié aux États-Unis} En millions

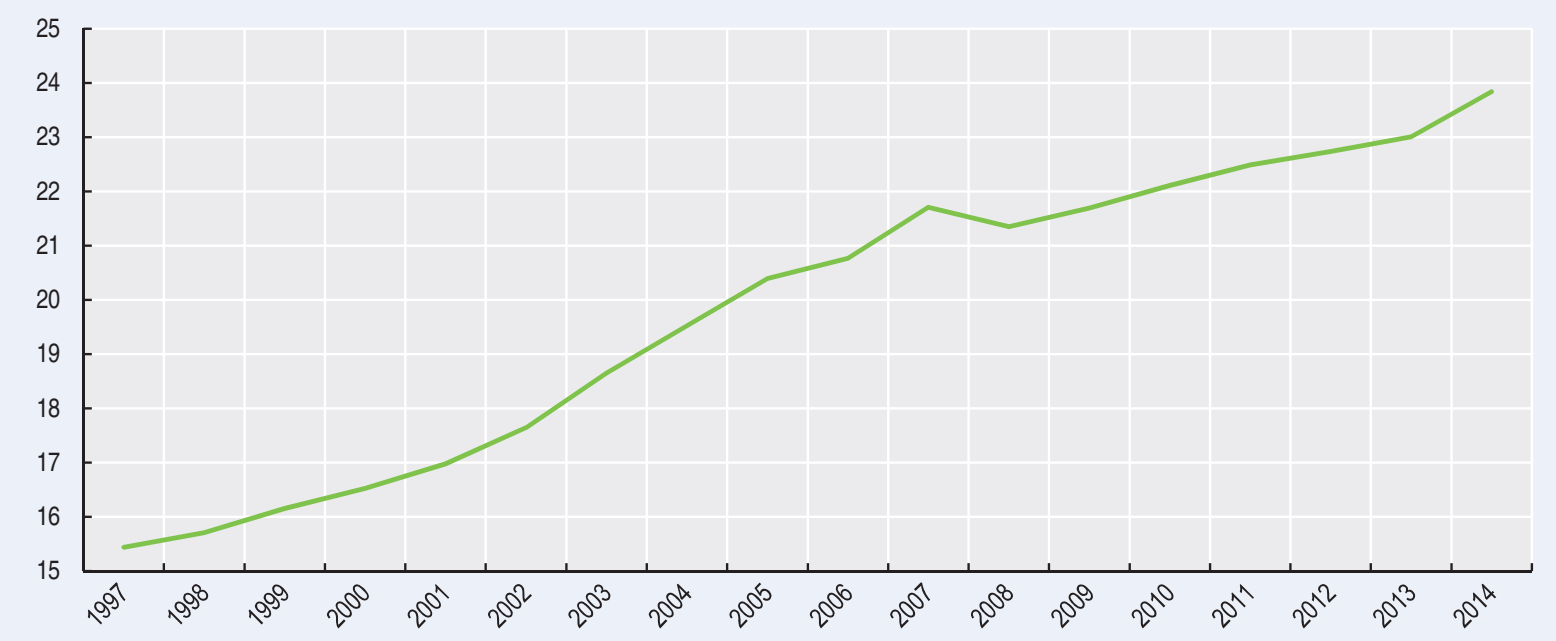

Source : U.S. Small Business Administration

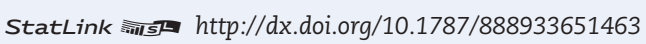

L'analyse de la croissance du nombre d'entreprises au cours de la dernière décennie fait apparaître des évolutions similaires dans de nombreux pays de l'OCDE. Cette croissance a été particulièrement marquée entre 2005 et 2014 dans un grand nombre d'entre eux, et ne s'est contractée que dans les pays sévèrement touchés par la crise (graphique 1.7), ce qui nuance aussi légèrement la thèse de la baisse séculaire.

\section{Graphique 1.7. Croissance du nombre d'entreprises, 2005-2014}

\section{Pourcentage moyen d'évolution annuelle}

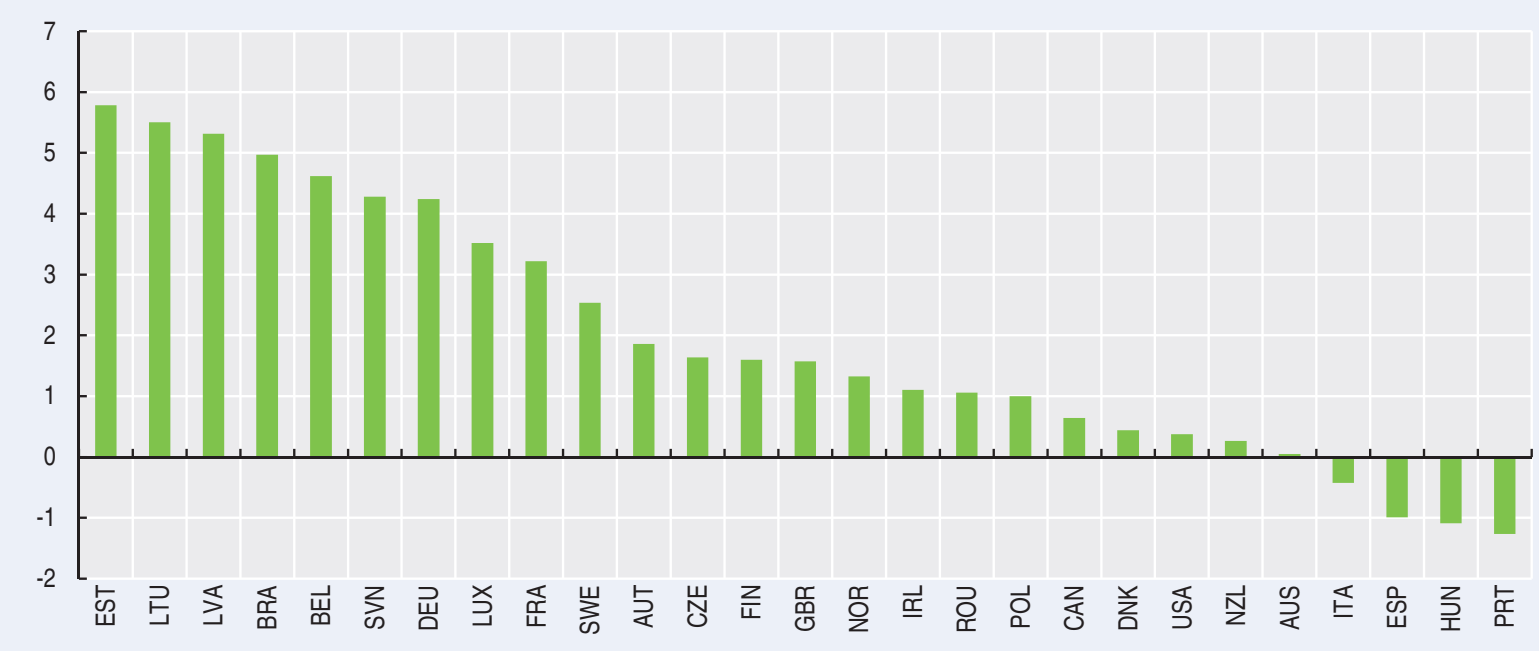

Source : Base de données OCDE sur les statistiques structurelles et démographiques des entreprises. 


\section{Encadré 1.1. Gros plan sur la baisse séculaire des taux de créations d'entreprises (suite)}

Aucun des éléments ci-dessus, toutefois, ne vient complètement remettre en cause la thèse de la baisse séculaire. Les chiffres présentés, par exemple, ne font qu'illustrer les variations nettes de création d'entreprises déduction faite des fermetures. L'accroissement de la population d'entreprises peut certainement aller de pair avec un recul du nombre de créations et une réduction du nombre de faillites, et donc avec des niveaux plus faibles de destruction créatrice et par extension d'entrepreneuriat. Toutefois, un gros plan sur le nombre d'entreprises actives, qui a sensiblement progressé dans de nombreux pays malgré une diminution du nombre de start-up, permet d'apporter un éclairage supplémentaire et pourrait suggérer que la situation de l'entrepreneuriat au sens le plus large est moins morose que ne le laissent à penser les seuls taux de créations d'entreprises.

Il convient de souligner un dernier aspect statistique, concernant la comparaison des taux de création d'entreprises et de la croissance des entreprises entre pays, qui nécessite de tenir également compte de la taille du pays considéré. L'indicateur utilisé à des fins de comparaisons internationales et prôné dans cette publication est celui « d'entreprise ». Lorsqu'une entreprise américaine basée à New York crée un nouvel établissement en Californie, on considère qu'il y a croissance, mais lorsqu'une entreprise, située admettons en France, crée un nouvel établissement (et donc une " entreprise ") au Luxembourg, on considère qu'il y a création d'entreprise au Luxembourg mais pas de hausse de croissance en France.

\section{Le nombre de faillites est revenu à son niveau d'avant la crise}

Parallèlement au rebond des créations d'entreprises, on observe logiquement une amélioration sur le front des faillites. À la fin de 2016, le nombre de faillites était revenu à son niveau dıavant la crise, voire en deçà, dans la plupart des pays. Il est toutefois resté supérieur aux niveaux de 2007 en Espagne, en Islande et en Italie, même si les dernières tendances observées en glissement trimestriel au début de l'année 2017 laissent entrevoir une amélioration dans ces trois pays. 


\section{Graphique 1.8. Faillites, échantillon de pays}

Tendance-cycle, $2012=100$

Toutes formes juridiques confondues

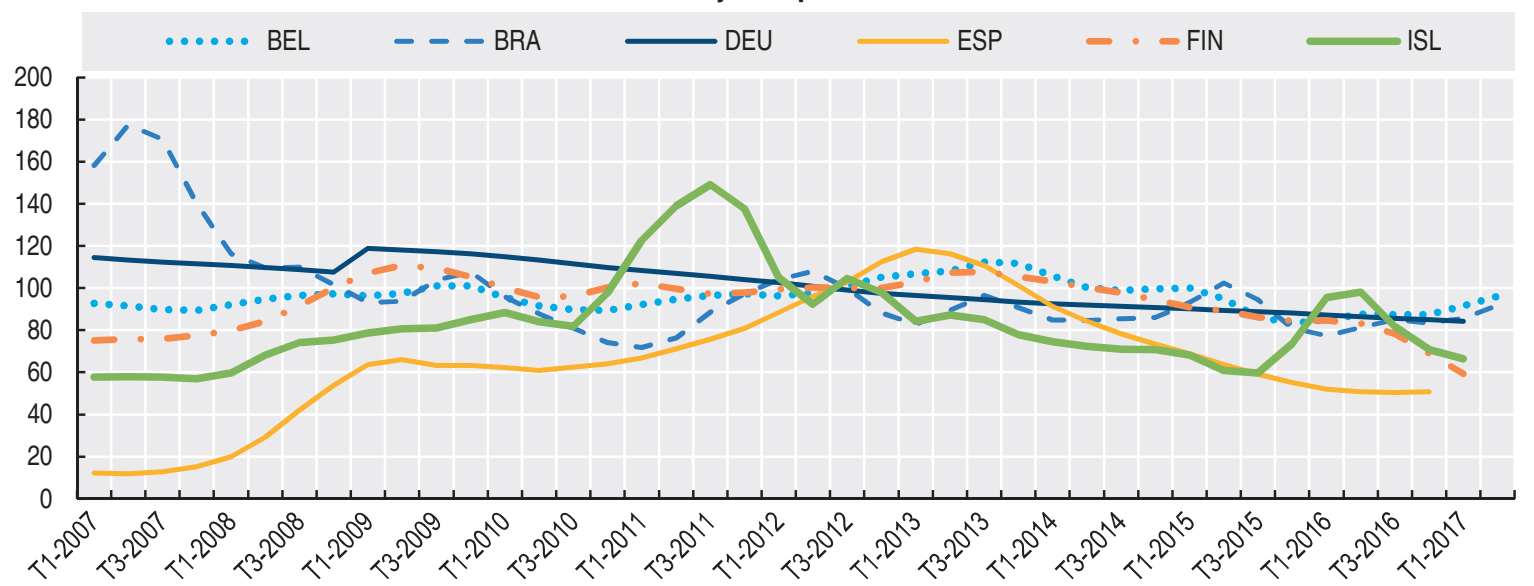

Toutes formes juridiques confondues

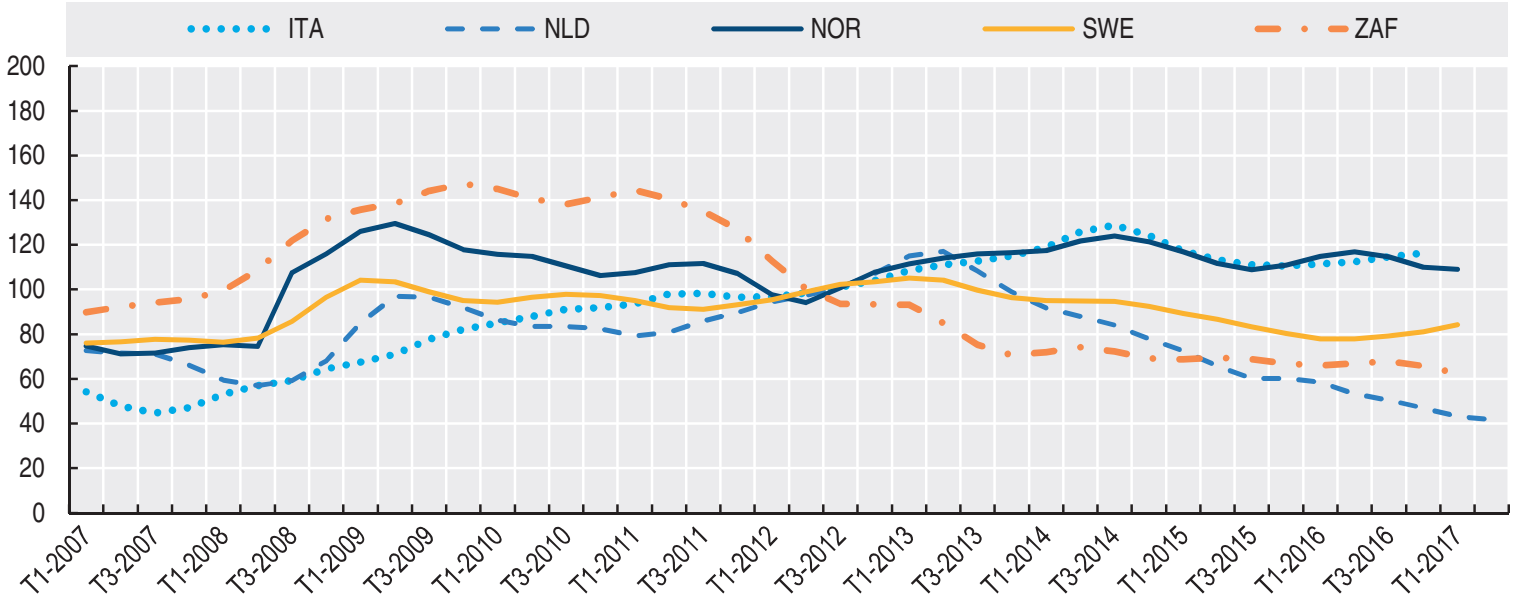

Sociétés

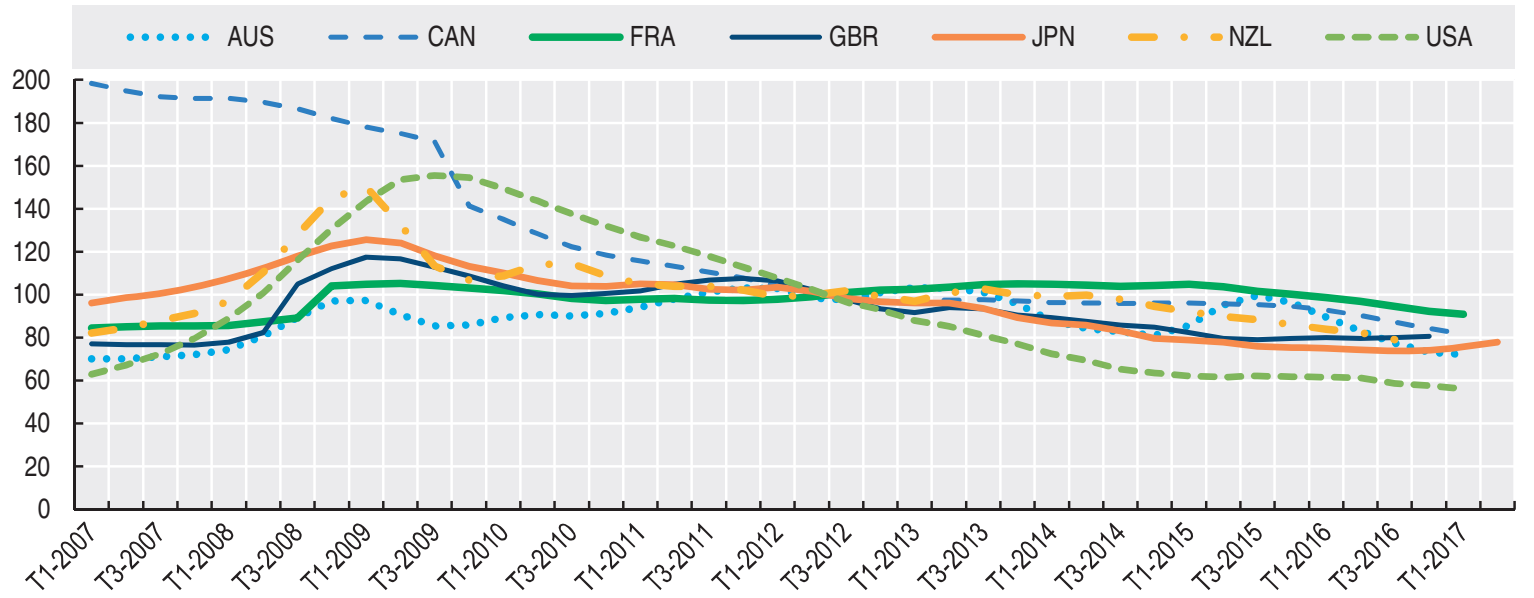

Source : Base de données de l'OCDE concernant les indicateurs actualisés de l'entrepreneuriat, juillet 2017. 


\section{Le travail indépendant a évolué différemment selon les pays}

Si les tendances observées sur le front des start-up et sur celui des faillites commencent à converger dans la plupart des économies, le travail indépendant (effectué par des personnes qui possèdent leur propre entreprise) continue de suivre des trajectoires différentes selon les pays (graphique 1.9). Dans un grand nombre d'entre eux, notamment en Allemagne, en Australie, en Corée, aux États-Unis, en Italie, en Pologne et en Suède, les taux de travail indépendant continuent de diminuer, même si le nombre de travailleurs indépendants reste stable, ce qui vient renforcer les messages mis en avant dans l'encadré 1.1, à savoir qu'il convient de faire la distinction entre taux et niveaux. En Grèce, au Japon et au Portugal, en revanche, la chute des taux de travail indépendant a coïncidé avec un net recul du nombre de travailleurs indépendants.

À l'inverse, les taux de travail indépendant et le nombre de travailleurs indépendants ont atteint en 2016 des niveaux nettement supérieurs à ceux d'avant la crise aux Pays-Bas et au Royaume-Uni, avec une orientation elle aussi nettement à la hausse. Ces deux valeurs étaient aussi sensiblement plus élevées que celles d'avant la crise en Finlande et en France, mais les tendances récentes indiquent un fléchissement dans ces deux pays.

Malgré l'évolution différente du travail indépendant selon les pays, une même tendance se dégage dans la plupart d'entre eux, à savoir la hausse du nombre de travailleurs indépendants à temps partiel et de leur part dans le travail indépendant (graphique 1.10). Dans bon nombre des pays de l'OCDE, le travail indépendant à temps partiel a considérablement progressé au cours des dix dernières années et reflète en partie les nouvelles perspectives offertes par l'essor de «l'économie à la demande » dans plusieurs d'entre eux (OCDE, 2016). En fait, le nombre réel de personnes physiques actives dans l'économie à la demande est probablement plus élevé que ne l'indiquent les chiffres ci-après, qui couvrent uniquement les personnes qui déclarent le travail indépendant à temps partiel comme activité principale, mais ne tiennent pas compte de celles qui ont un emploi salarié et exercent une activité indépendante comme seconde source de revenu (voir chapitre 6).

L'émergence des « travailleurs à la demande " amène à se demander s'il est judicieux d'utiliser la proportion de travailleurs indépendants ou leur nombre comme variable indicatrice de l'ampleur de l'entrepreneuriat (encadré 1.2). On sait depuis longtemps qu'il est nécessaire d'être prudent en la matière, tout particulièrement en ce qui concerne les travailleurs indépendants qui exercent une activité à faible croissance de pure subsistance et ceux contraints par nécessité d'exercer une activité indépendante, mais avec l'émergence de l'économie à la demande, cette question se pose avec encore plus d'acuité. Bien souvent, dans l'économie à la demande, les travailleurs se distinguent très peu des salariés classiques, la seule différence étant qu'ils ont moins facilement accès aux droits et avantages dont bénéficient généralement ces derniers, ce qui les amène dans certains pays à intenter des actions en justice pour contester leur statut de travailleur indépendant (voir dans Sundararajan, 2015, et Balaram et al., 2017, un exposé de la polémique autour du statut des travailleurs de l'économie à la demande et son incidence sur la fiscalité et la protection sociale). 


\section{Graphique 1.9. Travail indépendant, échantillon de pays}

Tendance-cycle, $2012=100$

\section{Nombre d'emplois indépendants}
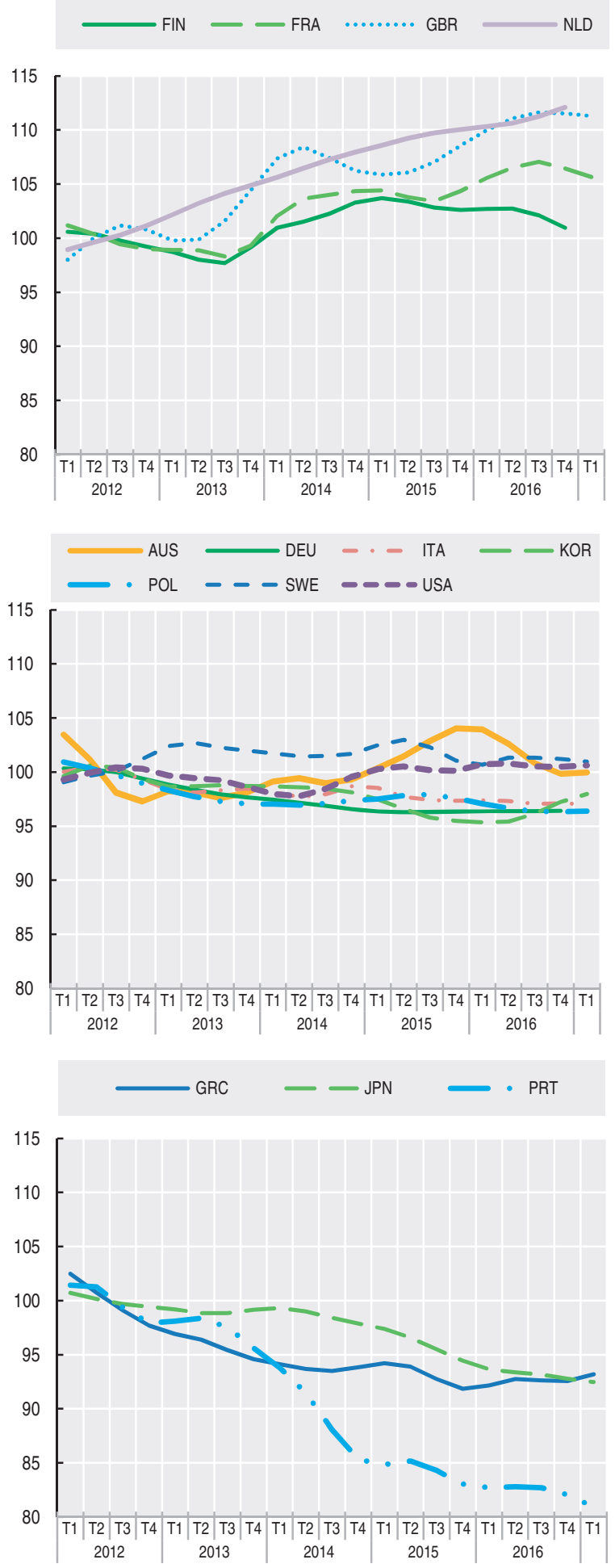

Taux de travail indépendant
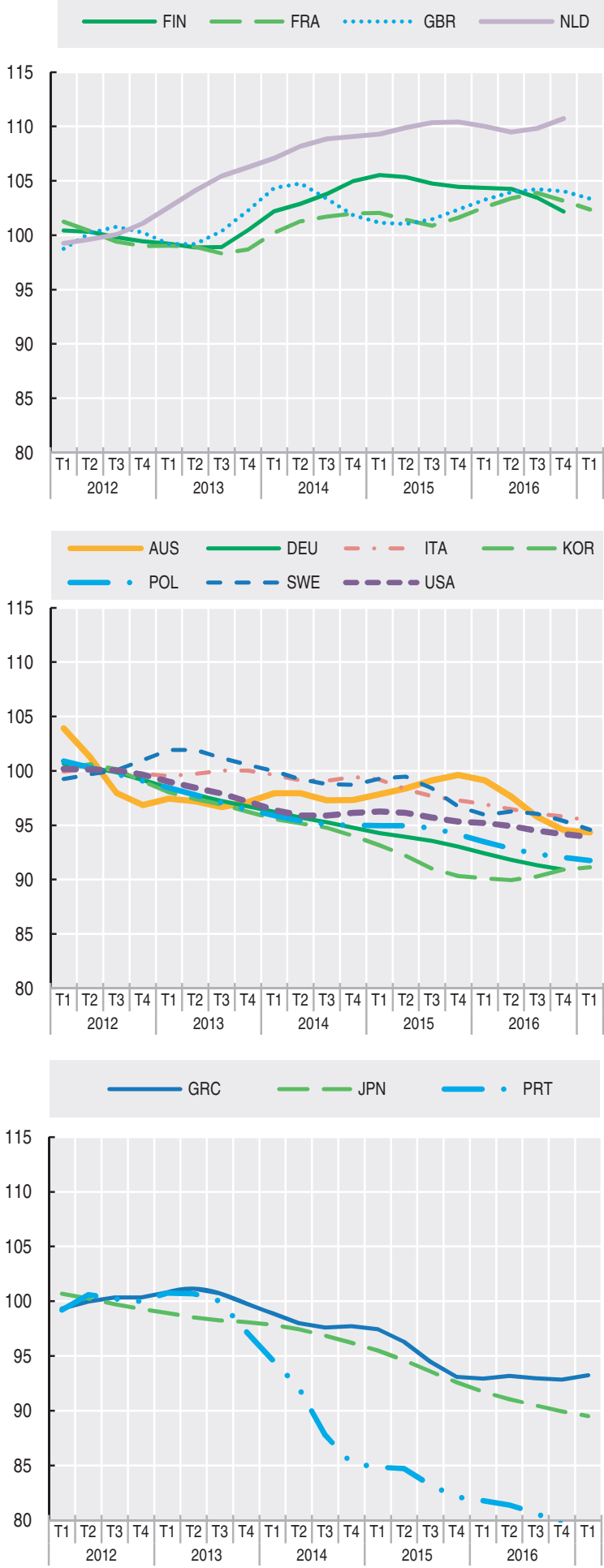

Source : Eurostat : Enquête sur les forces de travail, http://ec.europa.eu/eurostat/web/microdata/european-union-labour-force-survey ; Japon : Enquête sur la population active, http://www.e-stat.go.jp/SG1/estat/eStatTopPortalE.do ; États-Unis : Current Population Survey, www.census. gov/cps/. 
Graphique 1.10. Travail indépendant à temps partiel

Nombre de travailleurs indépendants à temps partiel $(2005=100)$

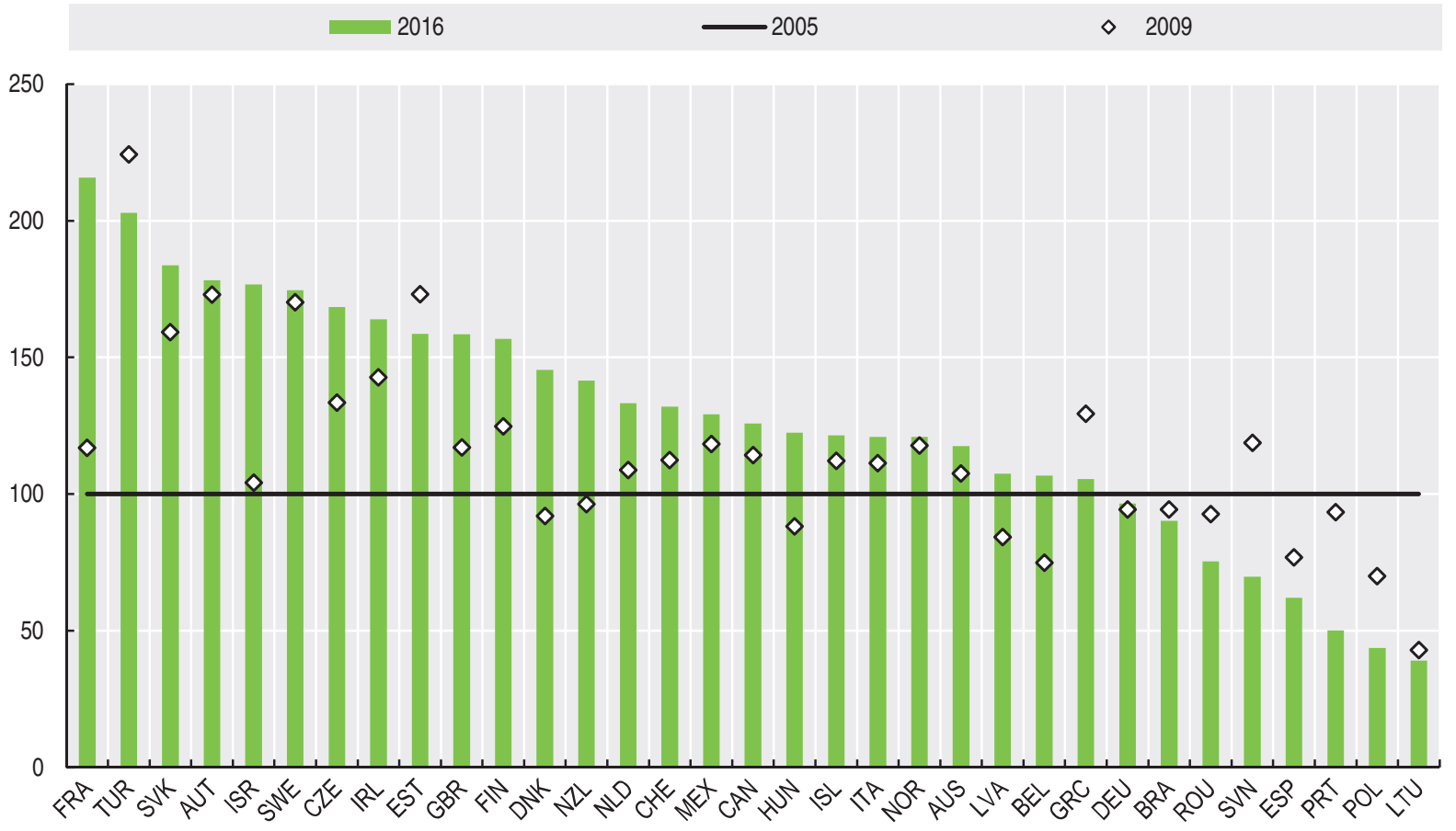

Pourcentage des travailleurs indépendants à temps partiel dans le total des travailleurs indépendants

- $2016 \quad 02005 \quad \diamond 2009$

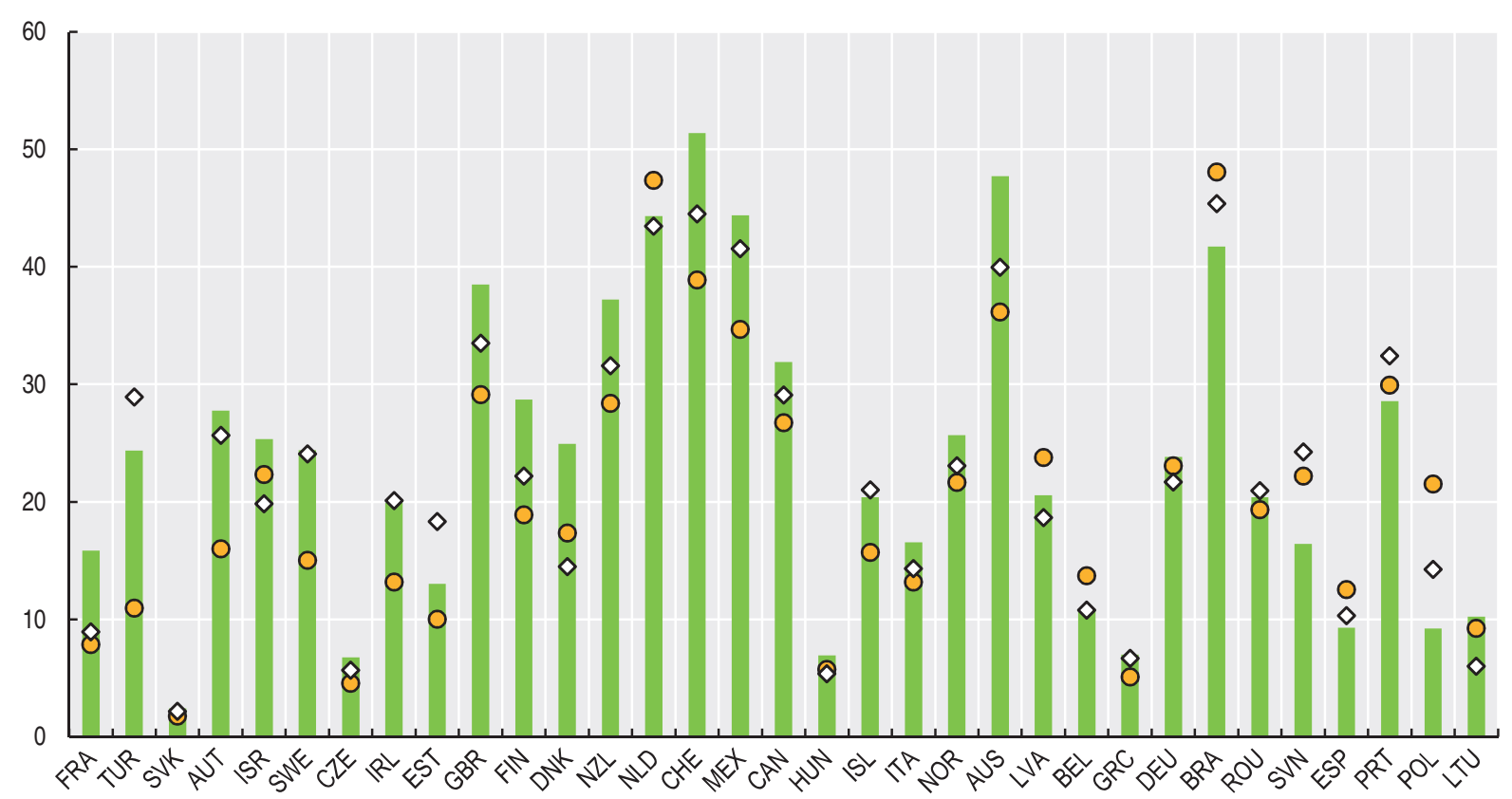

Source : OCDE (2017), «Statistiques du marché du travail : Emploi à plein temps et à temps partiel - définition commune ", Statistiques de l’OCDE sur l'emploi et le marché du travail (base de données), http://dx.doi.org/10.1787/data-00298-en. 


\section{Encadré 1.2. L'économie à la demande est-elle favorable à l'entrepreneuriat ?}

L'expression « économie à la demande » est généralement utilisée pour désigner l'essor de formes d'emploi flexibles, ou du " travail à la tâche ", qui concerne de plus en plus de personnes. Si le concept n'est pas formellement défini, une étude récente menée au Royaume-Uni fait référence à la « tendance à recourir à des plateformes en ligne pour dénicher des petits boulots, qui sont parfois effectués dès la requête soumise (essentiellement à la demande) " (Balaram et al., 2017). Ces formes d'emploi flexibles complètent les emplois à temps plein ou s'y substituent, et permettent aussi aux personnes sans emploi de mettre un pied dans la vie active. Le travail à la tâche n’a en soi rien de nouveau, puisqu'il a toujours représenté pour certains secteurs, comme l'industrie du spectacle, par exemple, une importante source de revenu, mais il est aujourd'hui proposé et demandé par des personnes de plus en plus nombreuses et variées et couvre un éventail de services plus large que jamais.

La montée en puissance rapide du travail à la tâche est alimentée par la technologie et largement associée à l'essor des plateformes en ligne, comme Uber et TaskRabbit, qui mettent en relation acheteurs et vendeurs pour des transactions ponctuelles.

L'économie à la demande étant relativement récente, on ne dispose généralement pas de données d'évaluation sur le nombre de " travailleurs à la tâche " dans les pays de l'OCDE. Balaram et al. (2017) ont estimé qu'ils étaient environ 1.1 million au Royaume-Uni, que seulement $12 \%$ d'entre eux travaillaient tous les jours, et seulement $8 \% 35$ heures ou plus par semaine

Le lien entre l'économie à la demande et l'activité entrepreneuriale est loin d'être évident. Les acteurs de l'économie à la demande peuvent être de petits entrepreneurs : sur la plate-forme Etsy, par exemple, les artisans peuvent facilement commercialiser dans le monde entier leurs bijoux, vêtements ou accessoires faits main. Mais bon nombre d'entre eux ne sont pas nécessairement des entrepreneurs au sens strict, en ce sens qu'ils peuvent s'être engagés contractuellement à fournir leurs services exclusivement à une entreprise et présenter de fortes similitudes avec les salariés classiques. D'un autre côté, la flexibilité associée au travail à la tâche contraste souvent avec l'emploi salarié traditionnel, et peut encourager les entrepreneurs en herbe à réaliser leurs projets de nouvelles activités tout en restant en mesure de subvenir à leurs besoins (en fait, cette vision est souvent mise en avant par les plateformes numériques de mise en relation).C'est pourquoi, il est difficile d'apprécier directement l'impact du travail à la tâche lorsque l'on s'efforce de prendre la mesure de l'entrepreneuriat.

En outre, certains éléments laissent à penser que l'économie à la demande peut parfois peser sur l'activité entrepreneuriale. Burtch et al. (2016), par exemple, ont étudié l'impact de la pénétration des marchés locaux par Uber (service de chauffeurs) et Postmates (service de livraison à la demande) sur le niveau de l'entrepreneuriat local, tel que mesuré par le volume de campagnes de financement participatif lancées sur Kickstarter, principale plateforme dans ce domaine au monde. Les auteurs ont estimé que le volume des campagnes avait sensiblement chuté, et que cette baisse reflétait essentiellement une diminution du nombre de campagnes non abouties. Ce qui les a amenés à conclure que l'économie à la demande venait essentiellement se substituer à un entrepreneuriat de qualité médiocre, et non compléter un entrepreneuriat de qualité élevée.

\section{Le numérique ouvre de nouvelles perspectives et de nouveaux marchés qui favorisent la croissance de l'entrepreneuriat}

Le développement d'outils et de plateformes numériques abordables a offert aux microentreprises de nouvelles possibilités d'accéder aux marchés étrangers d'une façon qui aurait été précédemment inimaginable.

Les nouvelles données de l'enquête sur l'avenir des entreprises (Future of Business Survey), réalisée conjointement par Facebook, l'OCDE et la Banque mondiale, montrent que même les structures unipersonnelles (c'est-à-dire composées d'un travailleur indépendant sans 
salarié), malgré leur petite taille, peuvent réaliser une part essentielle de leurs activités à l'exportation, en tirant profit des outils numériques (Facebook, OCDE, Banque mondiale, 2017). Par le passé, seules les grandes multinationales pouvaient, concrètement, acquérir une présence mondiale. De nos jours, les petites entreprises ont à leur disposition toute une palette d'outils numériques qui leur permet de mobiliser les réseaux et de commercialiser directement leurs produits et services auprès de clients potentiels dans le monde entier, et, partant, de surmonter les obstacles aux échanges qui pèsent traditionnellement davantage sur les petites entreprises caractérisées par de plus faibles économies d'échelle.

Si le pourcentage de petites et moyennes entreprises (PME) présentes à l'international varie fortement d'un pays et d'une région à l'autre, dans la plupart des économies, 20 \% environ des PME présentes sur l'internet interrogées entre mars et mai 2017 déclarent exercer leurs activités à l'international ; 6 \% d'entre elles sont à la fois importatrices et exportatrices, $5 \%$ seulement exportatrices et $8 \%$ exclusivement importatrices (graphique 1.11). Aux fins de cette enquête, les PME sont définies comme les entreprises ayant moins de 250 salariés. Les écarts entre pays s'expliquent en partie par la représentativité plus ou moins grande des entreprises participantes. L'enquête, par sa conception même, ne couvre que les entreprises présentes sur Facebook, et cette cohorte reflète probablement mieux la population générale d'entreprises dans les pays avancées que dans les économies en développement et émergentes.

\section{Graphique 1.11. Commerce international et PME présentes sur l'internet}

Part dans le total des répondants à l'enquête, mars-mai 2017

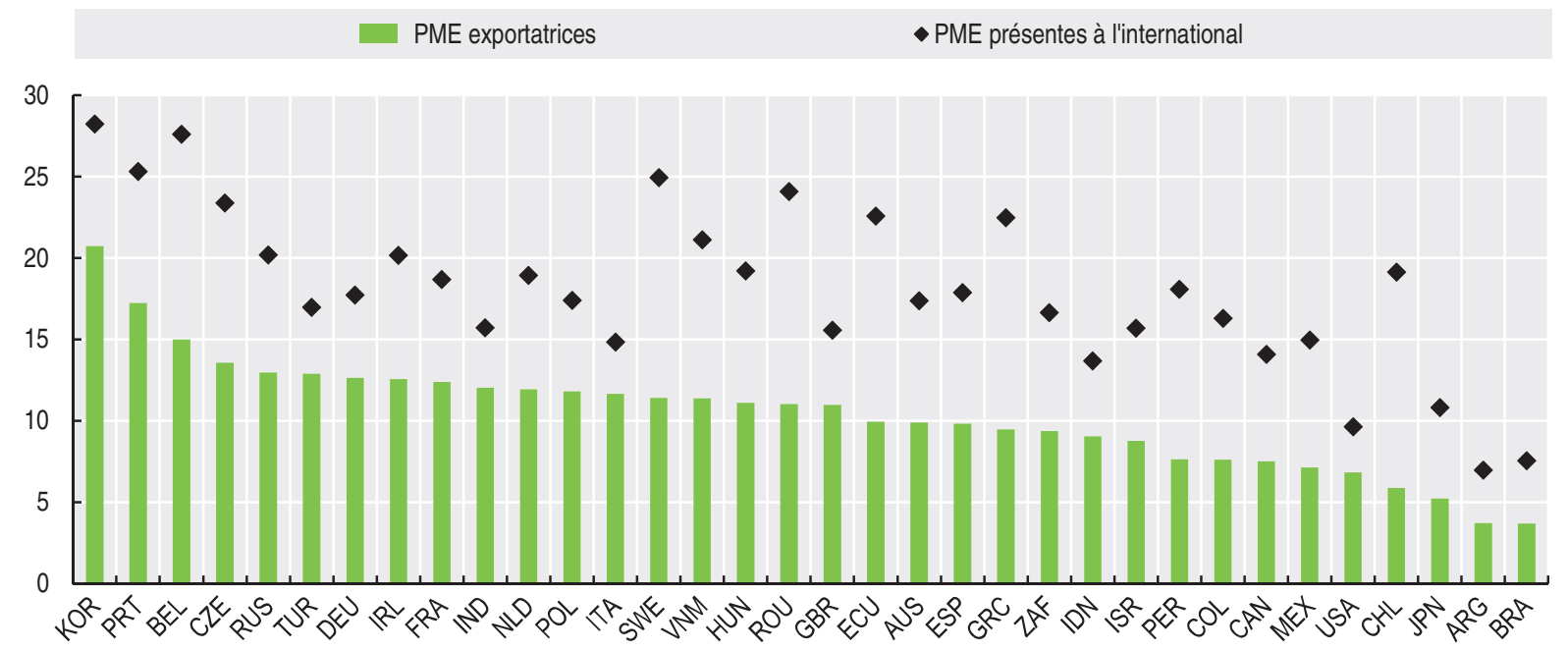

Note : réponses fournies par les entreprises ayant une page Facebook entre mars et mai 2017. Les entreprises présentes à l'international sont définies comme les entreprises importatrices et/ou exportatrices, tandis que les entreprises exportatrices sont à la fois exportatrices et importatrices ou seulement exportatrices.

Source : Facebook-OCDE-Banque mondiale, Future of Business Survey (base de données), juin 2017

StatLink 完正田 http://dx.doi.org/10.1787/888933651558

L'enquête révèle que les exportations représentent une composante essentielle du modèle économique des entreprises exportatrices, non seulement pour une proportion considérable de petites entreprises (moins de 50 salariés), mais également pour bon nombre des structures unipersonnelles. Près d'un tiers $(28 \%)$ de ces dernières qui exportent déclarent réaliser plus de $25 \%$ de leurs recettes totales à l'international (graphique 1.12). En outre, deux tiers des PME exportatrices indiquent que plus de $50 \%$ de leurs ventes à l'international se font en ligne, les PME manufacturières étant celles qui exportent le plus, suivies des PME présentes dans le commerce de gros et de détail. 


\section{Graphique 1.12. Entreprises dont les recettes d'exportation sont supérieures à $25 \%$ du total des recettes, par taille d'entreprise \\ Pourcentage d'entreprises exportatrices, mars-mai 2017}

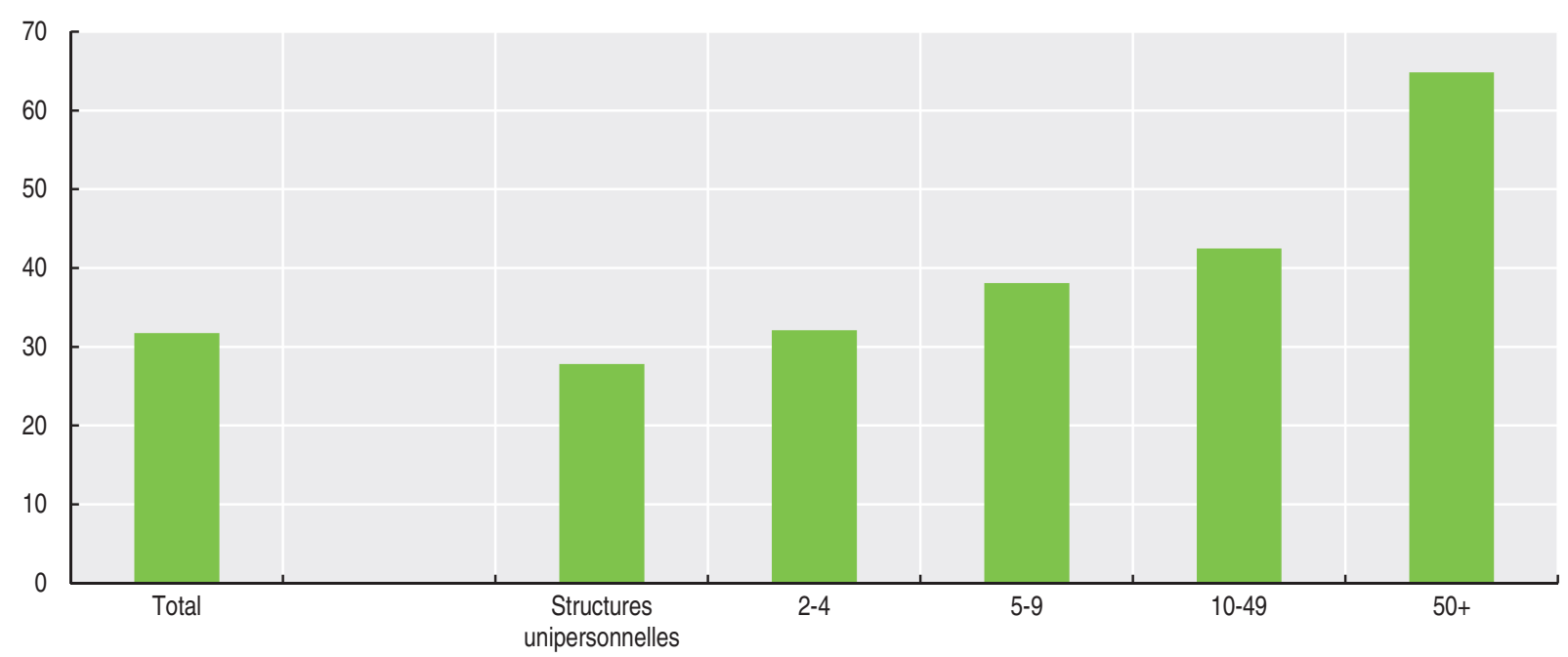

Note: réponses fournies par les entreprises ayant une page Facebook entre mars et mai 2017. Les entreprises présentes à l'international sont définies comme les entreprises importatrices et/ou exportatrices, tandis que les entreprises exportatrices sont à la fois exportatrices et importatrices ou seulement exportatrices.

Source : Facebook-OCDE-Banque mondiale, Future of Business Survey (base de données), juin 2017.

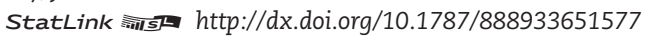

Les données les plus récentes de l'enquête sur l'avenir des entreprises (Future of Business Survey) confirment également des conclusions antérieures sur le lien entre confiance des entreprises et commerce international. Les entreprises présentes à l'international sont plus confiantes quant à leur situation actuelle et à leur avenir, et sont aussi généralement plus optimistes quant aux perspectives de créations d'emplois (graphiques 1.13 et 1.14). Cela vaut également pour les structures unipersonnelles, même si les grandes entreprises ont généralement une vision plus positive de leur situation présente et future et des perspectives en matière de créations d'emplois. 


\section{Graphique 1.13. Présence sur l'internet, commerce international et confiance des entreprises}

Pourcentage de réponses positives parmi les répondants à l'enquête, mars-mai 2017

\section{Situation actuelle}

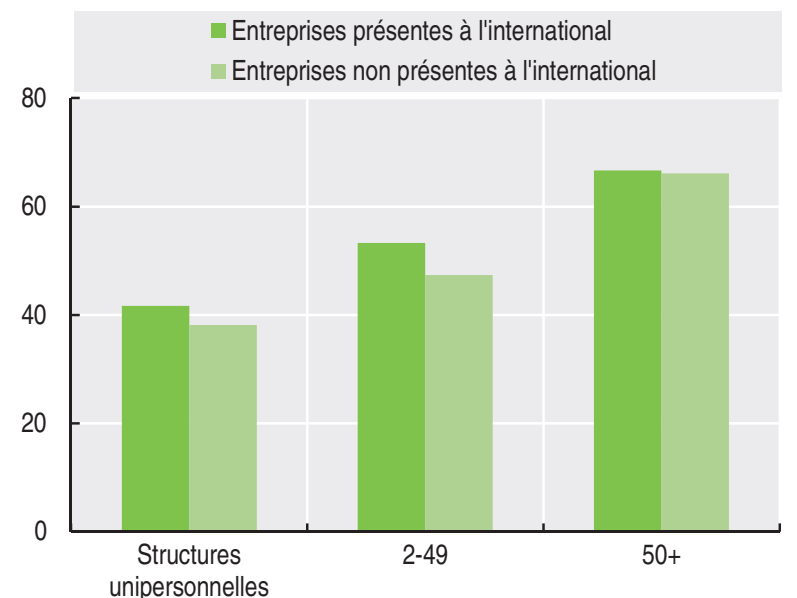

Perspectives

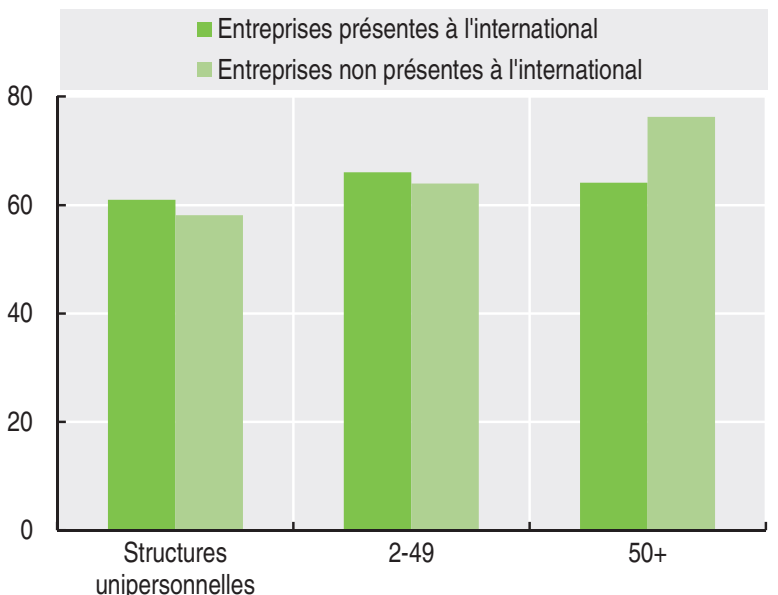

Note: réponses fournies par les entreprises ayant une page Facebook entre mars et mai 2017. Les entreprises présentes à l'international sont définies comme les entreprises importatrices et/ou exportatrices, tandis que les entreprises exportatrices sont à la fois exportatrices et importatrices ou seulement exportatrices. Dans "Situation actuelle" » et "Perspectives ", les chiffres sont calculés à partir des réponses " Positive(s) » aux questions suivantes : "Comment évaluez-vous la situation actuelle de votre entreprise? » et "Quelles sont les perspectives de votre entreprise dans les six prochains mois? ".

Source : Facebook-OCDE-Banque mondiale, Future of Business Survey (base de données), juin 2017.

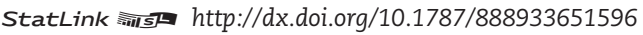

\section{Graphique 1.14. Présence sur l'internet, commerce international et perspectives de créations d'emplois}

Pourcentage de réponses positives parmi les répondants à l'enquête, mars-mai 2017
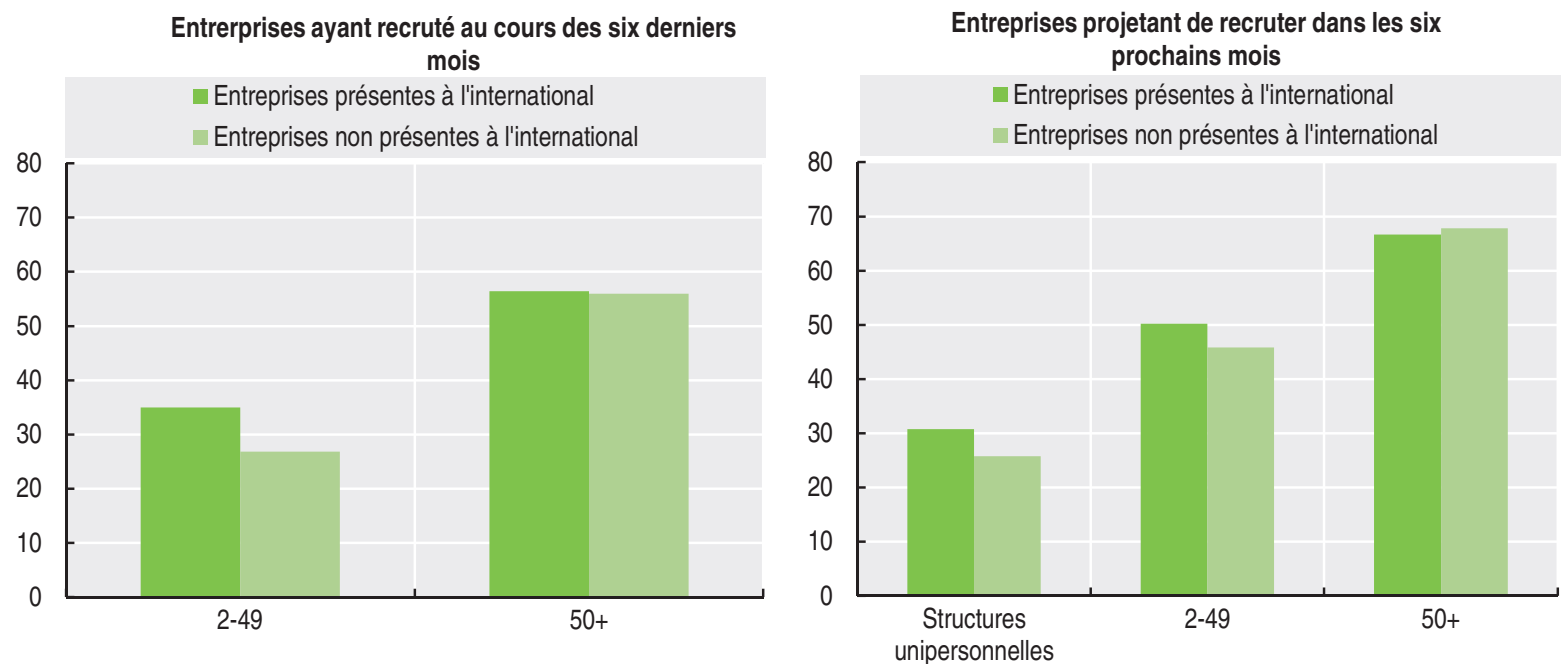

Note : réponses fournies par les entreprises ayant une page Facebook entre mars et mai 2017. Les entreprises présentes à l'international sont définies comme les entreprises importatrices et/ou exportatrices, tandis que les entreprises exportatrices sont à la fois exportatrices et importatrices ou seulement exportatrices. Les chiffrent sont calculés à partir des réponses "Augmentation " aux questions suivantes : "Comment a évolué le nombre d'employés dans votre entreprise au cours de six derniers mois ? " et "Comment envisagez-vous lévolution du nombre d`employés dans votre entreprise dans les six prochains mois ? ".

Source : Facebook-OCDE-Banque mondiale, Future of Business Survey (base de données), juin 2017. 
La création d'une entité exportatrice peut, avec l'ouverture de nouveaux marchés, jouer un rôle essentiel dans la réussite et la croissance d'une entreprise. Cependant, près de la moitié des PME exportatrices jugent difficile de réaliser des ventes à l'étranger (d'autant plus peut-être pour celles qui souhaitent exporter mais qui n'ont pas été jusqu'à présent en mesure de le faire). Les principaux obstacles à l'exportation cités sont, entre autres, la recherche de partenaires commerciaux, les limitations d'accès aux marchés et la réglementation. Surmonter de telles difficultés constitue pour ces entreprises un facteur clé de réussite.

\section{Sources et pour en savoir plus}

Balaram, B., Warden J. et F. Wallace-Stephens (2017), Good Gigs. A fairer future for the UK's gig economy, RSA (Royal Society for the encouragement of Arts, Manufactures and Commerce), avril, https://www. thersa.org/globalassets/pdfs/reports/rsa_good-gigs-fairer-gig-economy-report.pdf.

Bartelsman, E.J., S. Scarpetta et F. Schivardi (2003), « Comparative Analysis of Firm Demographics and Survival: Micro-Level Evidence for the OECD Countries ", Document de travail du Département des affaires économiques de l’OCDE $n^{\circ} 348$, OCDE, Paris.

Burtch, G., Carnahan, S. et B. N. Greenwood (2016), « Can You Gig it? An Empirical Examination of the Gig-Economy and Entrepreneurial Activity ", Document de travail de l'Université du Michigan, https:// papers.ssrn.com/sol3/papers.cfm?abstract_id=2744352.

Blanchenay, P., Criscuolo, C. et F. Calvino (à paraître), « Cross-country evidence on business dynamics over the last decade: from boom to gloom? ", OECD Science, Technology and Industry Working Papers, Éditions OCDE, Paris.

Decker, R., Haltiwanger J., Jarmin R. et J. Miranda (2016), « Declining Business Dynamism: What We Know and the Way Forward ", The American Economic Review.

Facebook-OCDE-Banque mondiale (2017), The Future of Business Survey. Trade Report, juillet, www. futureofbusinesssurvey.org.

Fairlie, R. W., A. Morelix, E. J. Reedy et J. Russell (2016). 2016 - The Kauffman Index of Startup Activity. National trends. Kansas City: Ewing Marion Kauffman Foundation.

Haltiwanger, J. (2016), " Top ten signs of declining business dynamism and entrepreneurship in the United States ", essay for the New Entrepreneurial Growth Agenda, Kauffman Foundation, http:// www.kauffman.org/neg/section-3.

OCDE (2014), « Statistical Business Registers and Business Demography Statistics: Review of country practices ", document établi pour la $24^{\mathrm{e}}$ réunion du Groupe de Wiesbaden sur les registres d'entreprises. http://www.statistik.at/web_en/about_us/events/wiesbaden_group_meeting_2014/agenda/index.html.

OCDE (2016), «It's a gig, but is it a job? », in L'Observateur de l'OCDE, tiré de http://www.oecdobserver.org/news/ fullstory.php/aid/5464/It_s_a_gig,_but_is_it_a_job_html

Steinmetz, K. (2016), « Exclusive: See How Big the Gig Economy Really Is », in Time Magazine, tiré de http:// time.com/4169532/sharing-economy-poll/

Sundararajan, A. (2015), " The 'gig economy' is coming. What will it mean for work? » in The Guardian, tiré de https://www.theguardian.com/commentisfree/2015/jul/26/will-we-get-by-gig-economy

Telles, R. J. (2016), Digital Matching Firms: A New Definition in the "Sharing Economy" Space, U.S. Department of Commerce, Economics and Statistics Administration, Office of the Chief Economist, https://www. esa.gou/sites/default/files/digital-matching-firms-new-definition-sharing-economy-space.pdf. 

4.463 .72
228.72
285

309281.89

in 300.89 ? 40

$$
\beta
$$

$$
\begin{aligned}
& x^{3}+4^{2}, 05
\end{aligned}
$$

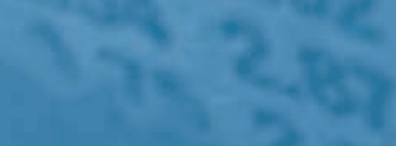
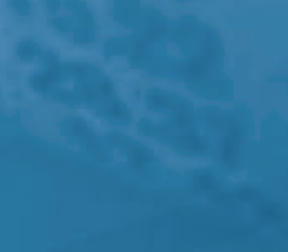

the

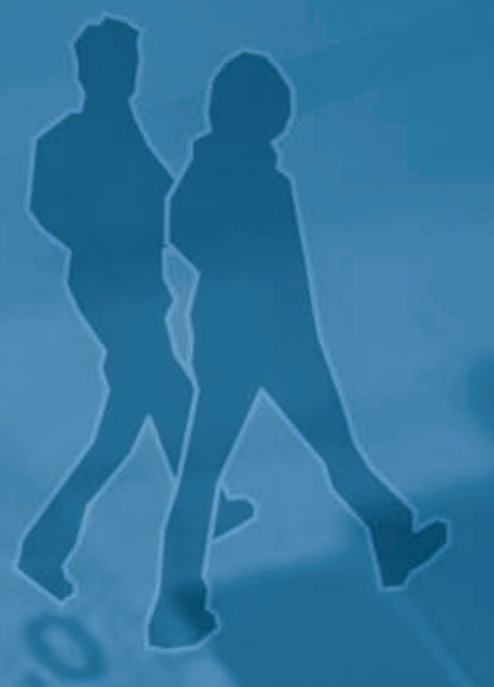

$-6 \times 2$

Q. 35

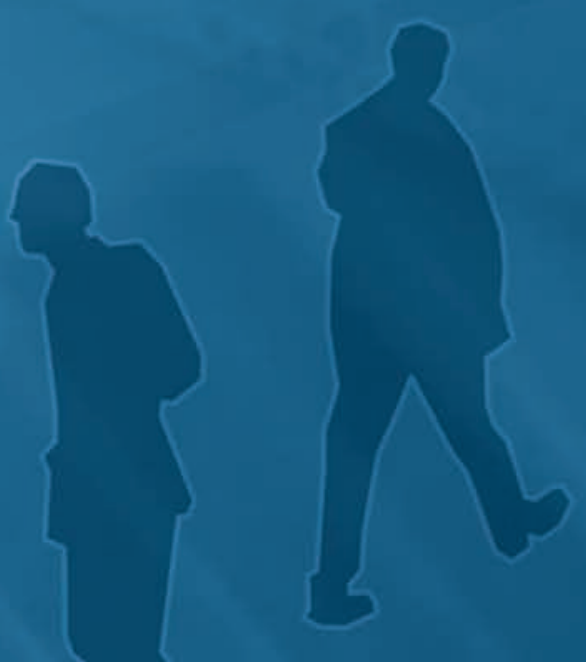




\section{STRUCTURE ET PERFORMANCE DE LA POPULATION DES ENTREPRISES}

Entreprises par classe de taille

Emploi par taille d'entreprise

Valeur ajoutée par taille d'entreprise

Chiffre d'affaires par taille d'entreprise

Rémunération des salariés par taille d'entreprise 


\section{À savoir}

- Dans tous les pays, la grande majorité des entreprises (entre $70 \%$ et $95 \%$ ) sont des microentreprises, c'est-à-dire des entreprises employant moins de 10 personnes, et dans la plupart des pays plus de la moitié des entreprises sont des entreprises sans salarié, par exemple des travailleurs indépendants qui travaillent pour leur propre compte et qui n'emploient personne.

- Du fait notamment des coûts d'entrée élevés et de l'intensité capitalistique du secteur de la fabrication, les PME sont, dans les pays de l'OCDE, beaucoup plus nombreuses dans les secteurs des services, tandis qu'on recense un nombre élevé d'entreprises sans salarié dans le commerce de gros et de détail et dans les activités de construction.

- Dans environ la moitié des économies de l'OCDE, surtout celles durement frappées par la crise, le nombre d'entreprises en 2014 était toujours inférieur au niveau de 2008. Le secteur de la construction est particulièrement touché, et dans une moindre mesure les activités de fabrication, mais les services s'en sortent beaucoup mieux dans la plupart des pays de l'OCDE. Dans tous les secteurs et dans la plupart des économies de l'OCDE, la croissance du nombre de PME a généralement dépassé celle des entreprises de plus grande taille.

\section{Pertinence}

Les petites entreprises peuvent être d'importants moteurs de croissance et d'innovation. Cela étant, en l'absence d'un contexte général approprié, leur croissance peut se heurter à des obstacles, en particulier dans les secteurs à forte intensité capitalistique, où l'accès aux sources de financement et l'intégration dans les chaînes de valeur mondiales sont d'importants facteurs de réussite.

\section{Définitions}

Une entreprise est la plus petite combinaison d'unités légales qui constitue une unité organisationnelle de production de biens et de services jouissant d'une certaine autonomie de décision, notamment pour l'affectation de ses ressources courantes. Une entreprise exerce une ou plusieurs activités dans un ou plusieurs lieux.

Les classes de taille reposent sur le nombre total de personnes occupées, lequel comprend le travailleur indépendant.

Dans cette publication, les microentreprises ont entre 1 et 9 salariés, les petites entreprises entre 10 et 49 salariés, les moyennes entreprises entre 50 et 249 salariés, et les grandes entreprises 250 salariés et plus. Le groupe des microentreprises et des petites et moyennes entreprises (PME) correspond à la classe de taille 1-249.

Le nombre de personnes occupées correspond au nombre total de personnes travaillant pour l'unité d'observation (y compris les propriétaires exploitants, les associés exerçant une activité régulière dans l'unité en question, et les travailleurs familiaux non rémunérés).

Informations sur les données concernant Israël : http:// dx.doi.org/10.1787/888932315602.

\section{Comparabilité}

L'unité statistique sur laquelle reposent les informations présentées est l'entreprise pour tous les pays sauf la Corée et le Mexique, qui utilisent l'établissement. Dans la mesure où la plupart des entreprises y sont constituées d'un seul établissement, cela ne devrait pas poser de problèmes significatifs de comparabilité s'agissant de la population totale d'entreprises mais, par rapport aux autres pays, la proportion de petites entreprises de ces États subira un biais à la hausse et la proportion de grandes entreprises un biais à la baisse.

Compte tenu des pratiques différentes des pays en matière de collecte de données, la ventilation par classe de taille utilisée, à savoir 1-9, 10-19, 20-49, 50-249 et 250+, offre une comparabilité optimale. Certains pays utilisent des conventions différentes : la classe « 1-9 » correspond en réalité à « 1-10 " pour le Mexique et à "1-19 " pour l'Australie et la Turquie ; la classe "10-19" correspond à "11-50 " pour le Mexique ; la classe " 20-49" correspond à la classe " 20-199 " pour l'Australie ; la classe « 50-249 » correspond à « 50-299 " pour la Corée et le Japon, et à « 51-250 " pour le Mexique ; enfin, la classe « 250+ » correspond à « 200+ " pour l'Australie, à « 300+ " pour la Corée et le Japon, et à « 251+ " pour le Mexique.

Pour le Canada, les États-Unis et la Fédération de Russie, les données n'incluent pas les décomptes des entreprises sans salarié. Pour l'ensemble du secteur marchand, le nombre d'entreprises sans salarié s'élève approximativement à 1.7 million au Canada, 15.3 millions aux États-Unis et 2.5 millions dans la Fédération de Russie. Les données pour la Suisse n'incluent pas les entreprises de moins de trois salariés.

Les données concernant la Finlande et le Portugal affichent une rupture de série en 2013 et il en est de même en 2014 pour les données concernant le Canada et la France. Pour le Royaume-Uni, les données présentées n'incluent pas les quelque 2.6 millions de petites entreprises non enregistrées, c'est-à-dire des entreprises n'atteignant pas le seuil prévu pour le régime de la taxe sur la valeur ajoutée et/ou celui de la retenue de l'impôt à la source (pour les entreprises employant des salariés).

Dans le graphique 2.4, les statistiques démographiques des entreprises sont utilisées comme source de données; elles englobent les entreprises sans salarié pour tous les pays, y compris la Suisse.

\section{Sources}

OCDE, Statistiques structurelles et démographiques des entreprises (SDBS) (base de données), http://dx.doi.org/10.1787/sdbs-data-fr.

\section{Pour en savoir plus}

OCDE (2017), Small, Medium, Strong. Trends in SME Performance and Business Conditions, Éditions OCDE, Paris, http://dx.doi. org/10.1787/9789264275683-en.

OCDE (2010), Structural and Demographic Business Statistics, Éditions OCDE, Paris, http://dx.doi.org/10.1787/9789264072886-en.

Ahmad N. (2007), The OECD's Business Statistics Database and Publication, document présenté à la réunion d'experts sur les statistiques structurelles des entreprises, Paris, 10 et 11 mai 2007, www.oecd.org/dataoecd/59/34/38516035.pdf. 
Graphique 2.1. Nombre d'entreprises par taille et principaux secteurs, échantillon de pays $2008=100$

\section{Secteur marchand - PME}

$\begin{array}{ll}\text { CAN } & - \text { - FRA } \\ \cdots \text { ITA } & -- \text { ITA }\end{array}$

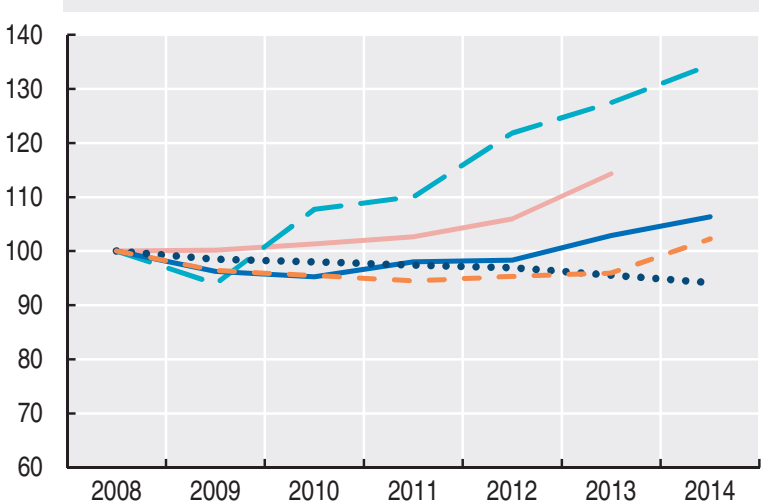

Activités de fabrication - PME

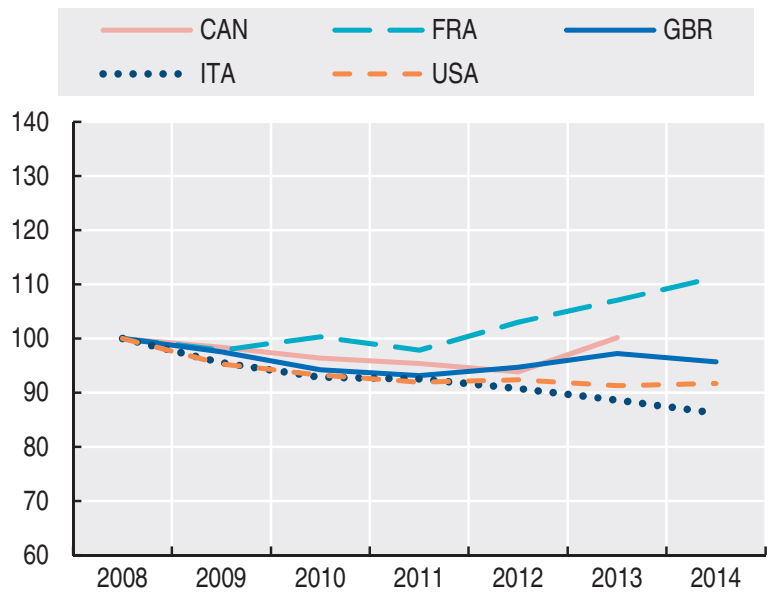

Services - PME
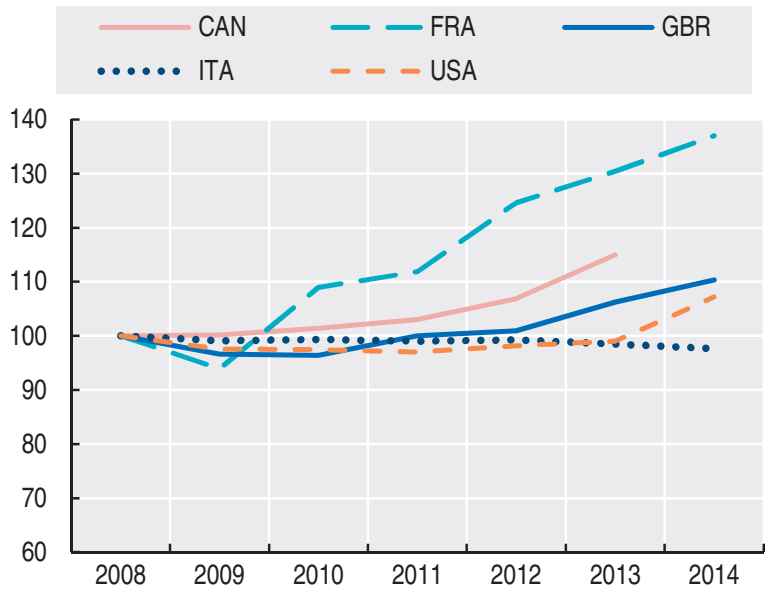

\section{Secteur marchand - Grandes entreprises}
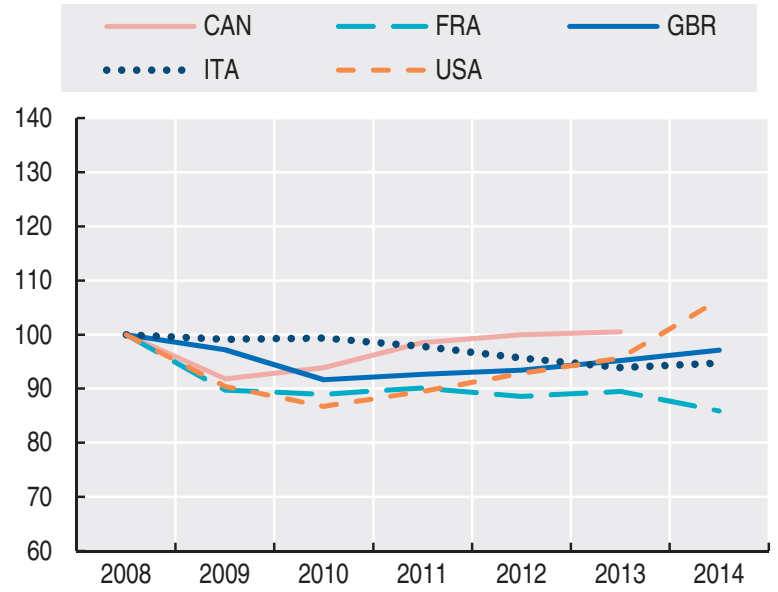

Activités de fabrication - Grandes entreprises
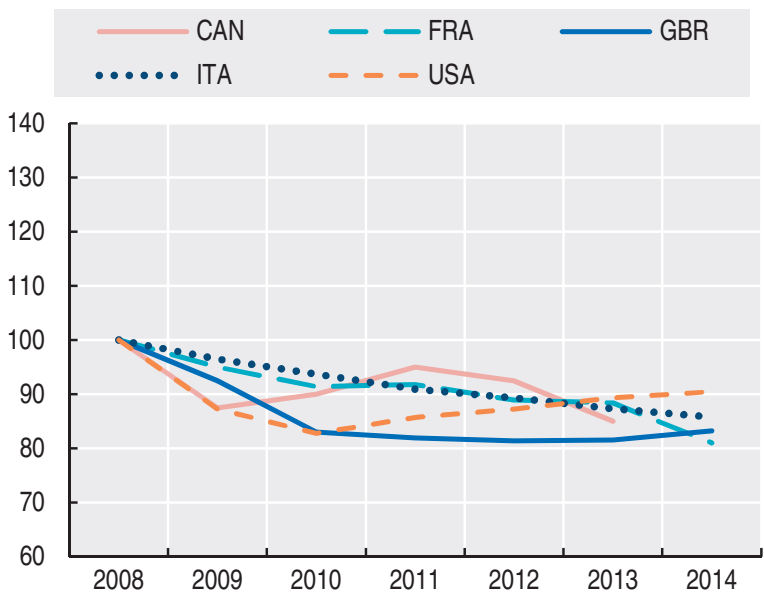

Services - Grandes entreprises
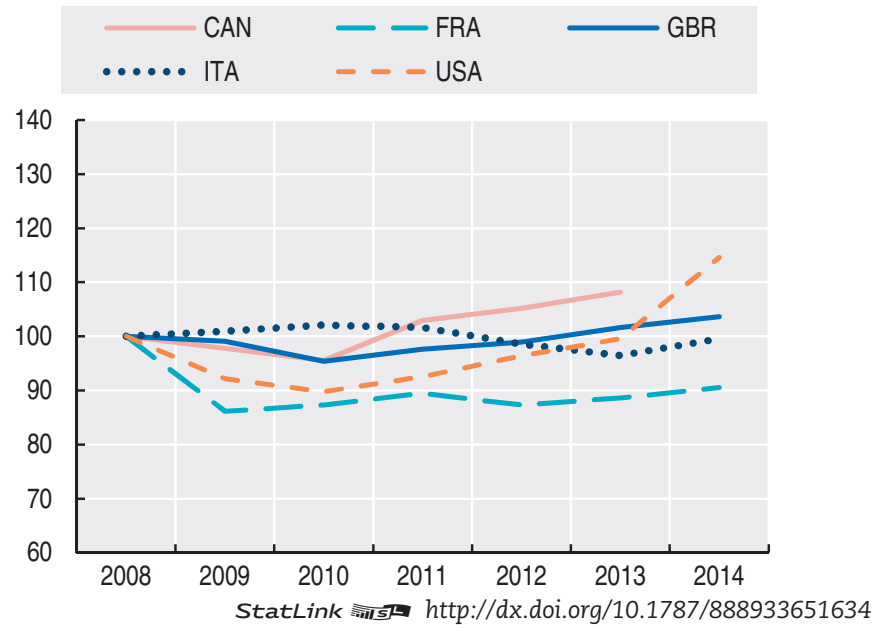
Graphique 2.2. Entreprises par taille, secteur marchand

En pourcentage de l'ensemble des entreprises, 2014 ou dernière année disponible

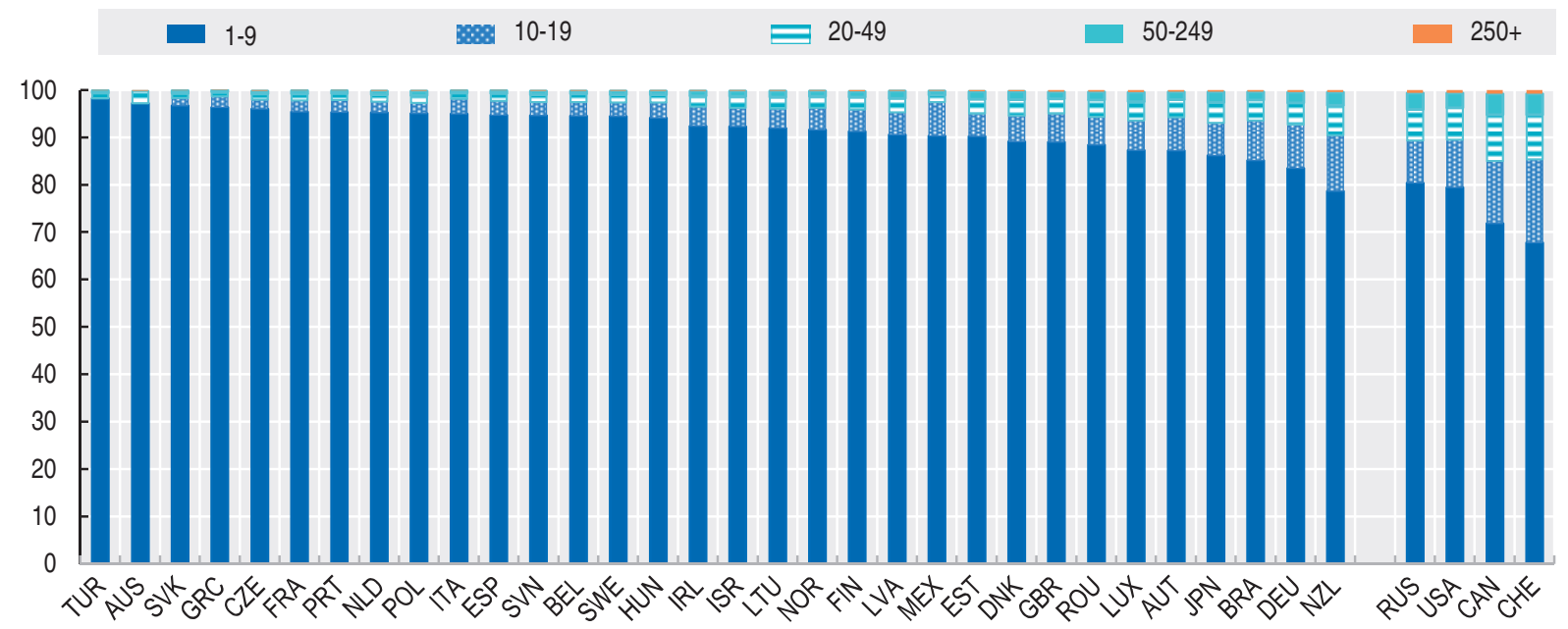

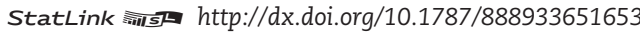

Tableau 2.1. Nombre d'entreprises par taille et principaux secteurs

2014, ou dernière année disponible

\begin{tabular}{|c|c|c|c|c|c|c|c|c|c|c|c|c|c|c|c|}
\hline \multirow{2}{*}{ Pays } & \multicolumn{5}{|c|}{ Activités de fabrication (10-33, CITI4) } & \multicolumn{5}{|c|}{ Services (45-82 hors 64-66, CITI4) } & \multicolumn{5}{|c|}{ Construction (41-43, CITI4) } \\
\hline & $1-9$ & $10-19$ & $20-49$ & $50-249$ & $250+$ & $1-9$ & $10-19$ & $20-49$ & $50-249$ & $250+$ & $1-9$ & $10-19$ & $20-49$ & $50-249$ & $250+$ \\
\hline Australie & 116,480 & & 6,403 & & 491 & $1,023,185$ & & 29,162 & & 1,899 & 337,841 & & 4,179 & & 166 \\
\hline Autriche & 18,576 & 2,842 & 2,182 & 1,456 & 468 & 228,685 & 15,980 & 7,586 & 3,070 & 483 & 27,984 & 3,593 & 1,985 & 597 & 68 \\
\hline Belgique & 29,944 & 2,331 & 1,992 & 1,169 & 311 & 425,095 & 12,262 & 6,321 & 2,256 & 470 & 101,414 & 2,695 & 1,362 & 476 & 51 \\
\hline Brésil & 218,056 & 52,029 & 36,051 & 17,029 & 3,830 & $2,436,010$ & 201,195 & 89,385 & 29,583 & 6,317 & 79,672 & 18,141 & 12,975 & 6,786 & 1,444 \\
\hline Canada & 27,887 & 8,202 & 7,842 & 5,545 & 1,426 & 442,857 & 84,182 & 58,994 & 26,516 & 4,232 & 114,195 & 14,314 & 8,578 & 3,605 & 554 \\
\hline $\begin{array}{l}\text { République } \\
\text { tchèque }\end{array}$ & 157,909 & 4,427 & 3,866 & 3,045 & 794 & 608,722 & 11,693 & 6,370 & 2,704 & 537 & 166,011 & 2,674 & 1,521 & 546 & 54 \\
\hline Danemark & 10,686 & 1,800 & 1,374 & 955 & 192 & 145,333 & 7,817 & 4,621 & 2,169 & 394 & 27,876 & 1,929 & 1,096 & 338 & 42 \\
\hline Estonie & 4,984 & 589 & 548 & 429 & 63 & 43,761 & 1,916 & 1,065 & 506 & 85 & 8,189 & 507 & 252 & 71 & 10 \\
\hline Finlande & 17,035 & 1,707 & 1,279 & 822 & 199 & 149,499 & 6,656 & 3,488 & 1,531 & 327 & 38,495 & 2,035 & 988 & 273 & 36 \\
\hline France & 205,876 & 12,761 & 9,554 & 5,545 & 1,357 & $2,194,656$ & 46,097 & 28,660 & 11,901 & 2,455 & 553,442 & 13,717 & 6,858 & 1,487 & 229 \\
\hline Allemagne & 138,436 & 37,010 & 16,420 & 16,484 & 4,252 & $1,650,651$ & 149,686 & 83,924 & 34,690 & 6,205 & 282,570 & 41,135 & 11,180 & 3,417 & 233 \\
\hline Grèce & 55,447 & 1,132 & 936 & 581 & 115 & 529,809 & 13,366 & 4,677 & 1,542 & 211 & 82,243 & 1,549 & 688 & 177 & 15 \\
\hline Hongrie & 40,097 & 3,131 & 2,360 & 1,623 & 403 & 382,982 & 11,125 & 4,869 & 2,045 & 352 & 53,212 & 2,275 & 979 & 279 & 20 \\
\hline Irlande & 12,503 & 823 & 664 & 494 & 144 & 153,628 & 7,838 & 4,142 & 1,876 & 273 & 46,180 & 731 & 333 & 89 & 16 \\
\hline Israël & 19,287 & 1,850 & 1,420 & 1,061 & 191 & 322,699 & 12,455 & 7,629 & 3,437 & 639 & 53,390 & 2,702 & 1,152 & 275 & 21 \\
\hline Italie & 328,486 & 39,402 & 18,988 & 8,349 & 1,197 & $2,649,255$ & 61,824 & 21,397 & 8,512 & 1,639 & 509,648 & 14,000 & 4,335 & 1,041 & 79 \\
\hline Japon & 315,669 & 42,791 & 34,305 & 21,591 & 3,576 & $1,845,690$ & 124,559 & 77,552 & 39,878 & 6,836 & 397,861 & 36,298 & 16,781 & 4,827 & 545 \\
\hline Corée & 328,505 & 33,847 & 23,963 & 10,155 & 701 & $2,321,477$ & 74,551 & 37,252 & 15,522 & 1,486 & 106,539 & 13,080 & 6,017 & 2,353 & 226 \\
\hline Lettonie & 7,859 & 756 & 655 & 476 & 59 & 73,262 & 3,186 & 1,727 & 749 & 117 & 8,030 & 687 & 488 & 203 & 16 \\
\hline Lituanie & 15,006 & 1,182 & 956 & 699 & 132 & 115,962 & 4,767 & 2,518 & 1,077 & 152 & 25,550 & 1,007 & 646 & 304 & 36 \\
\hline Luxembourg & 503 & 101 & 105 & 78 & 25 & 24,083 & 1,421 & 771 & 380 & 97 & 2,592 & 443 & 352 & 137 & 18 \\
\hline Mexique & 430,971 & 39,242 & 8,338 & 7,431 & 3,548 & $2,509,306$ & 186,689 & 32,713 & 16,808 & 2,087 & 4,904 & 6,429 & 3,370 & 2,069 & 291 \\
\hline Pays-Bas & 53,105 & 3,382 & 2,657 & 1,924 & 326 & 790,223 & 18,447 & 10,867 & 5,427 & 1,042 & 149,560 & 2,791 & 1,601 & 683 & 113 \\
\hline Nouvelle-Zélande & 7,956 & 1,851 & 1,287 & 594 & 132 & 57,351 & 8,754 & 4,440 & 1,983 & 309 & 17,205 & 1,821 & 846 & 273 & 21 \\
\hline Norvège & 13,898 & 1,308 & 1,040 & 646 & 124 & 193,988 & 8,975 & 4,265 & 1,832 & 402 & 50,538 & 2,767 & 1,402 & 399 & 44 \\
\hline Pologne & 157,056 & 8,580 & 7,327 & 6,131 & 1,545 & $1,066,190$ & 20,258 & 12,038 & 6,286 & 1,226 & 221,638 & 4,438 & 2,927 & 1,340 & 154 \\
\hline Portugal & 54,420 & 5,527 & 3,943 & 2,061 & 250 & 610,235 & 11,571 & 5,287 & 2,137 & 413 & 73,245 & 2,845 & 1,271 & 433 & 50 \\
\hline Roumanie & 34,577 & 5,174 & 4,438 & 3,118 & 784 & 320,262 & 17,272 & 8,964 & 3,506 & 609 & 40,669 & 3,805 & 2,296 & 956 & 87 \\
\hline $\begin{array}{l}\text { Fédération de } \\
\text { Russie }\end{array}$ & 146,355 & 24,594 & 23,509 & 16,061 & 4,428 & $1,238,672$ & 123,612 & 82,829 & 38,918 & 4,264 & 196,983 & 26,146 & 21,049 & 10,156 & 1,105 \\
\hline $\begin{array}{l}\text { République } \\
\text { slovaque }\end{array}$ & 60,348 & 2,201 & 1,187 & 964 & 275 & 239,723 & 3,590 & 2,079 & 1,015 & 203 & 82,645 & 733 & 377 & 158 & 14 \\
\hline Slovénie & 16,452 & 947 & 578 & 477 & 107 & 86,592 & 2,023 & 1,040 & 450 & 80 & 17,098 & 679 & 262 & 84 & 10 \\
\hline Espagne & 140,164 & 12,397 & 9,176 & 4,124 & 728 & $1,733,714$ & 49,478 & 23,401 & 8,929 & 1,794 & 335,541 & 6,947 & 3,319 & 893 & 121 \\
\hline Suède & 47,482 & 2,752 & 2,072 & 1,284 & 306 & 473,822 & 12,574 & 7,144 & 3,206 & 567 & 91,020 & 3,370 & 1,755 & 501 & 48 \\
\hline Suisse & 11,476 & 4,432 & 3,072 & 1,933 & 389 & 73,093 & 16,782 & 8,015 & 3,573 & 664 & 14,118 & 4,257 & 2,425 & 798 & 63 \\
\hline Turquie & 607,180 & - & 18,076 & 9,384 & 1,784 & $3,815,186$ & - & 25,727 & 9,960 & 2,064 & 312,450 & - & 10,048 & 4,553 & 442 \\
\hline Royaume-Uni & 95,804 & 13,081 & 9,485 & 6,220 & 1,377 & $1,274,346$ & 86,371 & 42,248 & 18,640 & 4,299 & 252,747 & 11,003 & 4,873 & 1,832 & 315 \\
\hline États-Unis & 228,477 & 46,273 & 37,114 & 22,893 & 5,543 & $2,742,717$ & 344,321 & 223,482 & 100,313 & 19,732 & 511,722 & 53,162 & 31,594 & 12,375 & 1,353 \\
\hline
\end{tabular}


Graphique 2.3. Nombre d'entreprises et PIB

2014, ou dernière année disponible

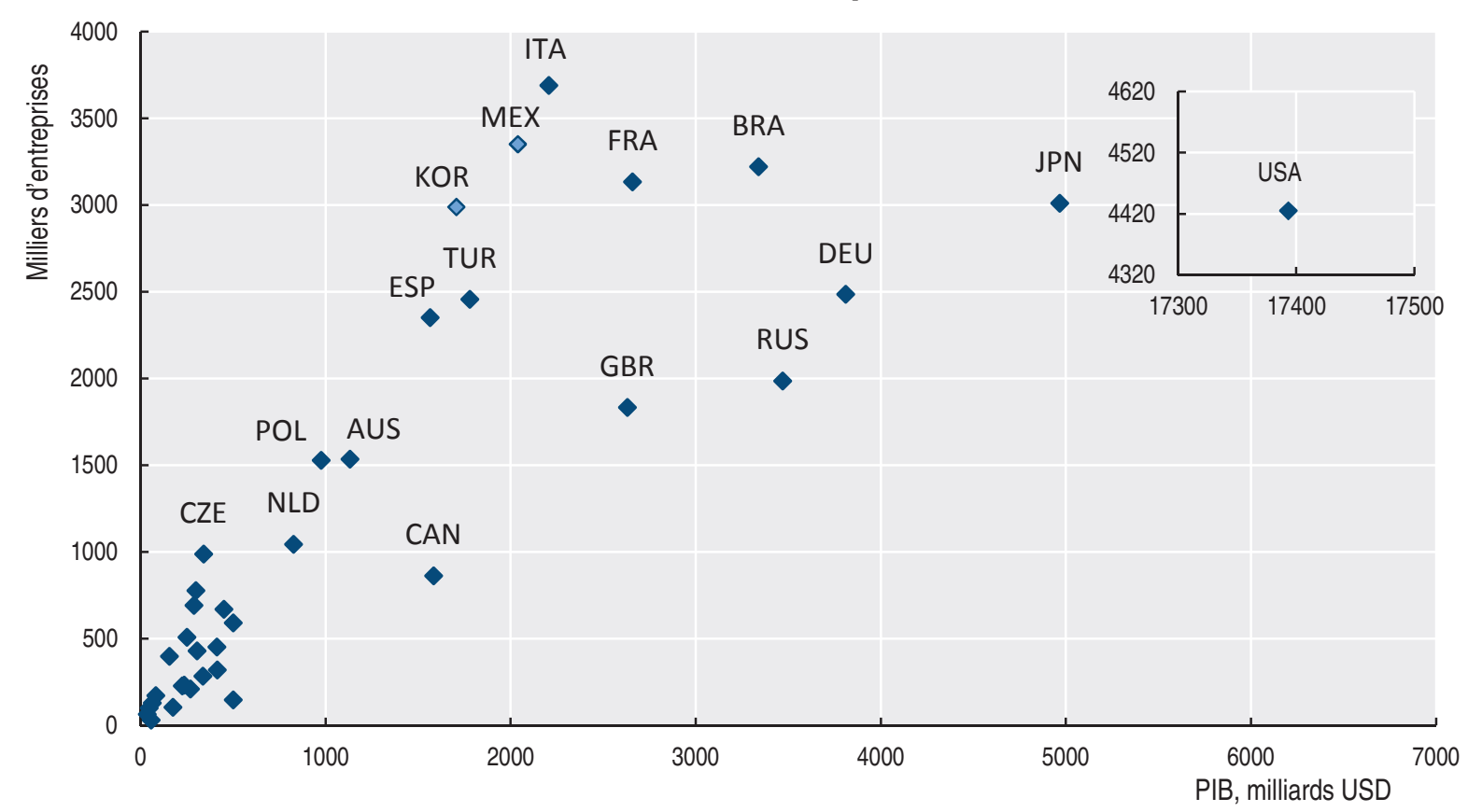

StatLink -insts http://dx.doi.org/10.1787/888933651691

Graphique 2.4. Part des entreprises sans salarié, secteur marchand

En pourcentage de la population totale des entreprises, 2014, ou dernière année disponible

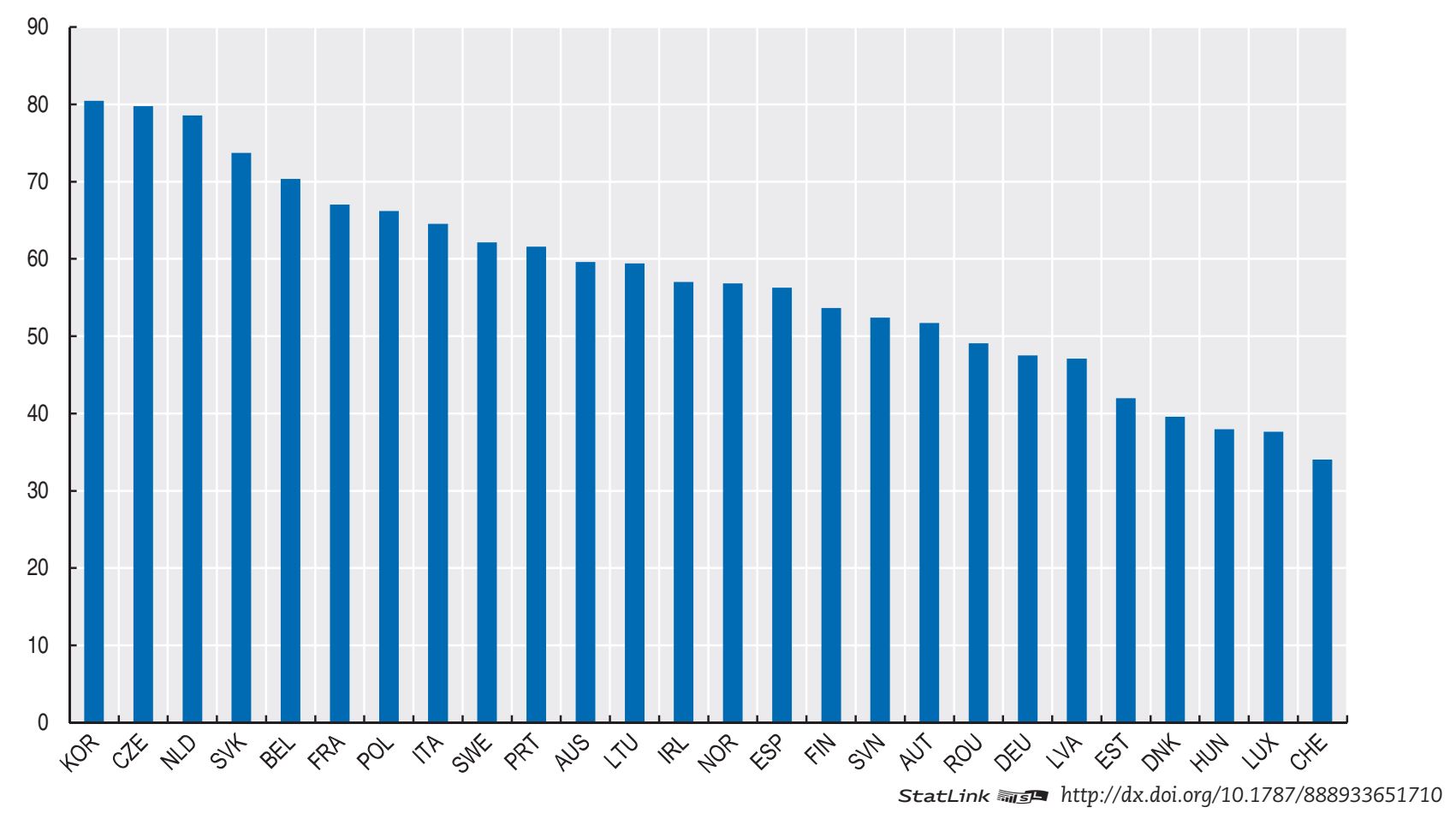


Graphique 2.5. Nombre de PME par activité économique

En pourcentage du nombre total de PME, 2014 ou dernière année disponible

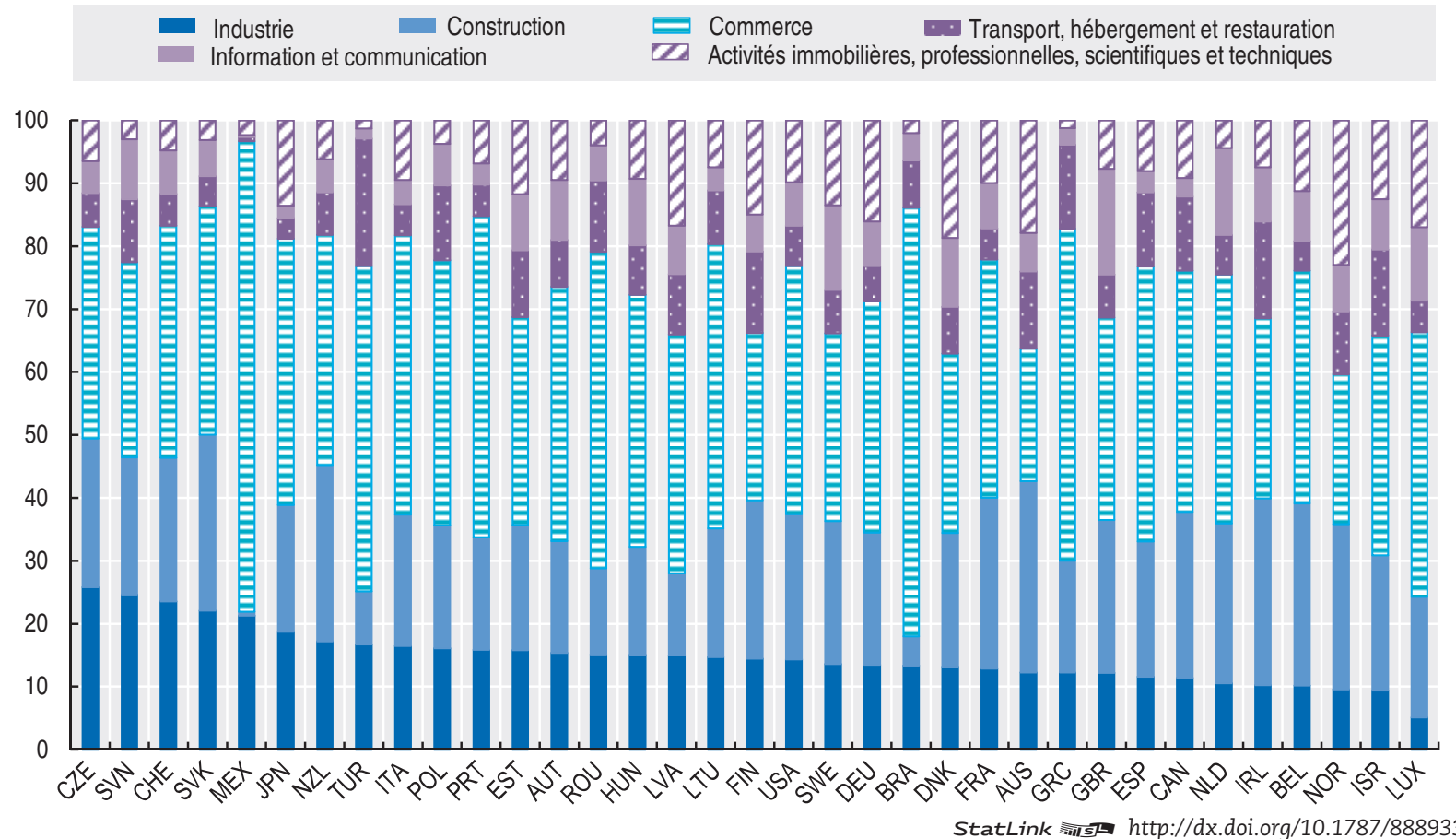

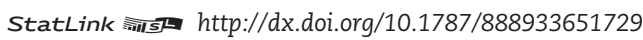

Graphique 2.6. Évolution du nombre d'entreprises par taille, secteur marchand

Pourcentage moyen d'évolution annuelle entre 2008 et 2014

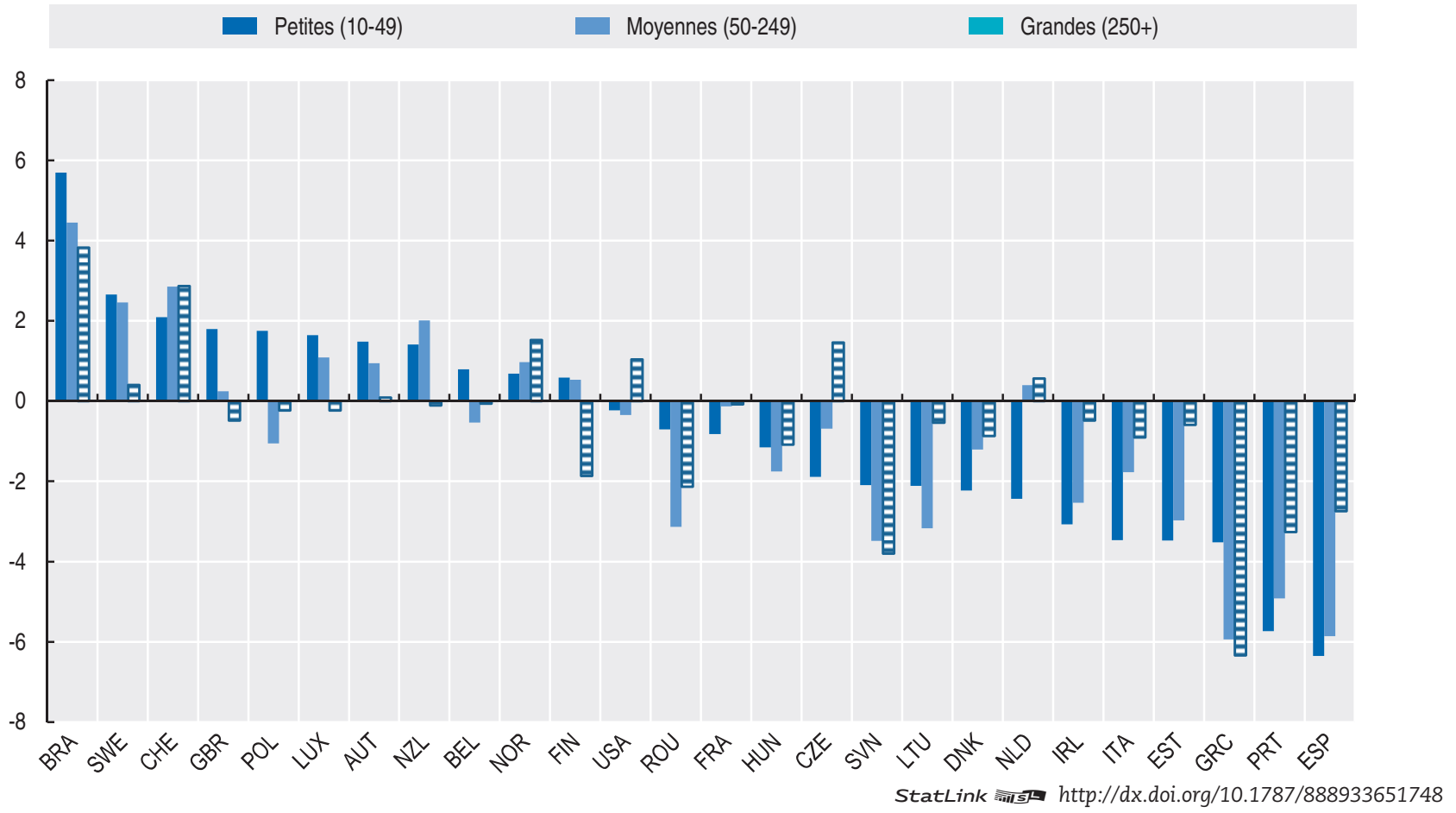


Graphique 2.7. Évolution du nombre d'entreprises, par taille et principaux secteurs Pourcentage moyen d'évolution annuelle entre 2008 et 2014

Activités de fabrication

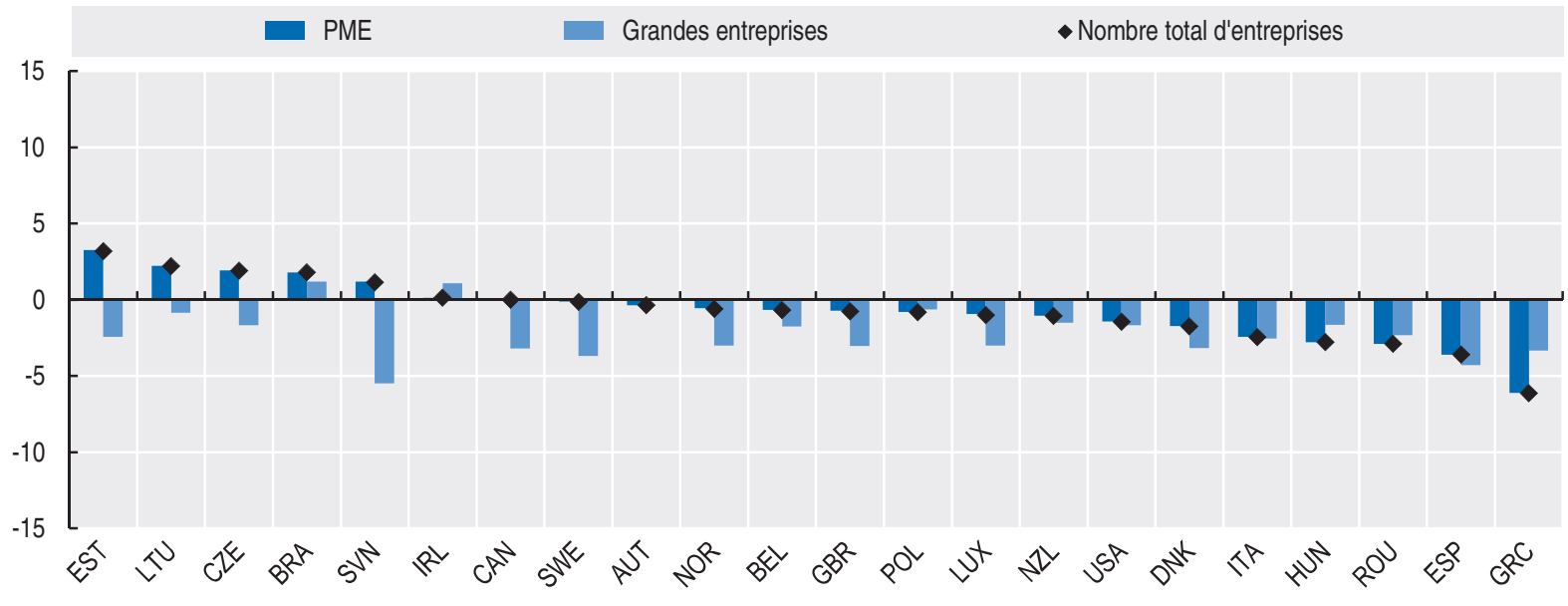

Services

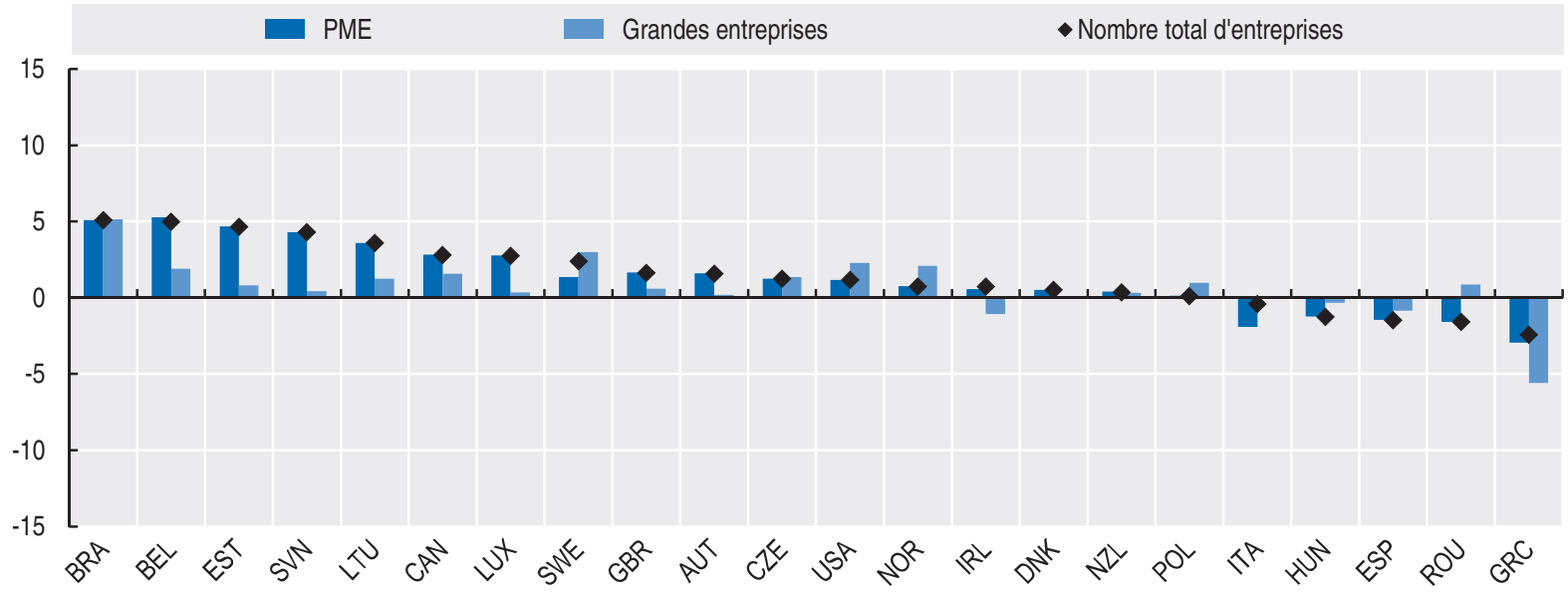

Construction

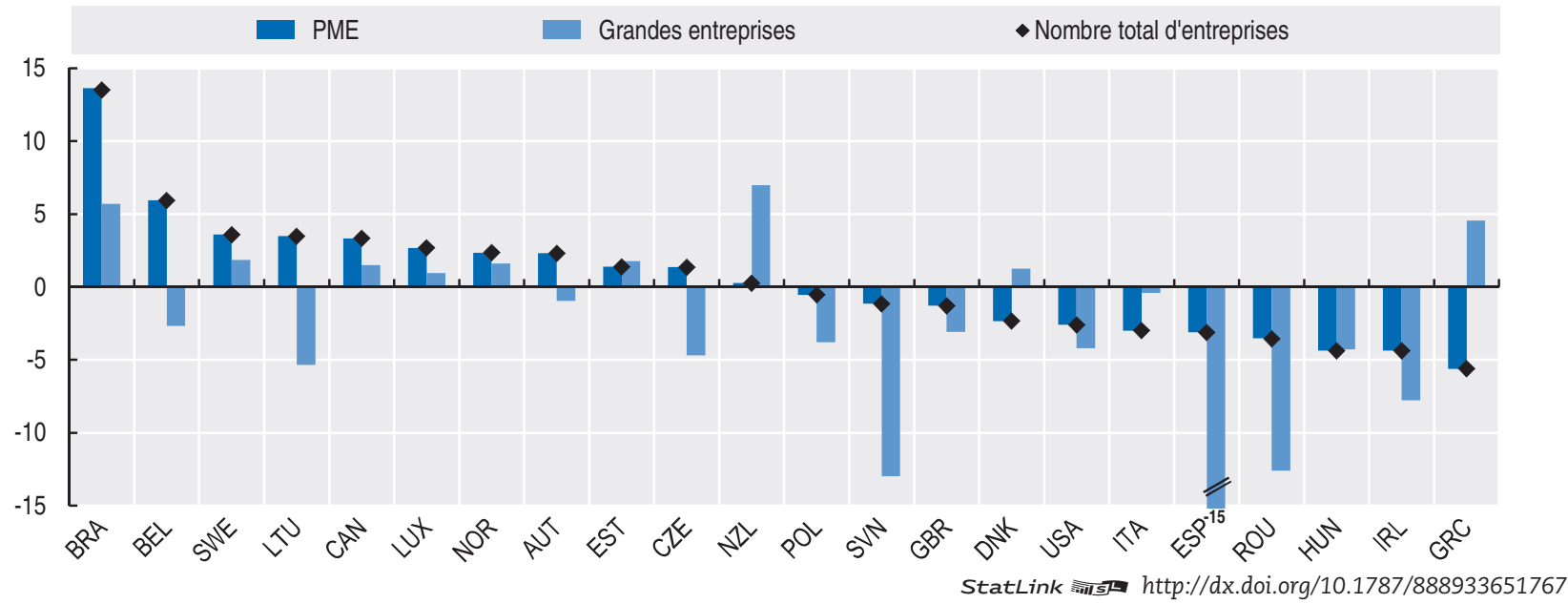




\section{À savoir}

- Bien que les grandes entreprises représentent moins de $1 \%$ de la population totale des entreprises, elles génèrent une fraction beaucoup plus importante de l'emploi - entre $47 \%$ des personnes occupées travaillant dans le secteur marchand au Royaume-Uni et $12 \%$ en Grèce. En moyenne, dans les pays de l'OCDE, les grandes entreprises sont à l'origine d'environ $40 \%$ de l'emploi manufacturier total, et d'environ $25 \%$ de l'emploi dans le secteur des services.

- Entre 2008 et 2014, l'emploi dans le secteur de la fabrication a diminué dans tous les pays de l'OCDE sauf deux : le Luxembourg et l'Allemagne, ce qui reflète principalement la baisse du nombre d'entreprises, qu'il s'agisse de PME ou de grandes entreprises. En 2014, les niveaux d'emploi dans les pays les plus durement touchés par la crise restaient inférieurs à ceux de 2008, le recul affectant surtout les PME. De même, dans la plupart des économies où l'emploi dépassait le pic d'avant la crise, les PME étaient les principaux moteurs de croissance. Aux États-Unis, ce sont les grandes entreprises du secteur des services qui ont tiré la croissance de l'emploi après la crise.

- Au sein du secteur de la fabrication, la croissance de l'emploi dans les grandes entreprises de la zone euro, qui ont été moins durement touchées par la crise que les PME, est restée plus rapide que dans ces dernières, alors que l'inverse est vrai aux États-Unis.

\section{Pertinence}

Les PME sont d'importants moteurs de croissance de l'emploi, mais peuvent aussi être plus vulnérables aux retournements de conjoncture. Une meilleure compréhension de la répartition de l'emploi peut apporter un éclairage utile sur la résilience sousjacente et sur la sécurité de l'emploi, ainsi que sur le potentiel de croissance de l'emploi. Conjuguées aux données relatives aux rémunérations moyennes, qui montrent généralement que les rémunérations sont d'autant plus faibles que l'entreprise est petite, les données relatives à la répartition peuvent fournir une analyse plus fine des inégalités de revenu.

\section{Définitions}

Le nombre de personnes occupées correspond au nombre total de personnes qui ont travaillé pour l'unité d'observation pendant l'année de référence (y compris les propriétaires exploitants, les associés exerçant une activité régulière dans l'unité en question, et les travailleurs familiaux non rémunérés). Sont exclus les administrateurs de sociétés de capitaux et les membres des comités d'actionnaires qui ne perçoivent que des jetons de présence pour les réunions auxquelles ils assistent, la main-d'œuvre mise à la disposition de l'unité par d'autres unités à ses frais, les personnes chargées de l'entretien et des réparations de l'unité pour le compte d'autres unités, ainsi que les travailleurs à domicile. Sont également exclues les personnes en congé de durée indéterminée, accomplissant leur service militaire ou ne touchant pas d'autre rémunération de l'entreprise qu'une pension.

L'évolution globale du nombre de personnes occupées se décompose en quatre facteurs : la variation du nombre de PME et de grandes entreprises, et la variation de la taille moyenne des PME et des grandes entreprises.
La contribution de la variation du nombre de PME équivaut au produit de la variation du nombre de PME entre 2008 et 2014 et de la taille moyenne des PME en 2008 La contribution de la variation de la taille moyenne des PME est le produit de la variation de la taille moyenne des PME entre 2008 et 2014 et du nombre de PME en 2014. Ces deux contributions sont calculées de façon analogue pour les grandes entreprises. La part relative de chaque contribution est l'expression de la valeur absolue de cette contribution en pourcentage de la variation totale du nombre de personnes occupées (autrement dit, la somme de la valeur absolue de toutes les contributions). L'effectif employé moyen par taille d'entreprise est le nombre de personnes occupées dans une classe de taille, divisé par le nombre d'entreprises enregistrées dans cette classe de taille dans un secteur économique donné.

Informations sur les données concernant Israël : http:// dx.doi.org/10.1787/888932315602.

\section{Comparabilité}

L'unité statistique sur laquelle reposent les informations présentées est l'entreprise pour tous les pays sauf la Corée et le Mexique, qui utilisent l'établissement. Les données relatives à l'emploi dans tous les pays désignent le nombre de personnes occupées, sauf pour : la Suisse, où les données excluent l'emploi dans les entreprises comptant moins de 3 salariés ; le Canada, la Corée, les États-Unis, la Fédération de Russie, Israël et le Japon, où les données se rapportent aux salariés. Pour l'ensemble du secteur marchand, le nombre d'entreprises sans salarié s'élève approximativement à 1.7 million au Canada, 15.3 millions aux États-Unis et 2.5 millions dans la Fédération de Russie.

Pour le Royaume-Uni, les données présentées n'incluent pas les quelque 2.6 millions de petites entreprises non enregistrées, c'est-à-dire des entreprises n'atteignant pas le seuil prévu pour le régime de la taxe sur la valeur ajoutée et/ou celui de la retenue de l'impôt à la source (pour les entreprises employant des salariés). Certains pays utilisent des conventions différentes : la classe « 1-9 " correspond à «1-10" pour le Mexique et à «1-19 " pour l'Australie, le Canada et la Turquie ; la classe " 10-19 » correspond à « 11-50 " pour le Mexique ; la classe « 50-249 " à « 20-199 " pour l'Australie, à « 51-250 " pour le Mexique, et à " 50-299 " pour le Canada, la Corée et le Japon ; enfin, la classe "250+ " correspond à « 200+ " pour l'Australie, «300+ " pour le Canada, la Corée et le Japon, et à « 251+ » pour le Mexique. Il convient d'interpréter avec précaution les variations dans le temps, car les données ne suivent pas des cohortes d'entreprises. Des grandes entreprises dont la taille décroît peuvent se retrouver classées comme PME, et de façon analogue, la croissance des PME peut avoir pour conséquence leur classement parmi les grandes entreprises.

\section{Source}

OCDE, Statistiques structurelles et démographiques des entreprises (SDBS) (base de données), http://dx.doi.org/10.1787/sdbs-data-fr.

\section{Pour en savoir plus}

OCDE (2017), Small, Medium, Strong. Trends in SME Performance and Business Conditions, Éditions OCDE, Paris, http://dx.doi. org/10.1787/9789264275683-en. 
Graphique 2.8. Emploi par taille d'entreprise, zone euro et États-Unis

Nombre d'emplois, $2008=100$

\section{Secteur marchand}

Zone euro

$-1-9-10-49-250+$

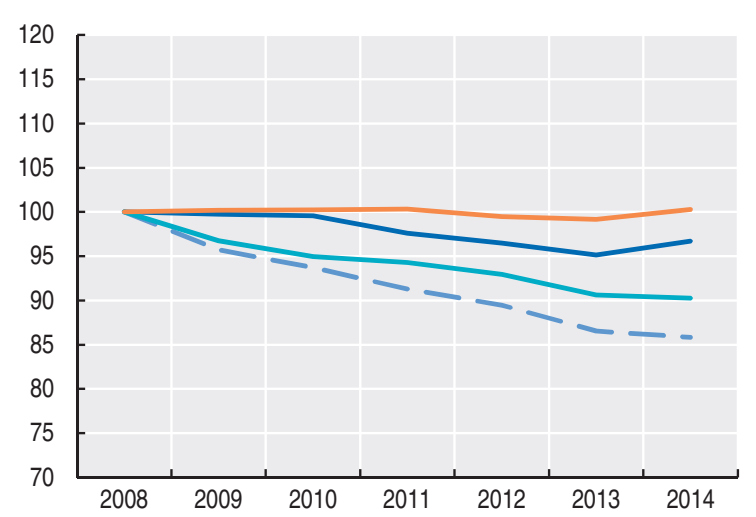

États-Unis
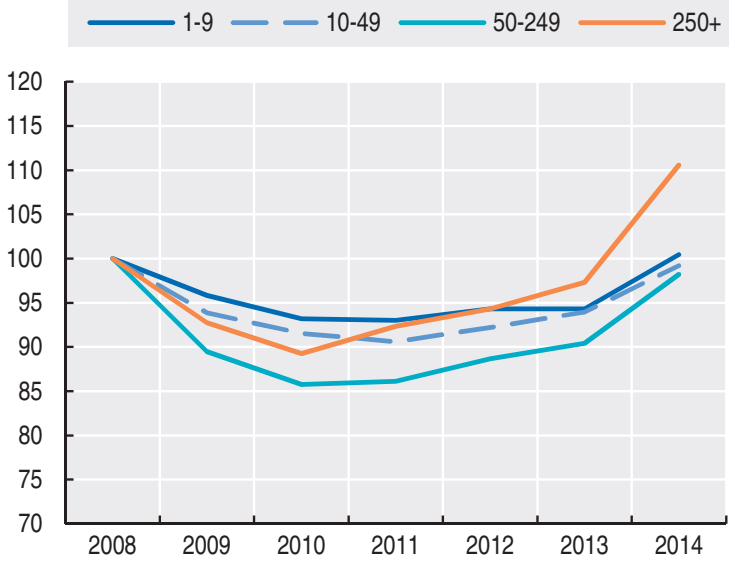

Activités de fabrication

Zone euro

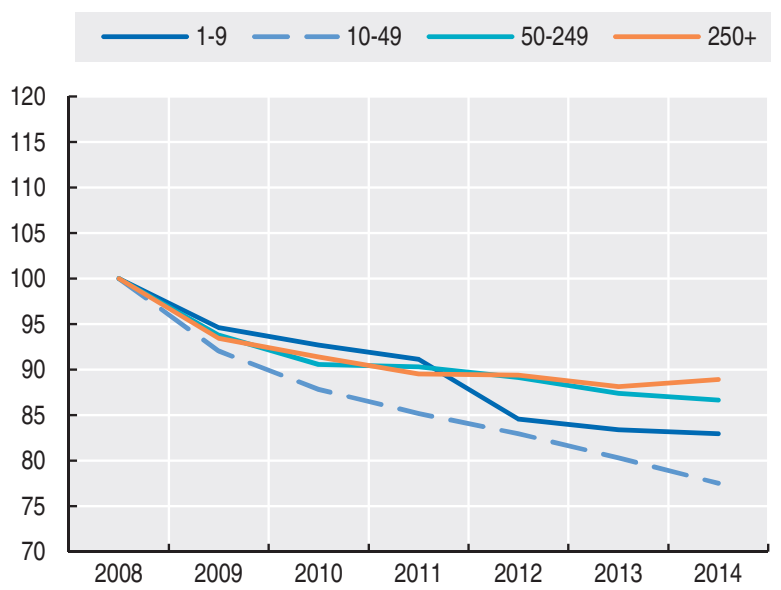

États-Unis
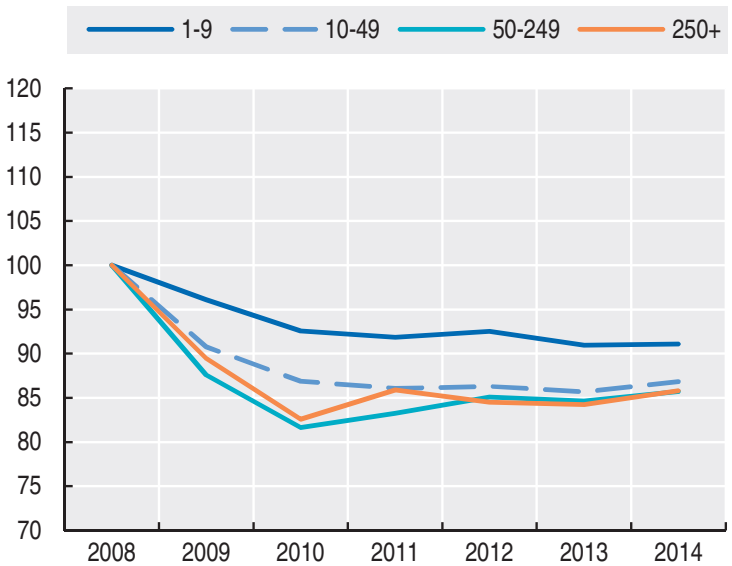

Services

Zone euro
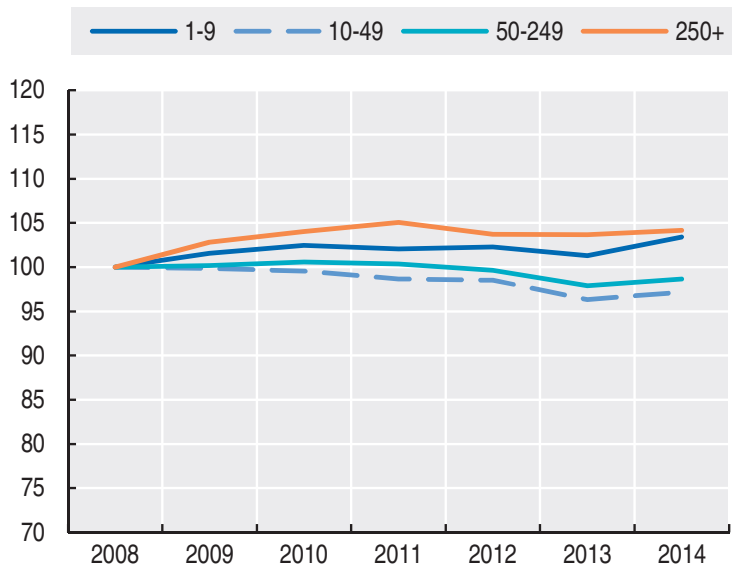

États-Unis
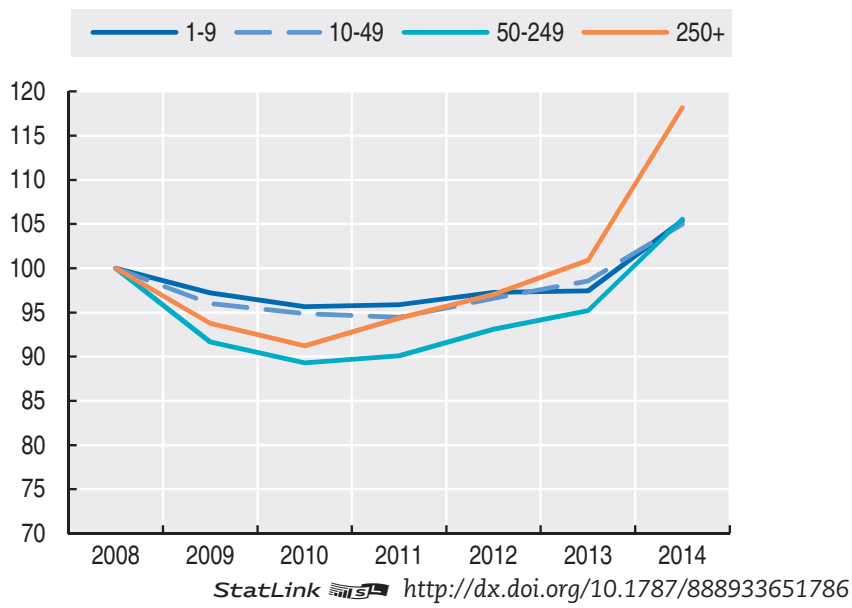
Graphique 2.9. Effectif employé par taille d'entreprise, secteur marchand

En pourcentage de l'emploi total, 2014 ou dernière année disponible

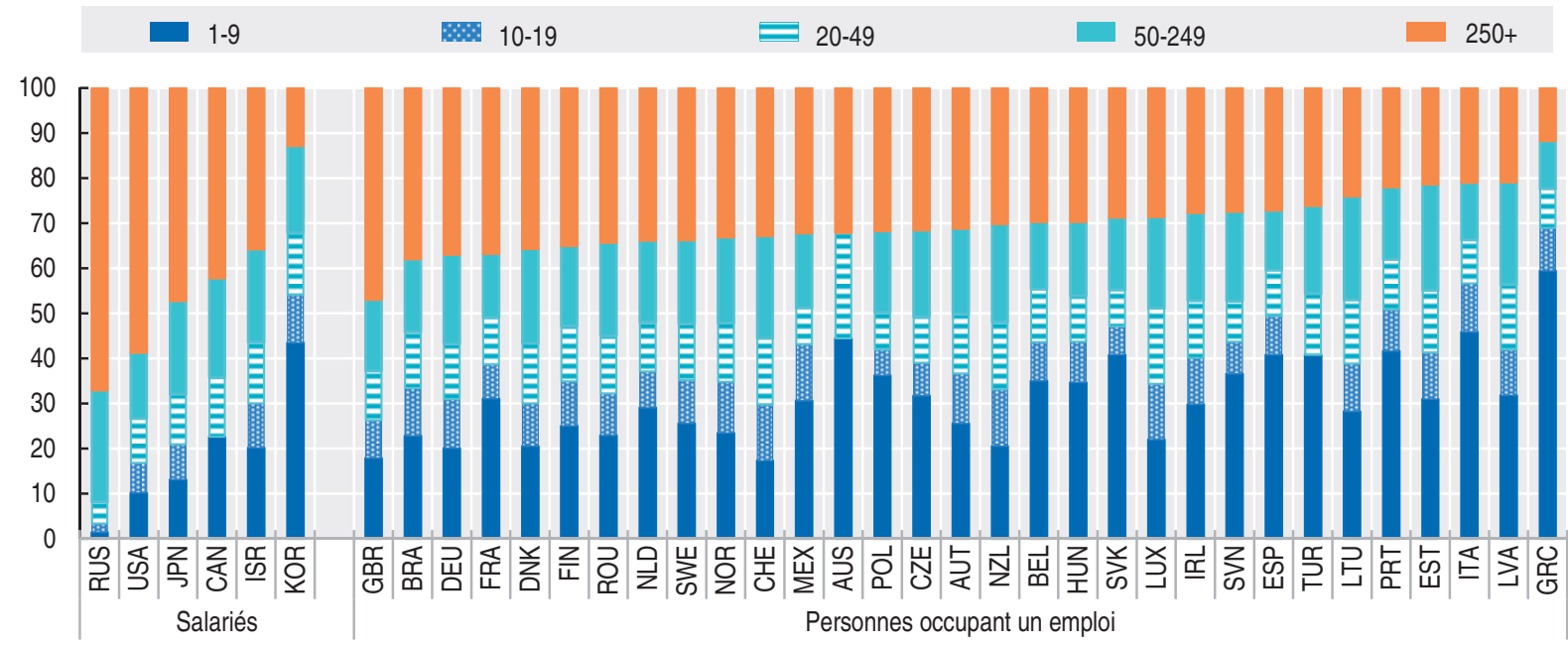

StatLink नilst $h t t p: / / d x . d o i . o r g / 10.1787 / 888933651805$

Tableau 2.2. Effectif employé par taille d'entreprise, secteur marchand

2014 ou dernière année disponible

\begin{tabular}{|c|c|c|c|c|c|c|}
\hline Pays & $1-9$ & $10-19$ & $20-49$ & $50-249$ & $250+$ & Total \\
\hline Australie & $3,526,000$ & & $1,859,000$ & & $2,541,000$ & $7,926,000$ \\
\hline Autriche & 692,580 & 302,047 & 355,028 & 519,675 & 843,661 & $2,712,991$ \\
\hline Belgique & 946,348 & 232,801 & 316,460 & 404,696 & 801,748 & $2,702,053$ \\
\hline Brésil & $7,787,469$ & $3,661,851$ & $4,258,283$ & $5,527,970$ & $12,954,846$ & $34,190,419$ \\
\hline Canada & $2,381,568$ & & $1,382,103$ & $2,320,477$ & $4,429,641$ & $10,513,789$ \\
\hline République tchèque & $1,108,872$ & 254,141 & 356,862 & 671,844 & $1,100,426$ & $3,492,145$ \\
\hline Danemark & 334,699 & 156,685 & 216,595 & 344,423 & 581,782 & $1,634,184$ \\
\hline Estonie & 124,549 & 41,632 & 55,263 & 94,830 & 85,736 & 402,010 \\
\hline Finlande & 366,248 & 145,587 & 179,851 & 260,668 & 511,812 & $1,464,166$ \\
\hline France & $4,799,169$ & $1,176,958$ & $1,602,109$ & $2,206,025$ & $5,672,971$ & $15,457,232$ \\
\hline Allemagne & $5,558,583$ & $3,043,919$ & $3,377,932$ & $5,516,994$ & $10,241,981$ & $27,739,409$ \\
\hline Grèce & $1,276,724$ & 206,287 & 187,379 & 228,794 & 249,678 & $2,148,862$ \\
\hline Hongrie & 852,339 & 223,372 & 250,067 & 405,303 & 728,848 & $2,459,929$ \\
\hline Irlande & 363,428 & 126,923 & 154,828 & 239,185 & 337,178 & $1,221,542$ \\
\hline Israël & 458,069 & 227,705 & 309,210 & 469,332 & 813,522 & $2,277,838$ \\
\hline Italie & $6,469,991$ & $1,531,284$ & $1,356,694$ & $1,799,667$ & $2,951,263$ & $14,108,899$ \\
\hline Japon & $4,607,136$ & $2,789,088$ & $3,907,695$ & $7,297,740$ & $16,626,035$ & $35,227,694$ \\
\hline Corée & $6,528,613$ & $1,624,470$ & $2,055,787$ & $2,903,018$ & $1,924,597$ & $15,036,485$ \\
\hline Lettonie & 196,115 & 63,122 & 88,957 & 140,615 & 128,651 & 617,460 \\
\hline Lituanie & 250,168 & 93,916 & 125,271 & 203,336 & 212,164 & 884,855 \\
\hline Luxembourg & 45,263 & 25,632 & 34,569 & 41,649 & 58,856 & 205,969 \\
\hline Mexique & $5,670,630$ & $2,328,081$ & $1,499,174$ & $3,077,857$ & $5,942,343$ & $18,518,085$ \\
\hline Pays-Bas & $1,537,522$ & 424,692 & 579,738 & 956,911 & $1,787,609$ & $5,286,472$ \\
\hline Nouvelle-Zélande & 268,655 & 167,209 & 194,173 & 287,310 & 394,720 & $1,312,067$ \\
\hline Norvège & 367,681 & 177,626 & 204,653 & 299,511 & 517,052 & $1,566,523$ \\
\hline Pologne & $3,031,711$ & 486,859 & 690,145 & $1,517,269$ & $2,655,280$ & $8,381,264$ \\
\hline Portugal & $1,200,901$ & 266,943 & 320,986 & 462,714 & 632,845 & $2,884,389$ \\
\hline Roumanie & 877,379 & 356,873 & 486,767 & 793,666 & $1,308,907$ & $3,823,592$ \\
\hline Fédération de Russie & 255,837 & 363,909 & 892,153 & $4,588,166$ & $12,366,162$ & $18,466,227$ \\
\hline République slovaque & 579,089 & 89,648 & 114,156 & 229,617 & 405,531 & $1,418,041$ \\
\hline Slovénie & 203,000 & 39,808 & 49,745 & 110,653 & 151,632 & 554,838 \\
\hline Espagne & $4,325,165$ & 916,784 & $1,078,404$ & $1,407,623$ & $2,875,335$ & $10,603,311$ \\
\hline Suède & 768,683 & 288,919 & 376,499 & 559,952 & $1,011,281$ & $3,005,334$ \\
\hline Suisse & 481,764 & 346,787 & 409,103 & 634,678 & 911,757 & $2,784,089$ \\
\hline Turquie & $5,097,885$ & & $1,689,941$ & $2,439,017$ & $3,255,113$ & $12,481,956$ \\
\hline Royaume-Uni & $3,301,459$ & $1,556,195$ & $2,011,219$ & $2,920,352$ & $8,654,750$ & $18,443,975$ \\
\hline États-Unis & $9,245,888$ & $6,048,538$ & $8,918,826$ & $13,305,796$ & $53,250,192$ & $90,769,240$ \\
\hline
\end{tabular}

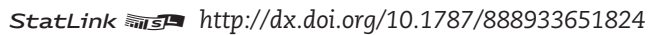


Graphique 2.10. Effectif employé par taille d'entreprise, principaux secteurs

En pourcentage de l'ensemble des personnes occupées par secteur, 2014 ou dernière année disponible

Activités de fabrication

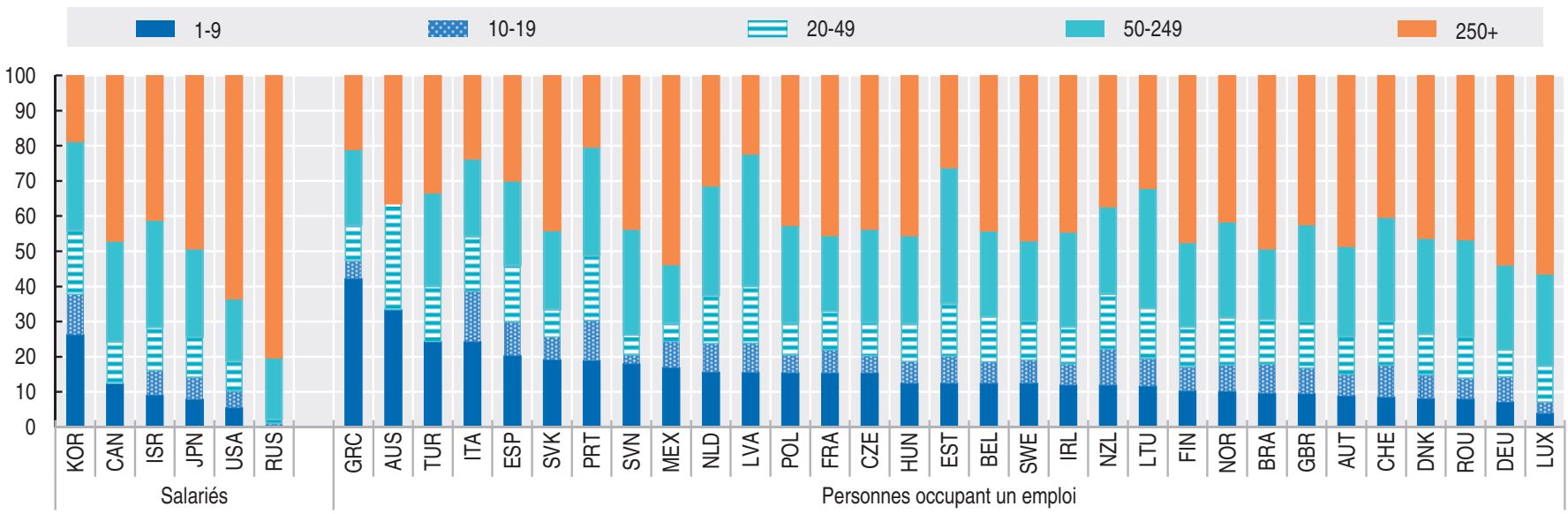

Services

$\begin{array}{llll}1-9 & 10-19 & \equiv 20-49 & 50-249\end{array}$

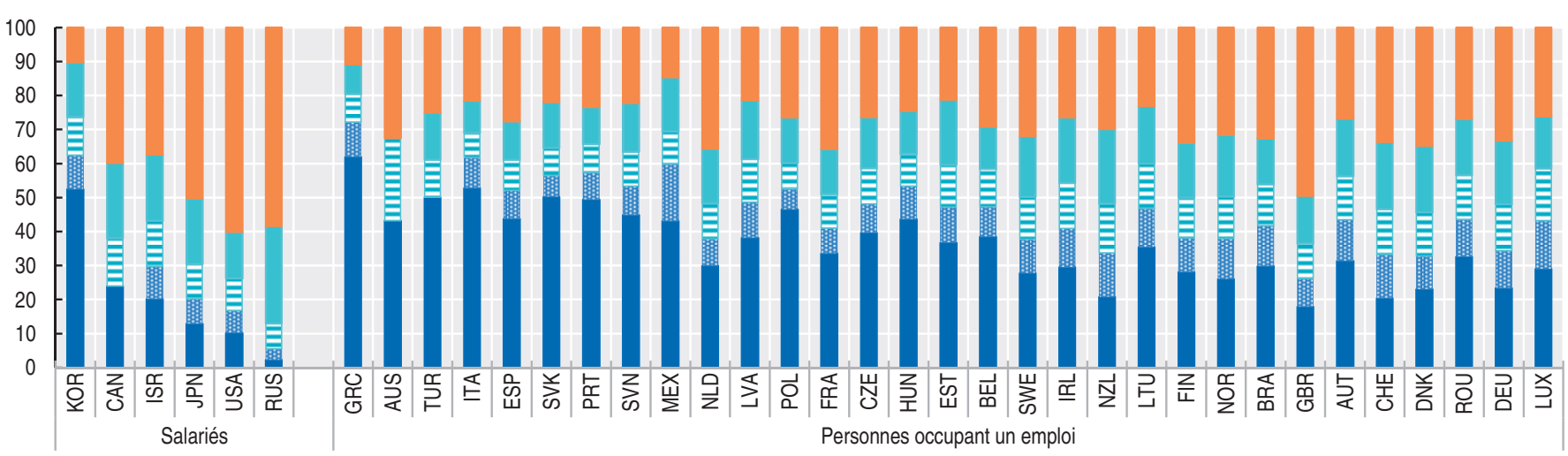

Construction

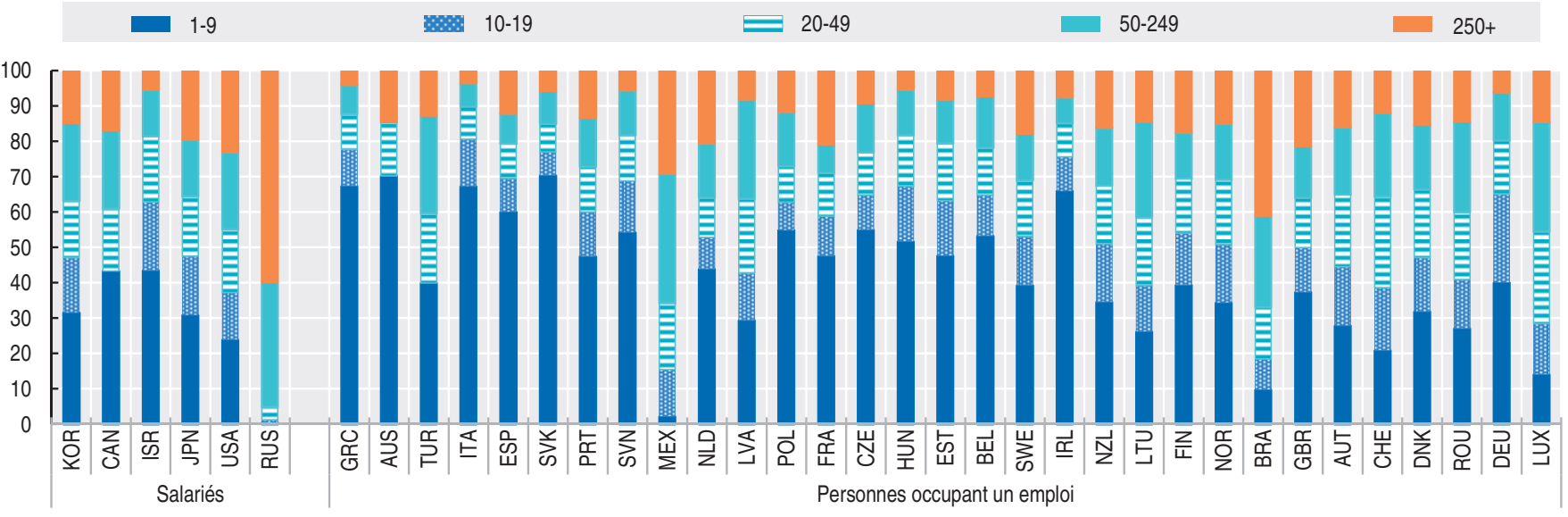

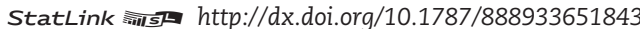


Graphique 2.11. Emploi dans les PME et les grandes entreprises par activité économique Pourcentage de l'emploi dans le secteur marchand par taille d'entreprise, 2014 ou dernière année disponible

PME

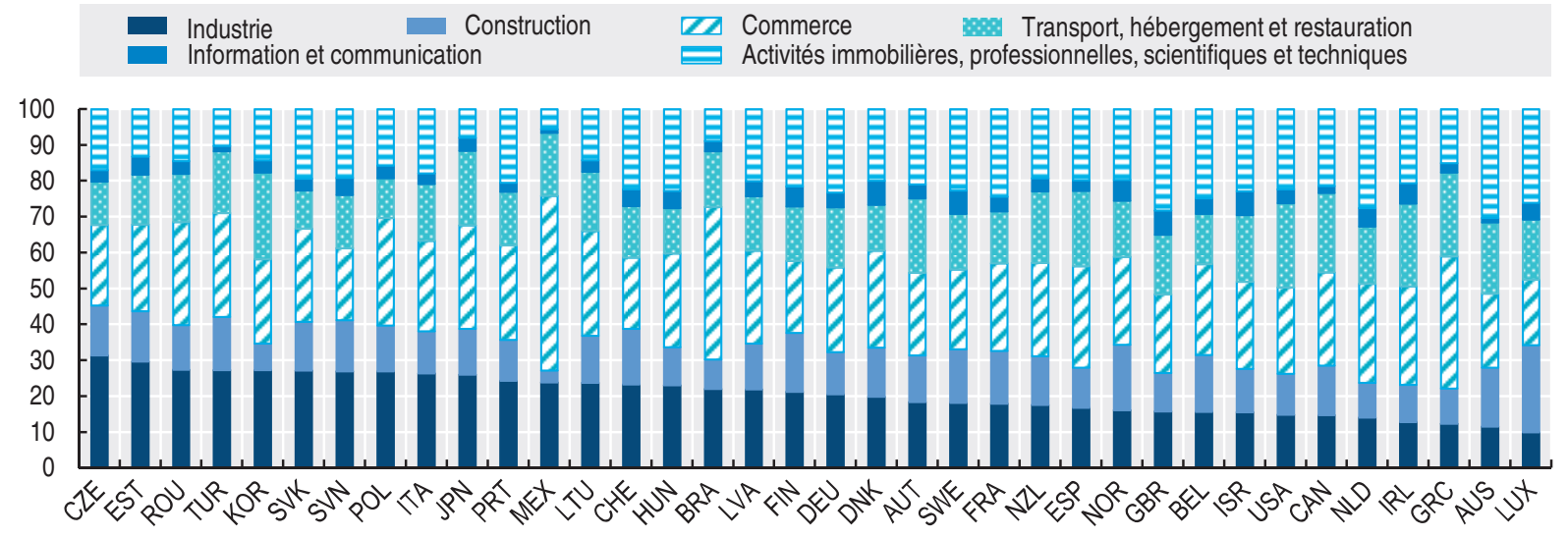

Grandes entreprises

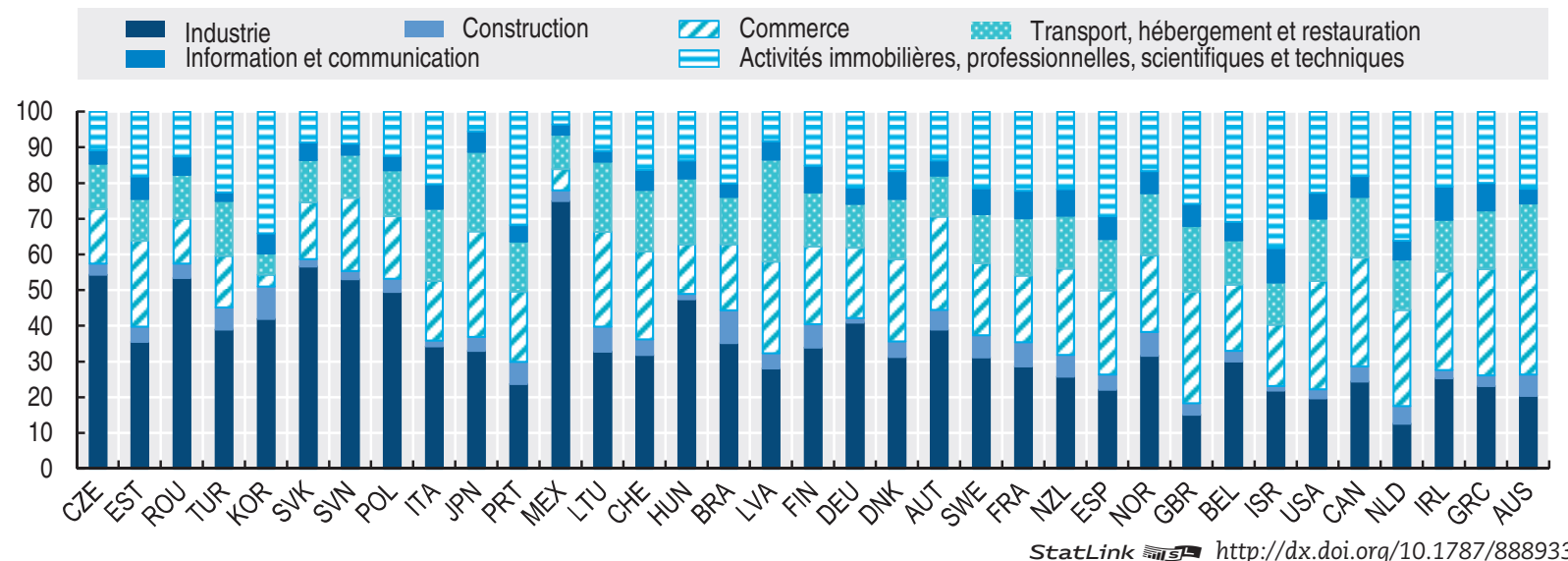

StatLink त्ताIs http://dx.doi.org/10.1787/888933651862

Graphique 2.12. Variation de l'effectif, secteur marchand

Contributions et pourcentage de variation entre 2008 et 2014

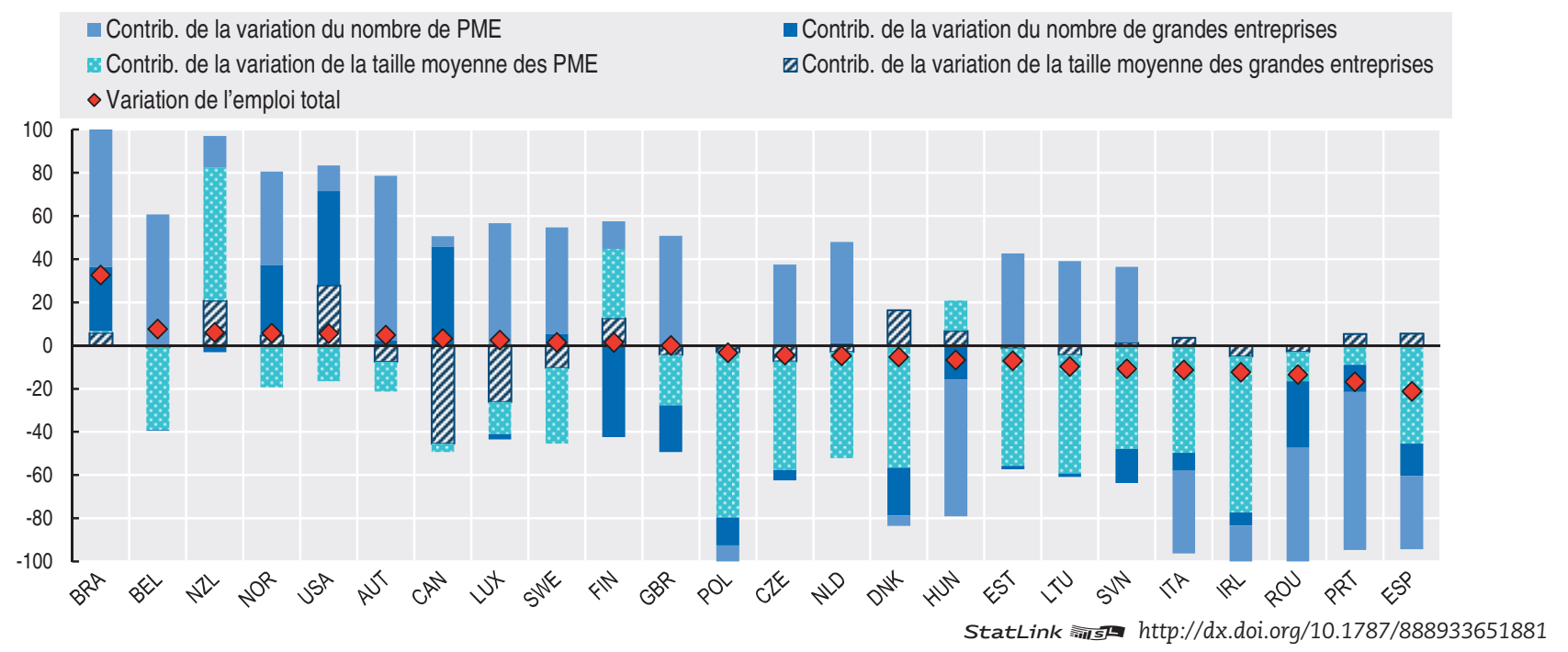


Graphique 2.13. Variation de l'effectif, principaux secteurs

Contributions et pourcentage de variation entre 2008 et 2014

\section{Activités de fabrication}

- Contrib. de la variation du nombre de PME

- Contrib. de la variation de la taille moyenne des PME

- Variation de l'emploi total
- Contrib. de la variation du nombre de grandes entreprises

चContrib. de la variation de la taille moyenne des grandes entreprises

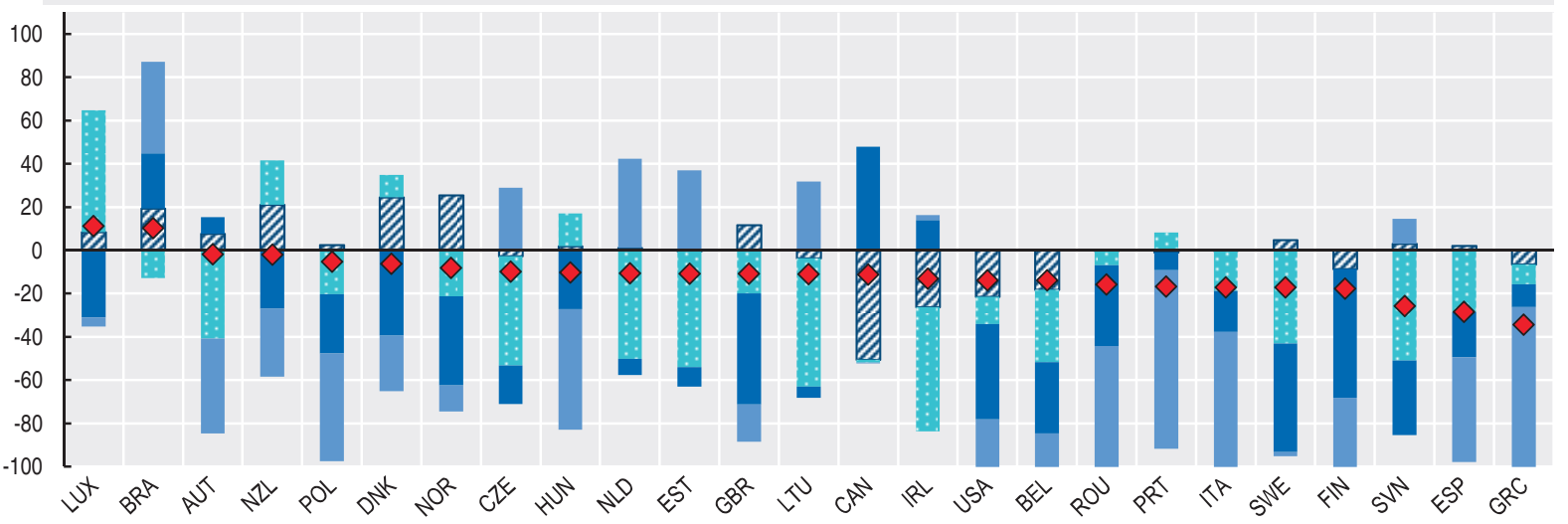

\section{Services}

- Contrib. de la variation du nombre de PME

- Contrib. de la variation de la taille moyenne des PME

- Contrib. de la variation du nombre de grandes entreprises

$\diamond$ Variation de l'emploi total

øContrib. de la variation de la taille moyenne des grandes entreprises

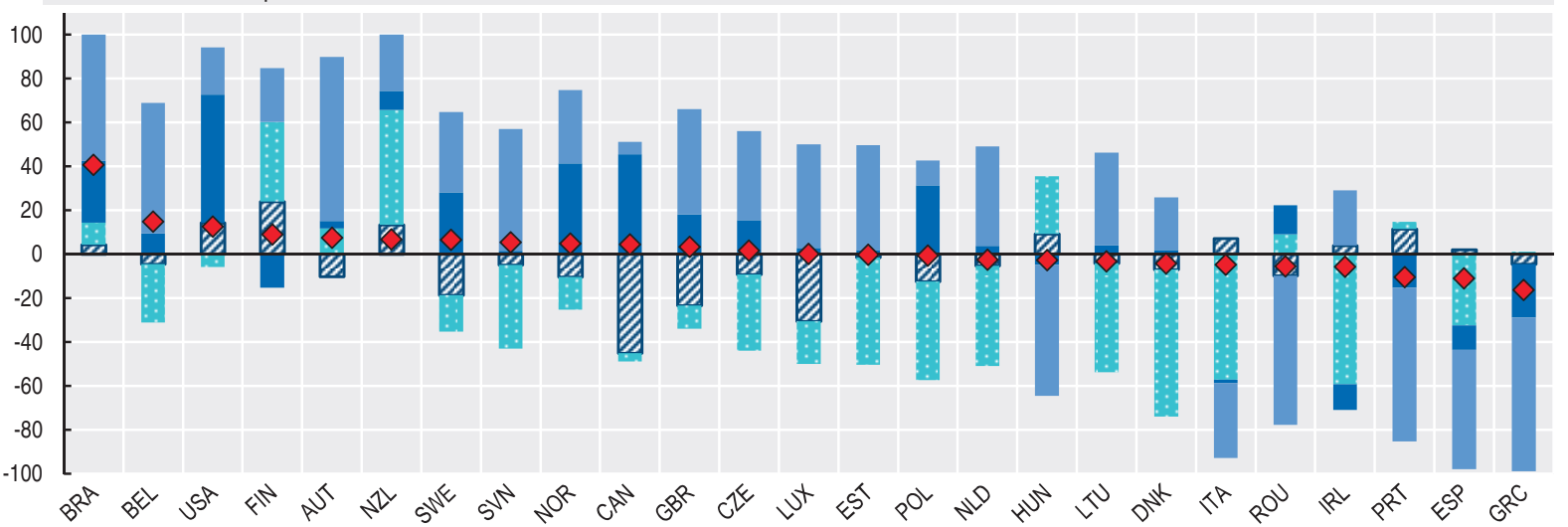

\section{Construction}

- Contrib. de la variation du nombre de PME

- Contrib. de la variation de la taille moyenne des PME

$\diamond$ Variation de l'emploi total
- Contrib. de la variation du nombre de grandes entreprises चContrib. de la variation de la taille moyenne des grandes entreprises

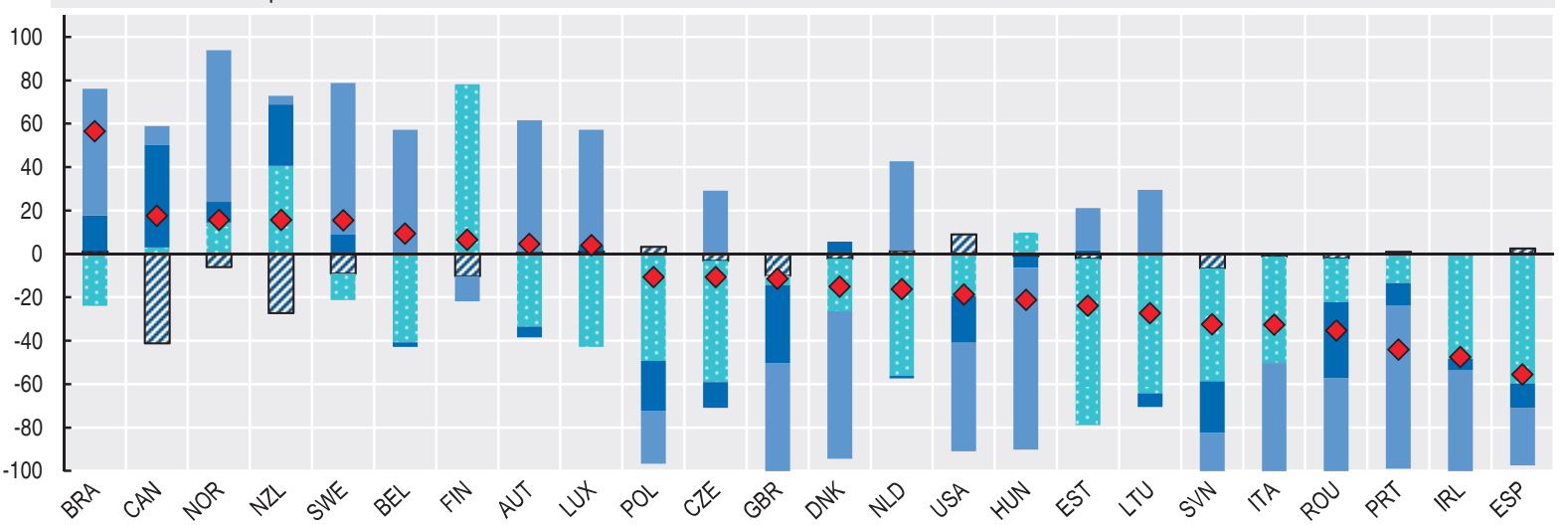




\section{Emploi par taille d'entreprise}

Tableau 2.3. Emploi moyen par principaux secteurs et taille d'entreprise

Emploi par entreprise, nombre de personnes, 2014 ou dernière année disponible

\begin{tabular}{|c|c|c|c|c|c|c|c|c|c|c|c|c|}
\hline \multirow{2}{*}{ Pays } & \multicolumn{4}{|c|}{ Activités de fabrication } & \multicolumn{4}{|c|}{ Services } & \multicolumn{4}{|c|}{ Construction } \\
\hline & $1-9$ & $10-49$ & $50-249$ & $250+$ & $1-9$ & $10-49$ & $50-249$ & $250+$ & $1-9$ & $10-49$ & $50-249$ & $250+$ \\
\hline AUT & 3 & 21 & 110 & 644 & 2 & 18 & 97 & 969 & 3 & 19 & 92 & 680 \\
\hline BEL & 2 & 22 & 106 & 716 & 2 & 20 & 101 & 1,142 & 2 & 20 & 99 & 464 \\
\hline BRA & 4 & 20 & 100 & 1,090 & 3 & 19 & 102 & 1,156 & 4 & 21 & 109 & 811 \\
\hline CHE & 5 & 20 & 105 & 701 & 5 & 18 & 95 & 876 & 5 & 21 & 98 & 625 \\
\hline CZE & 1 & 22 & 108 & 674 & 1 & 18 & 99 & 872 & 1 & 19 & 94 & 634 \\
\hline DEU & 4 & 20 & 107 & 920 & 3 & 19 & 96 & 953 & 3 & 17 & 87 & 585 \\
\hline DNK & 3 & 21 & 103 & 869 & 2 & 20 & 96 & 949 & 2 & 19 & 92 & 617 \\
\hline ESP & 2 & 21 & 101 & 707 & 2 & 18 & 98 & 1,180 & 2 & 19 & 95 & 995 \\
\hline EST & 3 & 22 & 97 & 446 & 2 & 19 & 91 & 607 & 3 & 18 & 76 & 356 \\
\hline FIN & 2 & 21 & 102 & 826 & 2 & 19 & 95 & 931 & 2 & 19 & 90 & 926 \\
\hline FRA & 2 & 24 & 117 & 1,010 & 2 & 24 & 115 & 1,492 & 2 & 21 & 99 & 1,652 \\
\hline GBR & 2 & 23 & 111 & 764 & 2 & 21 & 107 & 1,642 & 2 & 22 & 107 & 907 \\
\hline GRC & 2 & 21 & 106 & 513 & 2 & 17 & 94 & 852 & 2 & 18 & 91 & 539 \\
\hline HUN & 2 & 21 & 105 & 764 & 2 & 18 & 95 & 1,056 & 2 & 18 & 90 & 531 \\
\hline IRL & 2 & 21 & 104 & 584 & 2 & 19 & 95 & 892 & 1 & 18 & 82 & 473 \\
\hline ISR & 2 & 21 & 101 & 757 & 1 & 20 & 96 & 988 & 2 & 18 & 86 & 453 \\
\hline ITA & 3 & 19 & 97 & 717 & 2 & 17 & 98 & 1,154 & 2 & 17 & 87 & 607 \\
\hline JPN & 2 & 21 & 107 & 1,443 & 2 & 20 & 107 & 1,314 & 2 & 18 & 95 & 1,045 \\
\hline KOR & 3 & 35 & 99 & 1,048 & 3 & 27 & 102 & 725 & 2 & 28 & 101 & 668 \\
\hline LTU & 2 & 22 & 101 & 501 & 2 & 18 & 87 & 840 & 1 & 20 & 92 & 415 \\
\hline LUX & 3 & 16 & 111 & 741 & 2 & 16 & 53 & 353 & 2 & 21 & 94 & 337 \\
\hline LVA & 2 & 21 & 96 & 458 & 2 & 19 & 94 & 743 & 2 & 20 & 94 & 346 \\
\hline MEX & 2 & 14 & 115 & 766 & 2 & 13 & 102 & 755 & 3 & 19 & 101 & 570 \\
\hline NLD & 2 & 24 & 110 & 645 & 2 & 26 & 123 & 1,413 & 1 & 20 & 96 & 784 \\
\hline NOR & 2 & 21 & 101 & 794 & 1 & 18 & 99 & 793 & 2 & 19 & 89 & 776 \\
\hline NZL & 4 & 20 & 100 & 711 & 3 & 19 & 101 & 825 & 3 & 18 & 91 & 891 \\
\hline POL & 2 & 22 & 110 & 667 & 2 & 20 & 100 & 1,014 & 2 & 20 & 95 & 632 \\
\hline PRT & 2 & 21 & 98 & 527 & 2 & 18 & 96 & 1,072 & 2 & 18 & 94 & 786 \\
\hline ROU & 3 & 22 & 105 & 700 & 2 & 19 & 98 & 914 & 2 & 20 & 98 & 603 \\
\hline SVK & 1 & 20 & 109 & 748 & 2 & 19 & 101 & 825 & 1 & 19 & 86 & 608 \\
\hline SVN & 2 & 20 & 108 & 701 & 2 & 18 & 97 & 813 & 2 & 18 & 87 & 345 \\
\hline SWE & 2 & 23 & 112 & 953 & 1 & 22 & 109 & 1,099 & 2 & 21 & 96 & 1,352 \\
\hline TUR & & & 103 & 675 & 1 & & 100 & 865 & & & 95 & 453 \\
\hline USA & 3 & 21 & 101 & 1,501 & 3 & 20 & 96 & 2,150 & 2 & 20 & 93 & 903 \\
\hline
\end{tabular}


Graphique 2.14. Évolution de la taille moyenne des PME et des grandes entreprises $2008=100$
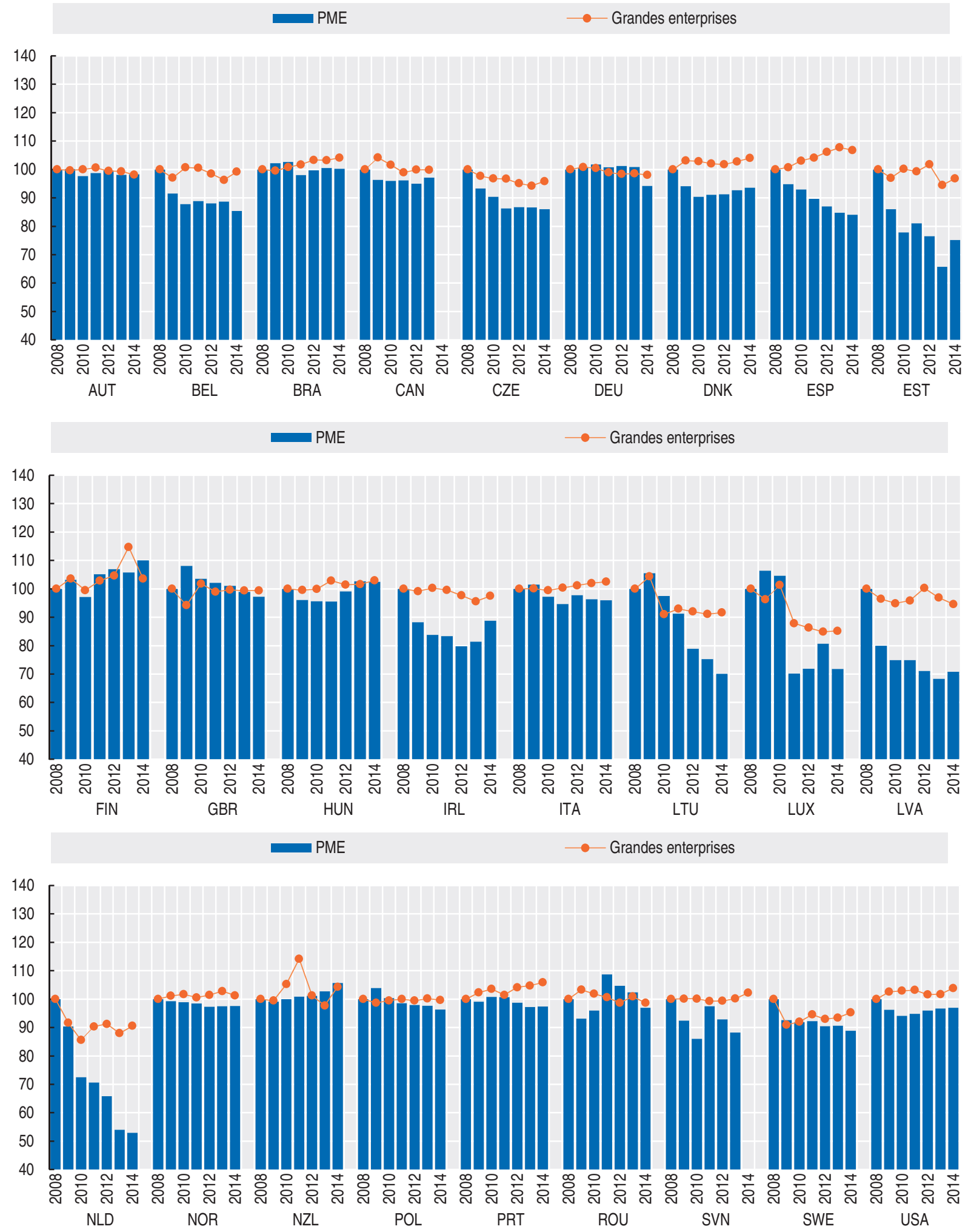

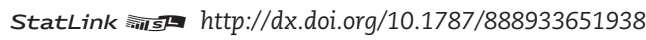




\section{À savoir}

- Dans la plupart des pays, les grandes entreprises qui emploient plus de 250 personnes produisent une part considérable de la valeur ajoutée du secteur marchand, alors qu'elles représentent moins de $1 \%$ de l'ensemble des entreprises. La proportion de valeur ajoutée créée par les grandes entreprises varie considérablement d'un pays à l'autre, traduisant l'importance économique et la structure de la population d'entreprises : d'environ $15 \%$ au Luxembourg à $32 \%$ en Italie, et plus de $60 \%$ au Mexique.

- Les grandes entreprises industrielles dominent le paysage des entreprises dans de nombreux pays. Dans près de la moitié des économies de l'OCDE, le secteur industriel représente plus de la moitié de la valeur ajoutée totale générée par les grandes entreprises du secteur marchand ; ce pourcentage va de $26 \%$ au Royaume-Uni à $85 \%$ au Mexique.

- Les PME se classent en tête du secteur des services dans presque tous les pays, où elles génèrent $60 \%$ ou plus du total de la valeur ajoutée. Les grandes entreprises, quant à elles, pèsent lourd dans la création de valeur ajoutée du secteur de la fabrication dans la plupart des pays, ce qui reflète en partie l'augmentation des rendements d'échelle induite par une production à plus forte intensité capitalistique. Toutefois, dans les États baltes et dans les pays d'Europe du Sud, les PME se taillent la part du lion dans le secteur de la fabrication, bien que la contribution des PME de grande taille (50 à 429 salariés) y soit bien plus importante que dans d'autres pays.

- Entre 2008 et 2014, la part relative des PME et des grandes entreprises dans la valeur ajoutée totale créée par le secteur de la fabrication est restée stable dans pratiquement tous les pays, à l'exception de l'Irlande.

\section{Pertinence}

Les performances entrepreneuriales et en matière de productivité varient amplement d'un pays à l'autre. Ces différences trouvent en partie leur explication dans l'hétérogénéité des entreprises. Les entreprises de grande taille, par exemple, ont généralement une productivité supérieure à celle des petites entreprises, et si les entreprises nouvellement créées sont souvent les moteurs de l'innovation,

\section{Définitions}

La valeur ajoutée correspond à la différence entre la production et la consommation intermédiaire, la consommation intermédiaire totale étant valorisée aux prix d'acquisition. Les mesures de la production utilisées ci-dessous diffèrent d'un pays à un autre et sont évaluées aux prix de base ou aux coûts des facteurs. Les mesures aux coûts des facteurs n'incluent pas les autres taxes et subventions à la production définies dans le Système de comptabilité nationale de 2008.

Les données de cette section présentent la valeur ajoutée dans chaque classe de taille (définie en fonction du nombre de personnes occupées), en pourcentage de la valeur ajoutée de l'ensemble des entreprises.

Informations sur les données concernant Israël : http:// dx.doi.org/10.1787/888932315602. de nombreuses microentreprises ont cependant un potentiel de croissance limité. La ventilation des données sur la valeur ajoutée en fonction de la taille de l'entreprise apporte par conséquent un éclairage important sur les facteurs structurels qui favorisent la croissance, l'emploi et la valeur entrepreneuriale, mais alimente aussi les préoccupations concernant le ralentissement de la diffusion de la productivité et le découplage entre productivité et salaires.

\section{Comparabilité}

Les données correspondent à la valeur ajoutée au coût des facteurs dans les pays de l'UE et aux prix de base pour les autres pays ; elles portent sur l'économie marchande, hors intermédiation financière.

Compte tenu des pratiques différentes des pays en matière de collecte de données, la ventilation par classe de taille utilisée, à savoir 1-9, 10-19, 20-49, 50-249 et 250+, offre une comparabilité optimale. Certains pays utilisent des conventions différentes : pour le Japon, « 50-249 » correspond à « 50+ "; pour le Mexique, « 1-9 » correspond à « 1-10 ", « 10-19 " correspond à « 11-20 », « 20-49 » correspond à « 21-50 ", « 50-249 » correspond à « 51-250 » et « 250+ » correspond à « 251+ "; pour la Turquie, « 1-9 » correspond à « 1-19".

L'unité statistique utilisée pour le Corée, le Mexique et les ÉtatsUnis n'est pas l'entreprise, mais l'établissement. Les données concernant Israël et les États-Unis n'incluent pas la valeur ajoutée créée par les entreprises sans salarié. Les données relatives au secteur de la fabrication en Corée n'incluent pas les établissements qui emploient 10 salariés ou moins. Les données pour la Suisse excluent les informations sur les entreprises de moins de trois salariés.

On observe une rupture de séries dans les données en 2013 pour la Finlande et le Portugal et en 2014 pour la France. Pour le Royaume-Uni, les données présentées n'incluent pas les quelque 2.6 millions de petites entreprises non enregistrées, c'est-à-dire des entreprises n'atteignant pas le seuil prévu pour le régime de la taxe sur la valeur ajoutée et/ou celui de la retenue de l'impôt à la source (pour les entreprises employant des salariés).

Il convient d'interpréter avec précaution les variations dans le temps, car les données ne suivent pas des cohortes d'entreprises. Des grandes entreprises dont la taille décroît peuvent se retrouver classées comme PME, et de façon analogue, la croissance des PME peut avoir pour conséquence leur classement parmi les grandes entreprises.

\section{Source}

OCDE, Statistiques structurelles et démographiques des entreprises (SDBS) (base de données), http://dx.doi.org/10.1787/sdbs-data-fr.

\section{Pour en savoir plus}

OCDE (2017), Small, Medium, Strong. Trends in SME Performance and Business Conditions, Éditions OCDE, Paris, http://dx.doi. org/10.1787/9789264275683-en.

OCDE (2010), Structural and Demographic Business Statistics, Éditions OCDE, Paris, http://dx.doi.org/10.1787/9789264072886-en.

Système de comptabilité nationale (SCN) 2008, New York, http:// unstats.un.org/unsd/nationalaccount/sna2008.asp. 
Graphique 2.15. Valeur ajoutée par taille d'entreprise, secteur marchand En pourcentage de la valeur ajoutée totale, 2014 ou dernière année disponible

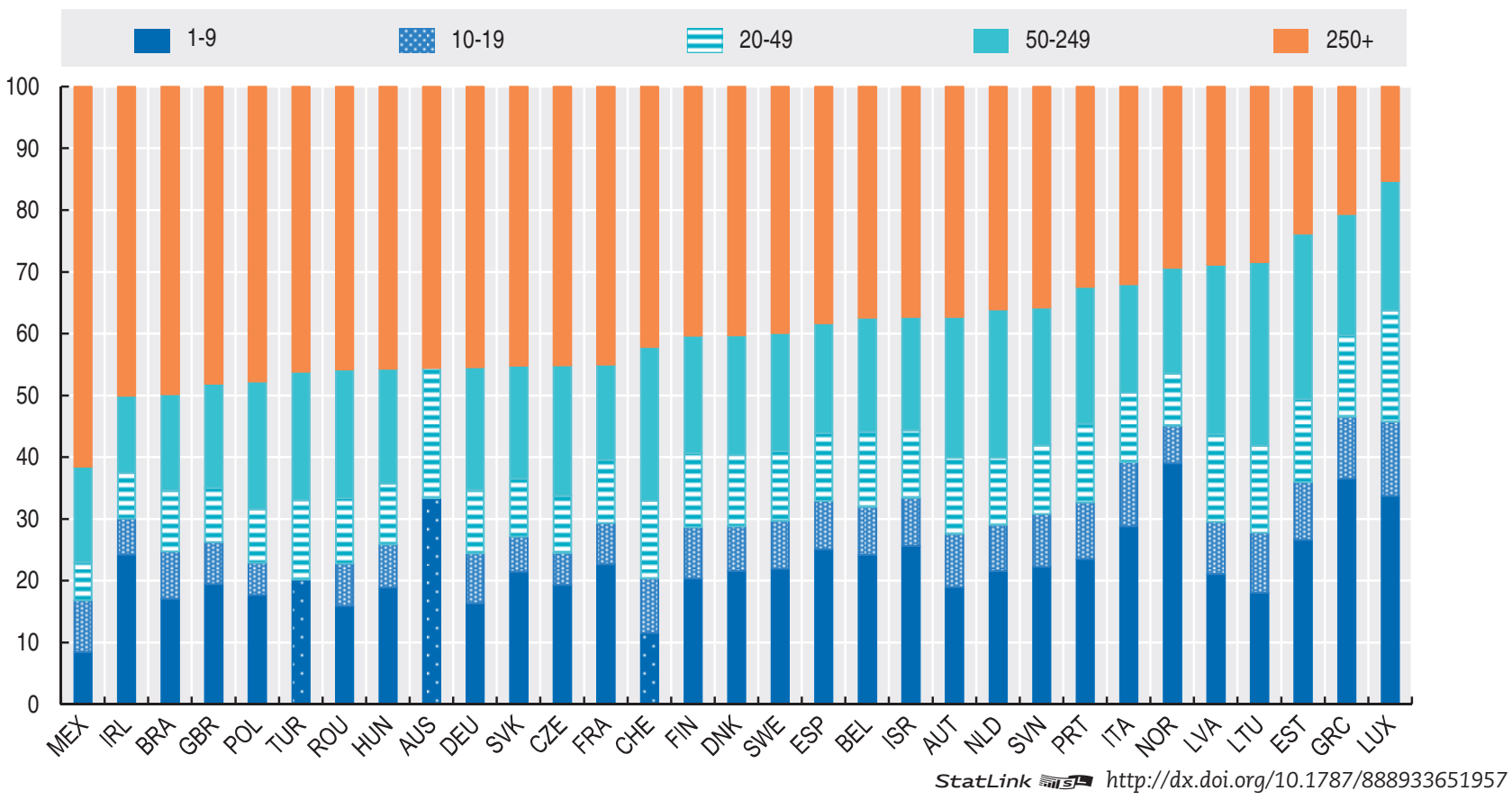

Graphique 2.16. Valeur ajoutée par taille d'entreprise, activités de fabrication

En pourcentage de la valeur ajoutée totale des activités de fabrication

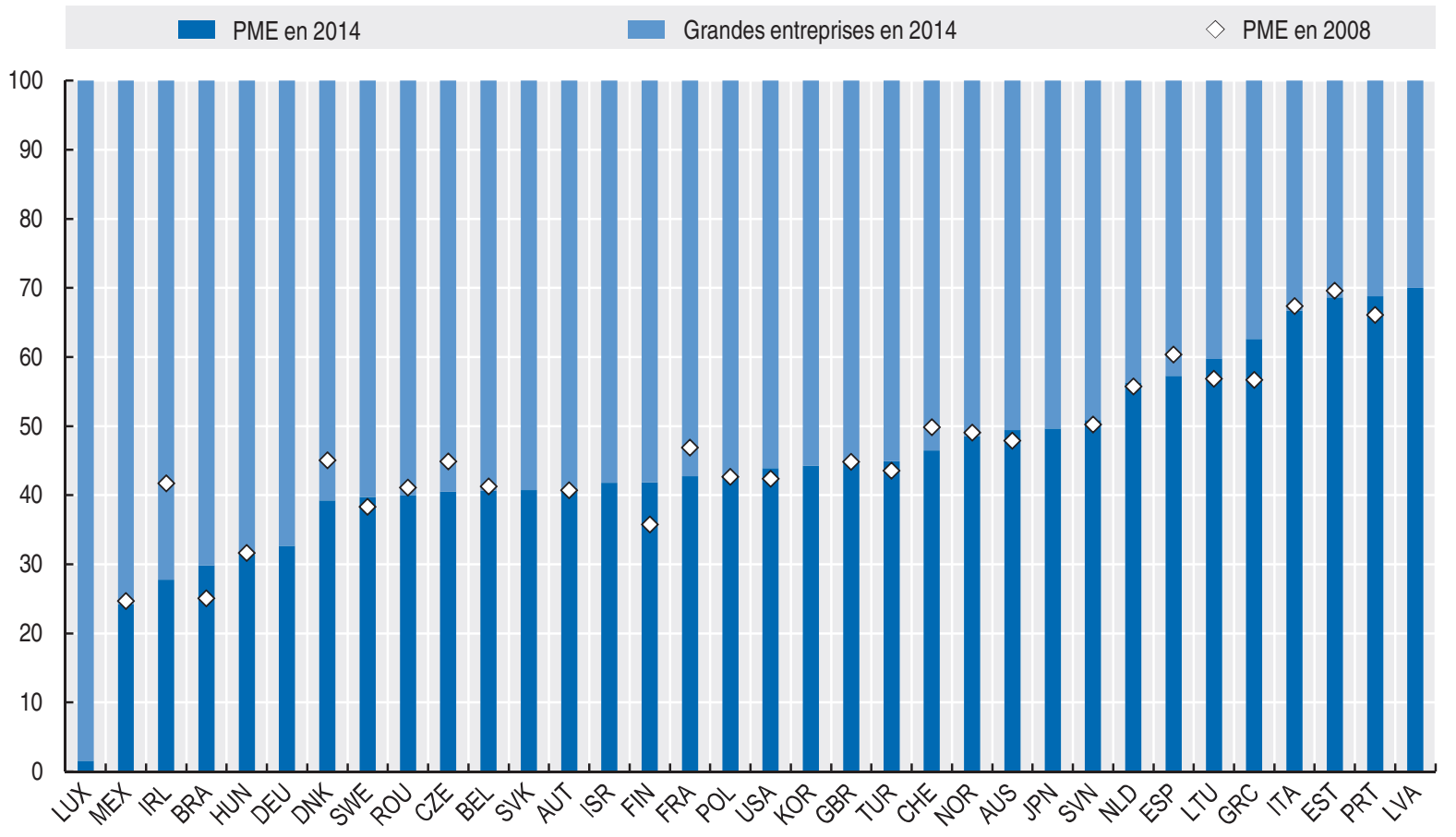

StatLink 部吉 $h$ ttp://dx.doi.org/10.1787/888933651976 
Graphique 2.17. Valeur ajoutée par taille d'entreprise et principaux secteurs En pourcentage de la valeur ajoutée totale, 2014 ou dernière année disponible

\section{Activités de fabrication}

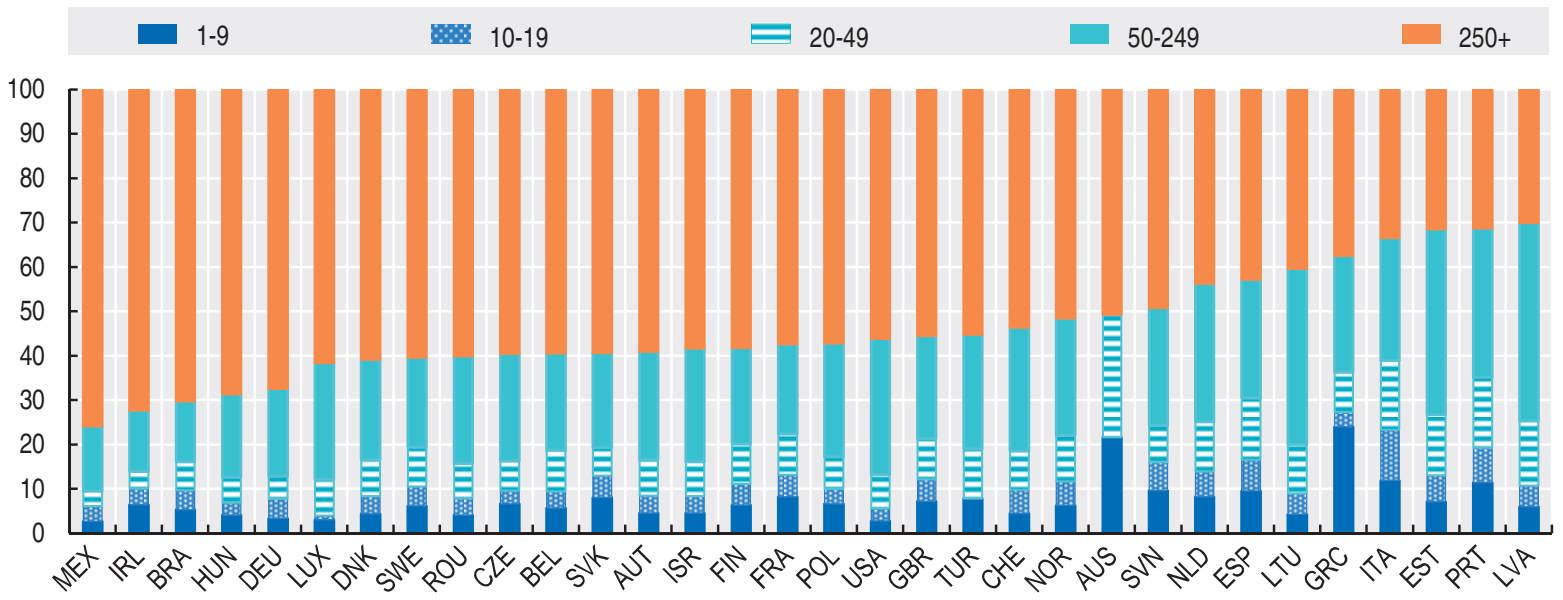

Services

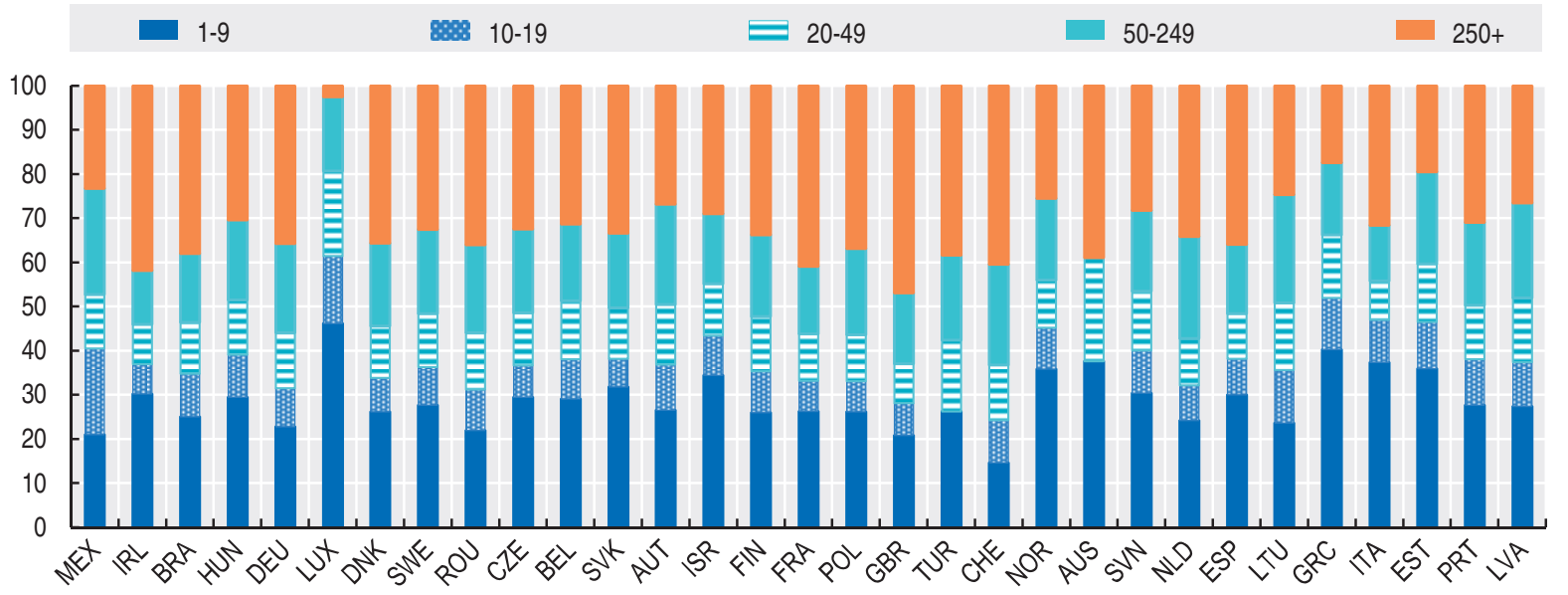

Construction

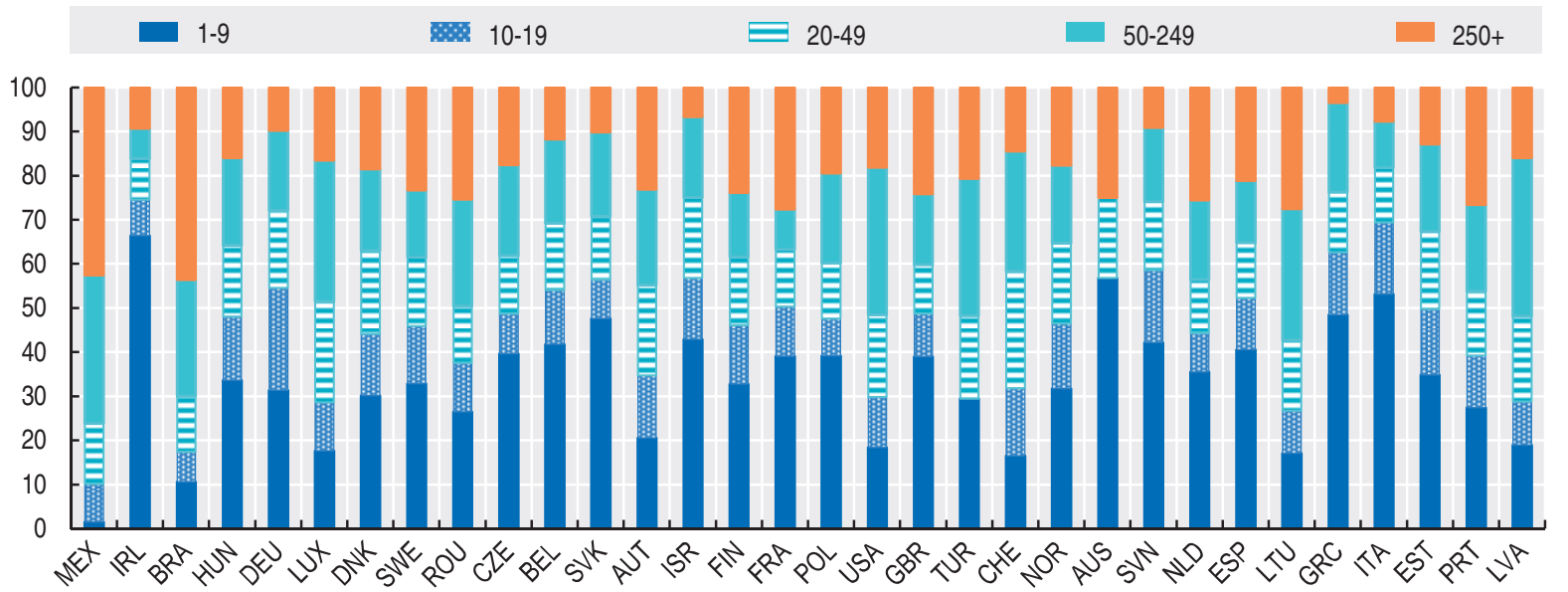

StatLink तiाls $h$ ttp://dx.doi.org/10.1787/888933651995 
Graphique 2.18. Valeur ajoutée des PME et des grandes entreprises par activité économique En pourcentage de la valeur ajoutée totale des PME (grandes entreprises), 2014 ou dernière année disponible

PME

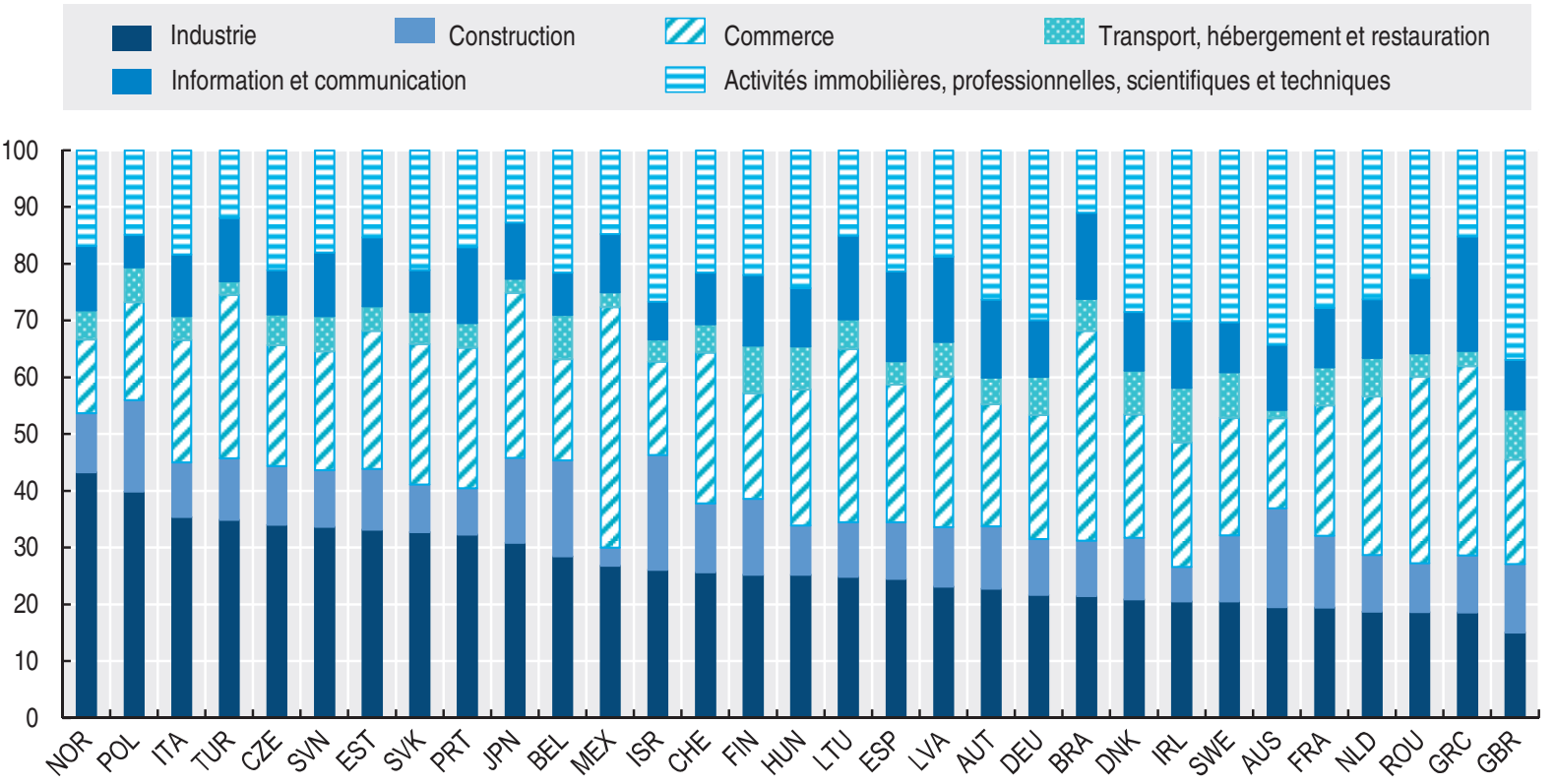

Grandes entreprises

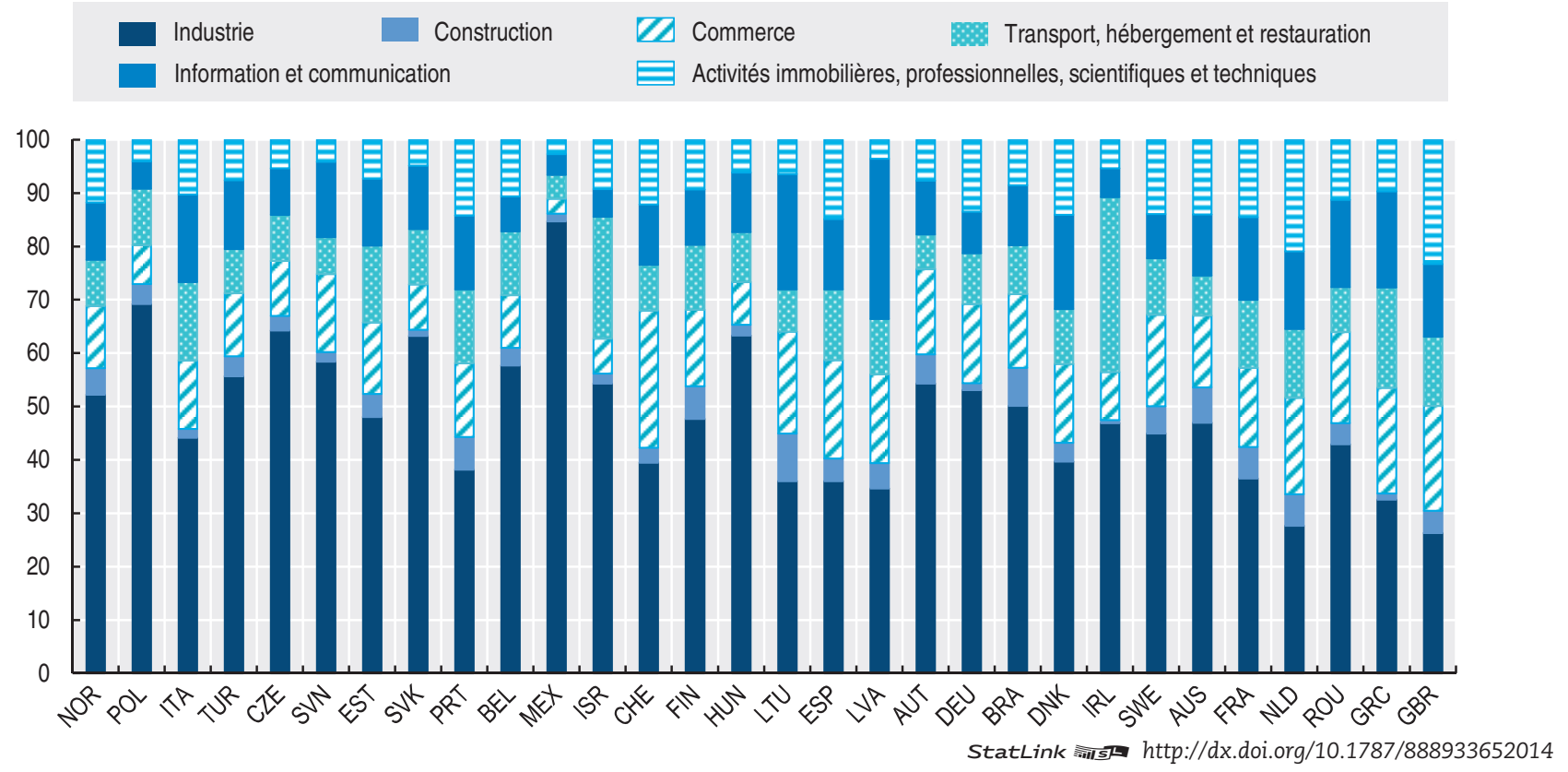




\section{Chiffre d'affaires par taille d'entreprise}

\section{À savoir}

- Dans les pays de l'OCDE, les PME représentent en moyenne $60 \%$ du chiffre d'affaires total, mais aux alentours de $80 \%$ dans des économies plus petites telles que le Luxembourg, l'Estonie et la Lettonie.

- En général, plus l'entreprise est grande, plus le chiffre d'affaires par personne occupée est élevé. Ce résultat reflète en partie une plus forte intensité capitalistique, mais les effets de composition et les structures actionnariales peuvent également jouer un rôle déterminant, ce qui signifie que, dans certaines économies, les entreprises plus petites réalisent un chiffre d'affaires par personne employée plus élevé. Par exemple, les entreprises de commerce de gros et de détail enregistrent généralement un chiffre d'affaires par personne employée plus élevé que d'autres entreprises, et les PME dépendantes (possédées par des groupes d'entreprises de plus grande taille) peuvent également afficher une intensité capitalistique relativement élevée (il s'agit souvent de propriété intellectuelle).

\section{Pertinence}

Le chiffre d'affaires des entreprises est une dimension utilisée seule ou en combinaison avec l'effectif pour définir les classes de taille aux fins d'élaboration des politiques. Ces classes de taille sont utilisées afin de déterminer, par exemple, le droit à une aide financière ou à d'autres programmes de soutien aux petites entreprises.

\section{Définitions}

Le chiffre d'affaires est défini comme le total des montants facturés par l'unité d'observation au cours de la période de référence, correspondant aux ventes marchandes de biens ou de services fournis à des tiers. Le chiffre d'affaires comprend tous les impôts et taxes grevant les biens ou services facturés par l'unité, à l'exception de la TVA facturée par l'unité à ses clients et des autres impôts déductibles assimilés et directement liés au chiffre d'affaires. Il comprend également tous les autres frais (transport, emballage, etc.) imputés aux clients, même si ceux-ci figurent séparément sur la facture et correspondent à un service assuré par l'unité. Les ristournes et rabais accordés aux clients, ainsi que la valeur des produits retournés, sont déduits des recettes perçues par l'unité dans le calcul du chiffre d'affaires. Le chiffre d'affaires exclut les recettes enregistrées dans les comptes dans les postes " autres produits d'exploitation ", " produits financiers " et " produits exceptionnels ". En sont également exclues les subventions d'exploitation reçues des pouvoirs publics ou d'autorités supranationales.

Le chiffre d'affaires dans chaque classe de taille est exprimé en pourcentage du chiffre d'affaires de l'ensemble des entreprises.

Le chiffre d'affaires par personne occupée s'obtient en divisant le chiffre d'affaires des entreprises de chaque classe de taille par le nombre de salariés correspondant.

\section{Comparabilité}

Compte tenu des pratiques différentes des pays en matière de collecte de données, la ventilation par classe de taille utilisée, à savoir 1-9, 10-19, 20-49, 50-249 et 250+, offre une comparabilité optimale. Certains pays utilisent des conventions différentes : pour le Mexique, "1-9 " correspond à " 1-10 ", " 10-19 " correspond à « 11-20 », « 20-49 » correspond à « 21-50 », « 50-249 » correspond à « 51-250 » et « 250+ » à « 251+ "; pour la Turquie, « 1-9 » correspond à « 1-19 »; pour l'Australie, « 1-9 » correspond à « 1-9 ", " 50-249 " à " 20-199 ", et « 250+ " à « 200+ ".

L'unité statistique utilisée pour le Mexique n'est pas l'entreprise, mais l'établissement. Pour les États-Unis, la Fédération de Russie et la Suisse, les données se réfèrent au nombre de salariés.

On observe une rupture de séries dans les données en 2013 pour la Finlande et le Portugal et en 2014 pour la France. Pour le Royaume-Uni, les données présentées n'incluent pas les quelque 2.6 millions de petites entreprises non enregistrées, c'est-à-dire des entreprises n'atteignant pas le seuil prévu pour le régime de la taxe sur la valeur ajoutée et/ou celui de la retenue de l'impôt à la source (pour les entreprises employant des salariés).

\section{Source}

OCDE, Statistiques structurelles et démographiques des entreprises (SDBS) (base de données), http://dx.doi.org/10.1787/sdbs-data-fr.

\section{Pour en savoir plus}

OCDE (2010), Structural and Demographic Business Statistics, Éditions OCDE, Paris, http://dx.doi.org/10.1787/9789264072886-en. 


\section{STRUCTURE ET PERFORMANCE DE LA POPULATION DES}

\section{Chiffre d'affaires par taille d'entreprise}

Graphique 2.19. Chiffre d'affaires par taille d'entreprise, secteur marchand Pourcentage du chiffre d'affaires total, 2014 ou dernière année disponible

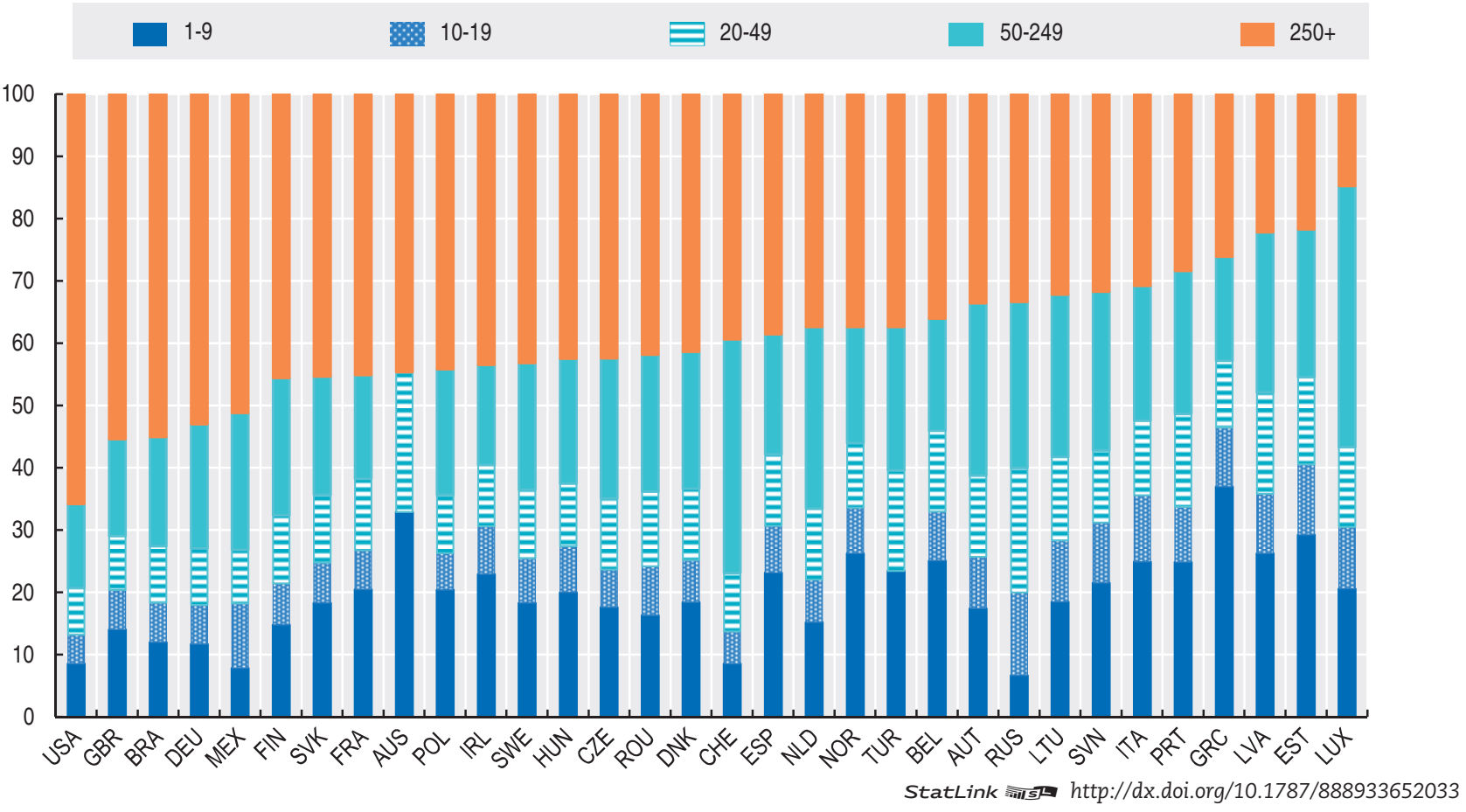

Graphique 2.20. Chiffre d'affaires par personne occupée, secteur marchand Milliers d'USD, PPA, 2014 ou dernière année disponible

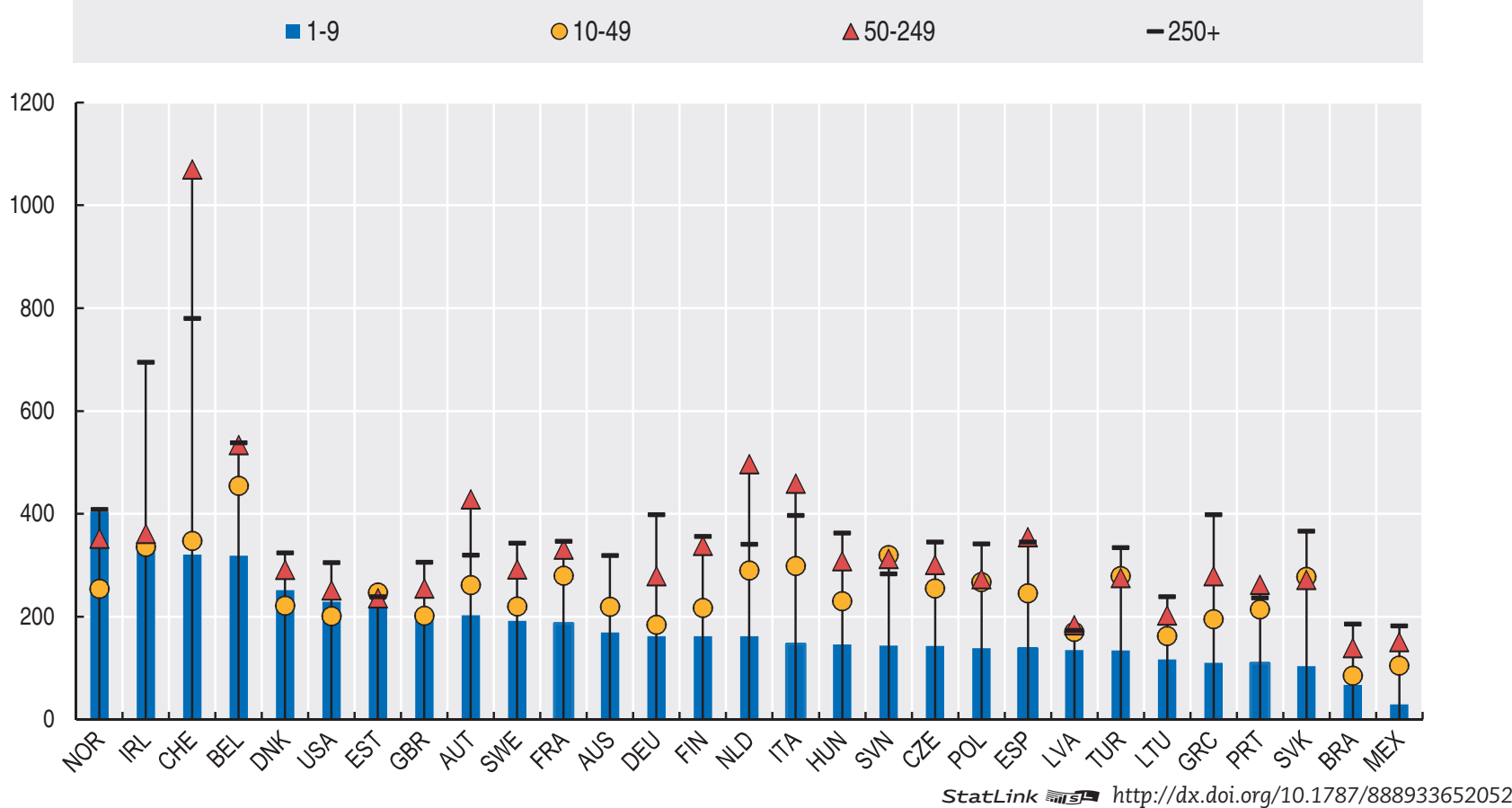




\section{Rémunération des salariés par taille d'entreprise}

\section{À savoir}

- Dans la plupart des pays, la rémunération des salariés constitue la plus grande part de la valeur ajoutée, en particulier dans les PME, qui ont généralement une intensité capitalistique moins forte que les grandes entreprises.

- La part de la rémunération des salariés dans la valeur ajoutée totale est particulièrement faible en Corée, en Irlande, au Japon et au Mexique, à la fois dans les entreprises de petite et de grande taille. Dans d'autres pays comme la Hongrie où les participations étrangères au capital des entreprises ou au contrôle des chaînes d'approvisionnement sont importantes, cette part est aussi généralement inférieure à la moyenne de l'OCDE. En revanche, en Allemagne et en France, la rémunération des salariés représente plus de $70 \%$ de la valeur ajoutée.

- Entre 2008 et 2014, la part de la rémunération des salariés dans la valeur ajoutée totale a diminué dans plusieurs pays, à la fois dans les PME et les grandes entreprises, notamment au Danemark, en République slovaque, en République tchèque, en Slovénie et dans les pays baltes, alors qu'elle a augmenté dans d'autres, comme la Finlande, la Grèce et l'Irlande.

\section{Pertinence}

Ces dernières années, une attention croissante a été accordée à la part du travail dans la valeur ajoutée, et plus particulièrement à l'impact des variations à la hausse ou à la baisse du différentiel capital-travail sur les inégalités.

\section{Définitions}

La rémunération des salariés comprend la rémunération totale, en espèces ou en nature, versée aux salariés en paiement du travail accompli par ces derniers au cours de la période de référence. Aucune rémunération n'est payable pour le travail non rémunéré accompli sur une base volontaire, notamment le travail accompli par les membres d'un ménage au sein d'une entreprise non constituée en société et détenue par ce même ménage. La rémunération des salariés n'inclut pas les taxes payables par l'employeur sur les traitements et salaires. Elle comprend donc les traitements et salaires des salariés et les autres cotisations patronales.

La rémunération du travail pour toutes les personnes occupées est équivalente à la somme des traitements et salaires de toutes les personnes salariées et des autres cotisations patronales versées au bénéfice des salariés.

Informations sur les données concernant Israël : http:// dx.doi.org/10.1787/888932315602.

\section{Comparabilité}

De nombreuses PME sont des entreprises non constituées en société. Leurs propriétaires ne se versent pas de salaire, mais reçoivent une rémunération sous forme de revenu mixte (selon la définition du Système de comptabilité nationale de 2008), qui est un élément de la valeur ajoutée. Les estimations qui ne se basent que sur la rémunération des salariés rapportée à la valeur ajoutée totale ont donc toutes les chances, par rapport aux estimations concernant les grandes entreprises, de sousestimer la contribution relative du travail dans les PME. Ainsi peuvent s'expliquer les parts moindres observées par exemple pour l'Italie et la Lettonie.

Les données relatives à l'Australie, au Brésil et à Israël font référence à la rémunération de l'ensemble des personnes occupées. Les données concernant les États-Unis se rapportent aux établissements.

On observe une rupture de séries dans les données en 2013 pour la Finlande et le Portugal et en 2014 pour la France. Pour le Royaume-Uni, les données présentées n'incluent pas les quelque 2.6 millions de petites entreprises non enregistrées, c'est-à-dire des entreprises n'atteignant pas le seuil prévu pour le régime de la taxe sur la valeur ajoutée et/ou celui de la retenue de l'impôt à la source (pour les entreprises employant des salariés).

\section{Source}

OCDE, Statistiques structurelles et démographiques des entreprises (SDBS) (base de données), http://dx.doi.org/10.1787/sdbs-data-fr.

\section{Pour en savoir plus}

OCDE (2017), OECD Compendium of Productivity Indicators 2017, Éditions OCDE, Paris, http://dx.doi.org/10.1787/pdtuy-2017-en.

OCDE (2017), Small, Medium, Strong. Trends in SME Performance and Business Conditions, Éditions OCDE, Paris, http://dx.doi.org/ 10.1787/9789264275683-en.

OCDE (2015), Perspectives de l'emploi de l'OCDE 2015, Éditions OCDE, Paris, http://dx.doi.org/10.1787/empl_outlook-2015-fr.

OCDE (2010), Structural and Demographic Business Statistics, Éditions OCDE, Paris, http://dx.doi.org/10.1787/9789264072886-en. 
Graphique 2.21. Rémunération des salariés rapportée à la valeur ajoutée, par taille d'entreprise, activités de fabrication

En pourcentage

$\square 2014$

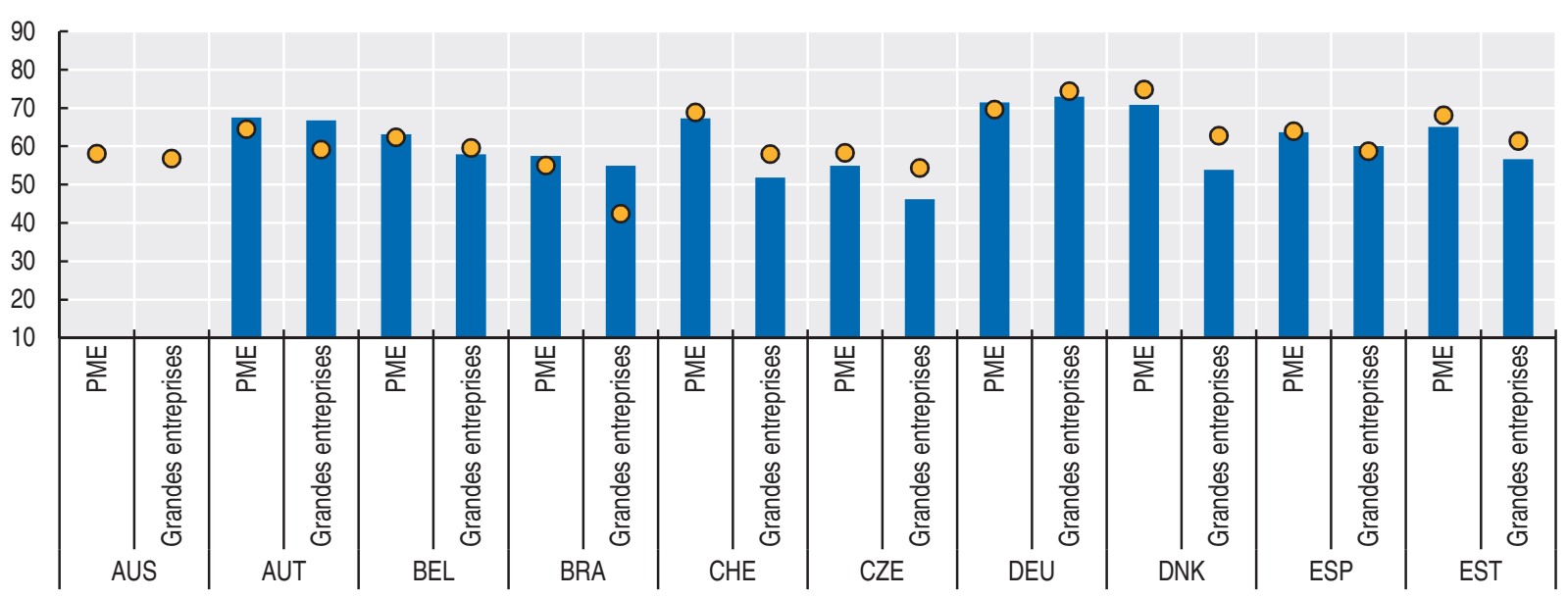

- 2014

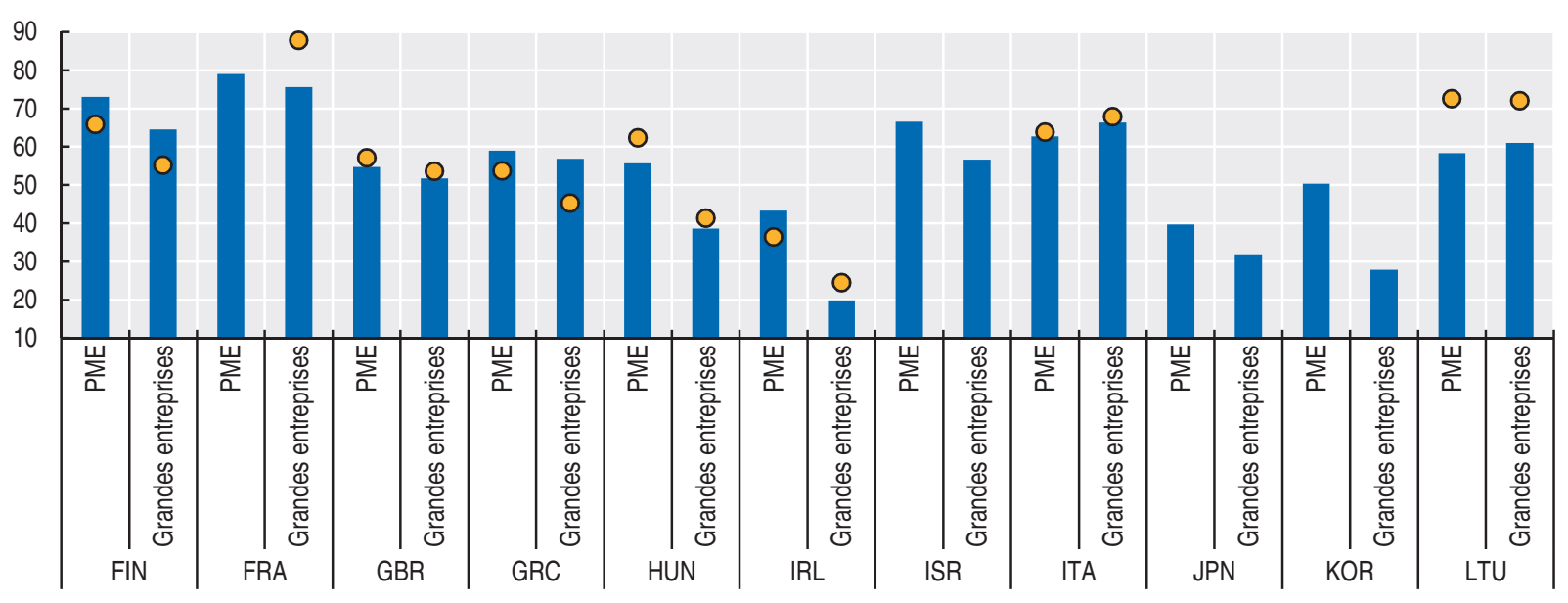

- 2014

02008

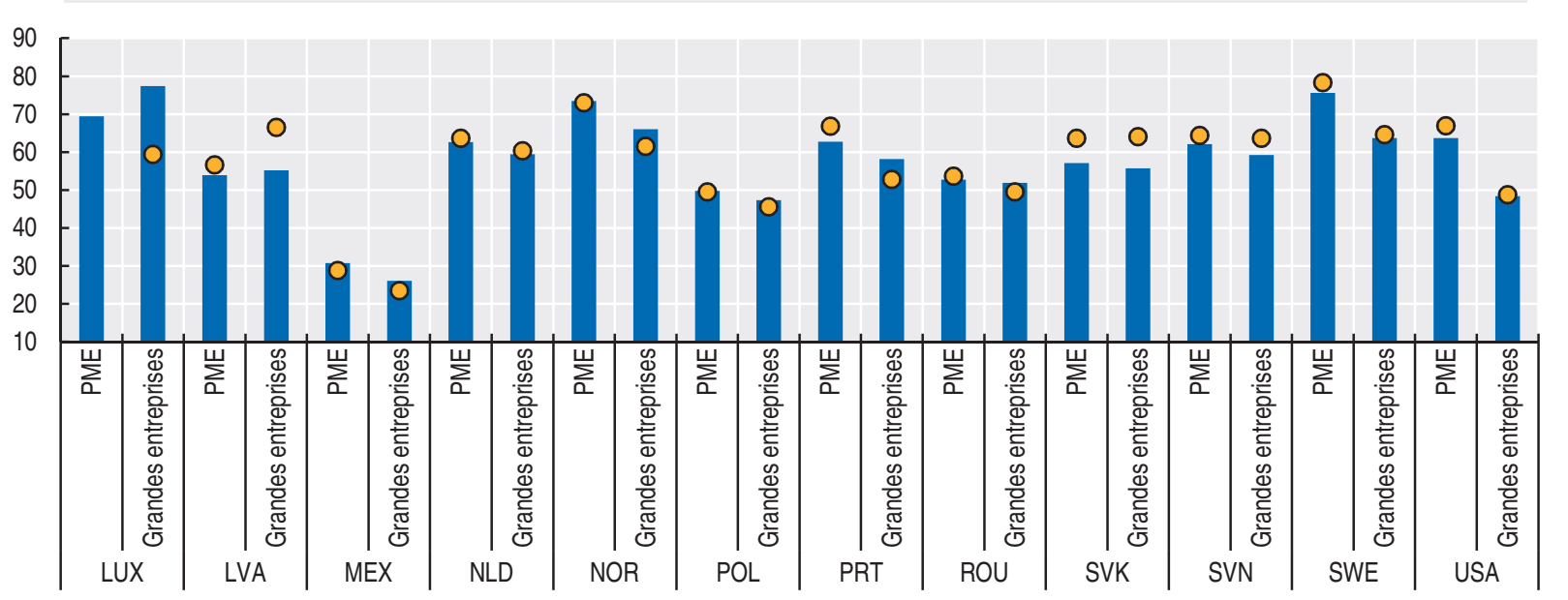

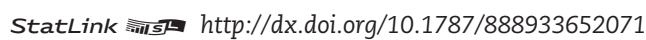



3. PRODUCTIVITÉ PAR TAILLE D'ENTREPRISE

Écarts de productivité entre les entreprises

Croissance de la productivité par taille d'entreprise

Productivité et écarts de salaire entre les entreprises

PANORAMA DE L'ENTREPRENEURIAT 2017 ๑ OCDE 2018

61 


\section{À savoir}

- Dans le secteur manufacturier, où la production a tendance à avoir une plus forte intensité capitalistique et où les plus grandes entreprises savent tirer parti des rendements d'échelle croissants, les entreprises de grande taille affichent presque toujours des niveaux de productivité plus élevés que les entreprises plus petites.

- La taille relative (ou dispersion) des écarts de productivité entre grandes et petites entreprises varie considérablement suivant les pays. Au Royaume-Uni, par exemple, la productivité des microentreprises du secteur manufacturier équivaut à environ $60 \%$ de celle des grandes entreprises, contre environ 20 \% en Turquie et en Hongrie. De même, les PME du secteur des services affichent une productivité similaire à celle des grandes entreprises en Finlande et en Suède, tandis qu'au Mexique, elle y est inférieure dans une proportion comprise entre $25 \%$ et $75 \%$.

- Les écarts de productivité entre les classes de taille sont relativement moindres dans le secteur des services. Dans beaucoup de pays, les entreprises de taille moyenne enregistrent de meilleurs résultats que les grandes entreprises, ce qui s'explique par l'avantage concurrentiel qu'elles détiennent dans des activités de niche, à forte notoriété ou à haute teneur en contenu protégé par la propriété intellectuelle, ainsi que par leur utilisation intensive de TIC abordables.

\section{Pertinence}

La productivité mesure l'efficience de l'allocation des ressources dans une économie. Le problème est que les analyses ne s'intéressent généralement qu'à ce qui se passe au niveau sectoriel (activités), sans tenir compte des différences de productivité entre les entreprises d'un même secteur, en particulier de la contribution des PME, considérées comme des moteurs importants de la croissance à mesure qu'elles s'agrandissent. Des statistiques plus détaillées montrant les contributions relatives par classe de taille peuvent donner une meilleure idée de cette hétérogénéité et permettre l'élaboration de politiques publiques mieux ciblées, afin de réduire les obstacles à la croissance de la productivité et de tirer parti des possibilités qui existent pour la développer.

\section{Définitions}

La productivité du travail est la valeur ajoutée brute aux prix courants par personne occupée. Pour la définition des " activités de fabrication " et des "services ", se reporter au Guide de lecture. Les services financiers étant exclus, il importe d'être prudent au moment d'extrapoler les résultats et de tirer des conclusions sur l'ensemble des activités du secteur marchand dans les différents pays, en particulier ceux dans lesquels les activités de services financiers sont relativement importantes comme le Luxembourg, le Royaume-Uni et la Suisse.

La productivité du travail par taille d'entreprise exprimée en monnaie nationale est convertie en USD à partir des parités de pouvoir d'achat (PPA) pour le PIB.

Informations sur les données concernant Israël : http:// dx.doi.org/10.1787/888932315602.

\section{Comparabilité}

Les données sur la valeur ajoutée correspondent à la valeur ajoutée au coût des facteurs dans les pays de l'UE et aux prix de base pour les autres pays. Les estimations de la valeur ajoutée et de l'emploi présentées par taille d'entreprise sont établies à partir de la base de données de l'OCDE sur les statistiques structurelles et démographiques des entreprises, et ne coïncident généralement pas avec les estimations produites selon le Système de comptabilité nationale. Ce dernier prévoit en effet un certain nombre d'ajustements destinés à prendre en considération des entreprises et activités potentiellement absentes des statistiques structurelles sur les entreprises, comme les très petites unités ou les travailleurs indépendants, ou à tenir compte de l'économie non observée.

La comparabilité des classes de taille, secteurs et pays peut pâtir des disparités de la part de l'emploi à temps partiel dans l'emploi total. C'est pourquoi, dans l'analyse de la productivité, la mesure privilégiée du facteur travail est le nombre total d'heures travaillées et non l'effectif, mais on ne dispose généralement pas de ventilation de ces données par taille d'entreprise. Des lacunes dans les données imputables aux règles de confidentialité en vigueur dans les pays concernés peuvent également nuire à la comparabilité internationale.

Compte tenu des pratiques différentes des pays en matière de collecte de données, la ventilation par classe de taille utilisée, à savoir 1-9, 10-19, 20-49, 50-249 et 250+, offre une comparabilité optimale. Certains pays utilisent des conventions différentes : pour l'Australie, " 1-9 " correspond à " 1-19 ", " 20-49 » correspond à « 20-199 », et « 250+ » correspond à « 200+ »; pour le Mexique, «1-9 » correspond à «1-10 ", « 10-19 " correspond à « 11-20 », « 20-49 » correspond à « 21-50 », « 50-249 » correspond à « 51-250 » et « 250+ » correspond à « 251+ "; pour la Turquie, « 1-9 » correspond à «1-19».

Les données concernant la Suisse et les États-Unis se rapportent au nombre de salariés. L'unité statistique utilisée pour le Mexique n'est pas l'entreprise, mais l'établissement. Pour le Royaume-Uni, les données présentées n'incluent pas les quelque 2.6 millions de petites entreprises non enregistrées, c'est-à-dire des entreprises n'atteignant pas le seuil prévu pour le régime de la taxe sur la valeur ajoutée et/ou celui de la retenue de l'impôt à la source (pour les entreprises employant des salariés).

\section{Sources}

OCDE, Statistiques structurelles et démographiques des entreprises (SDBS) (base de données), http://dx.doi.org/10.1787/sdbs-data-fr.

Statistiques de l'OCDE sur les comptes nationaux (base de données), http://dx.doi.org/10.1787/na-data-fr.

Statistiques de l'OCDE sur la productivité (base de données), http://dx.doi.org/10.1787/pdtuy-data-fr.

\section{Pour en savoir plus}

OCDE (2017), OECD Compendium of Productivity Indicators 2017, Éditions OCDE, Paris, http://dx.doi.org/10.1787/pdtuy-2017-en

OCDE (2017), Small, Medium, Strong. Trends in SME Performance and Business Conditions, Éditions OCDE, Paris, http://dx.doi. org/10.1787/9789264275683-en

OCDE (2001), Mesurer la productivité - Manuel de l'OCDE Mesurer la croissance de la productivité par secteur et pour l'ensemble de l'économie, Éditions OCDE, Paris, http://dx.doi. org/10.1787/9789264294516-fr. 
Graphique 3.1. Productivité du travail par taille d'entreprise, secteur marchand

Valeur ajoutée par personne occupée, milliers d'USD, PPA, 2014, ou dernière année disponible

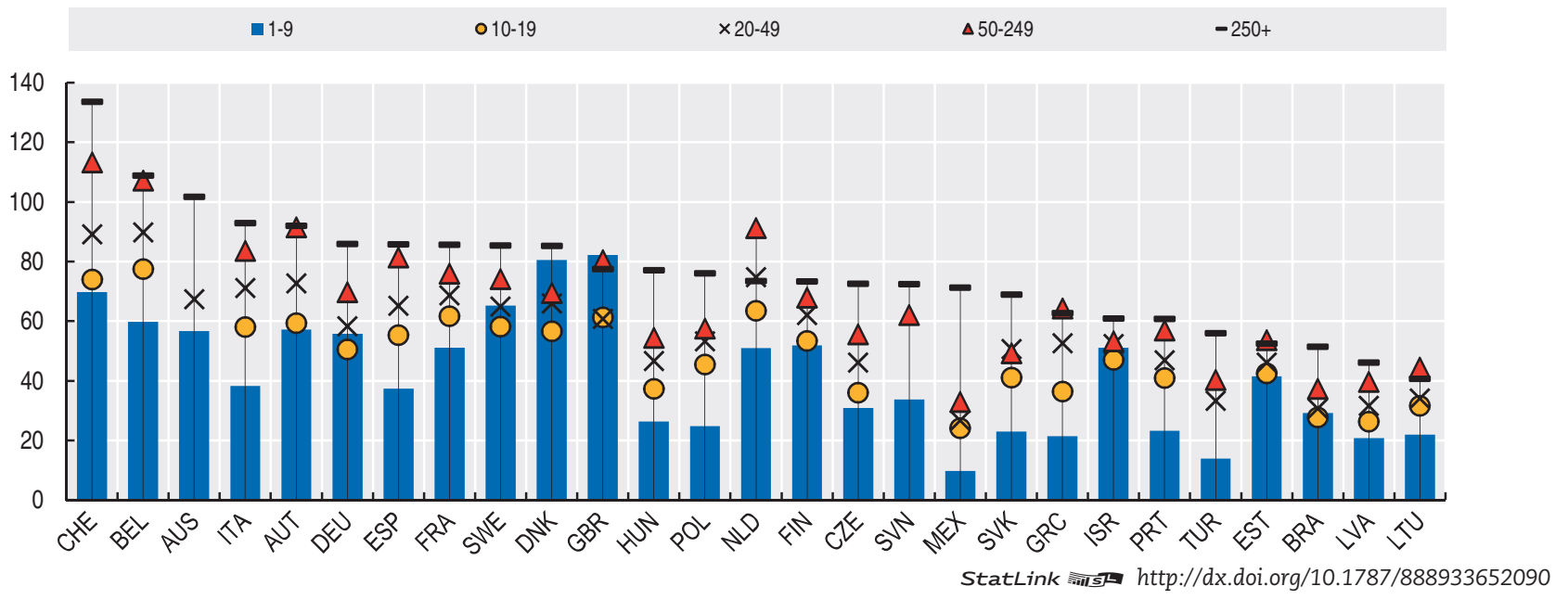

Graphique 3.2. Productivité du travail par taille d'entreprise, activités de fabrication et services Valeur ajoutée par personne occupée, indice $250+=100,2014$, ou dernière année disponible

Activités de fabrication

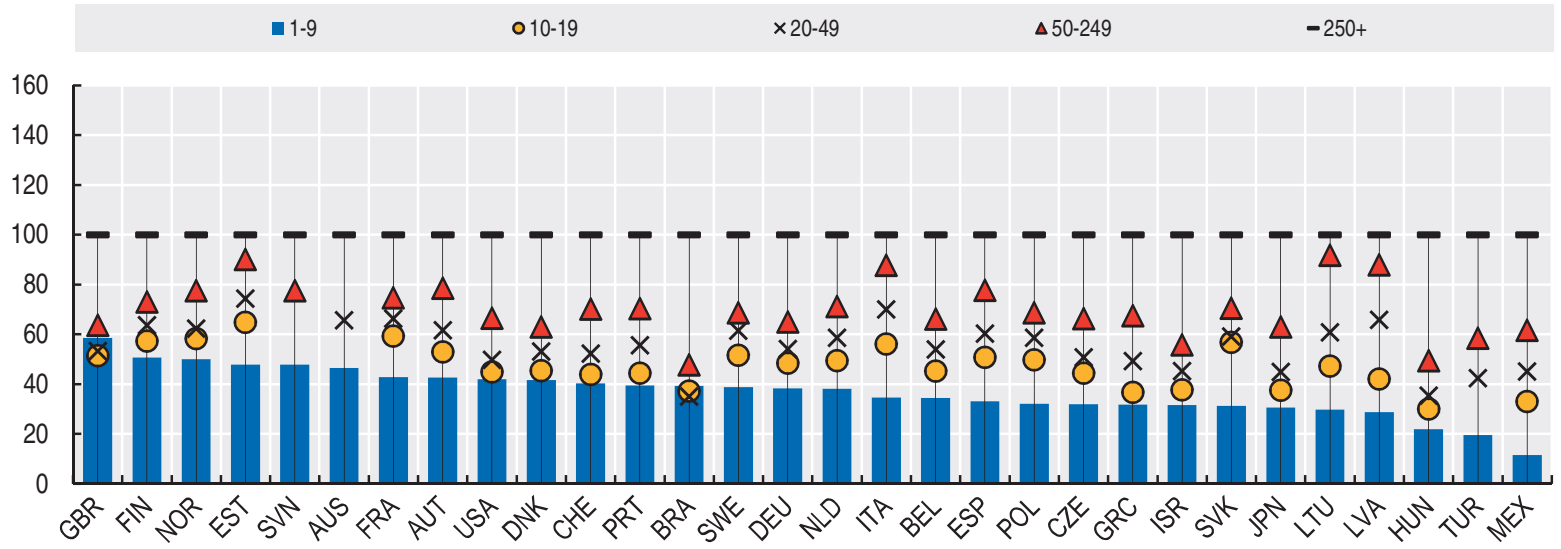

Services

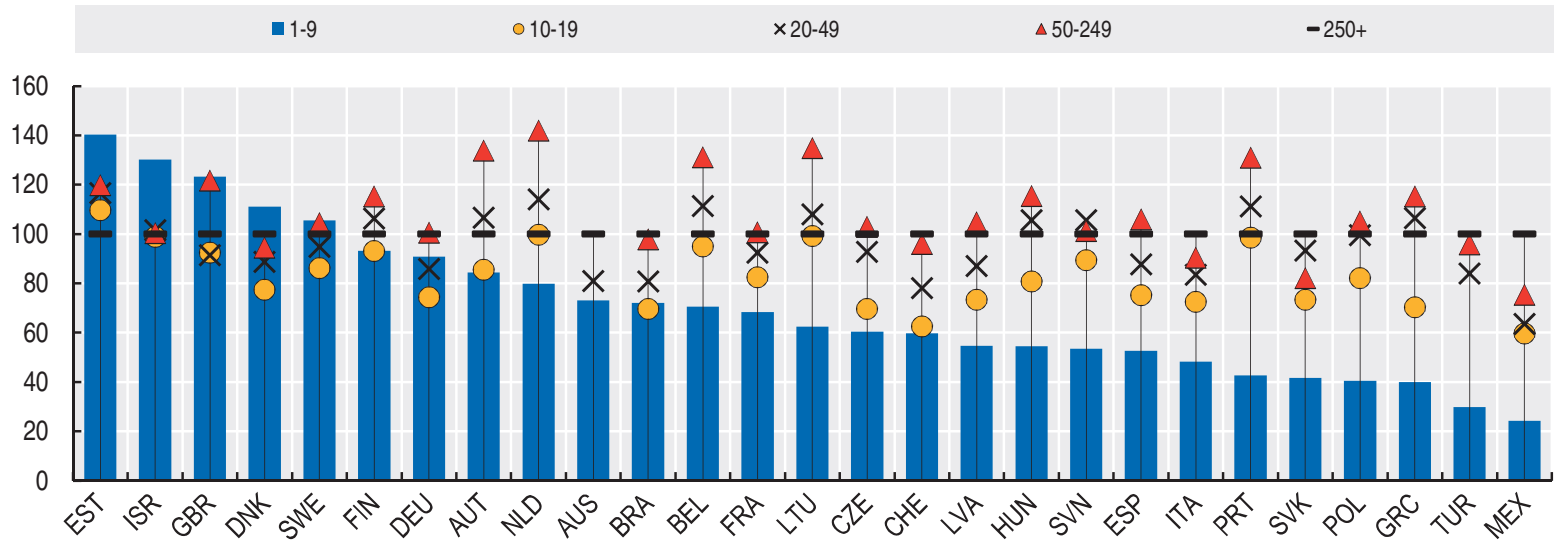

StatLink ails http://dx.doi.org/10.1787/888933652109 


\section{À savoir}

- Après la crise, dans un grand nombre de pays, les PME du secteur manufacturier ont vu leur productivité croître plus lentement que les grandes entreprises, ce qui a creusé les écarts préexistants, en particulier en Allemagne, en Belgique, en Lettonie, en République slovaque et en République tchèque. En Lituanie, en Pologne et en Turquie, bien que le retard reste important, la productivité des PME a crû beaucoup plus fortement que celle des grandes entreprises.

- En Espagne et au Portugal, la croissance de la productivité du travail enregistrée par les PME du secteur manufacturier va de pair avec un recul de l'emploi et de la valeur ajoutée, ce qui donne à penser que les disparitions d'entreprises ou d'activités peu performantes sont probablement pour beaucoup dans la hausse globale de la productivité du travail.

- Dans le secteur des services, la situation est plus contrastée : après la crise, dans nombre de pays, les PME ont vu leur productivité croître plus vite, mais leurs effectifs plus lentement que les grandes entreprises.

\section{Pertinence}

La performance d'une entreprise dépend de toute une série de facteurs, dont sa taille et son secteur d'activité. Si les grandes entreprises ont tendance à être plus productives que les petites, la croissance de la productivité dans les petites structures peut s'expliquer par l'utilisation intensive de technologies de l'information et des communications (TIC) abordables, ainsi que par l'avantage concurrentiel qu'elles détiennent dans des activités de niche, à forte notoriété ou à haute teneur en contenu protégé par la propriété intellectuelle.

\section{Définitions}

Pour mesurer la productivité du travail, on divise la valeur ajoutée brute aux prix courants par personne occupée, tirée de la base de données de l'OCDE sur les statistiques structurelles et démographiques des entreprises, par l'indice d'ajustement sectoriel provenant de la base de données Statistiques de l'OCDE sur les comptes nationaux.

Pour une définition des " activités de fabrication » et des " services ", se reporter au Guide de lecture.

\section{Comparabilité}

Les données sur la valeur ajoutée correspondent à la valeur ajoutée au coût des facteurs dans les pays de l'UE et aux prix de base pour les autres pays. Les estimations de la valeur ajoutée et de l'emploi présentées par taille d'entreprise sont établies à partir de la base de données de l'OCDE sur les statistiques structurelles et démographiques des entreprises et ne coïncident généralement pas avec les estimations produites selon le Système de comptabilité nationale. Ce dernier prévoit en effet un certain nombre d'ajustements destinés à prendre en considération des entreprises et activités potentiellement absentes des statistiques structurelles sur les entreprises, comme les microentreprises ou les travailleurs indépendants, ou à tenir compte de l'économie non observée.

La comparabilité des classes de taille, secteurs et pays peut pâtir des disparités de la part de l'emploi à temps partiel dans l'emploi total. C'est pourquoi, dans l'analyse de la productivité, la mesure privilégiée du facteur travail est le nombre total d'heures travaillées et non l'effectif, mais on ne dispose généralement pas de ventilation de ces données par taille d'entreprise. Des lacunes dans les données imputables aux règles de confidentialité en vigueur dans les pays concernés peuvent également nuire à la comparabilité internationale.

Parce qu'elles ne s'appuient pas sur des cohortes fixes d'entreprises, les estimations présentées ici donnent une image faussement élevée de la croissance de la productivité dans les grandes entreprises, et une image faussement faible de celle des PME : les PME qui affichent au démarrage une forte croissance de leur productivité sont en effet plus susceptibles de devenir de grandes entreprises, tandis que les grandes entreprises enregistrant une faible productivité ont plus de chances de se contracter et de devenir des PME.

Pour le Royaume-Uni, les données présentées n'incluent pas les quelque 2.6 millions de petites entreprises non enregistrées, c'est-à-dire des entreprises n'atteignant pas le seuil prévu pour le régime de la taxe sur la valeur ajoutée et/ou celui de la retenue de l'impôt à la source (pour les entreprises employant des salariés). Les graphiques 3.3. à 3.6. couvrent la période 2009-14, et non 2008-14 comme dans d'autres parties de cet ouvrage, en raison de problèmes de disponibilité des données. Les taux de croissance de l'emploi indiqués sur les graphiques 3.5 et 3.6 peuvent différer de ceux communiqués dans le chapitre 2 car ils ne couvrent que les activités pour lesquelles des statistiques sur la valeur ajoutée sont également disponibles.

\section{Sources}

OCDE, Statistiques structurelles et démographiques des entreprises (SDBS) (base de données), http://dx.doi.org/10.1787/sdbs-data-fr.

Statistiques de l'OCDE sur les comptes nationaux (base de données), http://dx.doi.org/10.1787/na-data-fr.

Statistiques de l'OCDE sur la productivité (base de données), http:// dx.doi.org/10.1787/pdtuy-data-fr.

\section{Pour en savoir plus}

OCDE (2017), OECD Compendium of Productivity Indicators 2017, Éditions OCDE, Paris, http://dx.doi.org/10.1787/pdtuy-2017-en

Hsieh, C. (2015), « Policies for Productivity Growth », OECD Productivity Working Papers, $n^{\circ}$ 3, Éditions OCDE, Paris, http:// dx.doi.org/10.1787/5jrp1f5rddtc-en.

OCDE (2001), Mesurer la productivité - Manuel de l'OCDE : Mesurer la croissance de la productivité par secteur et pour l'ensemble de l'économie, Éditions OCDE, Paris, http://dx.doi. org/10.1787/9789264294516-fr. 


\section{PRODUCTIVITÉ PAR TAILLE D’ENTREPRISE}

Groissance de la productivité par taille d’entreprise

Graphique 3.3. Croissance de la productivité du travail par taille d'entreprise, activités de fabrication Valeur ajoutée réelle par personne occupée, taux annuel moyen, 2009-14

- PME (1-249)

- Grandes entreprises (250+)

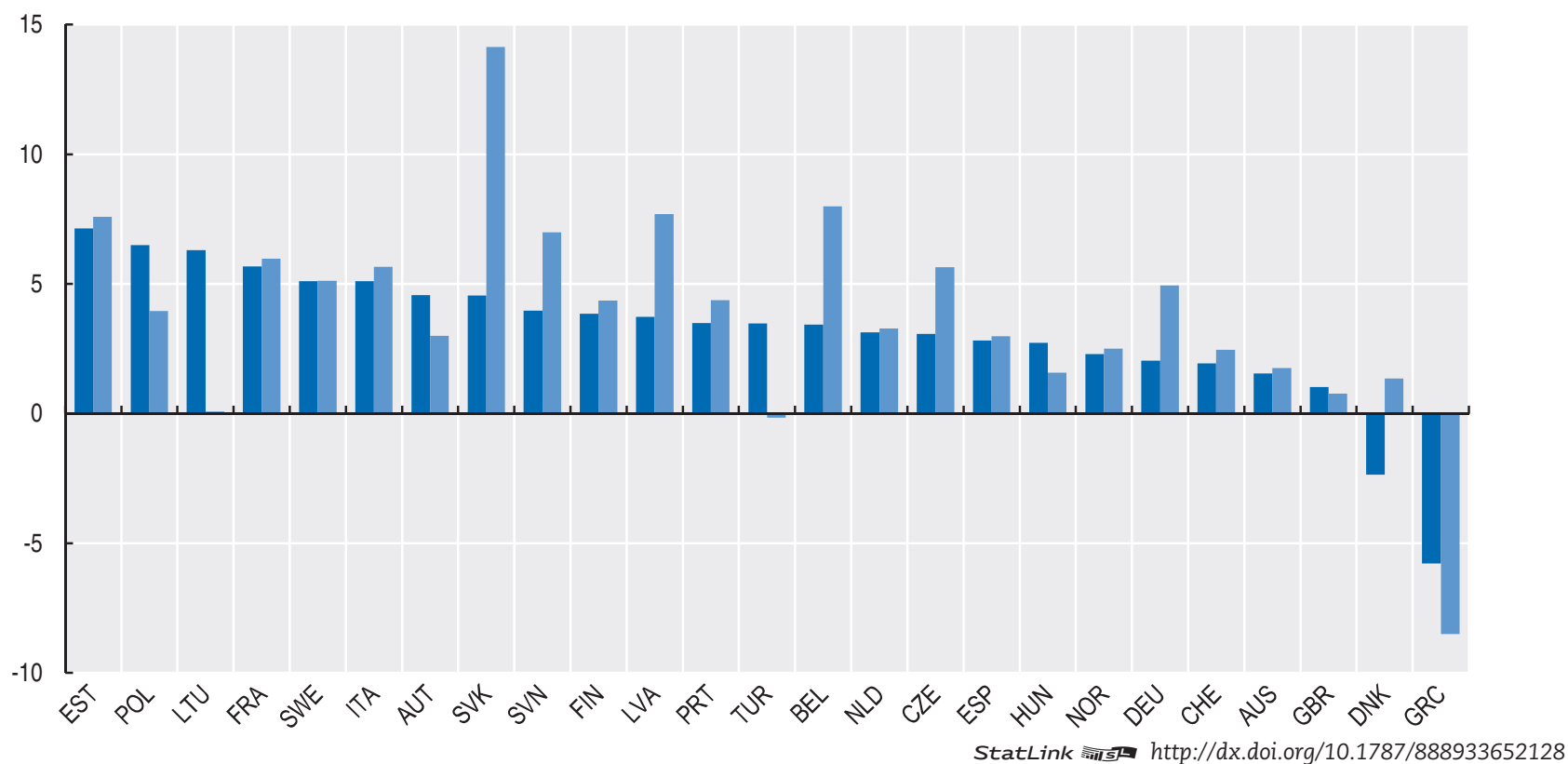

Graphique 3.4. Croissance de la productivité du travail par taille d'entreprise, services Valeur ajoutée réelle par personne occupée, taux annuel moyen, 2009-14

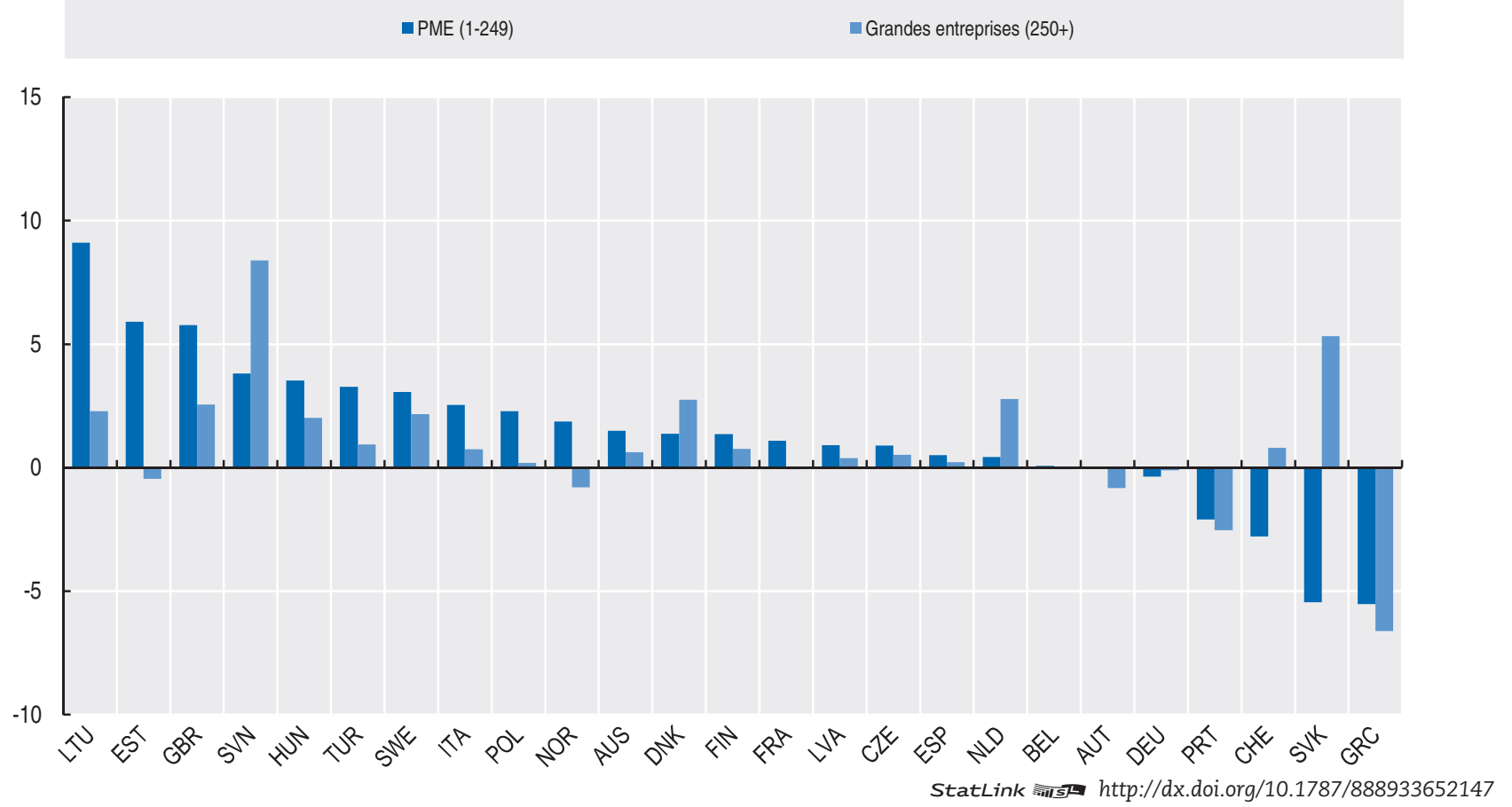




\section{PRODUCTIVITÉ PAR TAILLE D’ENTREPRISE}

\section{Groissance de la productivité par taille d'entreprise}

Graphique 3.5. Croissance de la valeur ajoutée réelle et de l'emploi par taille d'entreprise, activités de fabrication Taux annuel moyen, pourcentage, 2009-14

Petites et moyennes entreprises (1-249 salariés)

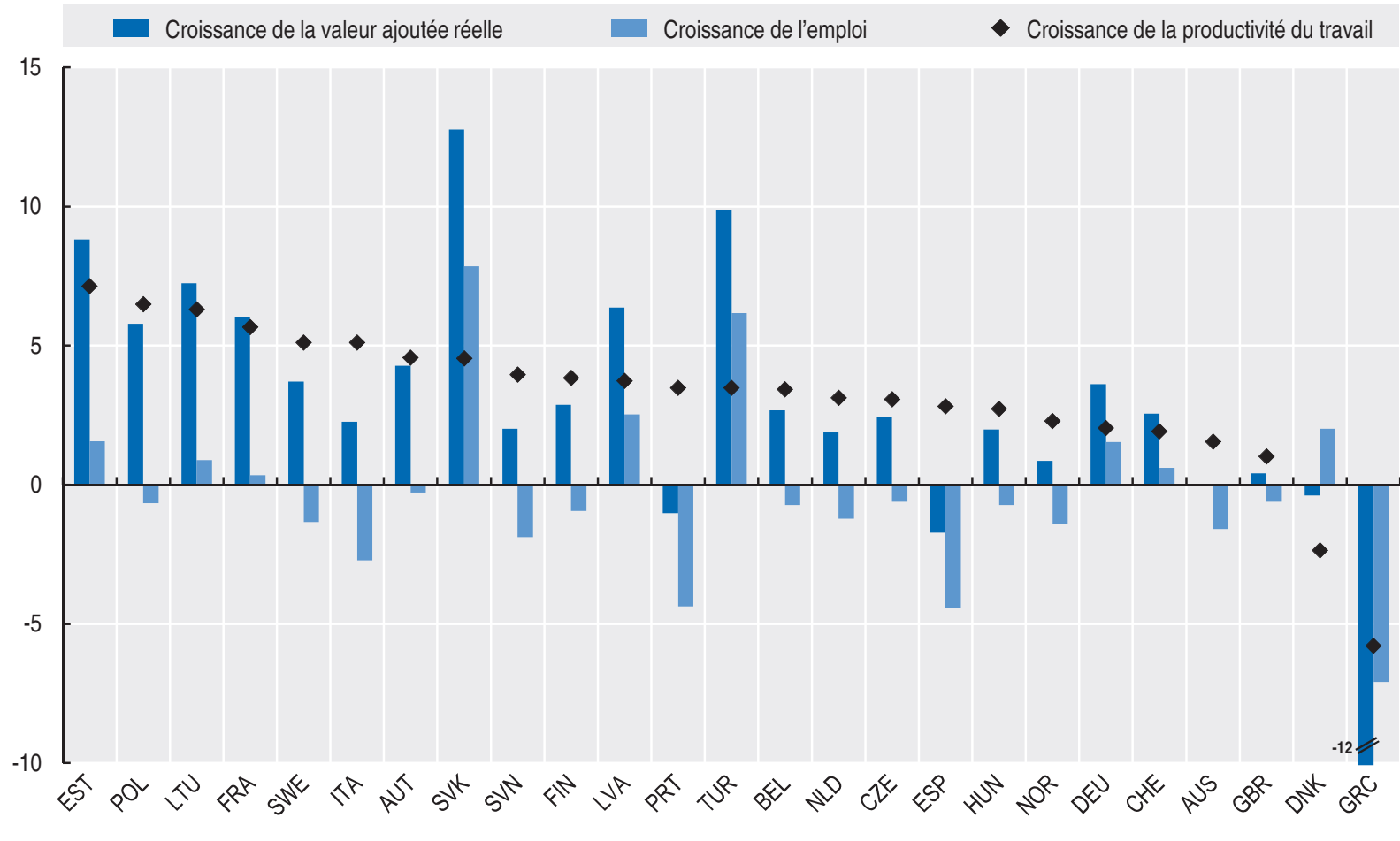

Grandes entreprises (250 salariés ou plus)

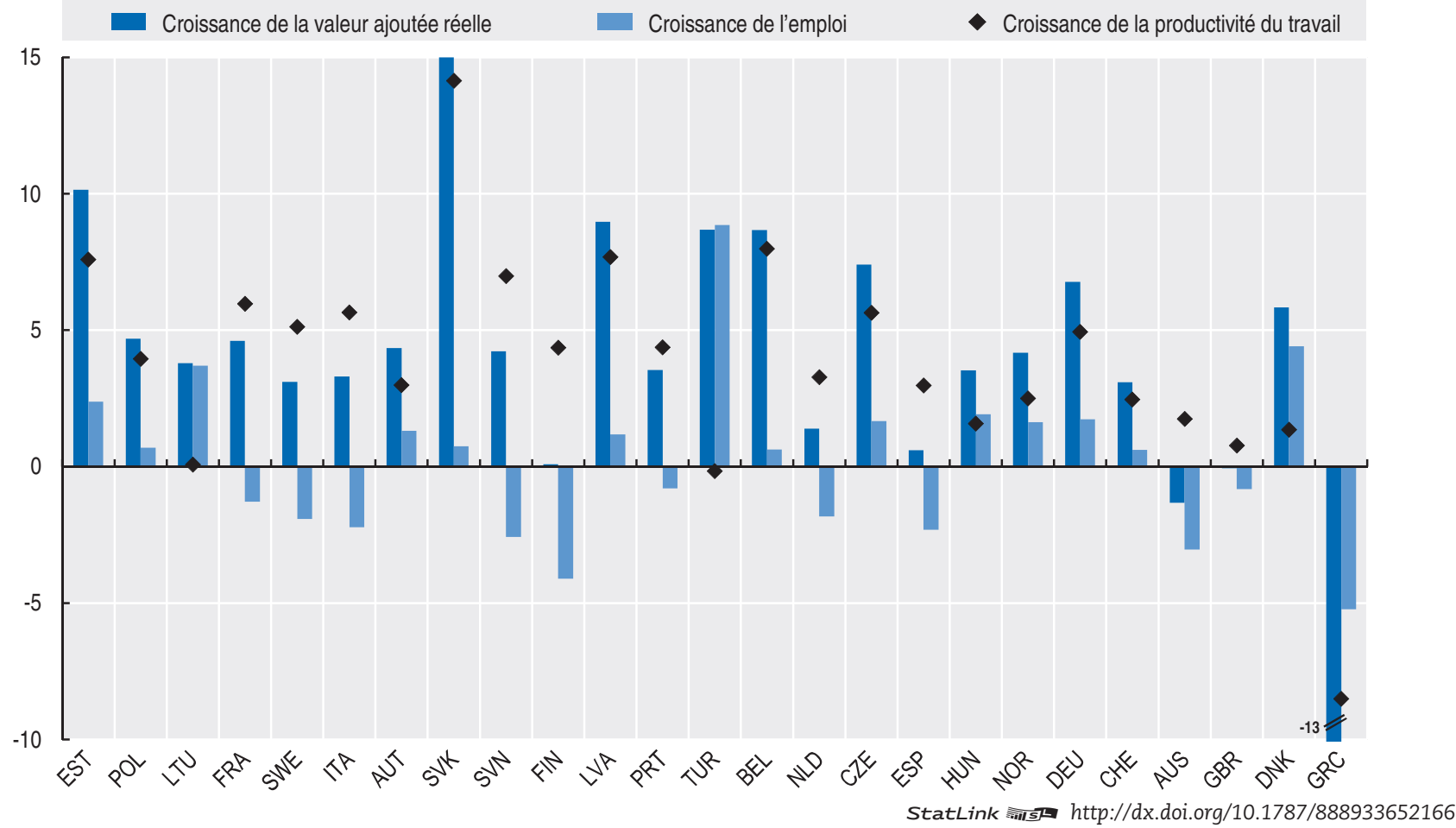




\section{PRODUCTIVITÉ PAR TAILLE D'ENTREPRISE}

Groissance de la productivité par taille d’entreprise

Graphique 3.6. Croissance de la valeur ajoutée réelle et de l'emploi par taille d'entreprise, services Taux annuel moyen, pourcentage, 2009-14

Petites et moyennes entreprises (1-249 salariés)
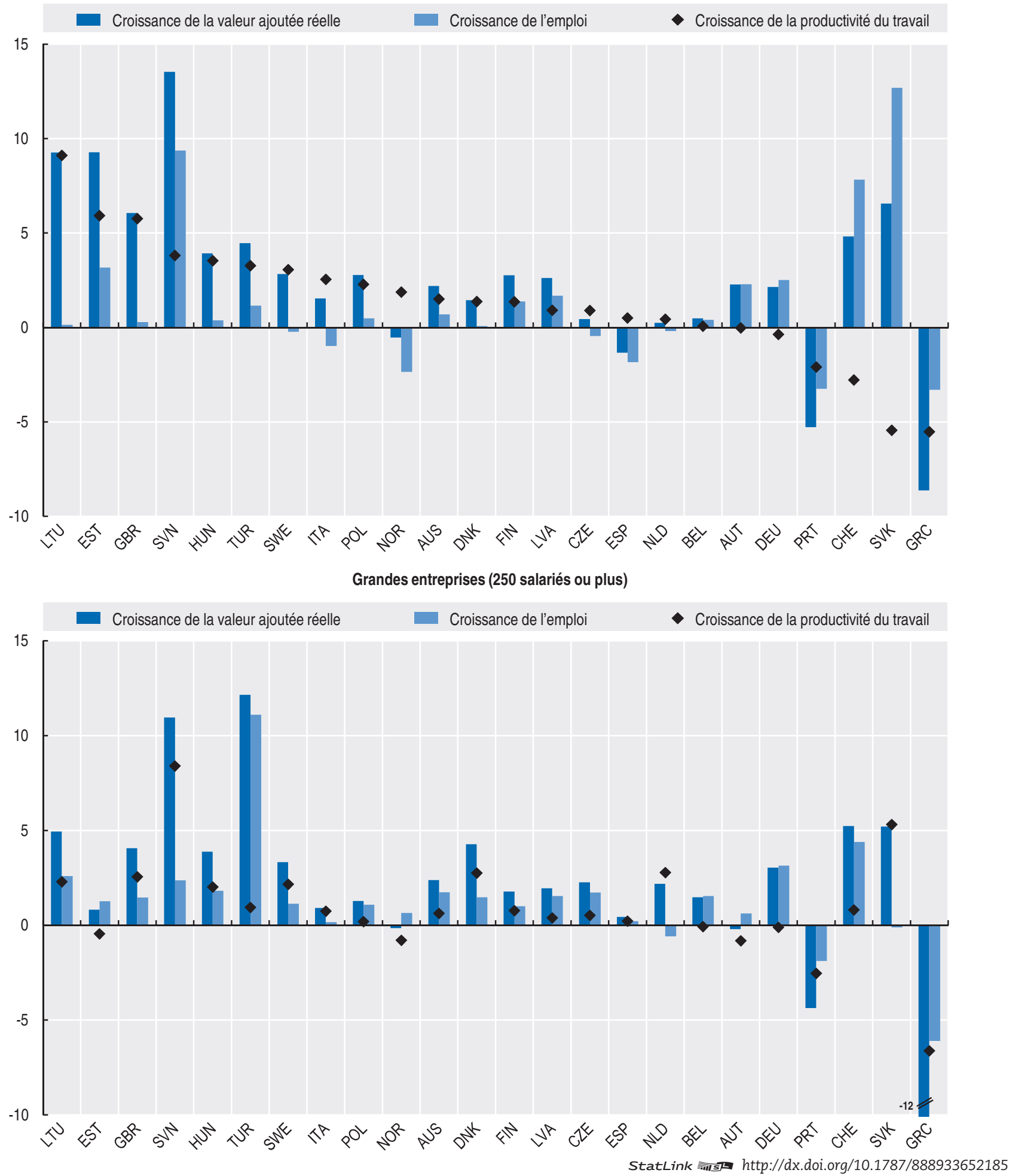


\section{À savoir}

- En règle générale, les salaires sont plus élevés dans les grandes entreprises manufacturières que dans les PME. En Allemagne, par exemple, les salariés des grandes entreprises gagnent plus de $50 \%$ de plus que ceux des entreprises de taille moyenne et plus du double de ceux des petites et microentreprises. Les écarts sont également importants en Belgique, en France et aux Pays-Bas, mais le sont nettement moins ailleurs, notamment en Finlande et en Slovénie.

- Les écarts de salaire entre entreprises coïncident généralement avec les écarts de productivité du travail. Les grandes entreprises du secteur manufacturier sont en moyenne plus productives et versent généralement des salaires plus élevés que les PME.

- Les écarts de rémunération moyenne du travail entre classes de taille d'entreprise se sont creusés dans le secteur manufacturier d'un certain nombre de pays, à savoir les économies d'Europe de l'Est membres de l'OCDE (exception faite de la Pologne), l'Estonie, la Lettonie, la Norvège et le Royaume-Uni.

\section{Pertinence}

L'inquiétude croît ces dernières années face à l'augmentation des inégalités de revenu. Dans certains pays, le ralentissement de la productivité des PME enregistré depuis la crise a creusé les écarts de productivité persistant dans nombre pays, ce qui limite les possibilités de réduire les inégalités.

\section{Définitions}

La rémunération des salariés comprend la rémunération totale, en espèces ou en nature, versée aux salariés en paiement du travail accompli par ces derniers au cours de la période de référence. Aucune rémunération n'est due pour le travail non rémunéré accompli sur une base volontaire, notamment le travail accompli par les membres d'un ménage au sein d'une entreprise non constituée en société et détenue par ce même ménage. La rémunération des salariés n'inclut pas les taxes sur les traitements et salaires dues par l'employeur. Elle comprend donc les traitements et salaires et autres cotisations patronales.

La rémunération du travail de l'ensemble des personnes occupées correspond à la somme des traitements et salaires versés à l'ensemble des personnes occupées et autres cotisations patronales.

La rémunération moyenne par taille d'entreprise exprimée en monnaie nationale est convertie en USD à partir des parités de pouvoir d'achat (PPA) pour la consommation individuelle effective, en conséquence de quoi elle rend compte de la rémunération moyenne du travail par salarié $\mathrm{du}$ point de vue des travailleurs/consommateurs.

La productivité du travail est la valeur ajoutée brute aux prix courants par personne occupée. Pour une définition des " activités de fabrication ", se reporter au Guide de lecture.

Informations sur les données concernant Israël : http:// dx.doi.org/10.1787/888932315602.

\section{Comparabilité}

Les données sur la valeur ajoutée correspondent à la valeur ajoutée au coût des facteurs dans les pays de l'UE et aux prix de base pour les autres pays. Les estimations de la valeur ajoutée et de l'emploi présentées par taille d'entreprise sont établies à partir de la base de données de l'OCDE sur les statistiques structurelles et démographiques des entreprises et ne coïncident généralement pas avec les estimations produites selon le Système de comptabilité nationale. Ce dernier prévoit en effet un certain nombre d'ajustements destinés à prendre en considération des entreprises et activités potentiellement absentes des statistiques structurelles sur les entreprises, comme les très petites unités ou les travailleurs indépendants, ou à tenir compte de l'économie non observée.

De nombreuses PME sont des entreprises non constituées en société. Leurs propriétaires ne se versent pas de salaire, mais reçoivent une rémunération sous forme de revenu mixte (selon la définition du Système de comptabilité nationale), qui est un élément de la valeur ajoutée. Il est donc fort probable que les estimations uniquement fondées sur la rémunération des salariés sous-estiment la contribution relative du travail dans les PME, par comparaison avec les estimations relatives aux grandes entreprises.

Pour l'Australie et Israël, les données présentées font référence à la rémunération de l'ensemble des personnes occupées.

La comparabilité des classes de taille, secteurs et pays peut pâtir des disparités de la part de l'emploi à temps partiel dans l'emploi total. C'est pourquoi la mesure privilégiée du facteur travail, dans l'analyse de la productivité, est le nombre total d'heures travaillées plutôt que l'effectif, mais on ne dispose généralement pas de ventilation de ces données par taille d'entreprise. Mesurer la rémunération des salariés par heure travaillée rend mieux compte des disparités internationales en matière d'emploi à temps partiel, mais cela n'est possible que pour un nombre limité de pays.

Certains pays utilisent des conventions différentes en matière de ventilation par classe de taille : pour l'Australie, " 1-9 » correspond à « 1-19 ", « 20-49 » à 《 20-199 » et « 250+ " à 《 200+ "; pour le Mexique, «1-9 » correspond à «1-10 ", « 10-19 » à « 11-20 ", « 20-49 » à « 21-50 », « 50-249 » à « 51- 250 » et « 250+ » à « 251+ ».

L'unité statistique utilisée pour le Mexique n'est pas l'entreprise, mais l'établissement. Pour le Royaume-Uni, les données présentées n'incluent pas les quelque 2.6 millions de petites entreprises non enregistrées, c'est-à-dire des entreprises n'atteignant pas le seuil prévu pour le régime de la taxe sur la valeur ajoutée et/ou celui de la retenue de l'impôt à la source (pour les entreprises employant des salariés).

\section{Sources}

OCDE, Statistiques structurelles et démographiques des entreprises (SDBS) (base de données), http://dx.doi.org/10.1787/sdbs-data-fr.

\section{Pour en savoir plus}

OCDE (2017), OECD Compendium of Productivity Indicators 2017, Éditions OCDE, Paris, http://dx.doi.org/10.1787/pdtuy-2017-en

OCDE (2017), Perspectives de l'emploi de l'OCDE 2017, Éditions OCDE, Paris, http://dx.doi.org/10.1787/empl_outlook-2017-fr

OCDE (2001), Mesurer la productivité - Manuel de l'OCDE Mesurer la croissance de la productivité par secteur et pour l'ensemble de l'économie, Éditions OCDE, Paris, http://dx.doi. org/10.1787/9789264294516-fr. 
Graphique 3.7. Rémunération par salarié et par taille d'entreprise, activités de fabrication Milliers d'USD, PPA pour la consommation individuelle effective, 2014, ou dernière année disponible

$=1-9 \quad \times 20-49 \quad \Delta 50-249 \quad-250+$

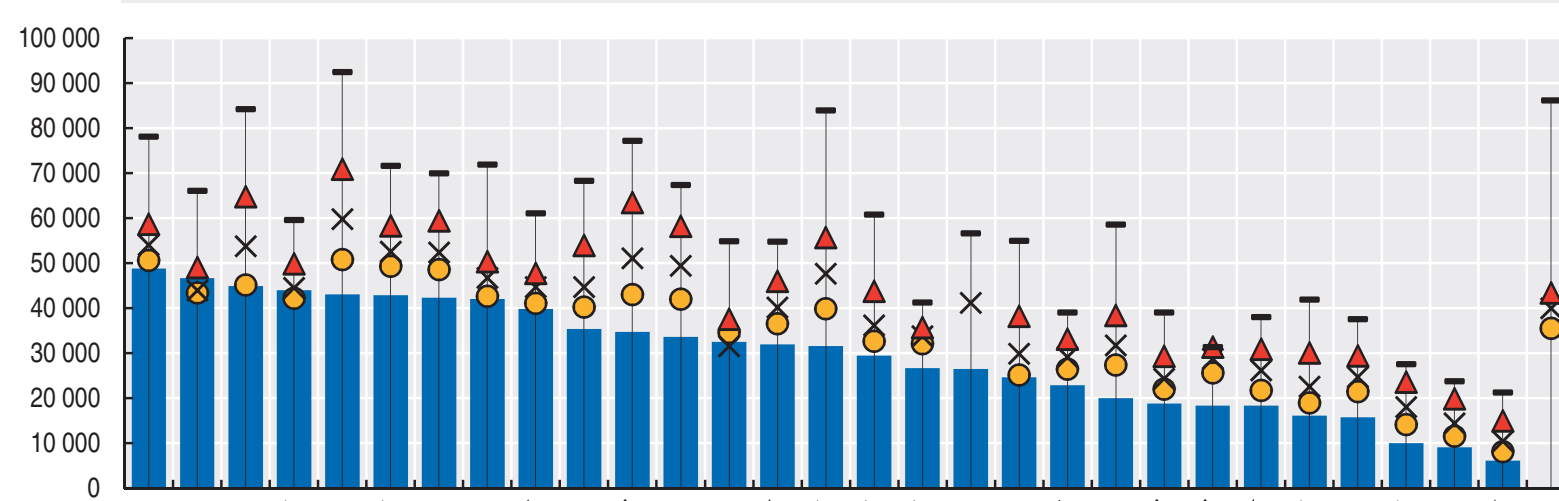

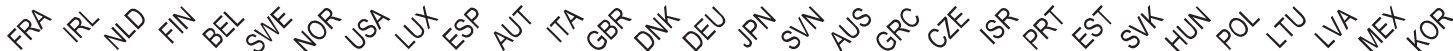

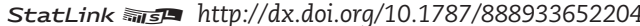

Graphique 3.8. Productivité du travail et rémunération par salarié et par taille d'entreprise, activités de fabrication Valeur ajoutée par salarié (VAPS) et rémunération par salarié (RPS), indice 250+ =100, 2014, ou dernière année disponible
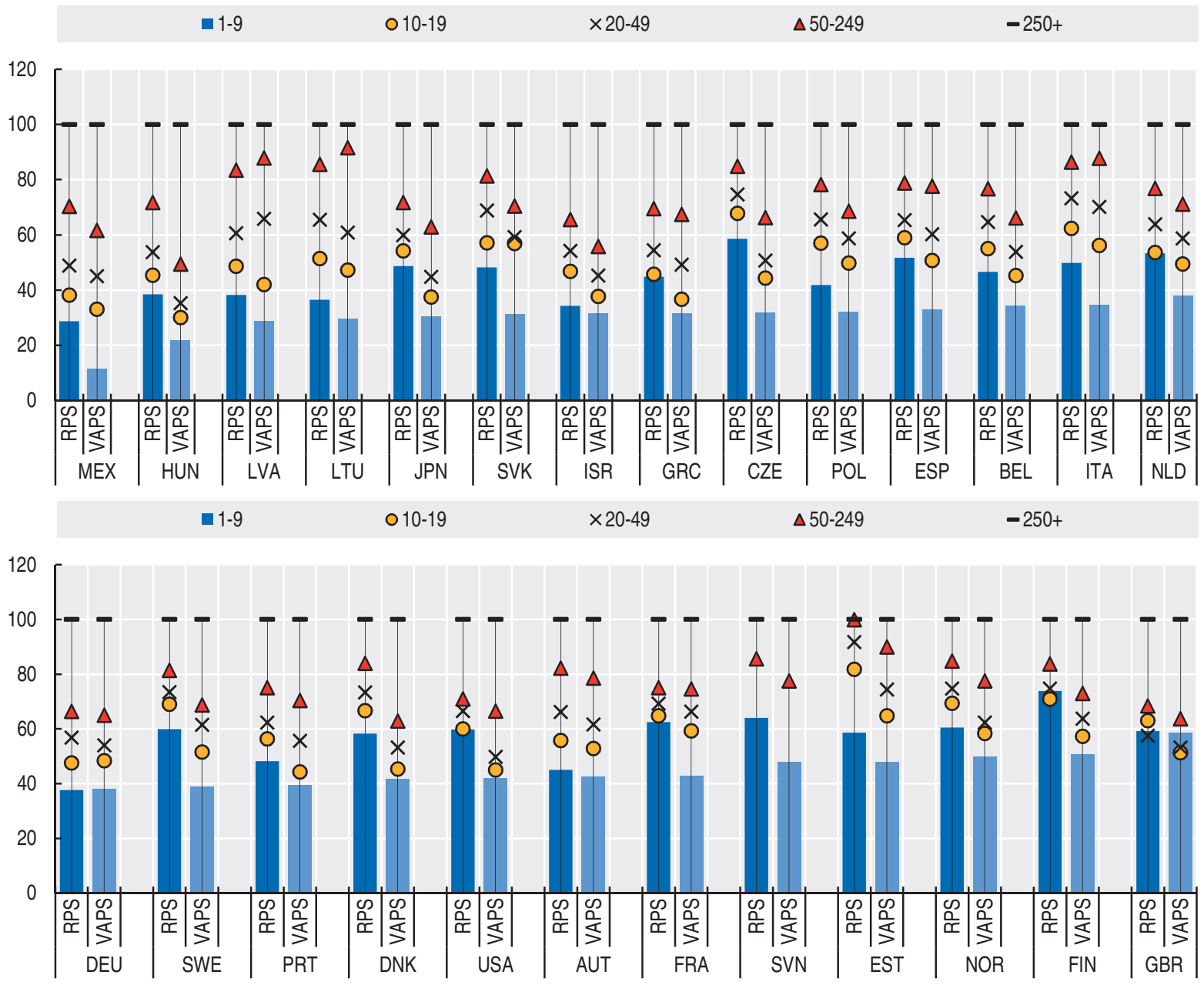


\section{PRODUCTIVITÉ PAR TAILLE D’ENTREPRISE}

\section{Productivité et écarts de salaire entre les entreprises}

Graphique 3.9a. Croissance de la rémunération moyenne par salarié et par taille d'entreprise, activités de fabrication

Pays dans lesquels la croissance des salaires est similaire pour toutes les classes de taille d'entreprise, prix courants, indice $2008=100$
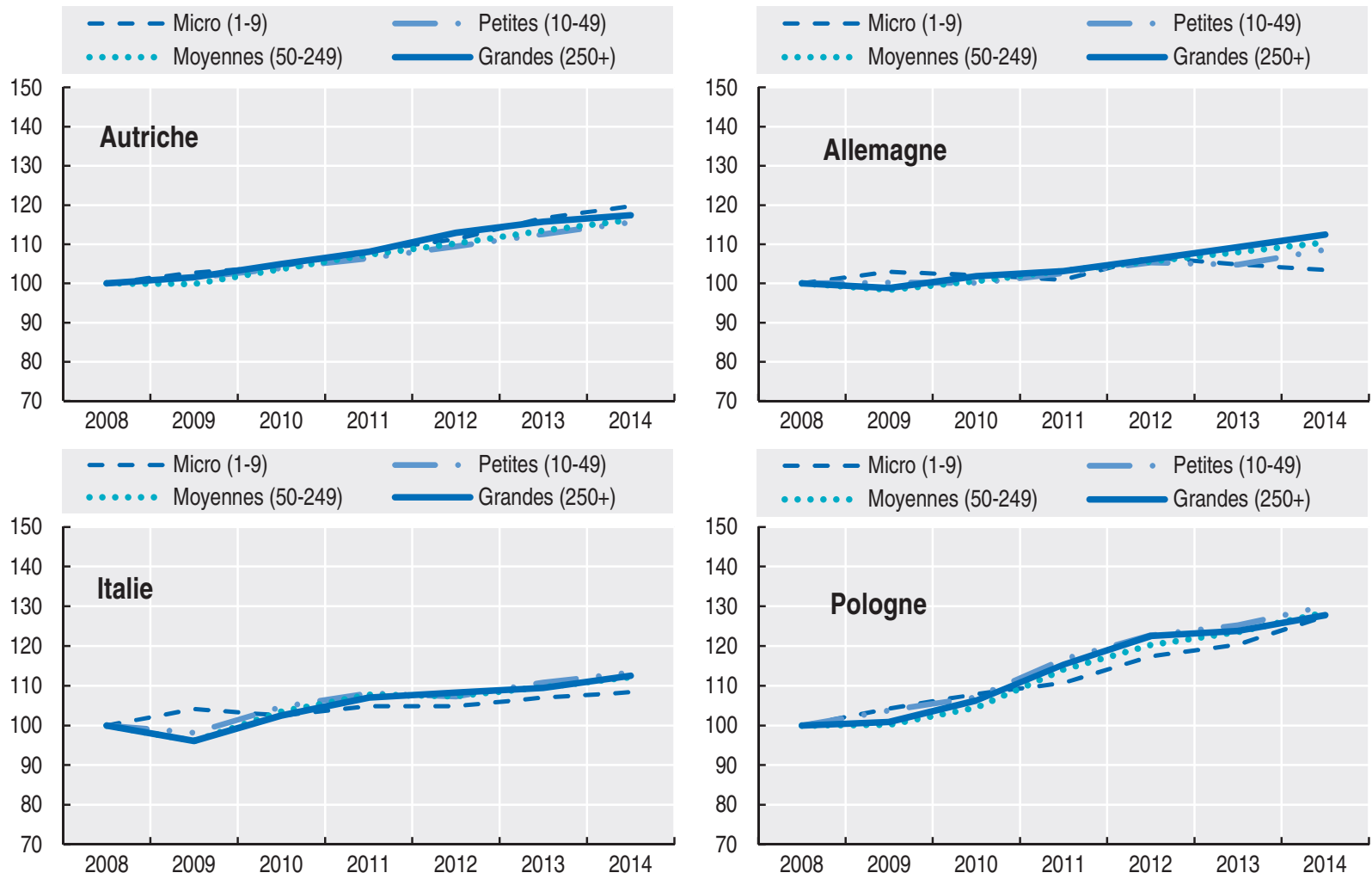

$\begin{array}{ll}-\ldots \text { Micro }(1-9) & \text { Petites }(10-49) \\ \ldots . . . \text { Moyennes }(50-249) \quad \text { Grandes }(250+)\end{array}$

150
140
130
120
110
100
90
80
70
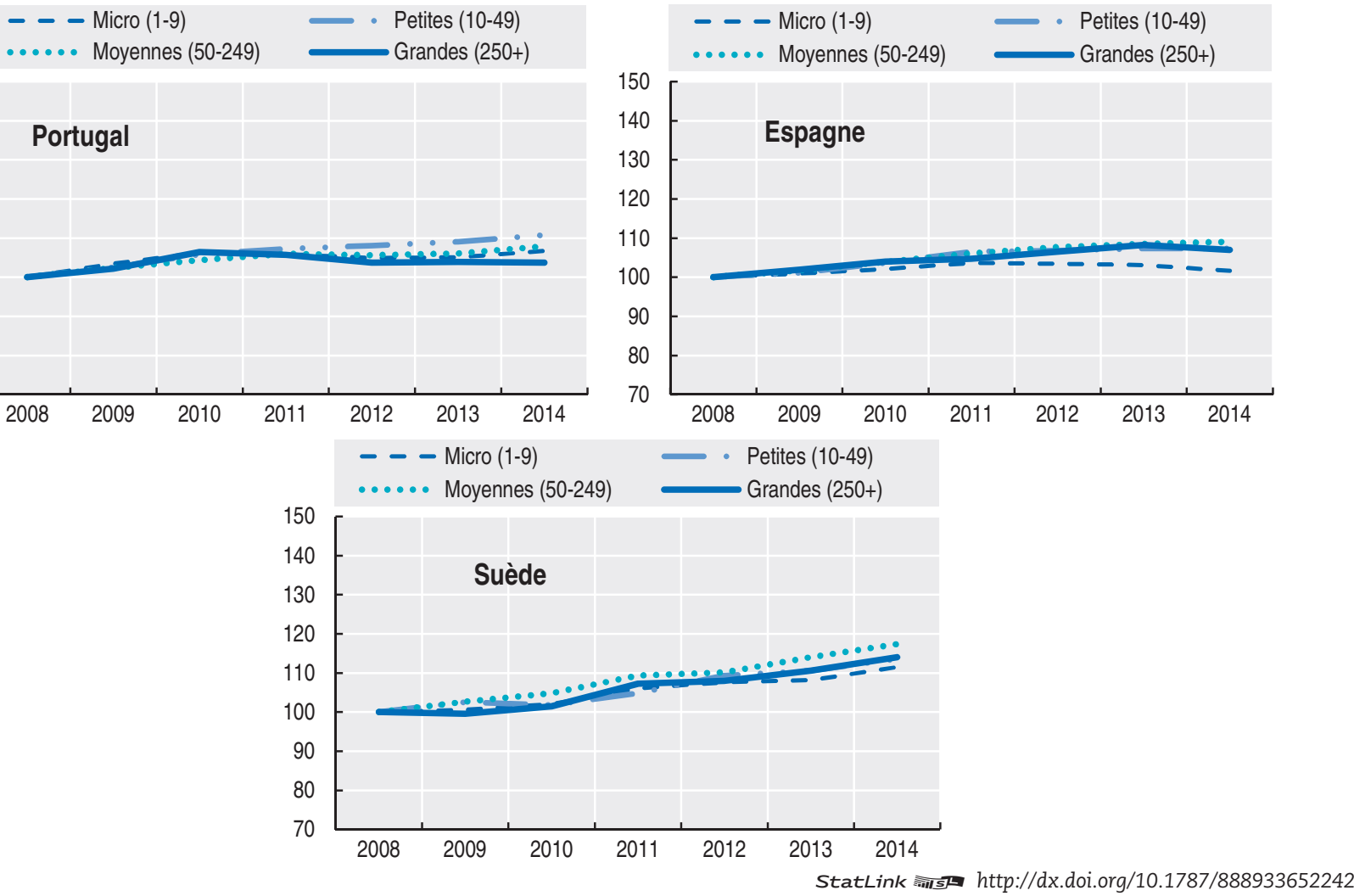

- - Micro (1-9)

...... Moyennes (50-249)

Grandes (250+) 


\section{PRODUCTIVITÉ PAR TAILLE D'ENTREPRISE}

Productivité et écarts de salaire entre les entreprises

Graphique 3.9b. Croissance de la rémunération moyenne par salarié et par taille d'entreprise, activités de fabrication (suite)

Pays dans lesquels les écarts de salaire se creusent entre les PME et les grandes entreprises, prix courants, indice $2008=100$
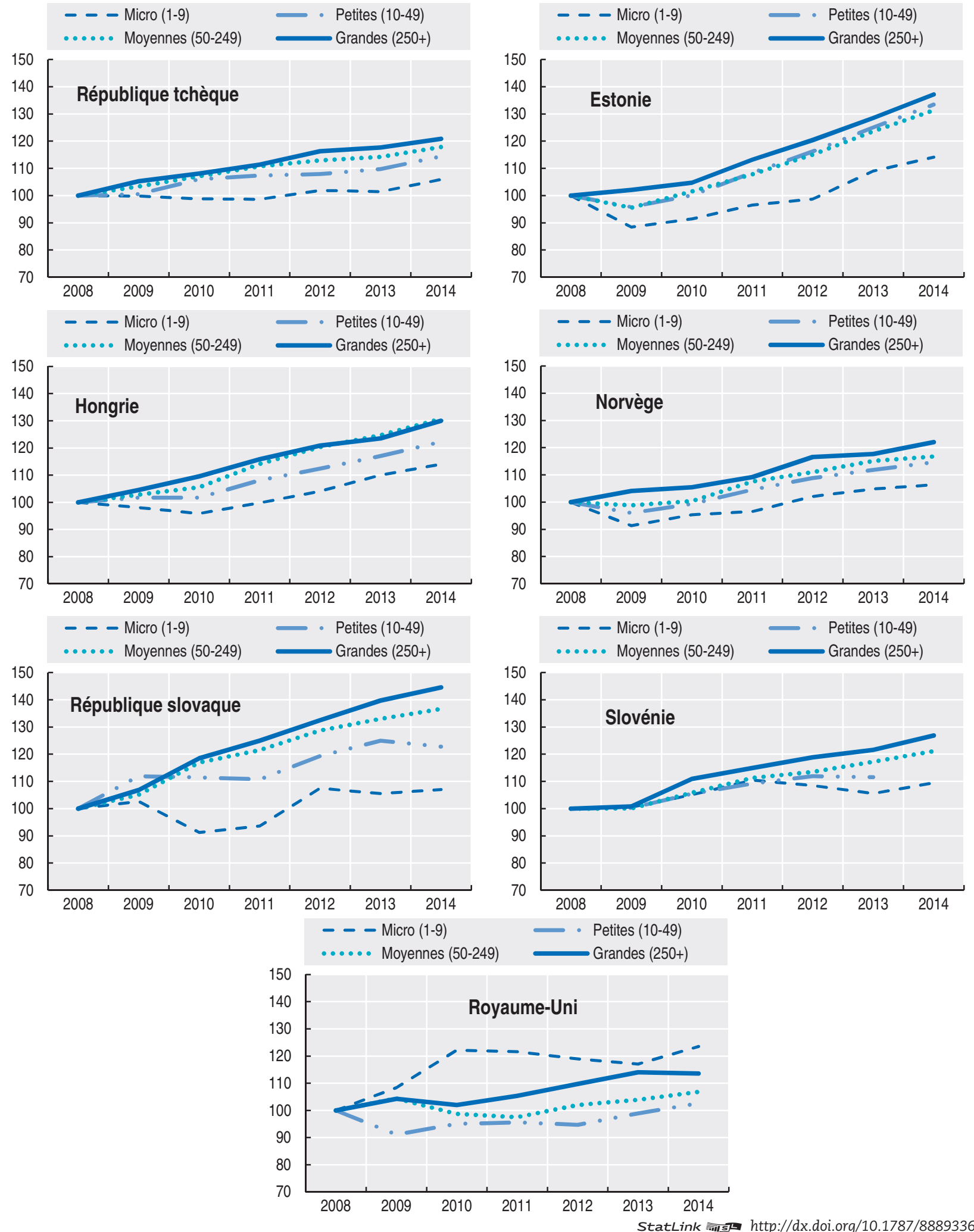

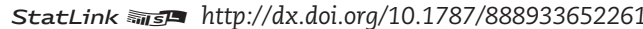





\title{
4. DYNAMISME DES ENTREPRISES ET CRÉATION D'EMPLOIS
}

\author{
Naissances d'entreprises \\ Décès d'entreprises \\ Renouvellement des entreprises \\ Jeunes entreprises \\ Entreprises à forte croissance
}




\section{À savoir}

- Si le nombre des naissances d'entreprises sans salarié est généralement plus élevé que celui des naissances d'entreprises employant des salariés, cette première catégorie représentait dans la plupart des économies de l'OCDE une proportion plus faible des créations d'emplois totales en 2014.

- La majorité des nouvelles entreprises employant des salariés dans les économies de l'OCDE comptent entre un et quatre salariés. Le nombre moyen de personnes qui y sont employées est généralement plus élevé dans l'industrie que dans les services, du fait des économies d'échelle.

- La taille moyenne des nouvelles entreprises employant des salariés est globalement similaire dans tous les pays dans le secteur des services. Des différences plus marquées existent dans l'industrie. La taille moyenne des nouvelles entreprises créées dans l'industrie aux États-Unis était trois fois supérieure à celles créées en Italie en 2014.

- Dans pratiquement tous les pays, les taux de naissance sont plus élevés dans les secteurs de la construction et des services, en particulier dans les activités d'hébergement et de restauration, et dans les activités professionnelles, scientifiques et techniques, que dans l'industrie, une différence qui s'explique en partie par des coûts d'entrée en capital fixe moins élevés.

\section{Pertinence}

La naissance de nouvelles entreprises est un indicateur clé du dynamisme des entreprises. Elle renseigne sur une dimension importante de l'entrepreneuriat dans un pays, à savoir la capacité de créer une activité entièrement nouvelle. Les nouvelles entreprises sont considérées comme des moteurs essentiels de la croissance en raison de leur contribution disproportionnée à la création globale d'emplois et de l'effet stimulateur sur la productivité associé au rythme soutenu de leurs entrées et sorties.

\section{Définitions}

La naissance d'une entreprise employant des salariés désigne la naissance d'une entreprise comptant au moins un salarié. Cette population comprend d'une part les entreprises " nouvellement nées ", c'est-à-dire les entités nouvelles déclarant au moins un salarié au cours de leur première année d'existence ; et d'autre part celles qui, alors qu'elles existaient mais n'employaient pas de salarié avant l'année considérée, ont déclaré au moins un salarié durant l'année en cours, à savoir leur année de naissance. Les naissances d'entreprises employant des salariés n'incluent pas les entrées survenues dans la population à la suite de fusions, dissolutions, scissions ou restructurations au sein d'un groupe d'entreprises. De même, ne sont pas comprises les entrées dans une sous-population qui ne résultent que d'un changement d'activité.

Le taux de naissance des entreprises employant des salariés correspond au nombre de naissances d'entreprises employant des salariés exprimé en pourcentage de la population des entreprises actives comptant au moins un salarié. Le taux de naissance dans un secteur $\mathrm{x}$ est mesuré en pourcentage des entreprises actives du secteur x comptant au moins un salarié.

La naissance d'une entreprise sans salarié désigne la naissance d'une entreprise sans salarié. Le taux de naissance des entreprises sans salarié correspond au nombre de naissances d'entreprises sans salarié en pourcentage de la population d'entreprises actives sans salarié.
L'effectif moyen dans les entreprises créées employant des salariés s'obtient en divisant le nombre de personnes occupées dans des entreprises créées en t par le nombre d'entreprises créées en $t$.

Informations sur les données concernant Israël : http:// dx.doi.org/10.1787/888932315602.

\section{Comparabilité}

Les indicateurs concernant les " entreprises employant des salariés » dépendent moins du champ couvert par les registres d'entreprises que ceux couvrant toutes les entreprises. Dans de nombreux pays, les principales sources de données utilisées par ces registres sont les registres administratifs fiscaux et de l'emploi, qui amènent souvent à ne prendre en compte que les entreprises affichant un certain seuil de chiffre d'affaires et/ou d'effectif. Une économie dans laquelle les seuils sont relativement élevés devrait donc logiquement afficher des statistiques de naissance inférieures à celles des économies de même type dotées de seuils plus bas. En outre, les seuils, notamment les seuils de nature monétaire, peuvent évoluer dans le temps en fonction par exemple de l'inflation et de la politique budgétaire. L'utilisation du seuil d'un salarié améliore la comparabilité car elle permet d'exclure les très petites unités, qui sont les plus sujettes aux variations de seuil.

Le concept de naissance d'entreprises employant des salariés pose toutefois des problèmes. De nombreux pays ont une population non négligeable de travailleurs indépendants propriétaires d'une entreprise qui n'emploie aucun salarié. Si un pays met en place des incitations pour que les travailleurs indépendants se salarient dans leur propre entreprise, le nombre total de naissances d'entreprises employant des salariés augmentera, ce qui risque de fausser les comparaisons chronologiques et internationales, même si le changement est minime d'un point de vue économique et entrepreneurial.

Les données pour le Canada, les États-Unis et Israël font référence au nombre d'employés. Au graphique 4.1, les données font uniquement référence à la population des entreprises employant des salariés pour le Brésil, le Canada, les États-Unis, Israël et la Nouvelle-Zélande.

Pour l'Australie, la Corée et le Mexique, les naissances d'entreprises et les indicateurs connexes ne tiennent pas compte des entreprises sans salarié qui sont devenues des entreprises employant au moins un salarié. Autrement dit, lorsqu'une entreprise sans salarié devient une entreprise employant des salariés, ce changement de statut n'est pas considéré comme une "naissance d'entreprise employant des salariés ".

\section{Source}

OCDE, Statistiques structurelles et démographiques des entreprises (SDBS) (base de données), http://dx.doi.org/10.1787/sdbs-data-fr.

Counts of Australian Businesses, including Entries and Exits. 8165.0, http://dx.doi.org/10.1787/sdbs-data-fr.

\section{Pour en savoir plus}

Ahmad, N. (2006), " A Proposed Framework for Business Demography Statistics ", OECD Statistics Working Papers, vol. 2006, n³, Éditions OCDE, Paris, http://dx.doi.org/10.1787/ 145777872685.

OCDE/Eurostat (2008), Eurostat-OECD Manual on Business Demography Statistics, Éditions OCDE, Paris, http://dx.doi. org/10.1787/9789264041882-en. 
Graphique 4.1. Naissances des entreprises sans salarié et employant des salariés, créations d'emplois, secteur marchand

En pourcentage, 2014 ou dernière année disponible

$\square$ Part des créations d'emplois due aux naissances d'entreprises employant des salariés dans l'emploi total - Part des naissances d'entreprises employant des salariés dans le nombre total des entreprises

- Part des naissances d'entreprises sans salarié dans le nombre total des entreprises

o Part des créations d'emplois due aux naissances d'entreprises sans salarié dans l'emploi total

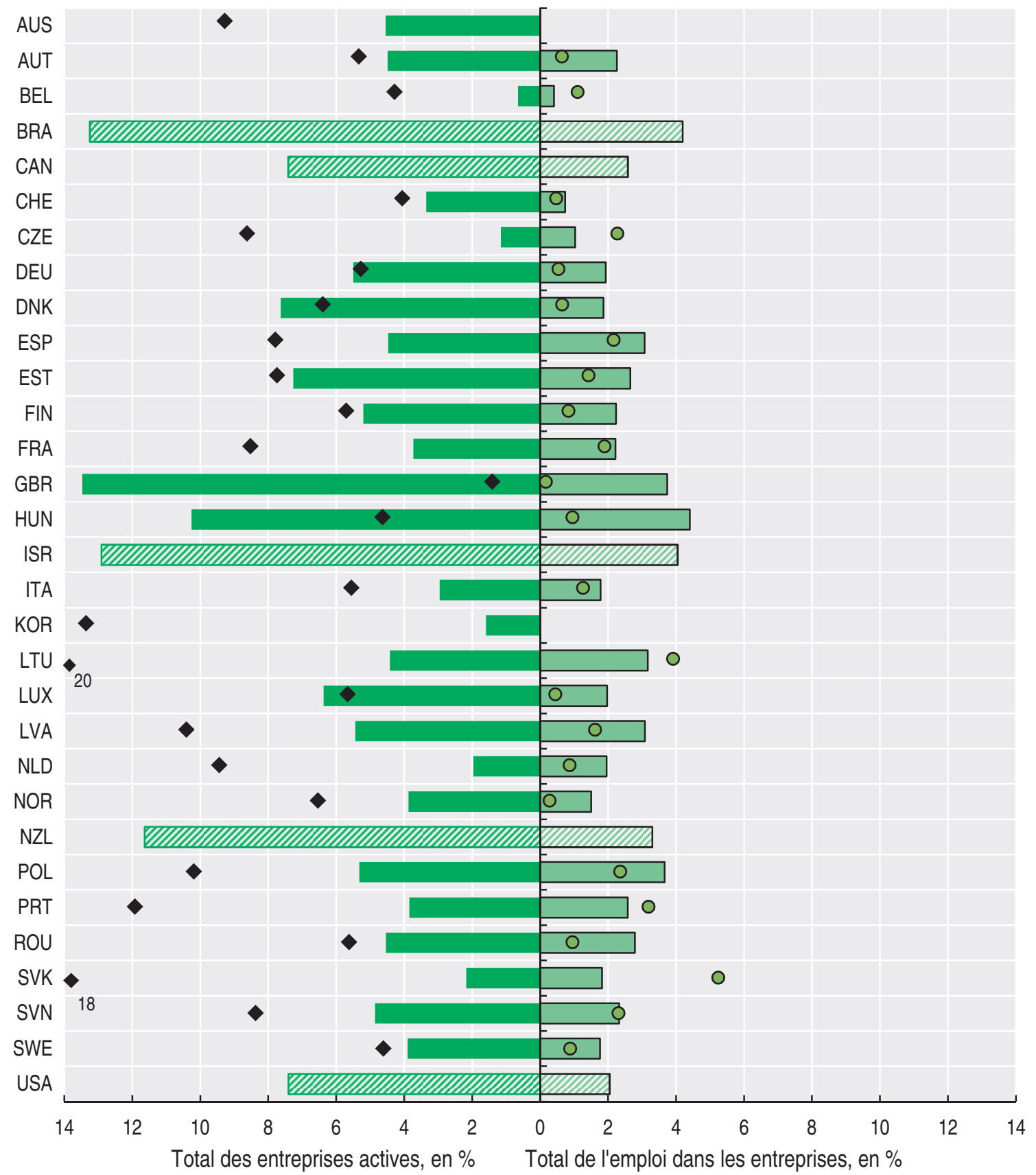

StatLink 全IS http://dx.doi.org/10.1787/888933652280 


\section{DYNAMISME DES ENTREPRISES ET CRÉATION D’EMPLOIS}

\section{Naissances d'entreprises}

Graphique 4.2. Nombre de naissances d'entreprises employant des salariés et sans salarié, secteur marchand En milliers d'entreprises, 2014 ou dernière année disponible

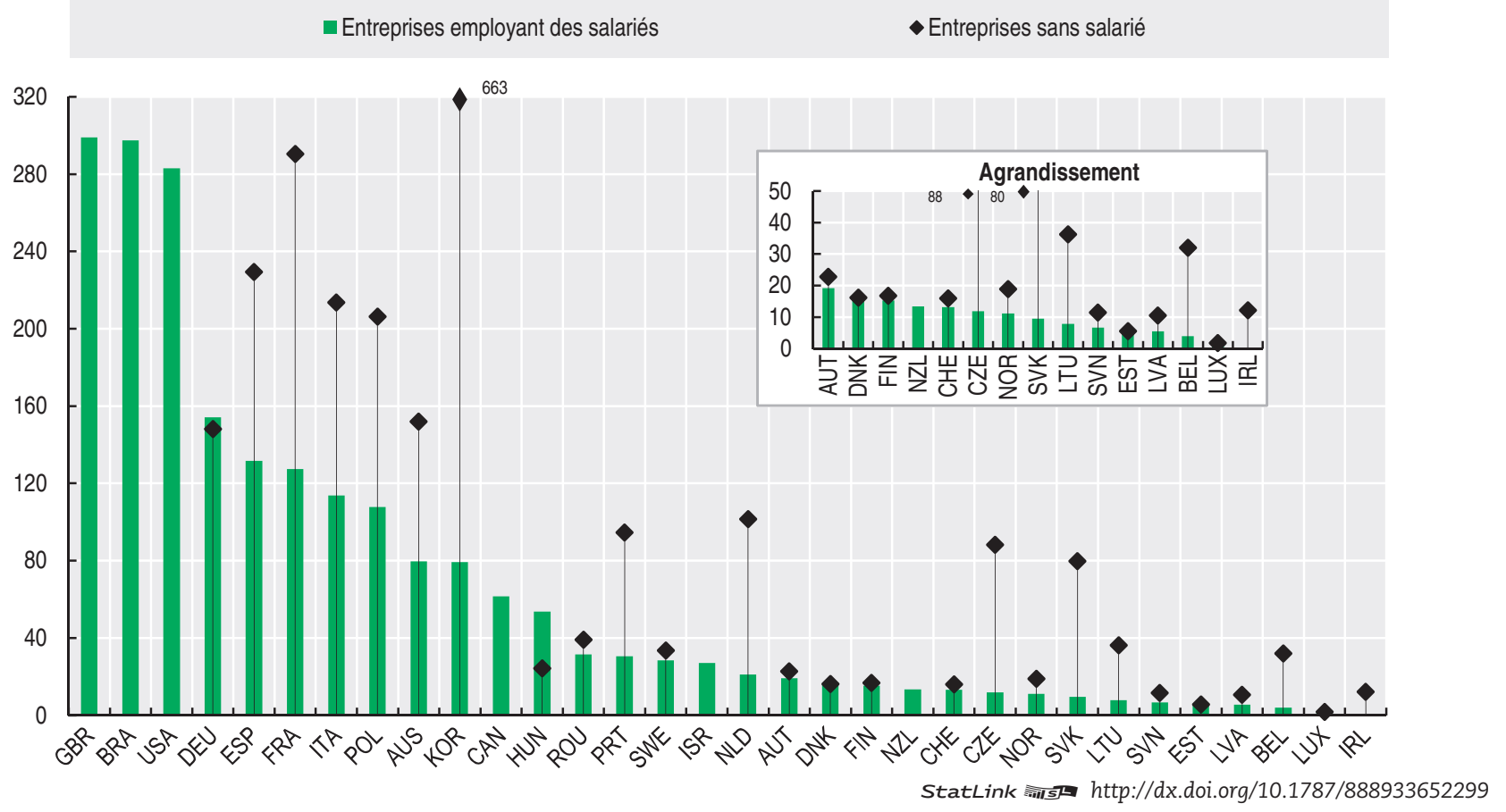

Graphique 4.3. Taux de naissance des entreprises employant des salariés, secteur marchand

Nombre des naissances d'entreprises employant des salariés en pourcentage des entreprises actives employant des salariés, 2014 ou dernière année disponible

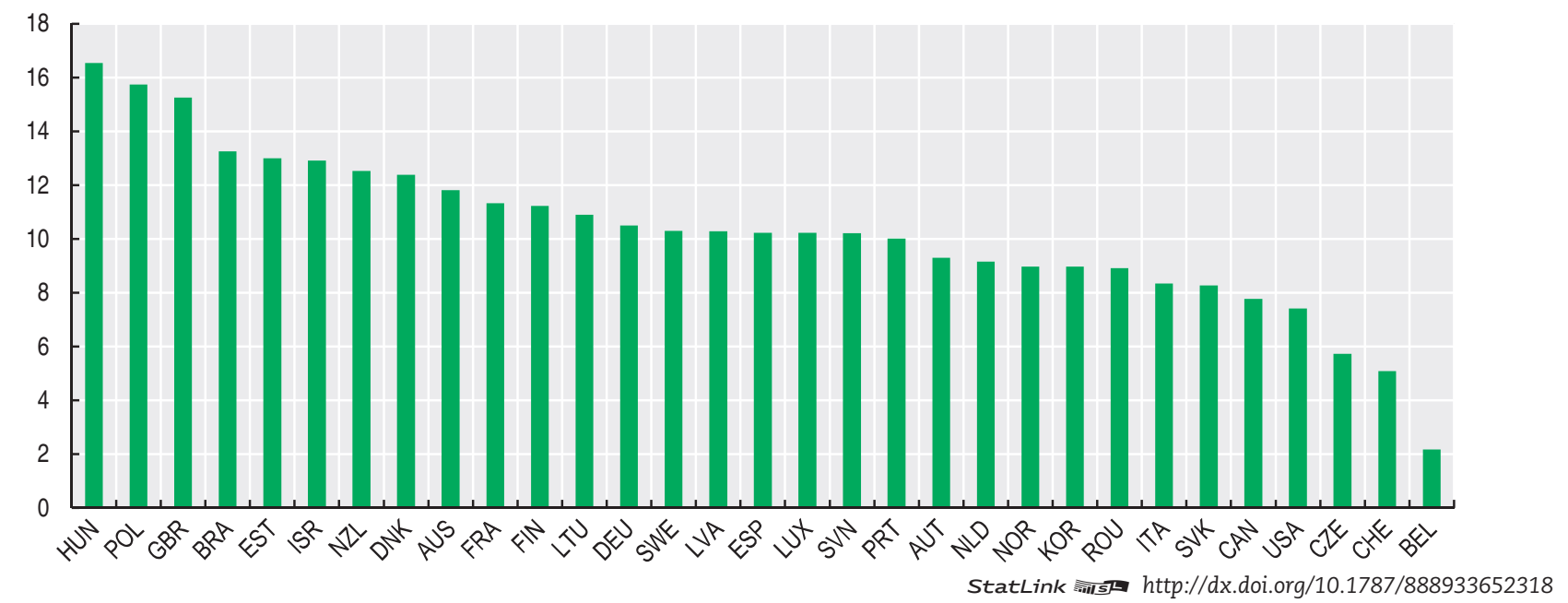


Graphique 4.4. Taux de naissance des entreprises employant des salariés, principaux secteurs

En pourcentage des entreprises actives employant des salariés dans chaque secteur, 2014 ou dernière année disponible

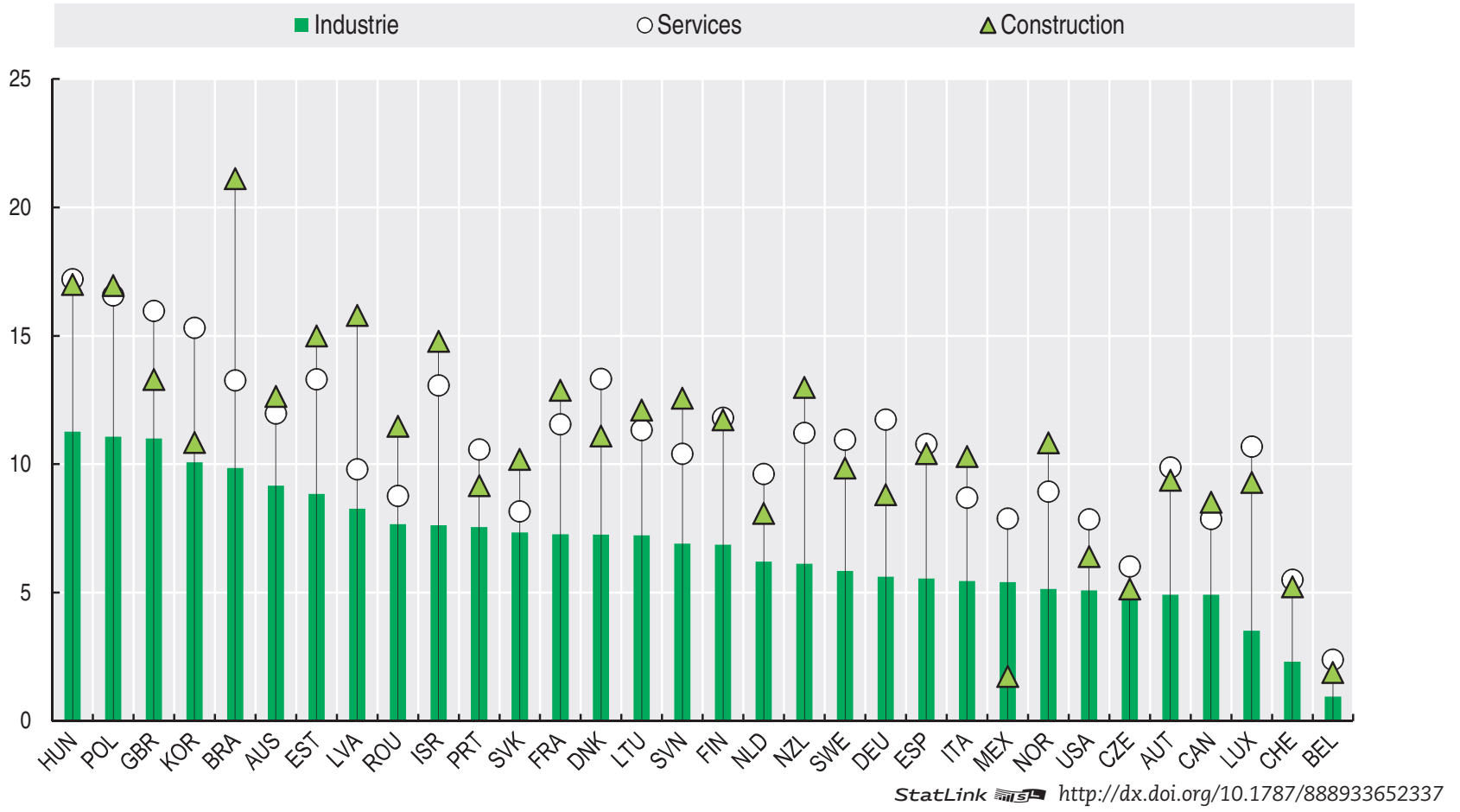

Graphique 4.5. Créations d'emplois dues aux naissances d'entreprises employant des salariés, secteur marchand En milliers de personnes employées, 2014 ou dernière année disponible

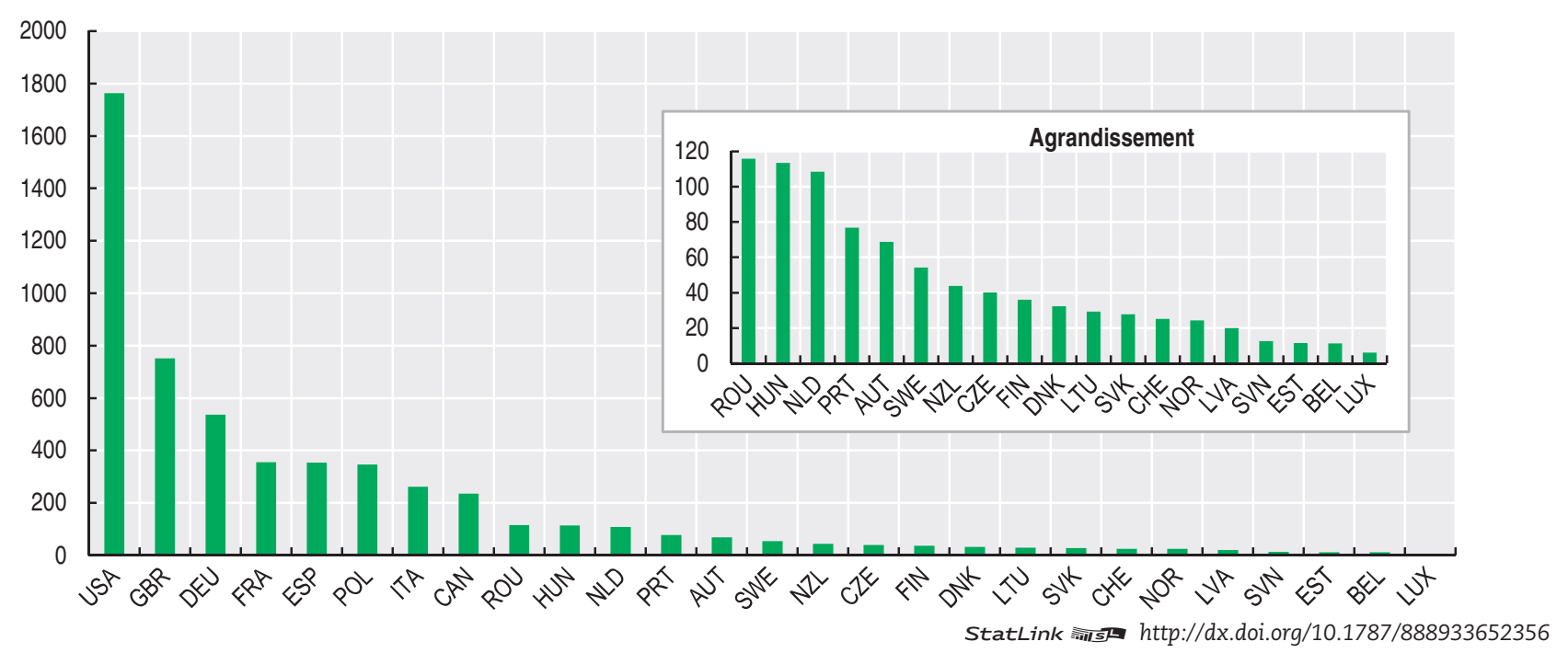




\section{DYNAMISME DES ENTREPRISES ET CRÉATION D'EMPLOIS}

\section{Naissances d'entreprises}

Graphique 4.6. Créations d'emplois dues aux naissances d'entreprises employant des salariés, ventilation par activité économique

En pourcentage des naissances totales, 2014 ou dernière année disponible

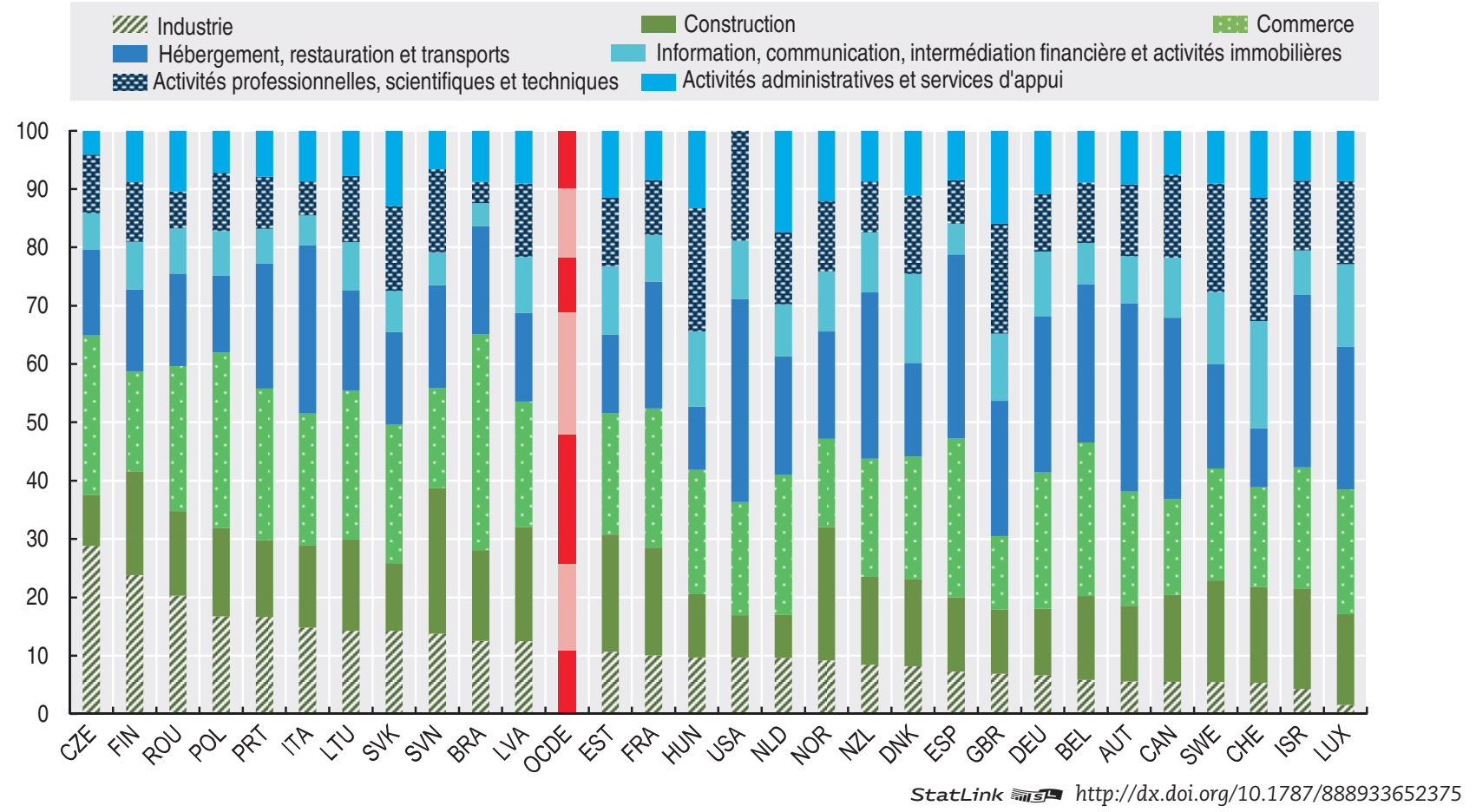

Graphique 4.7. Créations d'emplois dues aux naissances d'entreprises employant des salariés, par activité économique

En pourcentage de l'emploi total dans chaque activité économique, 2014 ou dernière année disponible

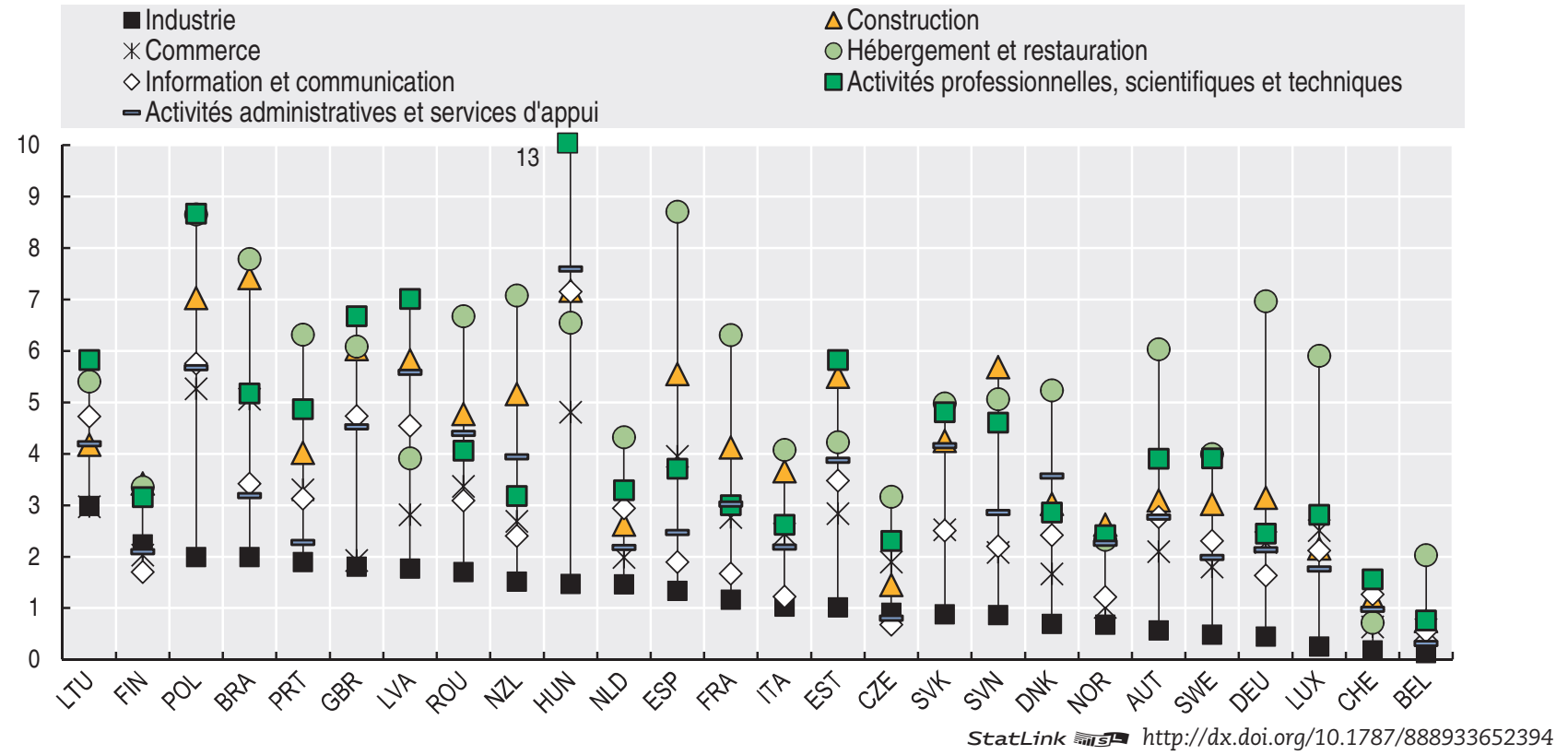


Graphique 4.8. Naissances d'entreprises employant des salariés, par taille d'entreprise et principaux secteurs Pourcentage (proportion des classes de taille) et nombre de personnes employées par entreprise nouvellement créée (effectif moyen), 2014 ou dernière année disponible

\section{Industrie}

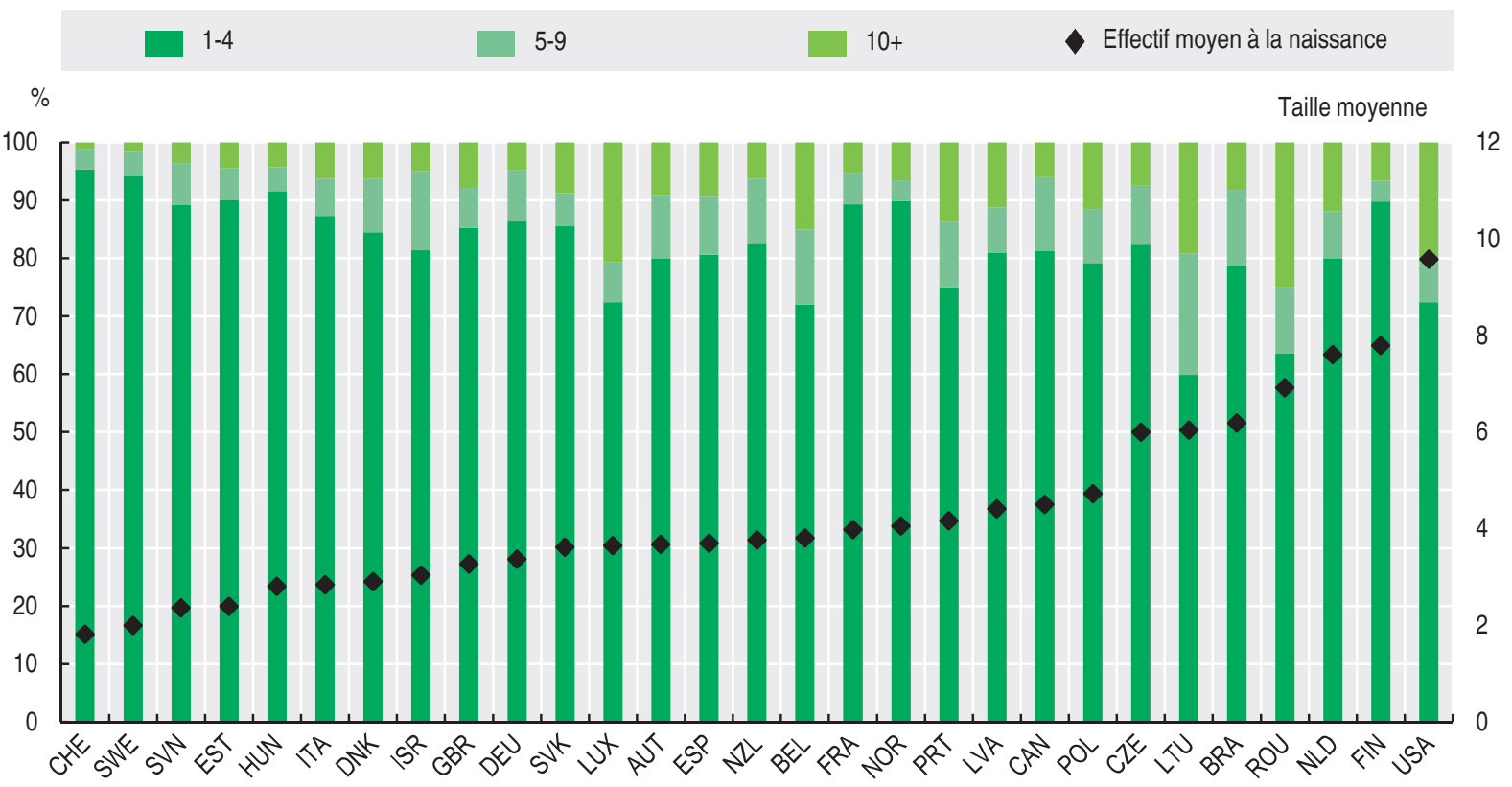

Services

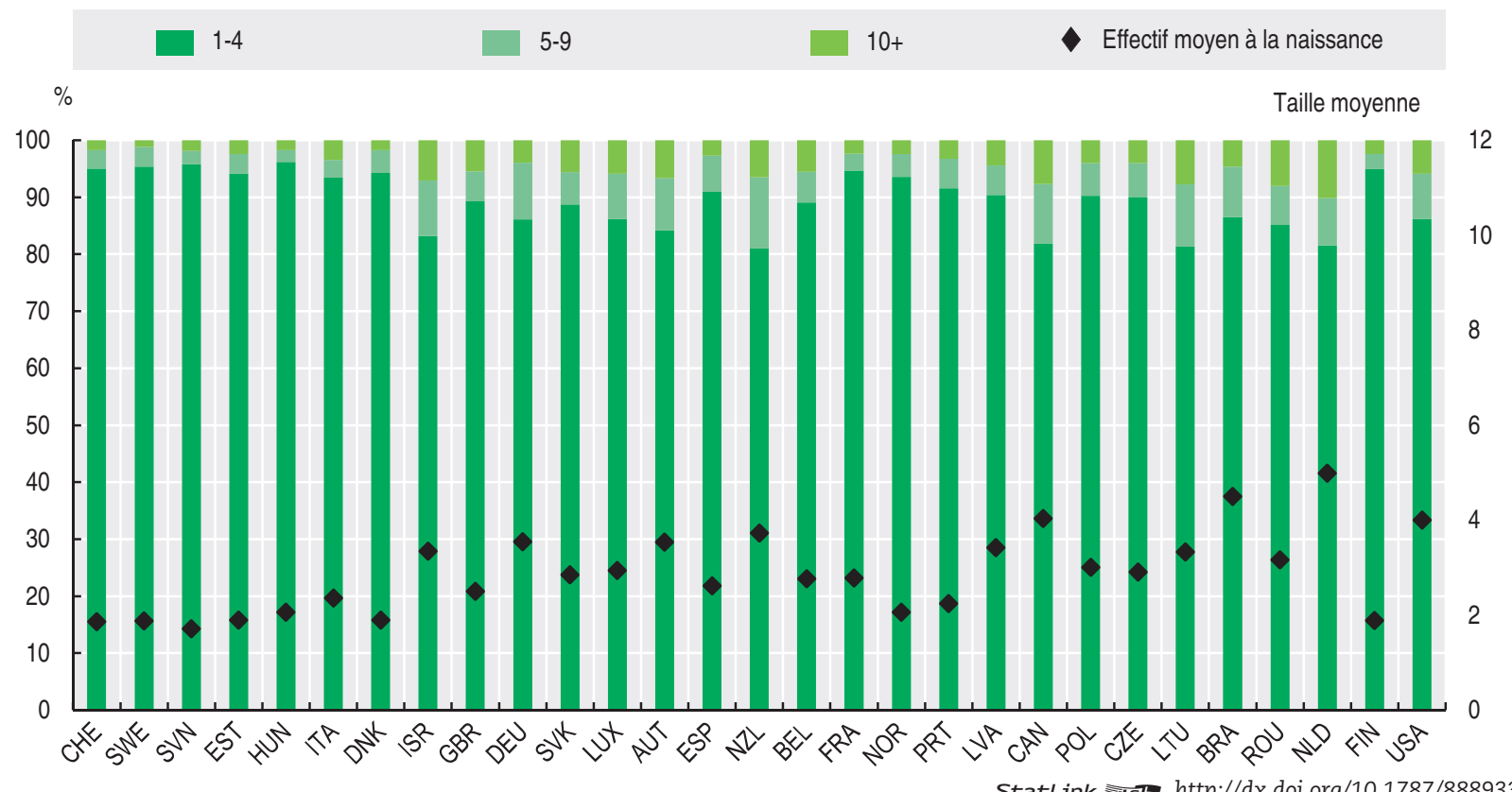

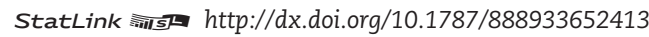




\section{À savoir}

- Les taux de décès des entreprises sans salarié sont généralement plus élevés que ceux des entreprises employant des salariés, signe du statut souvent précaire de la première catégorie. Les décès d'entreprises employant des salariés représentent toutefois davantage de destructions d'emplois que ceux des entreprises sans salarié, et ce, dans la majorité des pays.

- Quel que soit le pays, les décès d'entreprises employant des salariés dans le secteur des services touchent globalement des entreprises de taille moyenne similaire. Des différences plus marquées existent dans l'industrie, où la taille moyenne des entreprises employant des salariés au moment de leur décès était, aux États-Unis (35 salariés), dix fois supérieure à celle des entreprises italiennes en 2014.

- Dans tous les pays, le taux de décès des entreprises employant des salariés dans les secteurs de la construction et des services est systématiquement plus élevé que dans l'industrie.

\section{Pertinence}

Le décès des entreprises fait partie intégrante du phénomène entrepreneurial. L'analyse du taux de sortie du marché des entreprises dans le temps et dans les différents pays permet de mieux comprendre le processus de "destruction créatrice » et limpact des cycles économiques sur lientrepreneuriat.

\section{Définitions}

Le décès d'une entreprise employant des salariés correspond soit au décès d'une entreprise comptant au moins un salarié au cours de l'année concernée, soit au passage d'une entreprise sous le seuil d'un salarié pendant au moins deux ans. Les décès n'incluent pas les sorties de la population dues à des fusions, absorptions, dissolutions ou restructurations au sein d'un groupe d'entreprises. De même, les sorties d'une sous-population ne résultant que d'un changement d'activité ne sont pas incluses.

Le décès d'une entreprise sans salarié se produit lorsqu'une entreprise quitte la population des entreprises sans salarié, soit au moment de son décès, soit lorsqu'elle augmente ses effectifs.

Le taux de décès des entreprises employant des salariés correspond au nombre de décès d'entreprises employant des salariés, exprimé en pourcentage de la population d'entreprises actives comptant au moins un salarié. Le taux de décès dans un secteur $\mathrm{x}$ est exprimé en pourcentage des entreprises actives du secteur $\mathrm{x}$ employant au moins un salarié.

Le taux de décès des entreprises sans salarié correspond au nombre de décès d'entreprises sans salarié, exprimé en pourcentage de la population d'entreprises actives sans salarié.

L'effectif moyen dans les entreprises détruites s'obtient en divisant le nombre de personnes occupées dans des entreprises détruites en t par le nombre d'entreprises détruites en $t$.

Informations sur les données concernant Israël : http:// dx.doi.org/10.1787/888932315602.

\section{Comparabilité}

Les indicateurs concernant les " entreprises employant des salariés » dépendent moins du champ couvert par les registres d'entreprises que ceux couvrant toutes les entreprises. Dans de nombreux pays, les principales sources de données utilisées par ces registres sont les registres administratifs fiscaux et de lemploi, qui amènent souvent à ne prendre en compte que les entreprises affichant un certain seuil de chiffre diaffaires ou dיeffectif. En outre, les seuils, notamment les seuils de nature monétaire, peuvent évoluer dans le temps en fonction par exemple de l'inflation et de la politique budgétaire, lesquelles sont susceptibles d'altérer les comparaisons chronologiques et internationales des taux de décès. L'utilisation du seuil d'un salarié améliore la comparabilité car elle permet d'exclure les très petites unités, qui sont les plus sujettes aux variations de seuil.

La collecte des données concernant les décès d'entreprises se fait avec un décalage chronologique plus important que pour les naissances d'entreprises. Il faut en effet confirmer l'événement en vérifiant que l'entreprise n'a pas été réactivée (ou n'a pas eu de salariés) au cours des deux années suivant son décès.

Les données pour le Canada, les États-Unis et Israël font référence au nombre d'employés. Dans le graphique 4.9, les données font référence uniquement à la population des entreprises employant des salariés pour le Brésil, le Canada, les États-Unis, Israël et la Nouvelle-Zélande.

Pour l'Australie, la Corée et le Mexique, les décès d'entreprises et les indicateurs connexes ne tiennent pas compte des entreprises employant au moins un salarié qui sont devenues des entreprises sans salarié. Autrement dit, lorsqu'une entreprise employant des salariés devient une entreprise sans salarié, ce changement de statut n'est pas considéré comme un "décès d'entreprise employant des salariés ».

\section{Source}

OCDE, Statistiques structurelles et démographiques des entreprises (SDBS) (base de données), http://dx.doi.org/10.1787/sdbsdata-fr.

Counts of Australian Businesses, including Entries and Exits. 8165.0, http://dx.doi.org/10.1787/sdbs-data-fr.

\section{Pour en savoir plus}

Ahmad, N. (2006), " A Proposed Framework for Business Demography Statistics ", OECD Statistics Working Papers, vol. 2006, n³, Éditions OCDE, Paris, http://dx.doi. org/10.1787/145777872685.

OCDE (2010), Structural and Demographic Business Statistics, Éditions OCDE, Paris, http://dx.doi.org/10.1787/9789264072886-en.

OCDE/Eurostat (2008), Eurostat-OECD Manual on Business Demography Statistics, Éditions OCDE, Paris, http://dx.doi. org/10.1787/9789264041882-en. 
Graphique 4.9. Décès des entreprises sans salarié et des entreprises employant des salariés et destructions d'emplois, secteur marchand

En pourcentage, 2014 ou dernière année disponible

$\square$ Part des destructions d'emplois dues aux décès d'entreprises employant des salariés dans l'emploi total - Part des décès d'entreprises employant des salariés dans le nombre total d'entreprises

- Part des décès d'entreprises sans salarié dans le nombre total d'entreprises

- Part des destructions d'emplois dues aux décès d'entreprises sans salarié dans l'emploi total

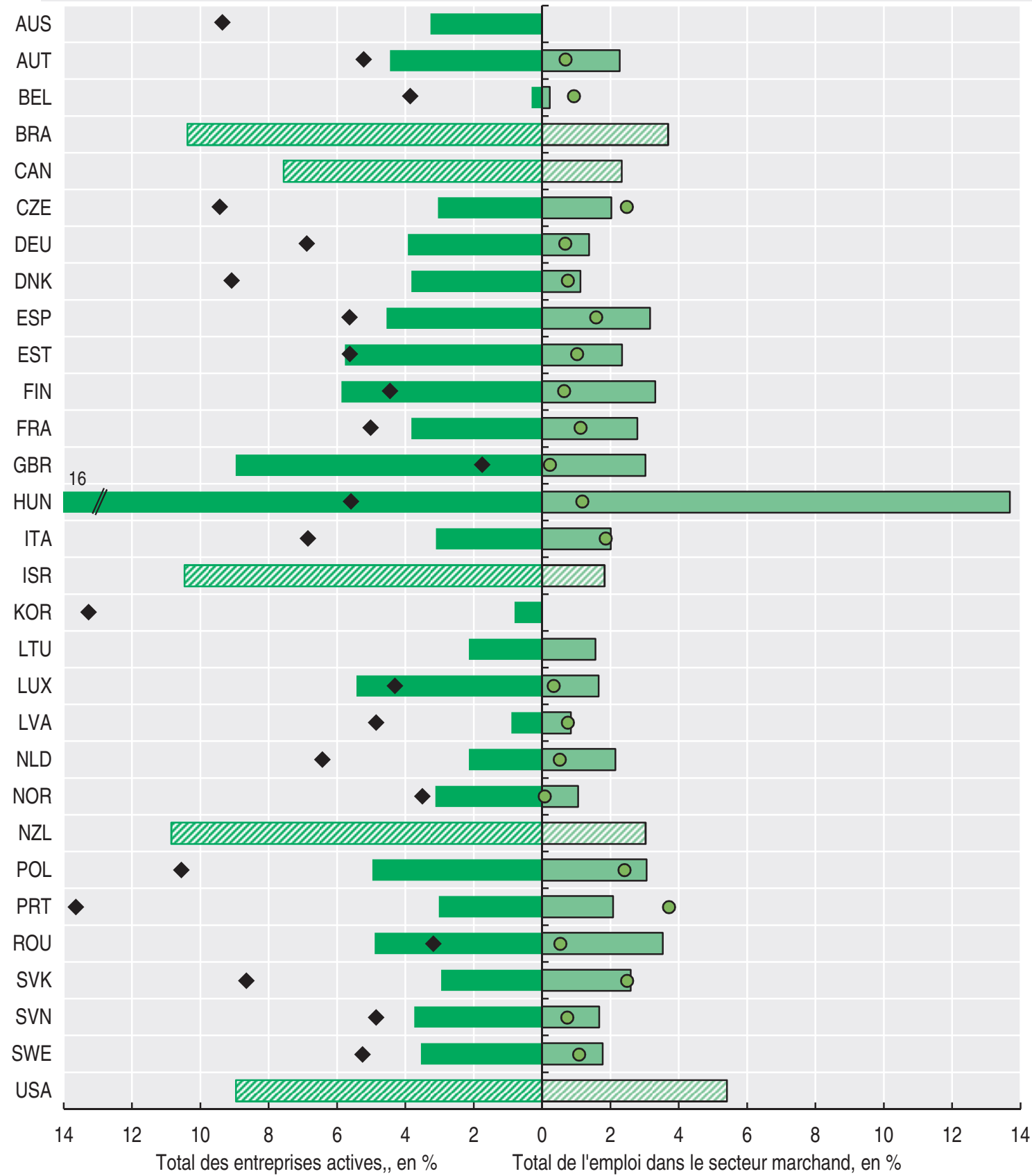




\section{DYNAMISME DES ENTREPRISES ET CRÉATION D'EMPLOIS}

\section{Décès d'entreprises}

Graphique 4.10. Nombre de décès d'entreprises employant des salariés et sans salarié, secteur marchand En milliers d'entreprises, 2014 ou dernière année disponible

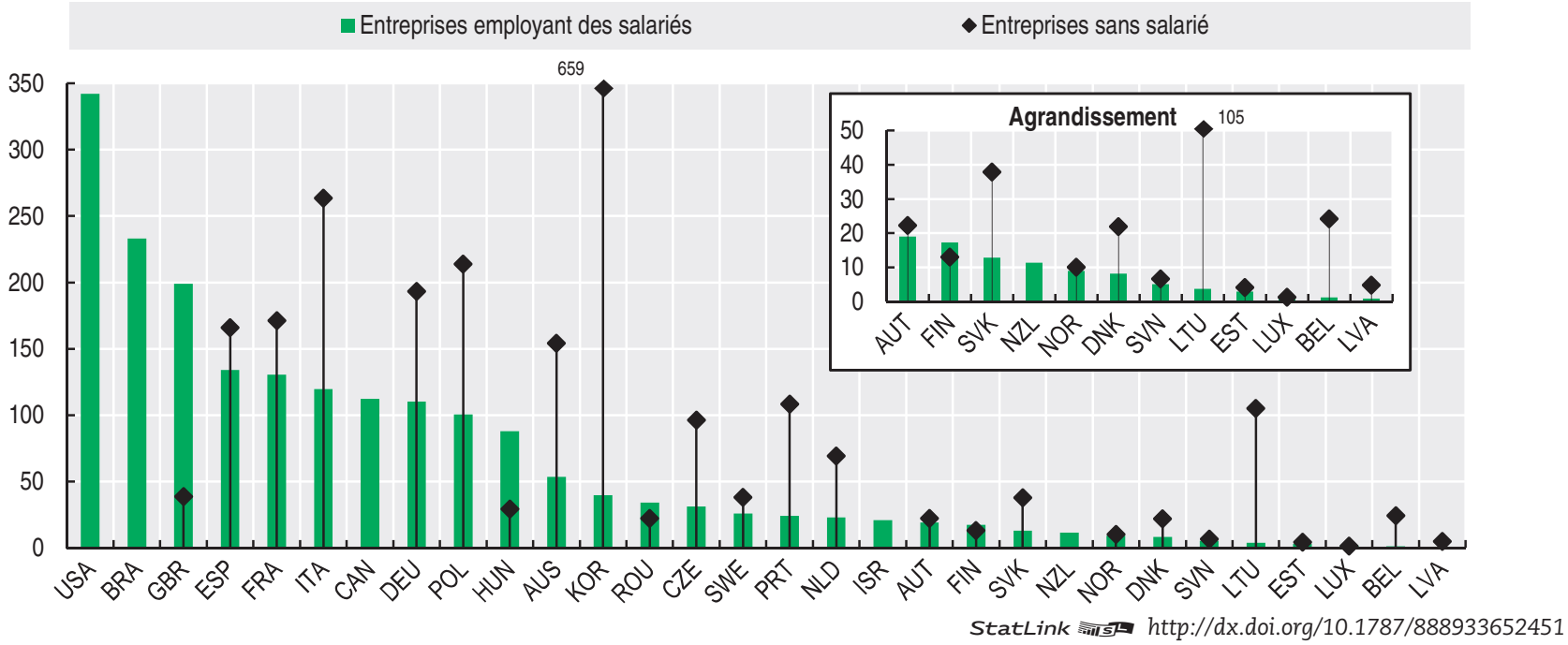

Graphique 4.11. Taux de décès des entreprises employant des salariés, secteur marchand

Nombre des décès d'entreprises en pourcentage des entreprises actives employant des salariés, 2014 ou dernière année disponible

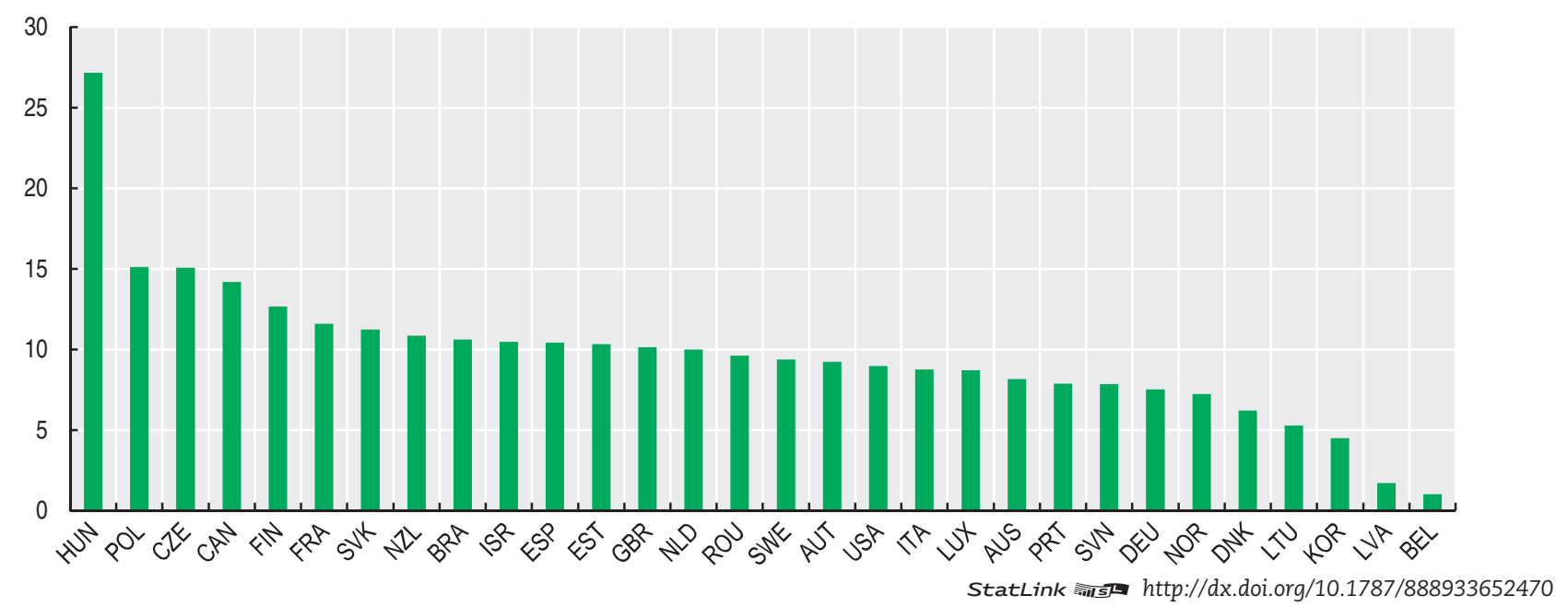


Graphique 4.12. Taux de décès des entreprises employant des salariés, principaux secteurs En pourcentage des entreprises actives employant des salariés, 2014 ou dernière année disponible

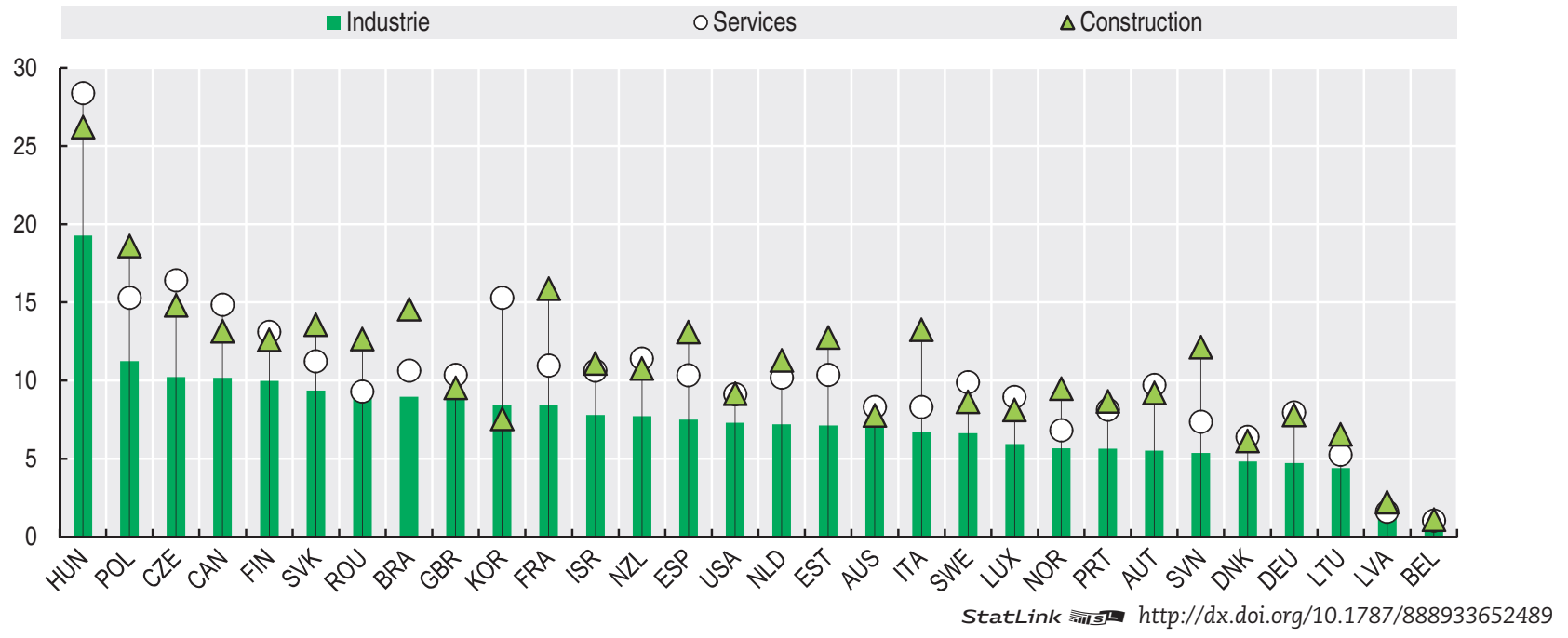

Graphique 4.13. Destructions d'emplois dues aux décès d'entreprises employant des salariés, secteur marchand Nombre de personnes employées, en milliers, 2014 ou dernière année disponible

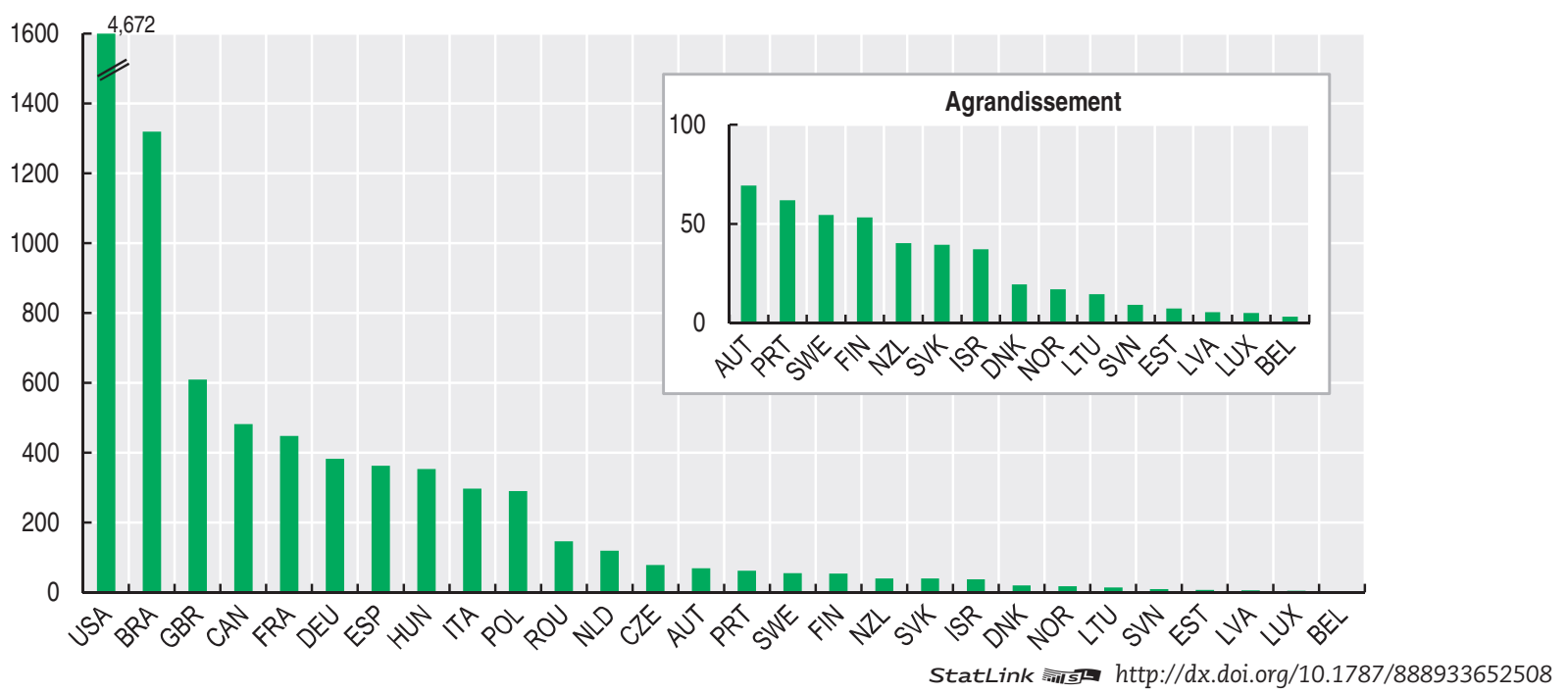




\section{DYNAMISME DES ENTREPRISES ET CRÉATION D'EMPLOIS}

\section{Décès d'entreprises}

Graphique 4.14. Destructions d'emplois dues aux décès d'entreprises employant des salariés, principaux secteurs Pourcentage des destructions d'emplois totales dans le secteur marchand, 2014 ou dernière année disponible

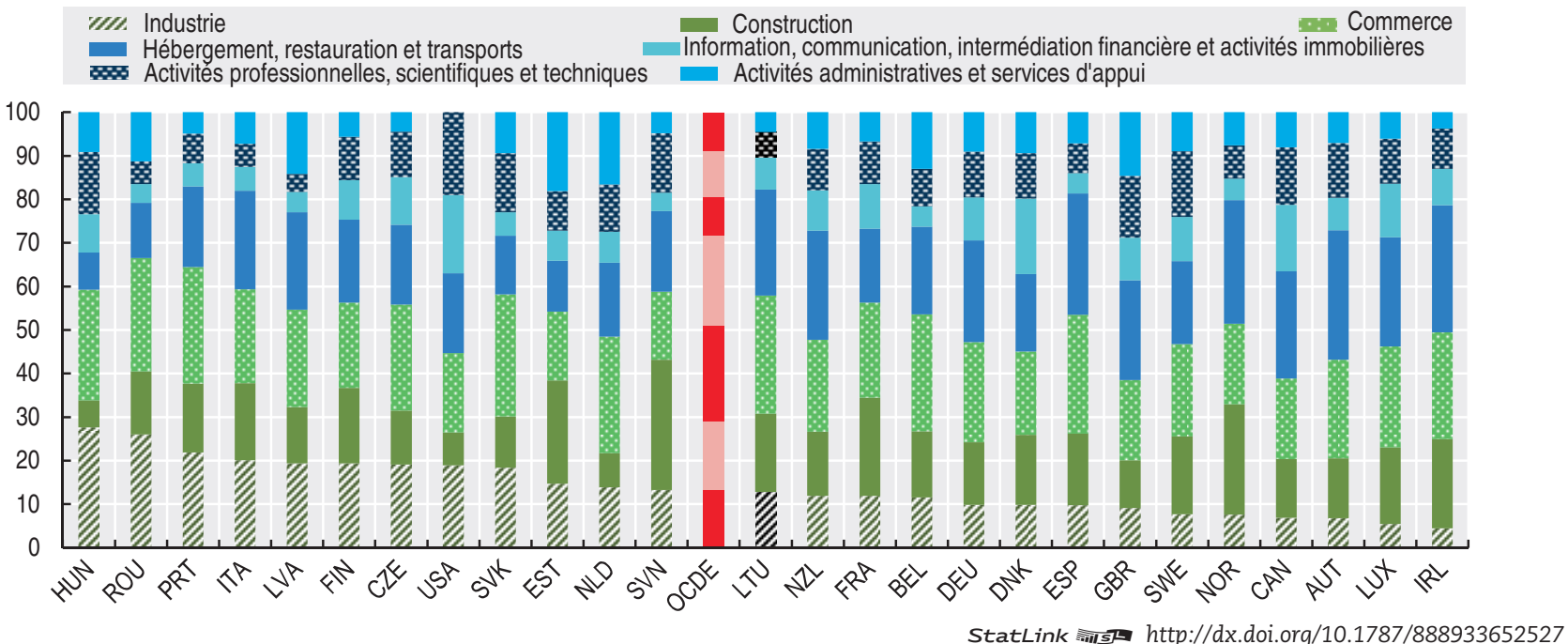

Graphique 4.15. Taux de destruction d'emplois par activité économique

Emplois concernés par les décès d'entreprises employant des salariés en pourcentage de l'emploi total, secteur marchand, 2014 ou dernière année disponible

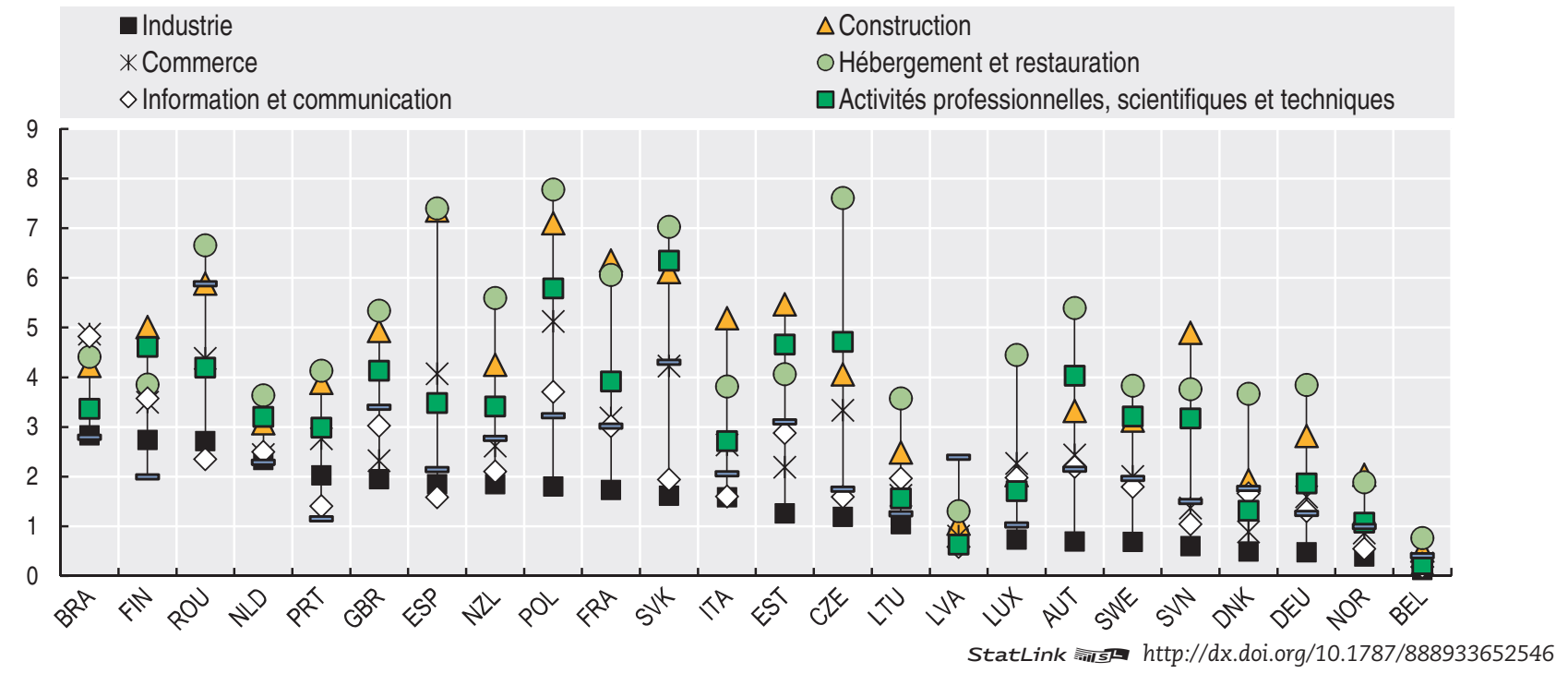


Graphique 4.16. Décès des entreprises employant des salariés, par taille et par secteur

Pourcentage (proportion des classes de taille) et nombre de personnes employées par l'entreprise au moment du décès (effectif moyen), 2014 ou dernière année disponible

Industrie

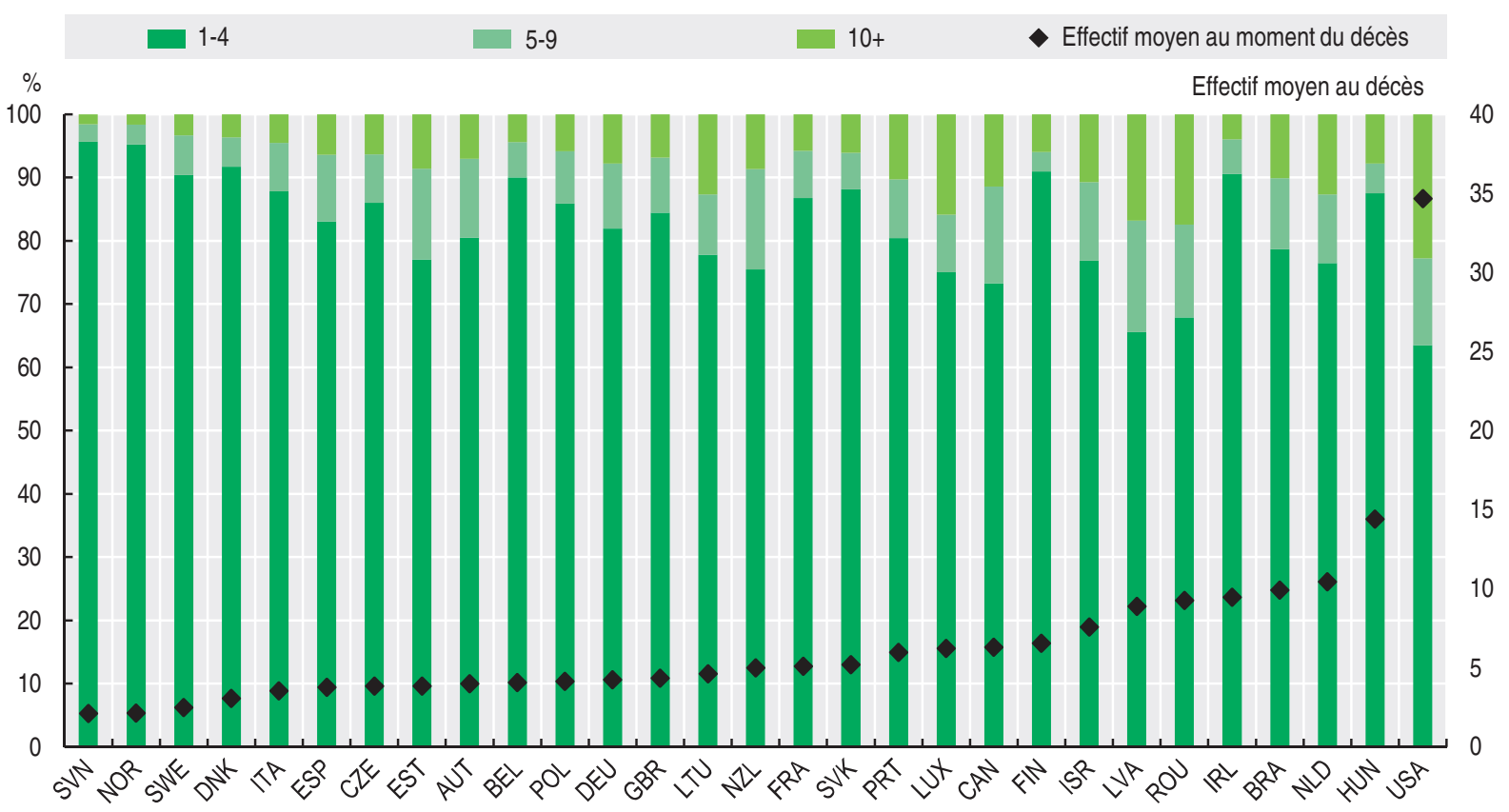

\section{Services}

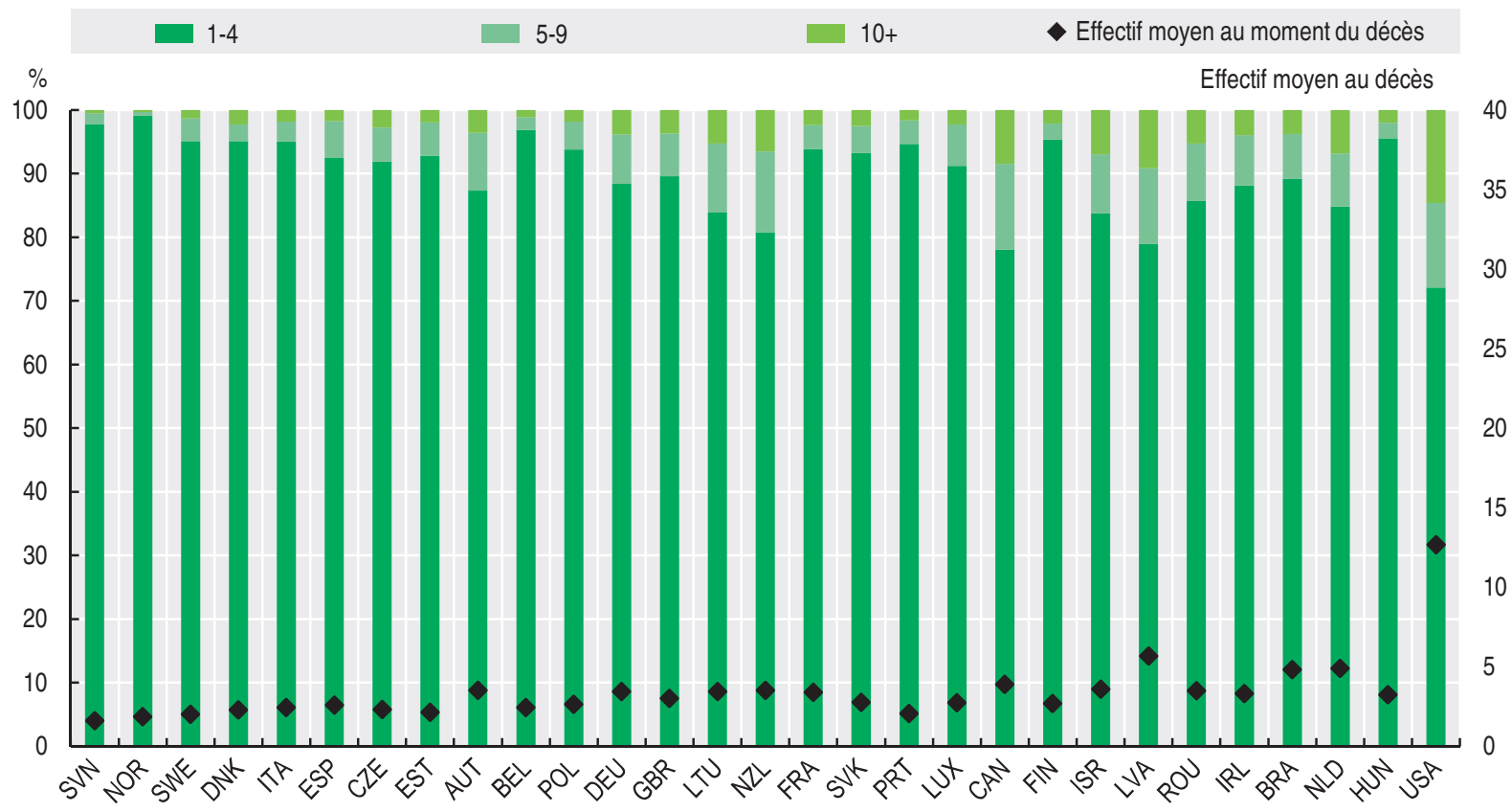

StatLink तiाst $h$ ttp://dx.doi.org/10.1787/888933652565 


\section{À savoir}

- En 2014, le taux de renouvellement des entreprises employant des salariés s'élevait environ à $20 \%$ en moyenne dans la zone OCDE ; seul un petit nombre de pays affichait un taux beaucoup plus faible (Belgique) ou beaucoup plus élevé (Hongrie).

- Dans plusieurs pays, tels que l'Autriche, le Canada, la Hongrie, le Luxembourg, la Nouvelle-Zélande, la République tchèque et la Slovénie, le taux de renouvellement a augmenté en 2014 par rapport au niveau qu'il affichait au début de la crise mondiale.

- La création nette d'emplois associée au renouvellement des entreprises employant des salariés varie fortement entre les pays. Ainsi, en Lettonie et en Slovénie, la création d'emplois due au renouvellement représentait plus de $55 \%$ du changement total de l'emploi dans le secteur marchand entre 2013 et 2014, et environ 25 \% en Norvège, au RoyaumeUni et au Brésil. Dans plusieurs autres pays, le renouvellement des entreprises s'est traduit à l'inverse par une destruction nette d'emplois, et représentait aux Pays-Bas et en Hongrie plus de $40 \%$ de la variation totale de l'emploi dans le secteur marchand entre 2013 et 2014.

\section{Pertinence}

Le taux de renouvellement, c'est-à-dire la somme des taux de naissances et de décès d'entreprises, fournit une mesure de la fréquence à laquelle des entreprises nouvelles se créent et des entreprises en activité ferment. Dans la plupart des économies, le nombre de naissances et de décès d'entreprises représente une part considérable du nombre total d'entreprises. L'indicateur rend compte du niveau de " destruction créatrice " d'un pays et corrobore, par exemple, l'analyse de la contribution du dynamisme du secteur des entreprises à la hausse globale de la productivité.

\section{Définitions}

Le taux de renouvellement des entreprises employant des salariés est calculé en additionnant le taux de naissance et le taux de décès de ces entreprises. Les données sur les naissances et les décès d'entreprises employant des salariés qui sont utilisées pour obtenir le taux de renouvellement de cette catégorie d'entreprise sont conformes aux définitions recommandées dans le manuel intitulé Eurostat-OECD Manual on Business Demography Statistics (2008).

Le taux de renouvellement des entreprises employant des salariés n'inclut pas les entrées et les sorties dues à des fusions, dissolutions, scissions, reprises ou restructurations au sein d'un groupe d'entreprises. Il exclut également les entrées et les sorties qui, au sein d'une sous-population, ne résultent que d'un changement d'activité.

La création nette d'emplois due aux naissances et décès d'entreprises employant des salariés correspond à la différence entre le nombre de personnes occupées dans les entreprises créées au cours de la période de référence $t$, et le nombre de personnes occupées dans les entreprises détruites en $t$.

Informations sur les données concernant Israël : http:// dx.doi.org/10.1787/888932315602.

\section{Comparabilité}

Comme précisé dans les sections précédentes, les indicateurs concernant les "entreprises employant des salariés " permettent une meilleure comparaison internationale que ceux englobant toutes les entreprises, dans la mesure où ces derniers dépendent du champ couvert et des seuils utilisés par les registres dientreprises.

Pour l'Australie, les naissances et décès d'entreprises et les indicateurs connexes ne tiennent pas compte des entreprises sans salarié devenues des entreprises employant au moins un salarié, et vice versa. Autrement dit, lorsqu'une entreprise sans salarié devient une entreprise employant des salariés, ce changement de statut n'est pas considéré comme une « naissance d'entreprise employant des salariés » et lorsqu’une entreprise employant des salariés devient une entreprise sans salarié, ce changement de statut n'est pas considéré comme un « décès d'entreprise employant des salariés ".

\section{Source}

OCDE, Statistiques structurelles et démographiques des entreprises (SDBS) (base de données), http://dx.doi.org/10.1787/sdbsdata-fr.

\section{Pour en savoir plus}

Ahmad, N. (2006), " A Proposed Framework for Business Demography Statistics ", OECD Statistics Working Papers, vol. 2006, $\mathrm{n}^{\circ} 3$, Éditions OCDE, Paris, http://dx.doi. org/10.1787/145777872685.

OCDE/Eurostat (2008), Eurostat-OECD Manual on Business Demography Statistics, Éditions OCDE, Paris, http://dx.doi. org/10.1787/9789264041882-en. 
Graphique 4.17. Taux de renouvellement des entreprises employant des salariés, secteur marchand En pourcentage du total des entreprises employant des salariés, 2014 ou dernière année disponible

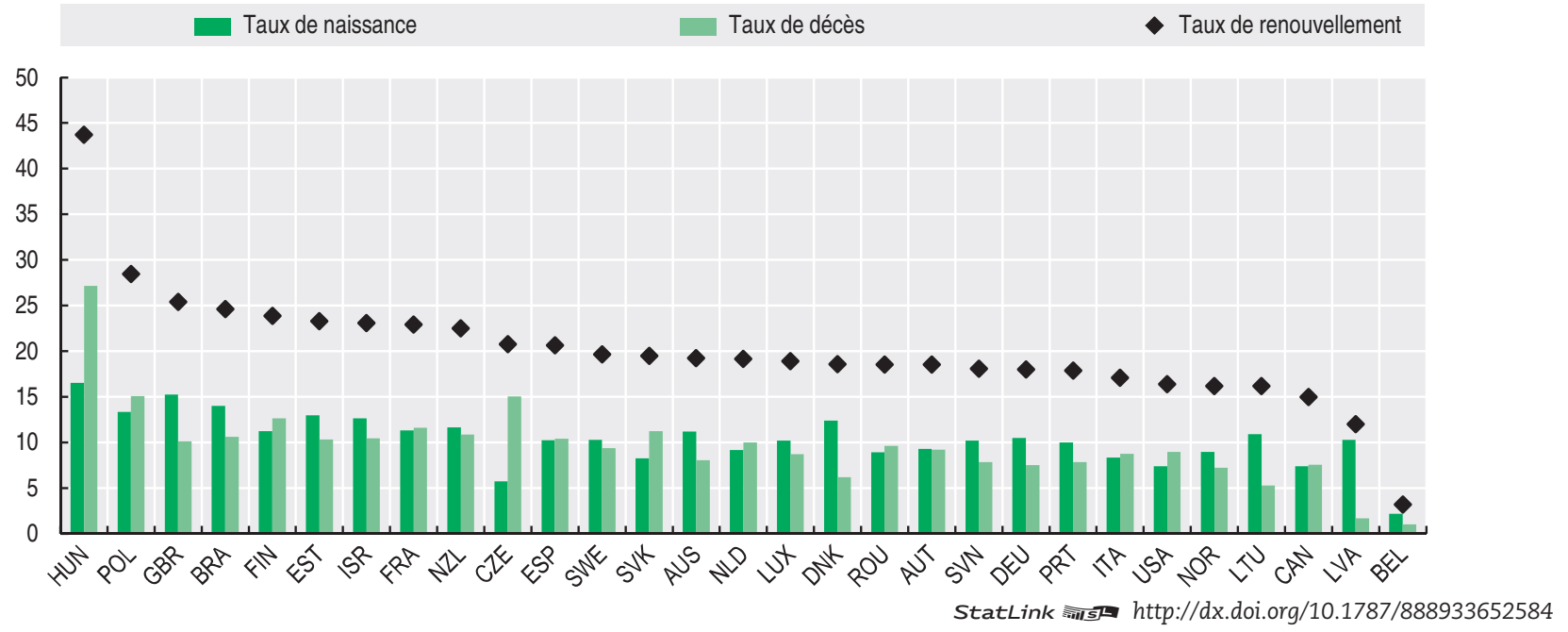

Graphique 4.18. Évolution du taux de renouvellement des entreprises employant des salariés, secteur marchand En pourcentage du nombre total des entreprises employant des salariés

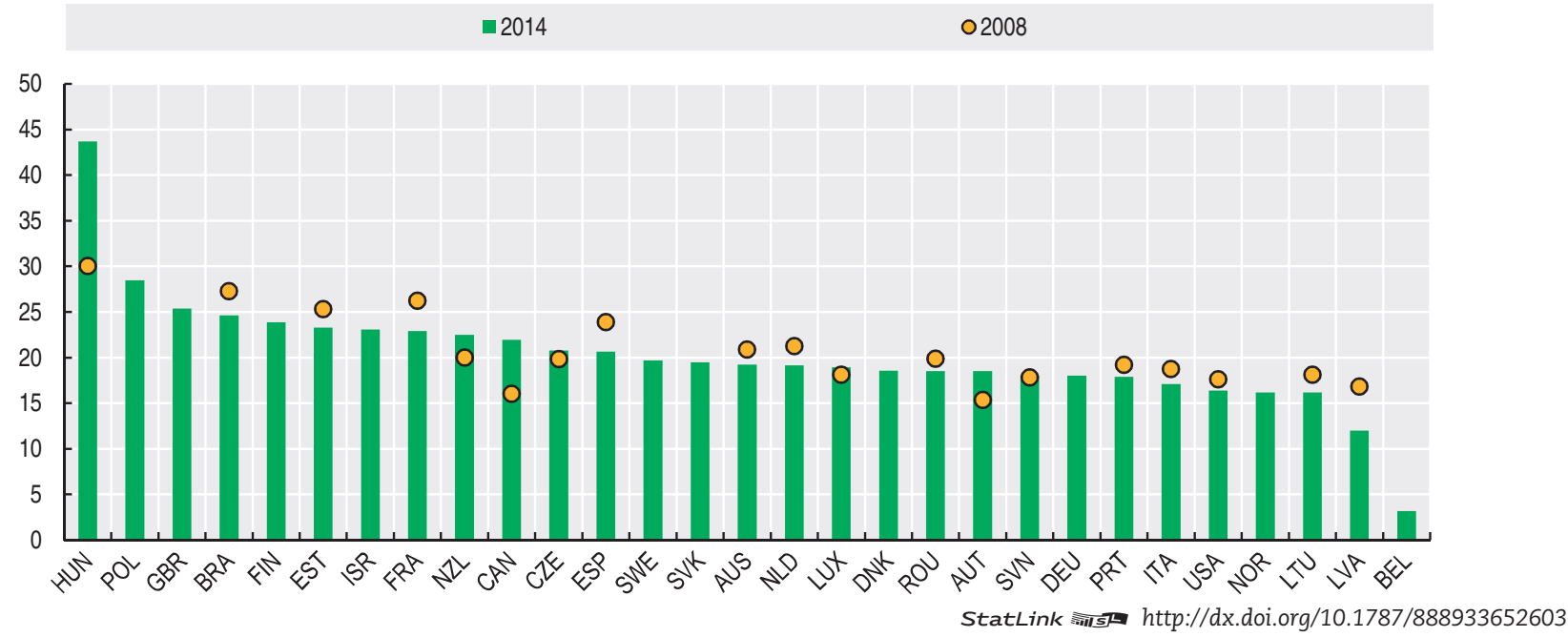

Graphique 4.19. Taux de renouvellement des entreprises et créations nettes d'emplois, secteur marchand Contributions et pourcentage de variation entre 2013 et 2014, ou dernière année disponible

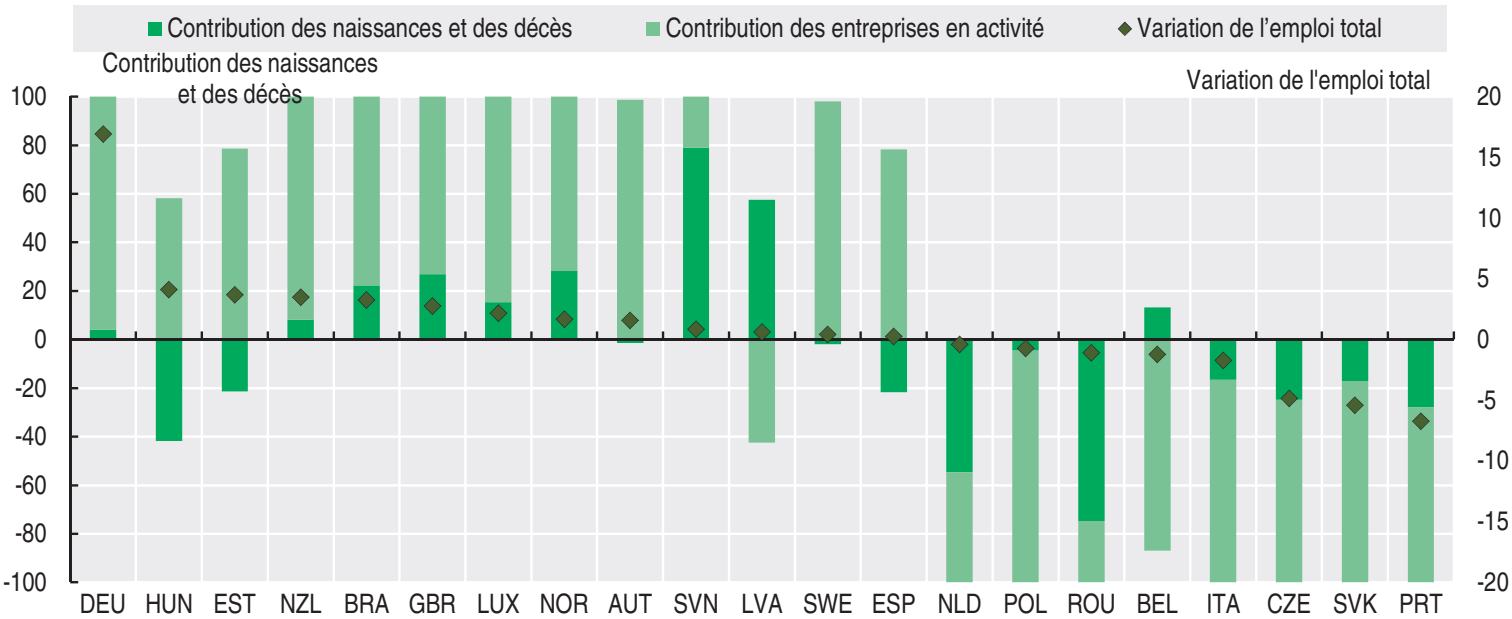




\section{À savoir}

- De nombreuses entreprises de création récente ne survivent pas au-delà de leurs premières années d'activité, même s'il existe des écarts importants dans ce domaine entre les différents pays. Le taux de survie à un an des entreprises employant des salariés créées en 2013 était supérieur à $90 \%$ en Suède, aux États-Unis, au Luxembourg, en Lituanie et au Royaume-Uni, mais s'établissait entre $60 \%$ et $70 \%$ en République tchèque et en Pologne, et au-dessous de $55 \%$ en République slovaque.

- Les taux de survie sont en général plus élevés dans l'industrie que dans les services ou la construction, et pour les entreprises créées avec cinq salariés ou plus.

- En 2014, les jeunes entreprises représentaient environ $20 \%$ ou plus de la totalité des entreprises employant des salariés dans tous les pays à l'exception de la Belgique, et plus de $30 \%$ au Royaume-Uni, en Hongrie, au Brésil, en Israël et en Pologne.

- En 2014, les jeunes entreprises représentaient moins de $10 \%$ de l'emploi du secteur marchand dans la plupart des pays.

\section{Pertinence}

L'étude des performances des entreprises après leur naissance est tout aussi importante que l'analyse de leur taux de naissance. Des taux d'échec très élevés peuvent décourager les aspirants entrepreneurs aussi bien que les créanciers potentiels, ce qui pourrait entraver l'innovation et la croissance à long terme. L'étude de la part de l'emploi imputable aux jeunes entreprises qui survivent permet de mieux comprendre le rôle que jouent les différentes entreprises dans l'évolution globale de l'emploi au sein de l'économie.

\section{Définitions}

Le nombre d'entreprises qui, l'année t, ont survécu $n$ années, correspond au nombre d'entreprises qui ont eu au moins un salarié pour la première fois l'année $t-n$ et sont toujours en activité l'année t. Cette définition de la survie exclut les cas dans lesquels les entreprises fusionnent ou sont absorbées par une entreprise qui existait l'année $\mathrm{t}-\mathrm{n}$.

Le taux de survie des entreprises employant des salariés dans un secteur (classe de taille) x mesure le nombre d'entreprises d'une cohorte de naissances spécifique dans le secteur (classe de taille) x qui ont passé différentes échéances annuelles. Le taux de survie sur $\mathrm{n}$ années des entreprises employant des salariés pour une année de référence t est exprimé par le nombre d'entreprises ayant survécu $\mathrm{n}$ années en pourcentage du nombre total d'entreprises ayant déclaré au moins un salarié pour la première fois l'année $t-n$.

La proportion d'entreprises de $n$ années employant des salariés pour une année $t$ désigne le nombre d'entreprises ayant survécu $\mathrm{n}$ années, exprimé en pourcentage de la population totale des entreprises employant des salariés en $t$.

Comme le définit la présente publication, les jeunes entreprises employant des salariés englobent toutes les entreprises employant des salariés qui ont jusqu'à deux ans d'âge, c'est-à-dire celles qui sont de création récente, plus celles âgées d'un an ou de deux ans.
La part de l'emploi imputable aux jeunes entreprises employant des salariés correspond au nombre de personnes occupées au sein d'entreprises employant des salariés âgées de deux ans au plus, divisé par le nombre total de personnes occupées dans les entreprises employant des salariés.

La taille moyenne des jeunes entreprises employant des salariés est exprimée par le nombre de personnes occupées au cours de la période de référence $(\mathrm{t})$ parmi les jeunes entreprises employant des salariés et créées durant la période $t$, divisé par le nombre de jeunes entreprises employant des salariés et créées en $t$.

Informations sur les données concernant Israël : http:// dx.doi.org/10.1787/888932315602

\section{Comparabilité}

Les données présentées se rapportent à l'ensemble de la population des entreprises employant des salariés. Pour le Canada, les États-Unis et Israël, les statistiques sur l'emploi dans les entreprises qui se créent et qui survivent se réfèrent non pas au nombre de personnes occupées mais au nombre de salariés.

Les statistiques qui sont publiées ici au sujet de la survie des entreprises employant des salariés sont conformes à la définition figurant dans le manuel Eurostat-OECD Manual on Business Demography Statistics (2008).

Pour l'Australie, la Corée et le Mexique, les naissances d'entreprises et les indicateurs connexes ne tiennent pas compte des entreprises sans salarié devenues des entreprises employant au moins un salarié, et vice versa. Autrement dit, lorsqu'une entreprise sans salarié devient une entreprise employant des salariés, ce changement de statut n'est pas considéré comme une " naissance d'entreprise employant des salariés ".

\section{Source}

OCDE, Statistiques structurelles et démographiques des entreprises (SDBS) (base de données). http://dx.doi.org/10.1787/sdbs-data-fr.

\section{Pour en savoir plus}

Ahmad, N. (2006), " A Proposed Framework for Business Demography Statistics ", OECD Statistics Working Papers, vol. 2006, n³, Éditions OCDE, Paris, http://dx.doi. org/10.1787/145777872685.

Decker, R. A., J. Haltiwanger, R.S. Jarmin et J. Miranda (2016), "Where has all the skewness gone? The decline in highgrowth (young) firms in the U.S. ", European Economic Review, http://www.nber.org/papers/w21776.

OCDE/Eurostat (2008), Eurostat-OECD Manual on Business Demography Statistics, Éditions OCDE, Paris, http://dx.doi. org/10.1787/9789264041882-en. 
Graphique 4.20. Taux de survie des entreprises d'un an employant des salariés En pourcentage, cohorte de 2013

Par secteur

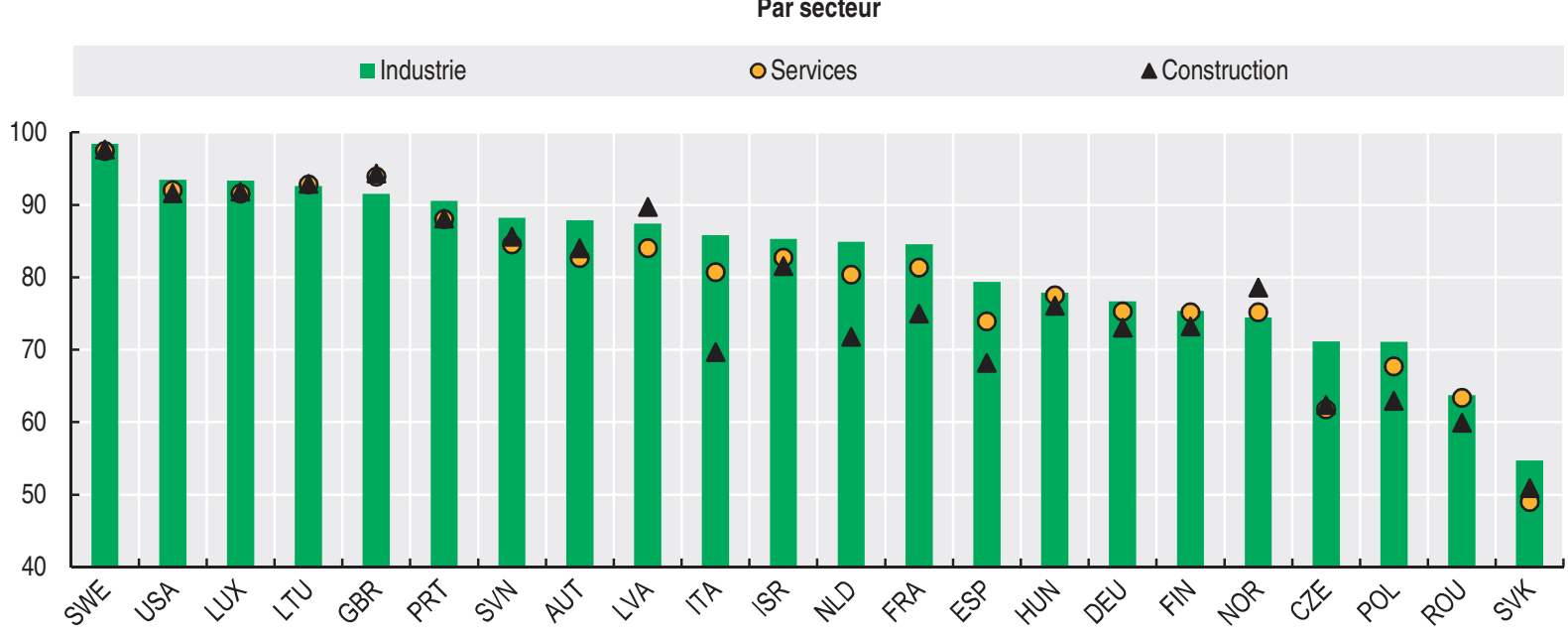

Par taille

$\square 1-4 \quad \square 5-9 \quad \square 10+$

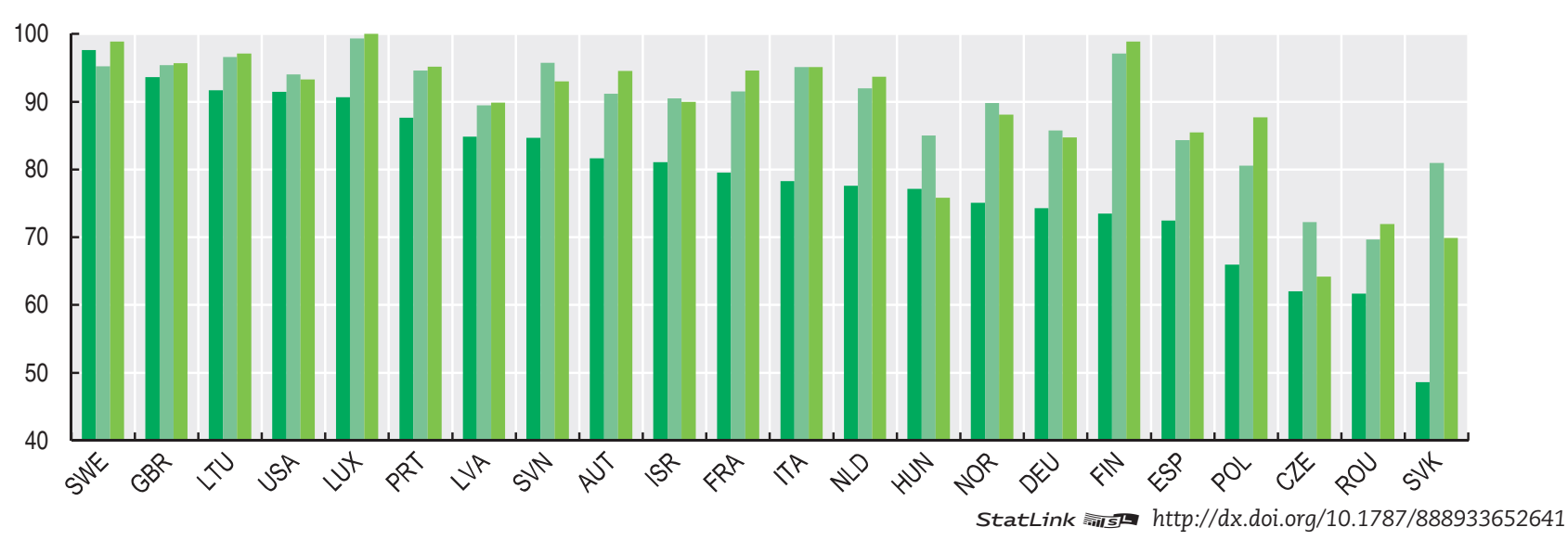

Graphique 4.21. Proportion des jeunes entreprises et de leur effectif, secteur marchand

En pourcentage de toutes les entreprises employant des salariés et de l'effectif dans la totalité des entreprises employant des salariés, 2014 ou dernière année disponible

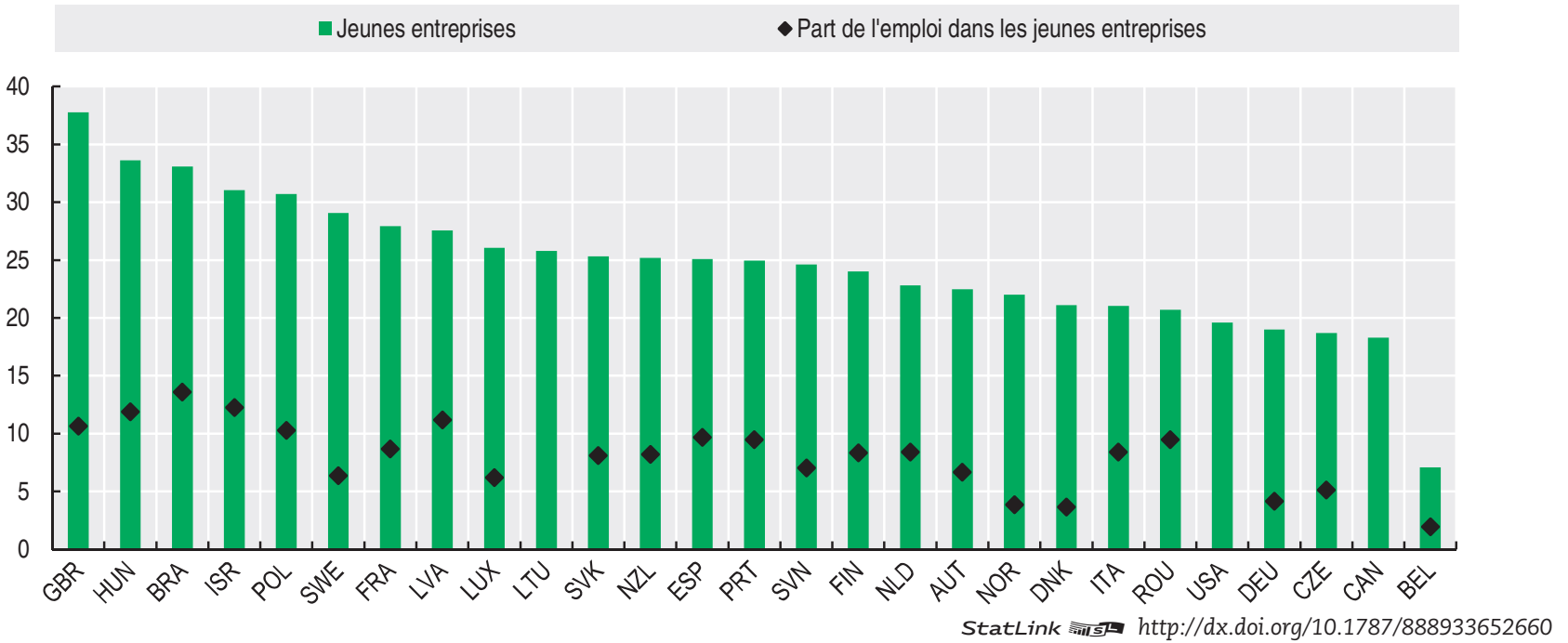




\section{DYNAMISME DES ENTREPRISES ET CRÉATION D'EMPLOIS}

\section{Jeunes entreprises}

Graphique 4.22. Taille moyenne des jeunes entreprises employant des salariés

Effectif des entreprises âgées de 0 à 2 ans divisé par leur nombre, 2014 ou dernière année disponible

\section{Industrie}
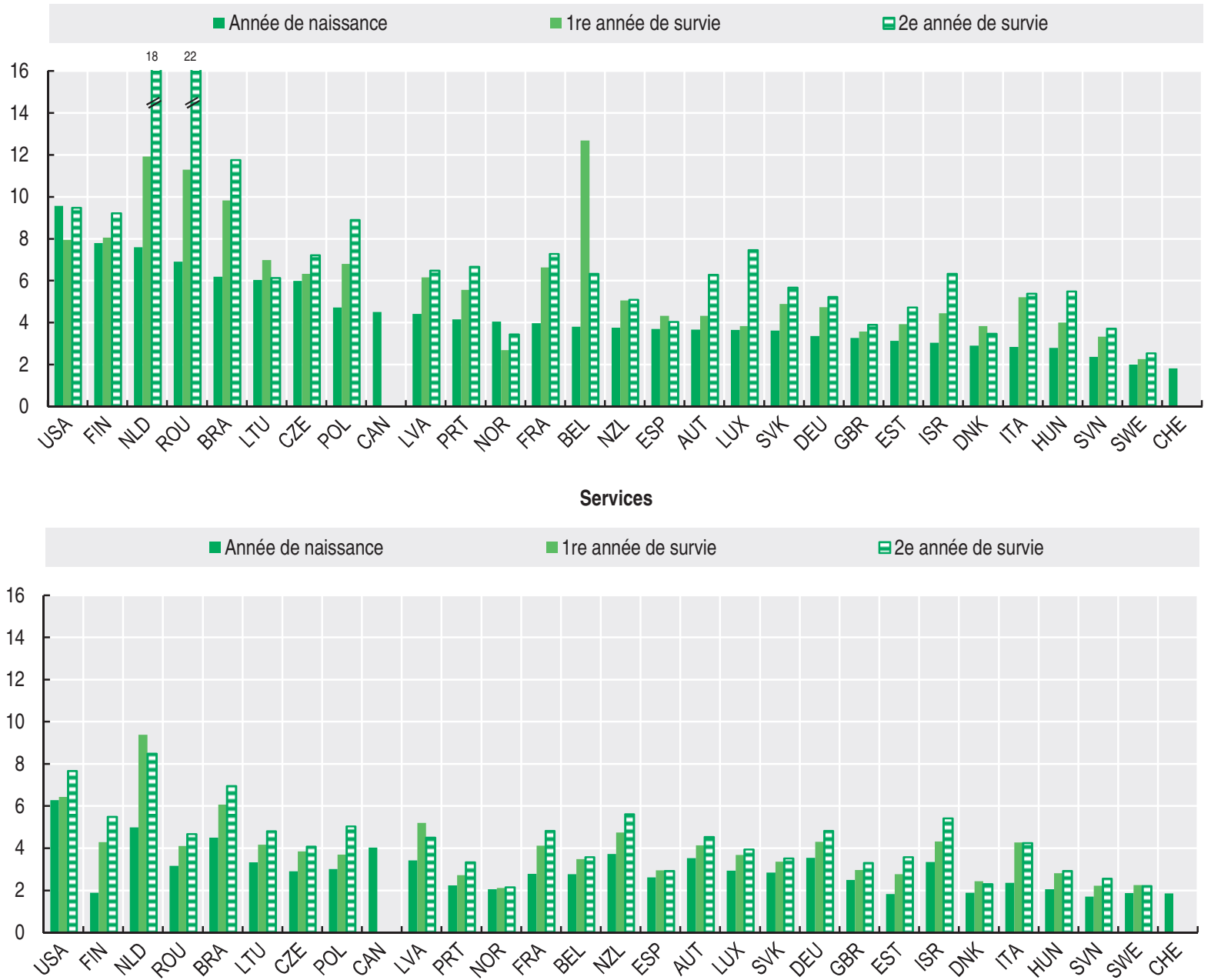

Construction

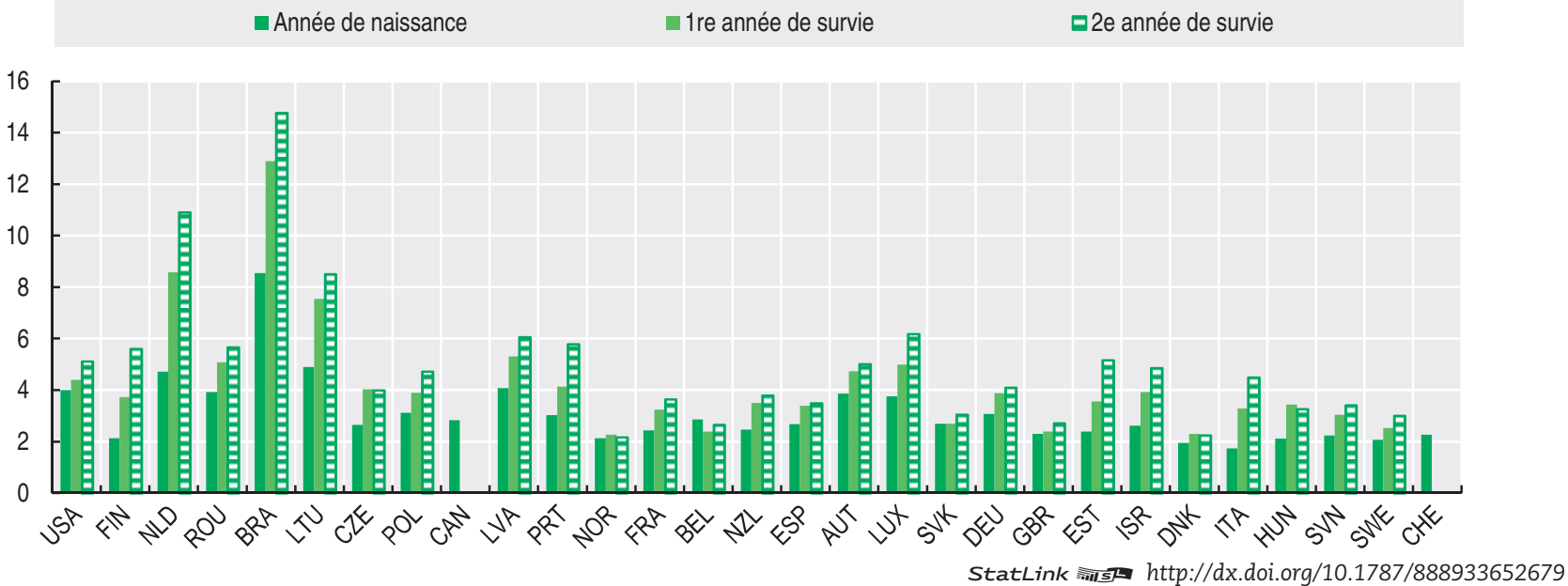


Graphique 4.23. Proportion des jeunes entreprises employant des salariés dans la population des entreprises En pourcentage du total des entreprises employant des salariés, 2014 ou dernière année disponible

Industrie

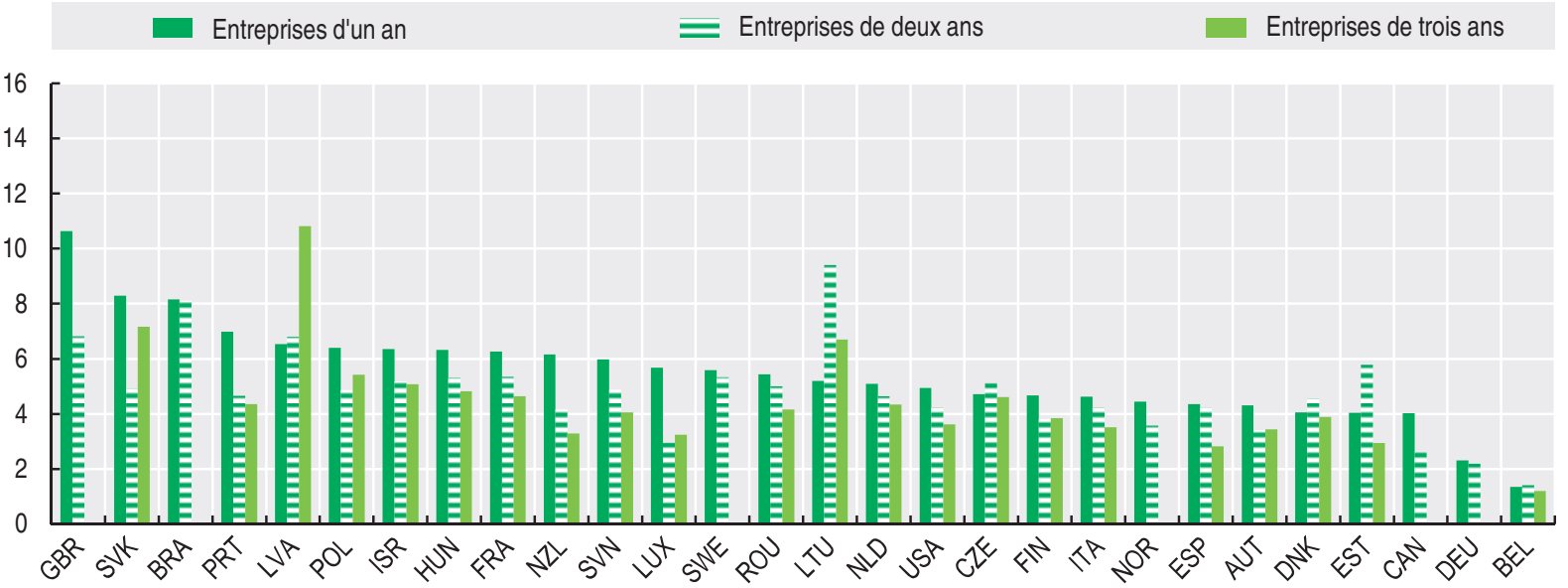

Services
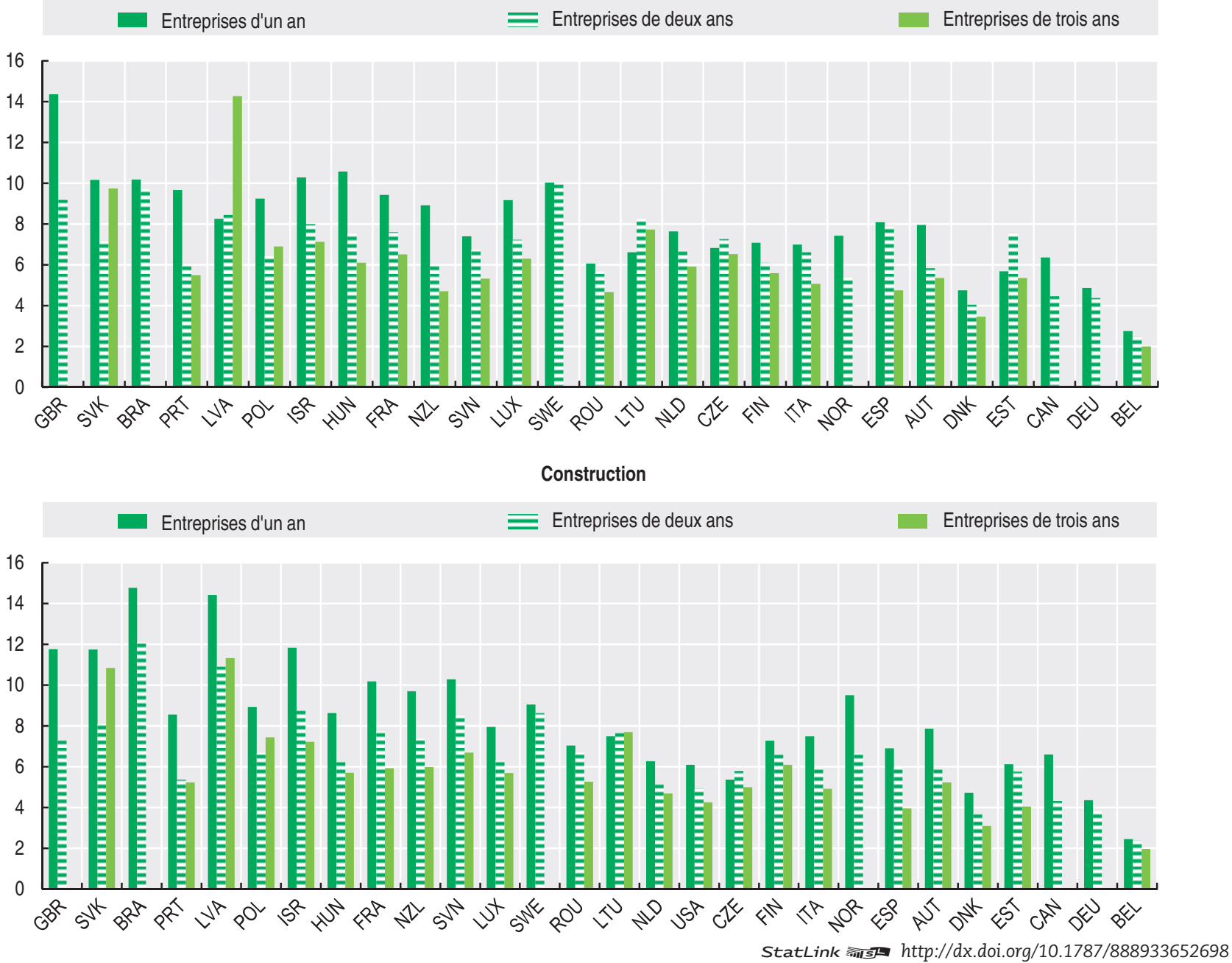


\section{À savoir}

- Bien que d'un nombre relativement restreint, les entreprises à forte croissance contribuent de manière disproportionnée à la création d'emplois. Ainsi, en 2014, elles représentaient environ $20 \%$ de l'emploi dans la totalité des entreprises employant 10 salariés au minimum en Irlande, en Israël ou au Royaume-Uni.

- Le taux d'entreprises à forte croissance varie fortement entre les pays ainsi que d'un secteur à l'autre, ce qui rend compte de la spécialisation relative ou des avantages comparatifs des pays dans certaines activités. Ainsi, en France et en Suède, le taux d'entreprises à forte croissance est plus élevé dans les services que dans l'industrie, alors que la Hongrie et la Lettonie présentent une situation opposée.

- Cependant, au sein des pays, les tendances relatives à l'évolution des taux d'entreprises à forte croissance sont généralement homogènes, ce qui tendrait à démontrer que les moteurs d'une forte croissance sont fortement influencés par l'environnement des affaires global. Les pays où la proportion d'entreprises à forte croissance est relativement importante dans une activité spécifique affichent en général également une forte proportion d'entreprises à forte croissance dans d'autres activités.

- En 2014, ce sont les activités administratives et les services d'appui, notamment dans les services de sécurité et d'enquête, la programmation informatique et les activités liées à l'emploi, qui affichaient la proportion la plus élevée de " gazelles ", c'est-à-dire de jeunes entreprises à forte croissance.

\section{Pertinence}

Les entreprises à forte croissance sont d'importantes sources de création d'emplois et de richesses. Un petit groupe d'entreprises à forte croissance est à l'origine d'un nombre extrêmement élevé de créations d'emplois.

\section{Définitions}

Les entreprises à forte croissance sont des entreprises qui affichent une croissance annuelle moyenne du nombre de salariés de plus de $20 \%$ sur une période de trois ans, et qui comptent au moins 10 salariés au début de la période d'observation (Eurostat-OECD Manual on Business Demography Statistics, 2008).

Dans l'Union européenne, le Règlement d'exécution (UE) $n^{\circ} 439 / 2014$ définit comme suit les entreprises à forte croissance : entreprises marchandes employant au moins 10 salariés au début de leur croissance, et dont la croissance moyenne annuelle du nombre de salariés dépasse $10 \%$ par an pendant une période de trois ans.

La présente section utilise les deux définitions précitées des entreprises à forte croissance (respectivement une croissance supérieure à $20 \%$ et à $10 \%$ ).
La part des entreprises à forte croissance correspond au nombre d'entreprises à forte croissance exprimé en pourcentage de la population des entreprises d'au moins 10 salariés.

L'effectif moyen dans les entreprises à forte croissance s'obtient en divisant le nombre de salariés dans les entreprises à forte croissance pendant la période de référence par le nombre d'entreprises à forte croissance pendant cette même période.

Les " gazelles " sont un sous-groupe des entreprises à forte croissance. Il s'agit d'entreprises à forte croissance qui emploient des salariés depuis cinq ans tout au plus. La proportion des gazelles est le nombre de gazelles exprimé en pourcentage de la population des entreprises d'au moins 10 salariés.

Informations sur les données concernant Israël : http:// dx.doi.org/10.1787/888932315602

\section{Comparabilité}

Pour mesurer la population des entreprises à forte croissance, un seuil de dix salariés est fixé au début de chaque période d'observation pour éviter le biais lié aux petites entreprises. Le choix du seuil de la classe aura nécessairement un impact plus ou moins fort sur la représentativité des résultats selon la taille du pays.

S'agissant du Danemark, de l'Estonie, de la Finlande, de la France et du Portugal, une rupture de série est à noter en 2013 et pour la France et l'Irlande en 2014.

\section{Source}

OCDE, Statistiques structurelles et démographiques des entreprises (SDBS) (base de données), http://dx.doi.org/10.1787/sdbs-data-fr.

\section{Pour en savoir plus}

Ahmad, N. et D. Rude Petersen (2007), « High-Growth Enterprises and Gazelles ", Preliminary and Summary Sensitivity Analysis, Forums de l'OCDE, Paris, http://www.oecd.org/ document/31/0,3746,en_2825_499554_39151327_1_1_1_1,00. html.

Coad, A. et al. (2014), " High-growth firms: introduction to the special section ", Oxford Journals, Industrial and Corporate Change, http://icc.oxfordjournals.org/content/23/1/91.full.

OCDE/Eurostat (2008), Eurostat-OECD Manual on Business Demography Statistics, Éditions OCDE, Paris, http://dx.doi. org/10.1787/9789264041882-en. 
Graphique 4.24. Nombre d'entreprises à forte croissance et emploi, secteur marchand 2015 ou dernière année disponible

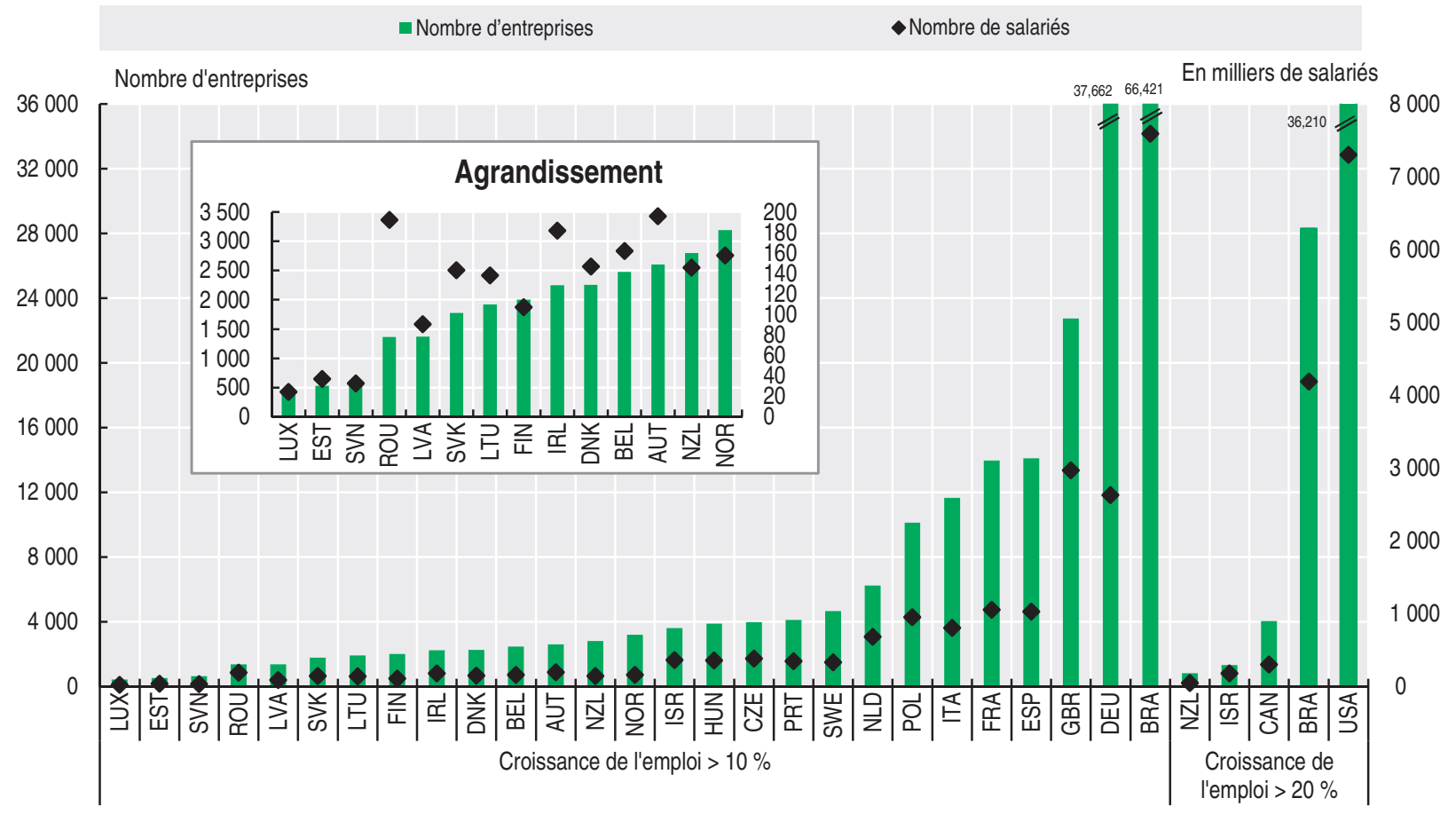

StatLink Ails http://dx.doi.org/10.1787/888933652717

Graphique 4.25. Effectif moyen dans les entreprises à forte croissance, principaux secteurs Nombre de salariés par entreprise et par secteur, 2014 ou dernière année disponible

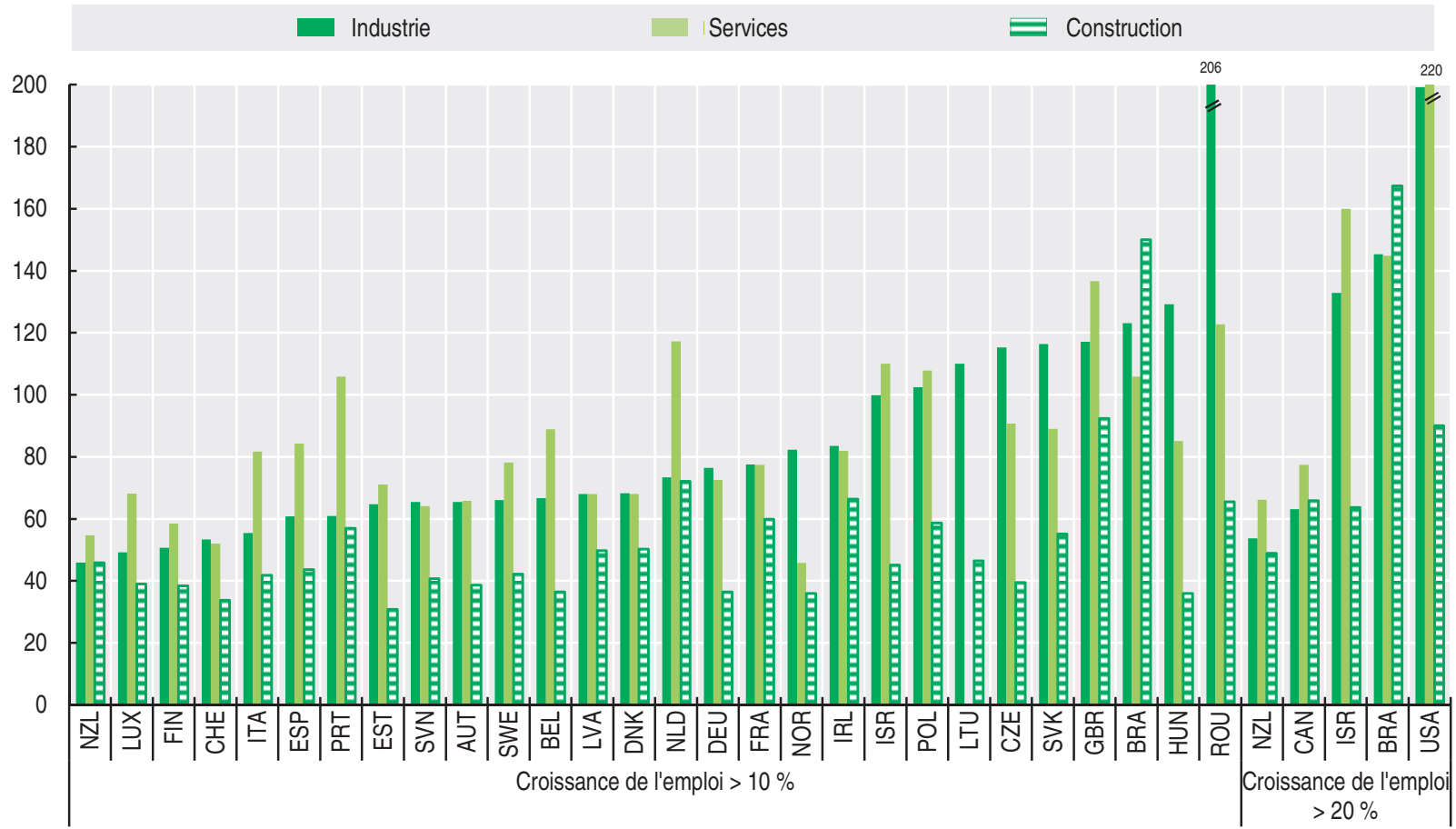




\section{DYNAMISME DES ENTREPRISES ET CRÉATION D'EMPLOIS}

\section{Taux d'entreprises à forte croissance}

Graphique 4.26. Part des entreprises à forte croissance (croissance de l'emploi supérieure à $10 \%$ ) En pourcentage des entreprises comptant dix salariés ou plus
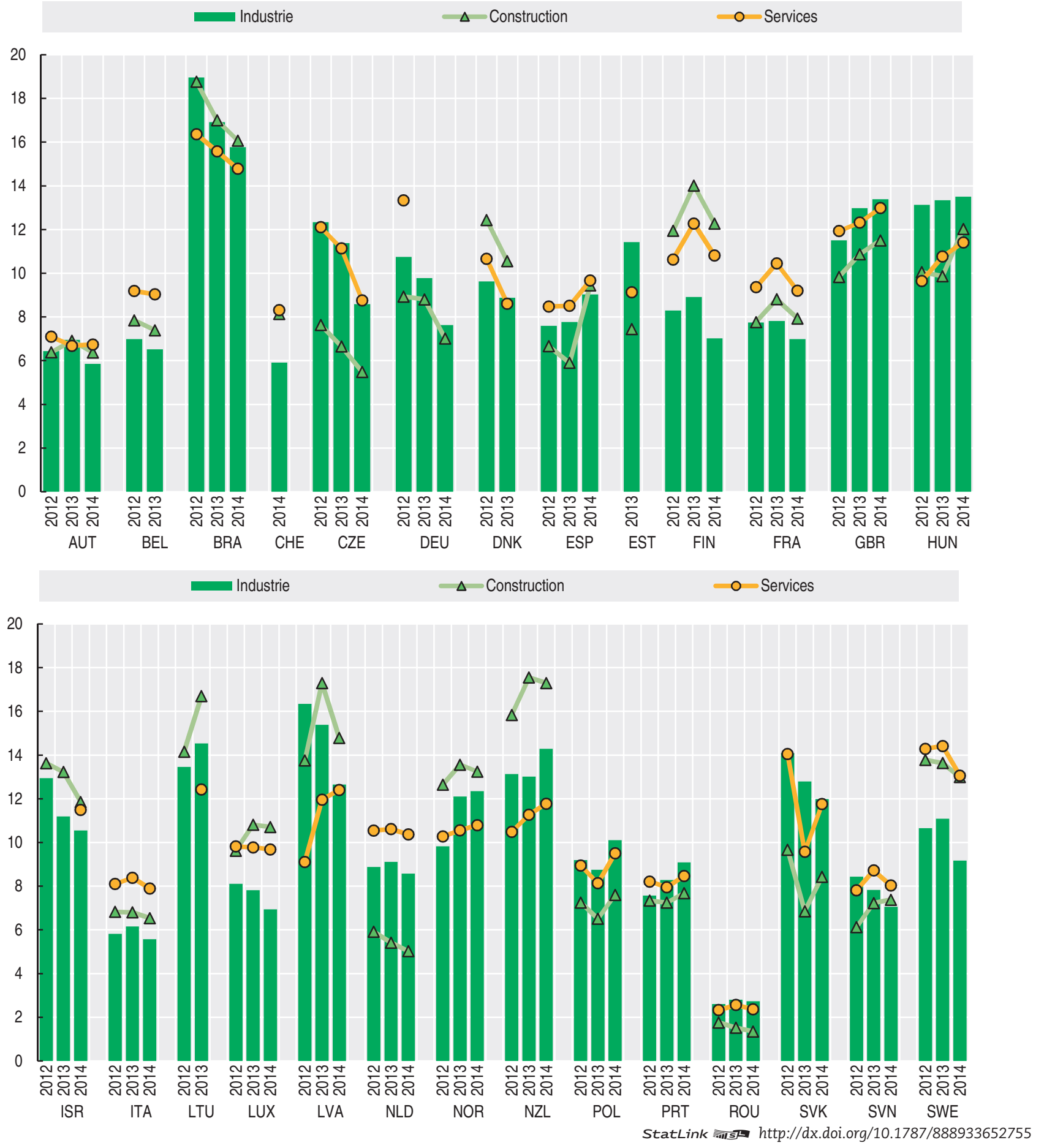
Graphique 4.27. Part de l'emploi dans les entreprises à forte croissance (croissance de l'emploi supérieure à $10 \%$ ) En pourcentage de l'emploi dans les entreprises de dix salariés ou plus, 2014 ou dernière année disponible

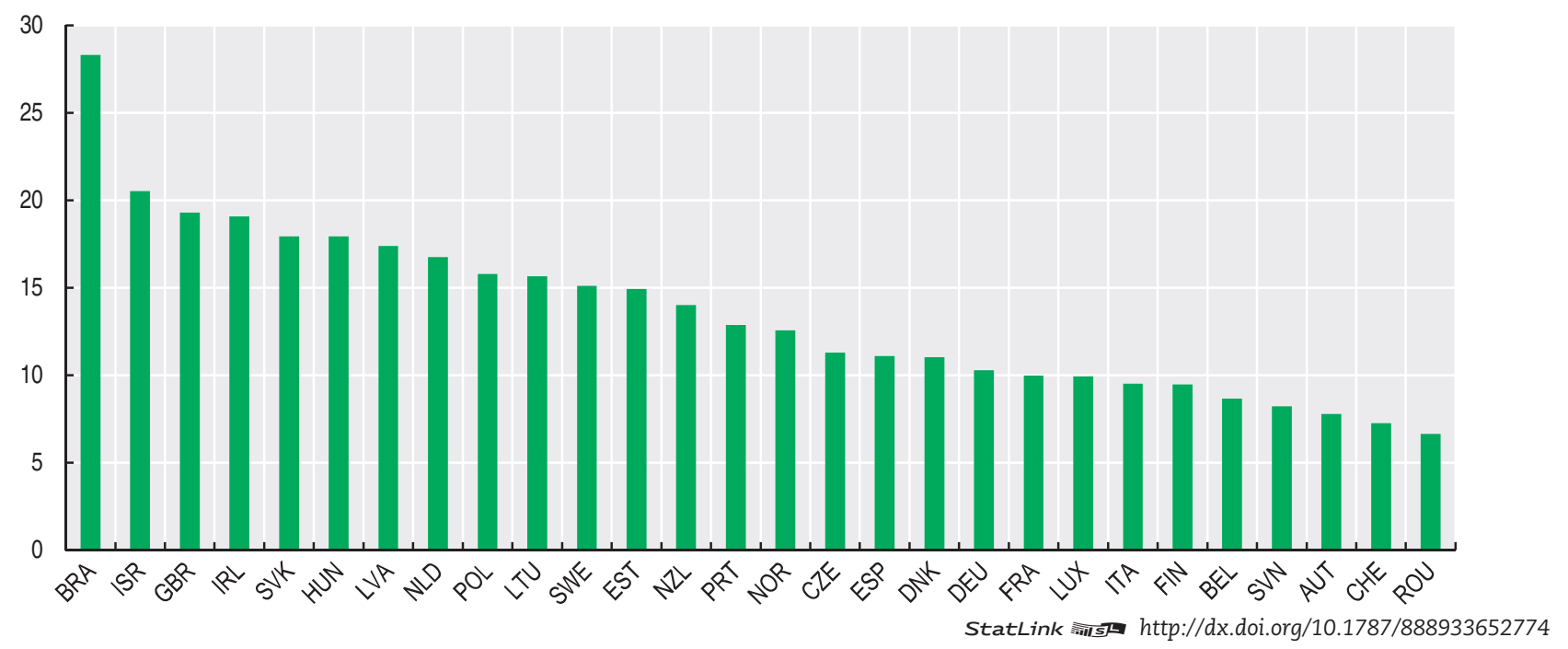

Graphique 4.28. Proportion de gazelles (croissance de l'emploi supérieure à $20 \%$ )

En pourcentage de toutes les entreprises de dix salariés ou plus, par secteur, 2014 ou dernière année disponible

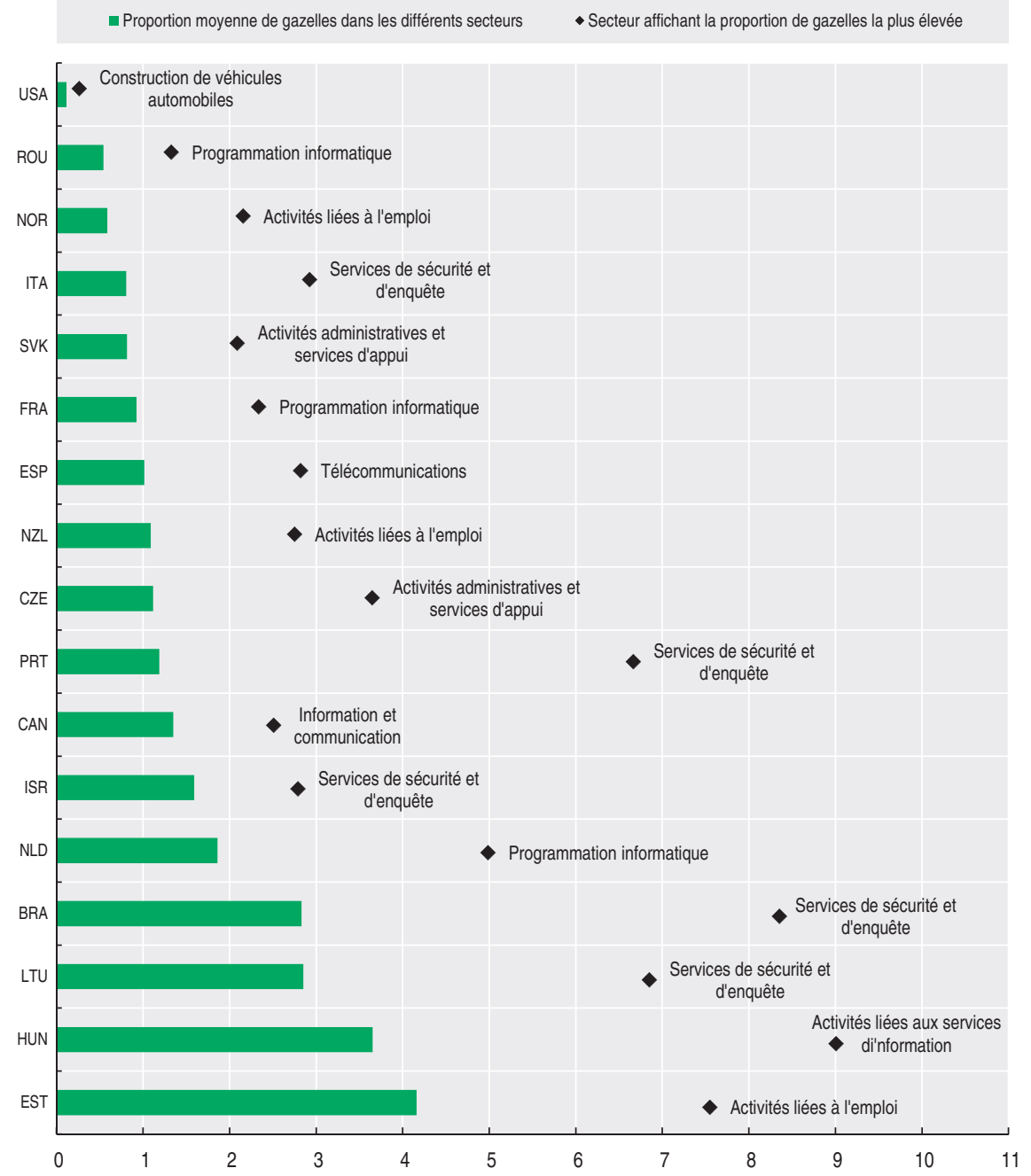




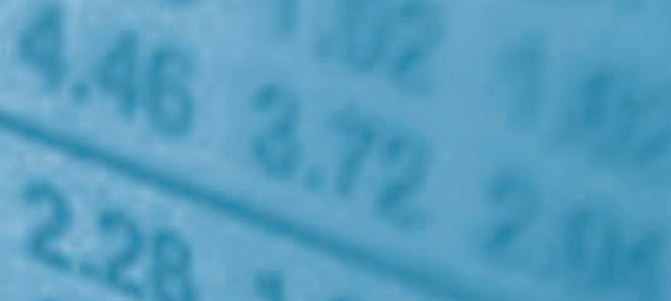

8.09251 .89

10.00 .49 ? 70

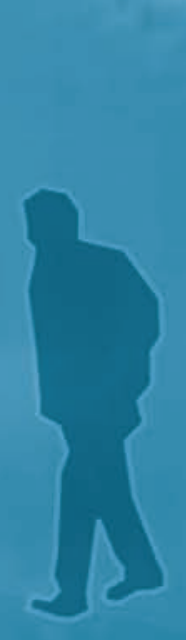$$
7 \times \frac{2.87}{2}
$$

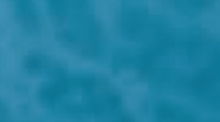

8

4itis

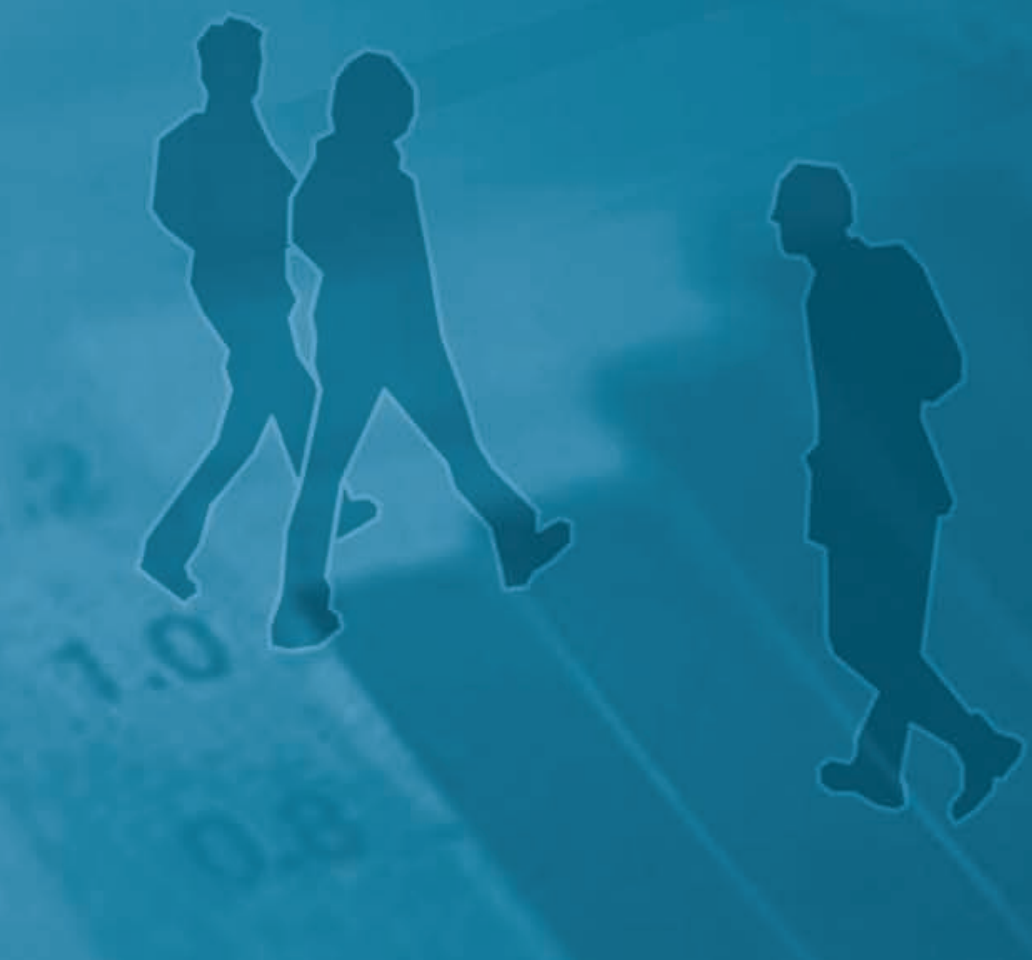

1,0

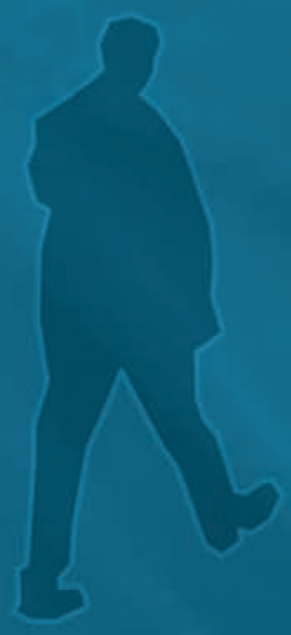

$x^{2}=x^{2}=$

a. 


\section{PME ET COMMERCE INTERNATIONAL}

Concentration des échanges

Échanges par taille d'entreprise

PME et proximité du marché

Échanges par type de contrôle capitalistique des entreprises 


\section{À savoir}

- La proportion d'entreprises participant aux échanges internationaux varie considérablement d'un pays à l'autre, s'établissant entre $10 \%$ et $40 \%$ pour les exportations et entre $10 \%$ et $70 \%$ pour les importations. Ces proportions sont généralement plus élevées dans les petits pays, en raison de la taille limitée du marché intérieur, mais là aussi on observe des écarts importants entre les pays.

- Dans la quasi-totalité des pays et toutes classes de taille confondues, le nombre d'entreprises importatrices est généralement plus élevé que le nombre d'entreprises exportatrices, à l'exception notable des États-Unis où la situation est inverse pour toutes les catégories de PME.

- Il existe des écarts importants entre les PME de tailles différentes en ce qui concerne leur participation aux échanges internationaux. En moyenne, $15 \%$ des microentreprises commercent avec l'étranger, contre $60 \%$ pour les petites entreprises et $80 \%$ pour les entreprises de taille moyenne.

- Les 100 premières entreprises exportatrices représentent une part significative des exportations dans tous les pays, allant d'environ un quart environ en Italie à plus de $90 \%$ au Luxembourg.

\section{Pertinence}

La fragmentation internationale de la production a alimenté l'essor des chaînes de valeur mondiales au cours des dernières décennies, et s'est accompagnée d'une augmentation des échanges de biens intermédiaires. Des différences persistent toutefois d'un pays à l'autre en termes de niveau d'intégration, en particulier pour les PME, et de pénétration du ou des marché(s). Une présence sur divers marchés indique bien souvent l'existence d'avantages comparatifs et une résistance aux chocs affectant la demande.

\section{Définitions}

L'utilisation de statistiques de sources douanières sur les échanges de biens vise à saisir tout mouvement de marchandises à la frontière d'un pays, dans le sens sortant (exportations) et entrant (importations). Cette méthode mesure les flux physiques bidirectionnels de marchandises traversant la frontière, conformément à la norme internationale définie dans les "Statistiques du commerce international de marchandises : Concepts et définitions, 2010 » des Nations Unies (New York, 2010).

Les notions clés de cette méthode sont les suivantes : s'agissant des exportations, la destination finale connue d'une marchandise exportée détermine le partenaire commercial de l'entreprise exportatrice; en ce qui concerne les importations, le pays où la marchandise est extraite, produite ou a subi sa dernière transformation (autrement dit, le pays d'origine) détermine le partenaire commercial de l'entreprise importatrice.

Les statistiques classiques du commerce international décrivent les flux commerciaux entre les pays en les ventilant par types de biens et de services. Les données issues de la base de données de l'OCDE du Commerce par caractéristiques d'entreprise (CCE) utilisent comme critère de ventilation les caractéristiques des entreprises actrices du commerce international.
La proportion d'exportateurs (d'importateurs) correspond au nombre d'entreprises exportatrices (importatrices) rapporté au nombre total d'entreprises. La proportion d'exportateursimportateurs correspond au nombre d'entreprises qui sont à la fois exportatrices et importatrices parmi les entreprises exportatrices ou importatrices.

La concentration des exportations parmi les entreprises exportatrices correspond au rapport entre la valeur des exportations par tranche (les 10 premières entreprises exportatrices, de la $11^{\mathrm{e}}$ à la $50^{\mathrm{e}}$, et de la $51^{\mathrm{e}}$ à la $100^{\mathrm{e}}$ ) et la valeur totale des exportations.

Le pourcentage de la valeur des exportations vers $x$ pays partenaires est le ratio de la valeur des exportations par entreprise ayant x pays partenaires rapportée à la valeur totale des exportations.

\section{Comparabilité}

Les données qui reflètent uniquement les circuits d'exportation (et d'importation) directs peuvent conduire à sous-estimer le véritable niveau d'intégration au sein des chaînes de valeur mondiales, en particulier par taille d'entreprise. À titre d'exemple, des PME situées en amont produisant des biens et services intermédiaires peuvent participer aux chaînes de valeur mondiales en fournissant de grandes entreprises exportatrices. De même, un certain nombre de petites entreprises peuvent exporter (et importer) par l'intermédiaire de grossistes.

Il n'est pas toujours possible de trouver, pour chaque entreprise, une concordance entre les registres de commerce et les registres d'entreprises. En règle générale, les défauts de concordance concernent des petites entreprises, comme le suggère les faibles valeurs moyennes des échanges de ces entreprises non ventilées. Pour cette raison, les graphiques 5.1 et 5.2 incluent toutes les entreprises non ventilées dans la population des PME, ainsi que la valeur de leurs échanges.

Les données présentées dans les graphiques 5.1, 5.2 et 5.4 proviennent de deux bases de données de l'OCDE : la base de données des Statistiques structurelles et démographiques des entreprises et la base de données du Commerce par caractéristiques d'entreprise. La couverture des entreprises dans ces deux bases de données peut ne pas coïncider si différents seuils sont pris en compte ou si des unités statistiques différentes sont utilisées pour enregistrer le nombre d'entreprises.

\section{Sources}

Statistiques structurelles et démographiques des entreprises (base de données de l'OCDE), http://dx.doi.org/10.1787/sdbs-data-fr.

Base de données de l'OCDE sur le commerce international par caractéristiques économiques des entreprises (CCE), http://stats. oecd.org/Index.aspx?DataSetCode=TEC1_REV4.

\section{Pour en savoir plus}

Conseil nordique des Ministres et OCDE (2016), Nordic Countries in Global Value Chains 2016, http://www.dst.dk/Site/Dst/ Udgivelser/GetPubFile.aspx?id=28140\&sid=nordglobchains.

OCDE et Groupe de la Banque mondiale (2015) « Inclusive Global Value Chains. Policy options in trade and complementary areas for GVC: Integration by small and medium enterprises and low-income developing countries ", https://www.oecd. org/trade/OECD-WBG-g20-guc-report-2015.pdf. 
Graphique 5.1. Proportion d'exportateurs, industrie

Part des entreprises exportatrices dans le nombre total d'entreprises

Exportateurs 2014

Exportateurs 2011

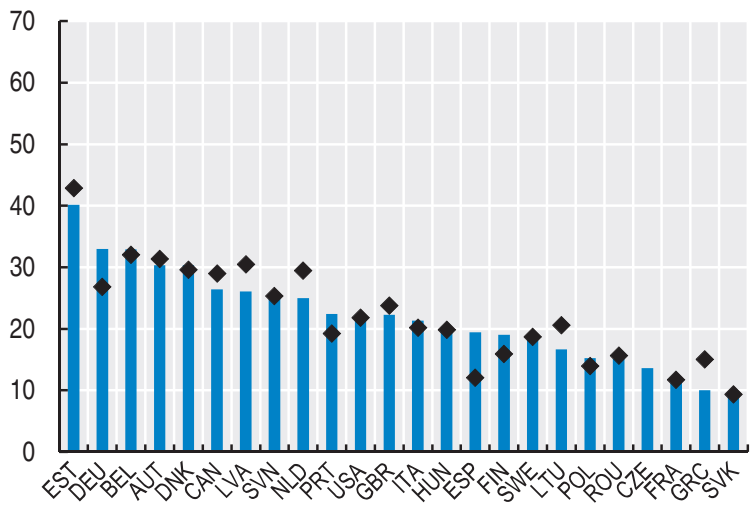

Part des entreprises exportatrices, par taille, 2014
- PME
Grandes entreprises

- PME 2011

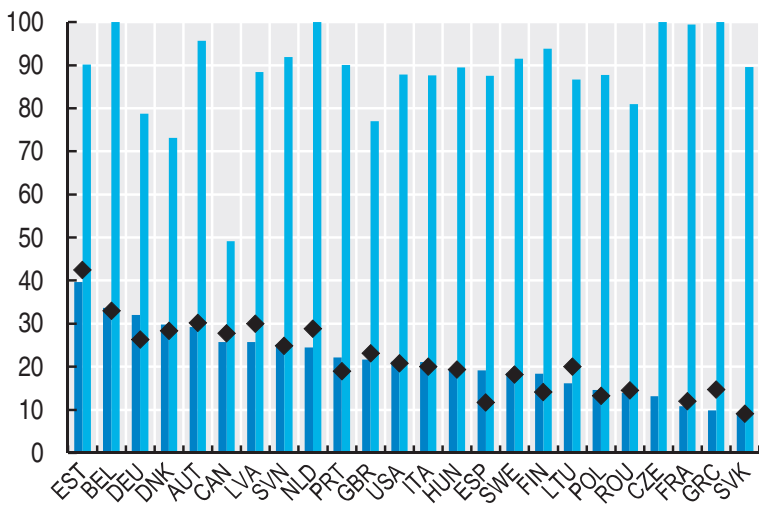

StatLink -illsta $h t t p: / / d x . d o i . o r g / 10.1787 / 888933652793$

Graphique 5.2. Proportion d'importateurs, industrie

Part des entreprises importatrices dans le nombre total d'entreprises

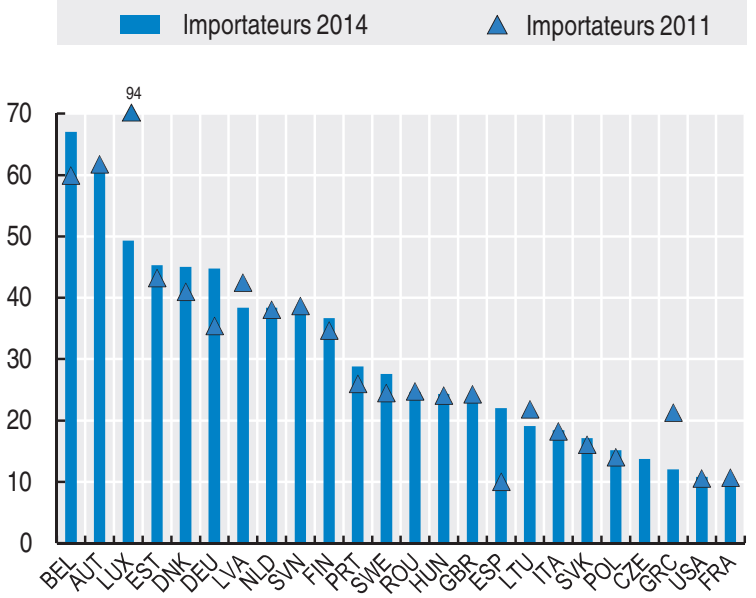

Part des entreprises importatrices, par taille, 2014

$$
\text { PME Grandes entreprises }
$$

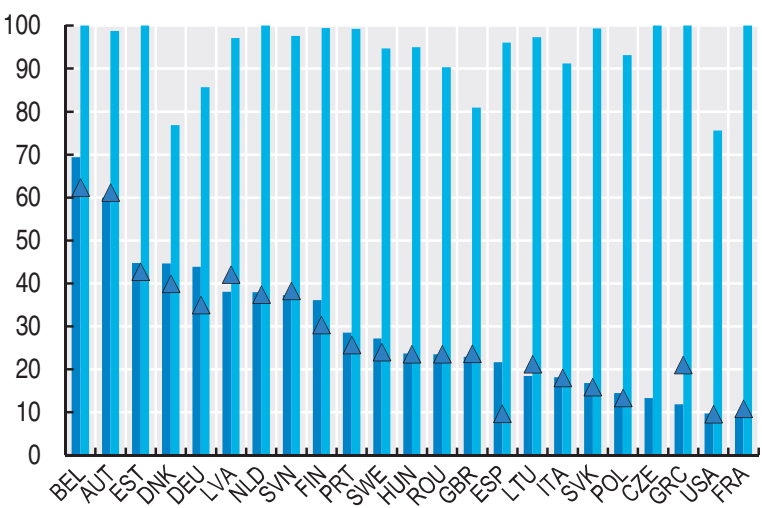

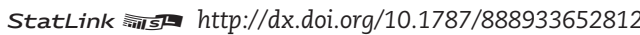

Graphique 5.3. Proportion d'exportateurs-importateurs, industrie

Part des entreprises à la fois exportatrices et importatrices dans le nombre total d'entreprises exportatrices (importatrices), 2015 ou dernière année disponible

Exportateurs

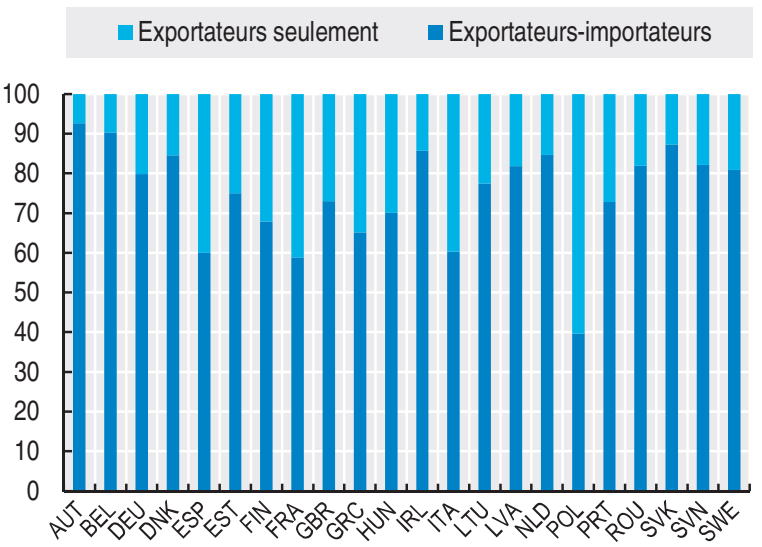

Importateurs

- Importateurs seulement $\quad$ Exportateurs-importateurs

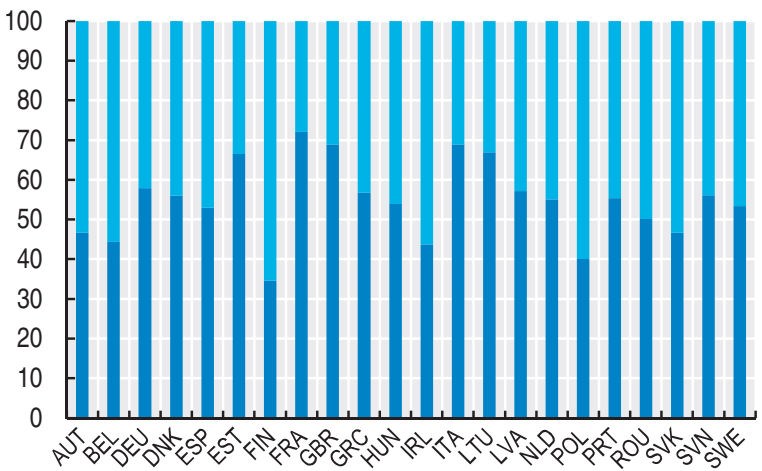

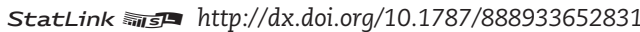




\section{PME ET COMMERCE INTERNATIONAL}

\section{Concentration des échanges}

Graphique 5.4. Proportion de PME actrices du commerce international, par taille, industrie En pourcentage de l'ensemble des entreprises, 2014 ou dernière année disponible
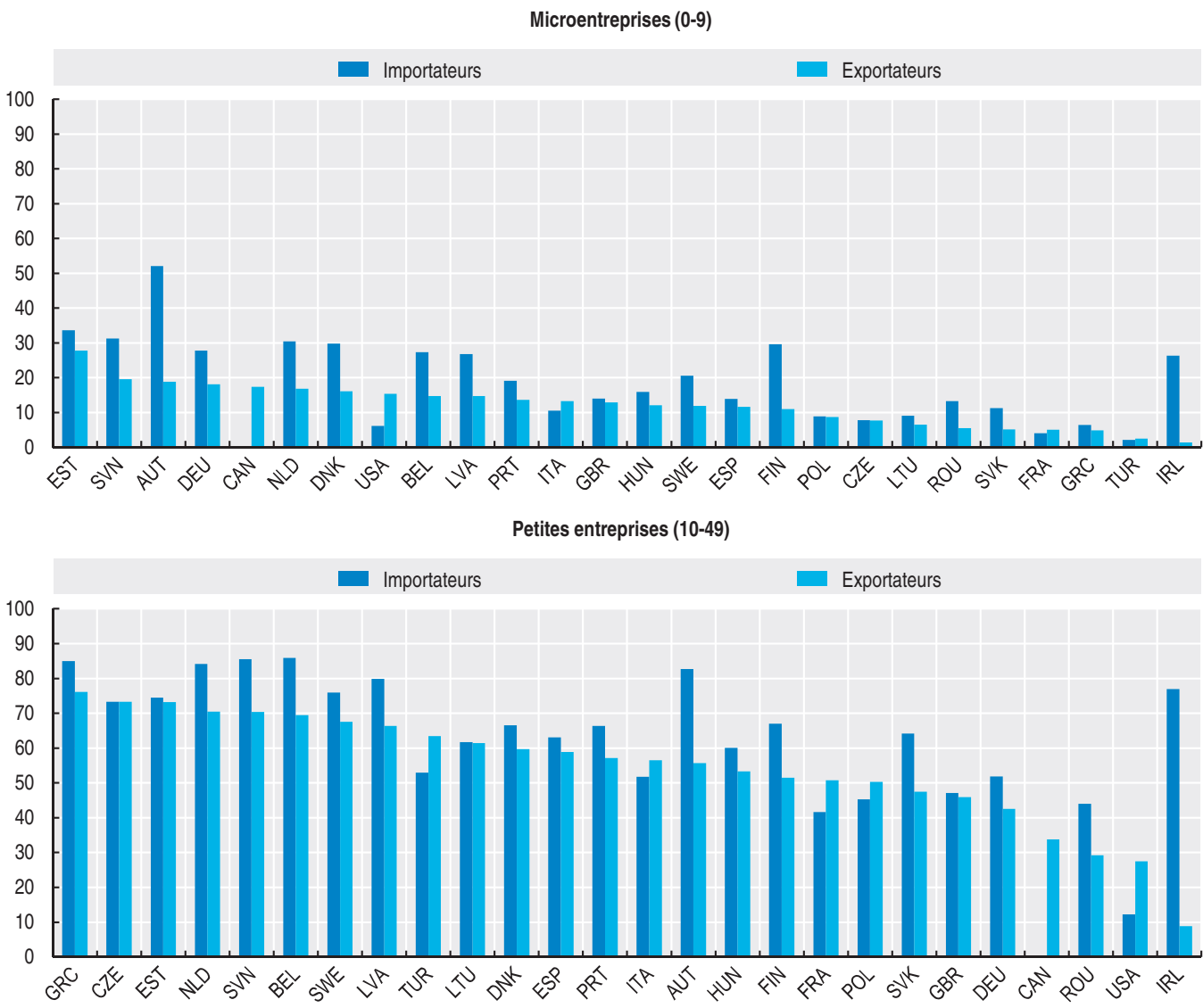

Moyennses entreprises (50-249)

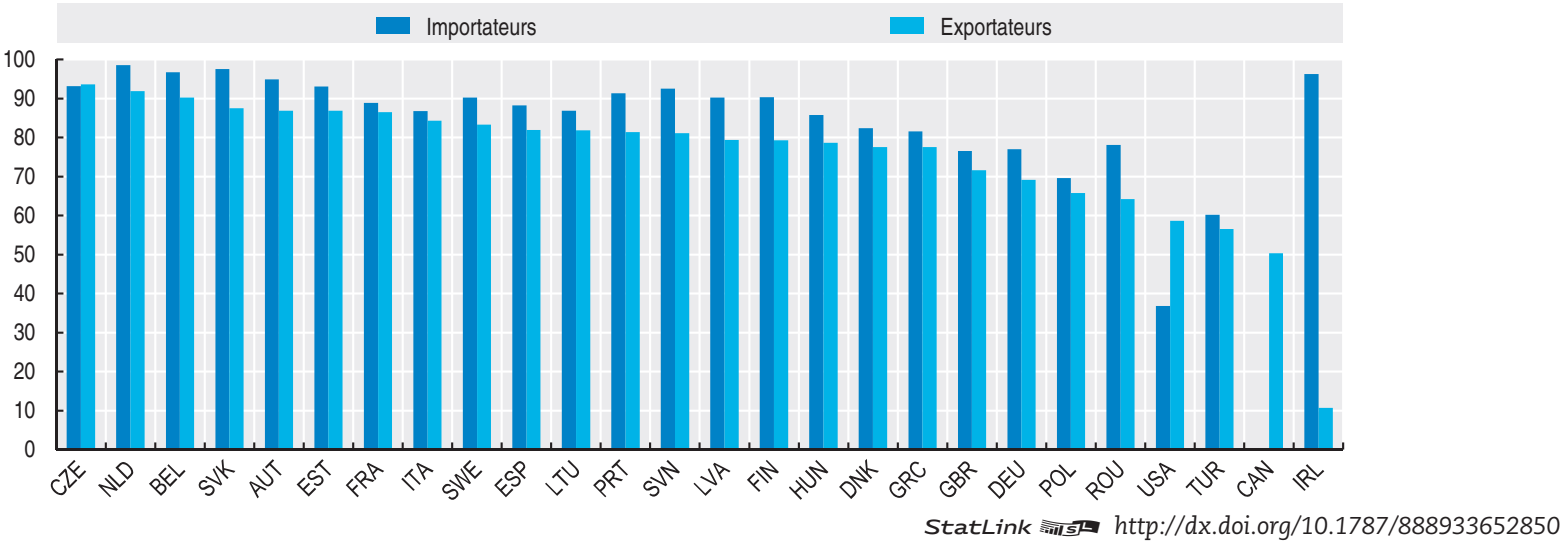


Graphique 5.5. Concentration des exportations par classe d'entreprises exportatrices, ensemble de l'économie En pourcentage de la valeur totale des exportations, 2015 ou dernière année disponible

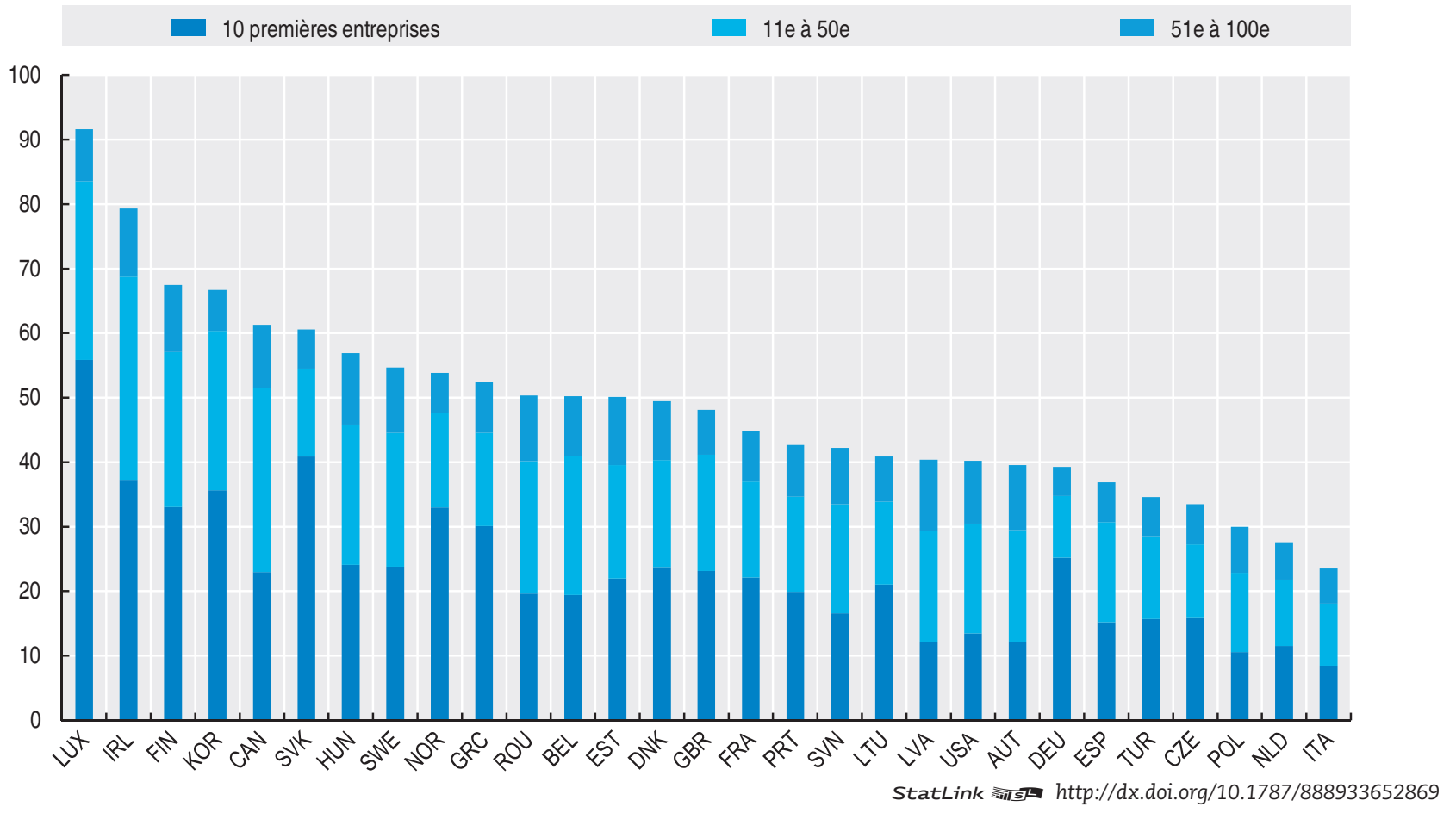

Graphique 5.6. Concentration de la valeur des exportations par nombre de partenaires commerciaux, ensemble de l'économie

En pourcentage de la valeur totale des exportations, 2015 ou dernière année disponible

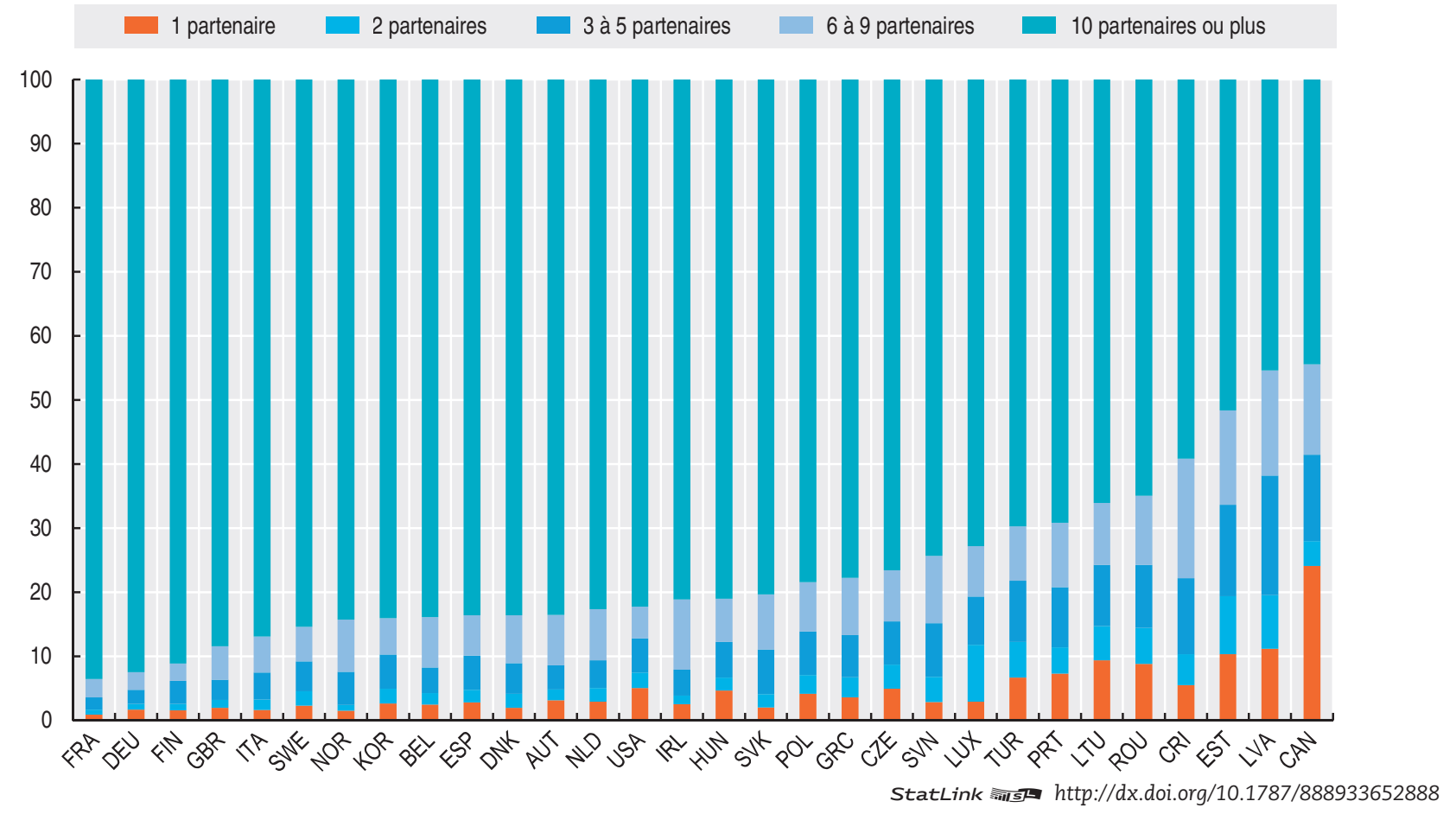




\section{À savoir}

- Alors que les microentreprises, c'est-à-dire des entreprises comptant moins de 10 salariés, représentent entre $25 \%$ et $70 \%$ des entreprises exportatrices, elles ne contribuent que de façon modeste aux exportations totales en valeur.

- Dans le secteur du commerce de gros et de détail, les microentreprises sont l'un des principaux moteurs du commerce international. Elles représentent environs les trois-quarts de l'ensemble des entreprises de ce secteur actives à l'international, et entre $15 \%$ et $50 \%$ de l'ensemble des importations et exportations de ce secteur.

- En règle générale, le ratio des exportations (importations) sur le chiffre d'affaires total est d'autant plus élevé que l'entreprise est grande et l'économie petite. Au sein de l'OCDE, c'est en Irlande et aux Pays-Bas, deux pays qui abritent de nombreuses entreprises multinationales étrangères à forte intensité de droits de propriété intellectuelle, que le ratio des exportations sur le chiffre d'affaires est le plus haut.

\section{Pertinence}

Les écarts constatés dans la participation des entreprises aux échanges en fonction de leur taille et des pays peuvent être le signe d'importants obstacles à l'intégration dans le commerce international, en particulier pour les petites entreprises, et, par voie de conséquence, appeler l'attention sur la nécessité d'examiner les canaux indirects d'intégration au sein des chaînes de valeur mondiales.

\section{Définitions}

La proportion d'exportateurs (importateurs) par taille d'entreprise est définie comme le rapport entre le nombre d'exportateurs (importateurs) dans chaque classe de taille et le nombre total d'exportateurs (importateurs).

La part d'exportations (ou importations) par taille d'entreprise désigne la valeur des exportations (importations) pour chaque classe de taille rapportées à la valeur totale des exportations (ou importations).

Le ratio des exportations (importations) sur le chiffre d'affaires représente la part de la valeur des exportations (importations) des entreprises exportatrices (importatrices) dans le chiffre d'affaires total de l'ensemble des entreprises.

La valeur moyenne des exportations (importations) par entreprise est calculée en divisant la valeur des exportations (importations) par le nombre d'entreprises exportatrices (importatrices). salaires et autres cotisations patronales.

\section{Comparabilité}

Il n'est pas toujours possible de trouver, pour chaque entreprise, une concordance entre les registres de commerce et les registres d'entreprises. En règle générale, les défauts de concordance concernent des petites entreprises, comme le suggère les faibles valeurs moyennes des échanges de ces entreprises non ventilées. Pour cette raison, les graphiques 5.8 et 5.10 incluent toutes les entreprises non ventilées dans la population des PME, ainsi que la valeur de leurs échanges.

Les données qui reflètent uniquement les circuits d'exportation (d'importation) directs peuvent conduire à sous-estimer le véritable niveau d'intégration au sein des chaînes de valeur mondiales, en particulier par taille d'entreprise. À titre d'exemple, des PME situées en amont produisant des biens et services intermédiaires peuvent participer aux chaînes de valeur mondiales en fournissant de grandes entreprises exportatrices. De même, un certain nombre de petites entreprises peuvent exporter (et importer) par l'intermédiaire de grossistes.

Les données présentées dans les graphiques 5.8 et 5.10 proviennent de deux bases de données de l'OCDE : la base de données des Statistiques structurelles et démographiques des entreprises et la base de données du Commerce par caractéristiques d'entreprise. La couverture des entreprises dans ces deux bases de données peut ne pas coïncider si différents seuils sont pris en compte ou si des unités statistiques différentes sont utilisées pour enregistrer le nombre d'entreprises.

\section{Sources}

Statistiques structurelles et démographiques des entreprises (base de données de l'OCDE), http://dx.doi.org/10.1787/sdbs-data-fr.

Base de données de l'OCDE sur le commerce international par caractéristiques économiques des entreprises (CCE), http://stats. oecd.org/Index.aspx?DataSetCode=TEC1_REV4.

\section{Pour en savoir plus}

OCDE (2016), "Who's Who in International Trade: A Spotlight on OECD Trade by Enterprise Characteristics data ", OECD Insights Blog, http://oecdinsights.org/2016/04/25/statistical-insightswhos-who-in-international-trade-a-spotlight-on-oecd-trade-byenterprise-characteristics-data/.

OCDE (2009), " Top Barriers and Drivers to SME Internationalisation ", rapport du Groupe de travail de l'OCDE sur les petites et moyennes entreprises (PME) et l'entrepreneuriat, OCDE, https://www.oecd.org/cfe/smes/ 43357832.pdf . 
Graphique.5.7. Proportion d'exportateurs et d'importateurs

En pourcentage de l'ensemble des exportateurs (importateurs) et de la valeur totale des exportations (importations), 2015 ou dernière année disponible

Activités de fabrication

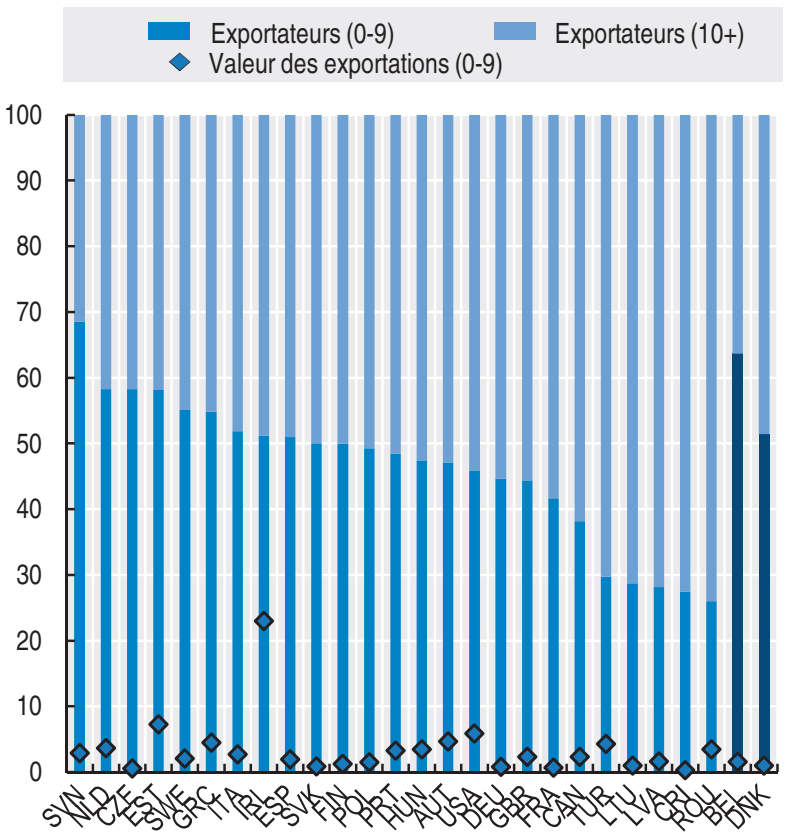

Importateurs (0-9)
$\diamond$ Valeur des importations (0-9)

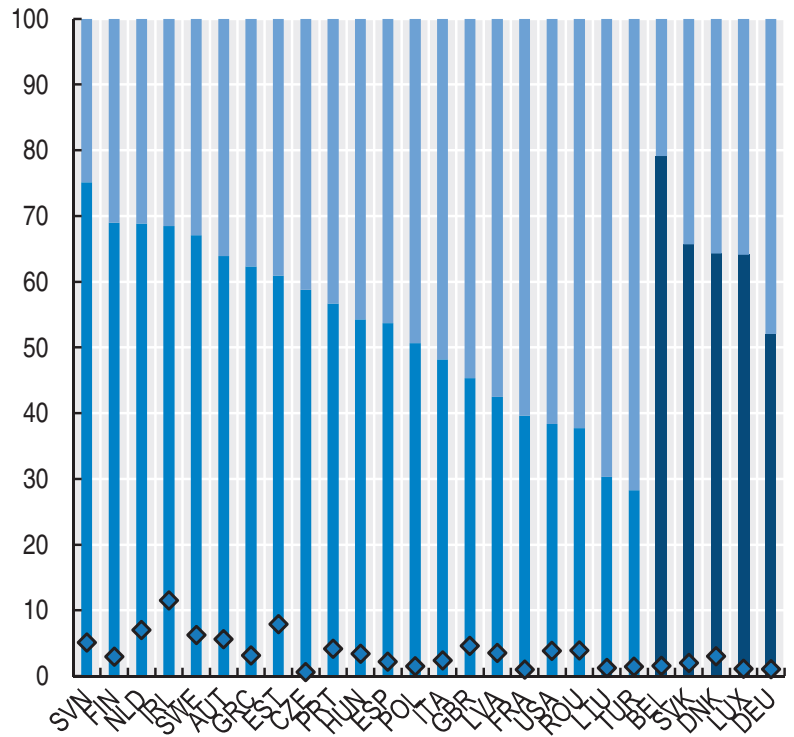

Commerce de gros et de détail

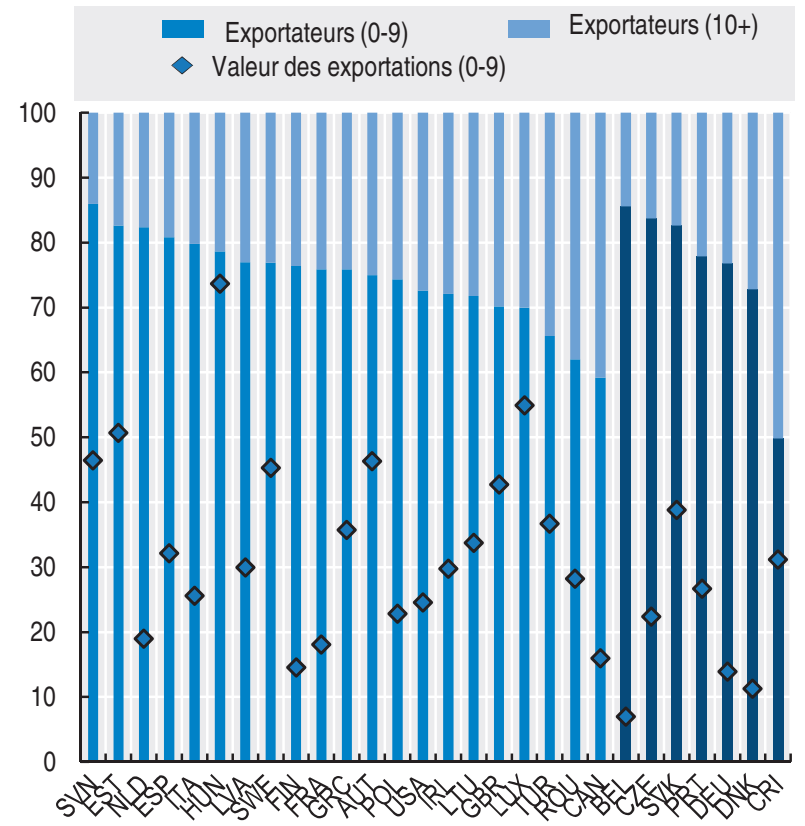
Importateurs (0-9) Importateurs (10+)
$\checkmark$ Valeur des importations (0-9)

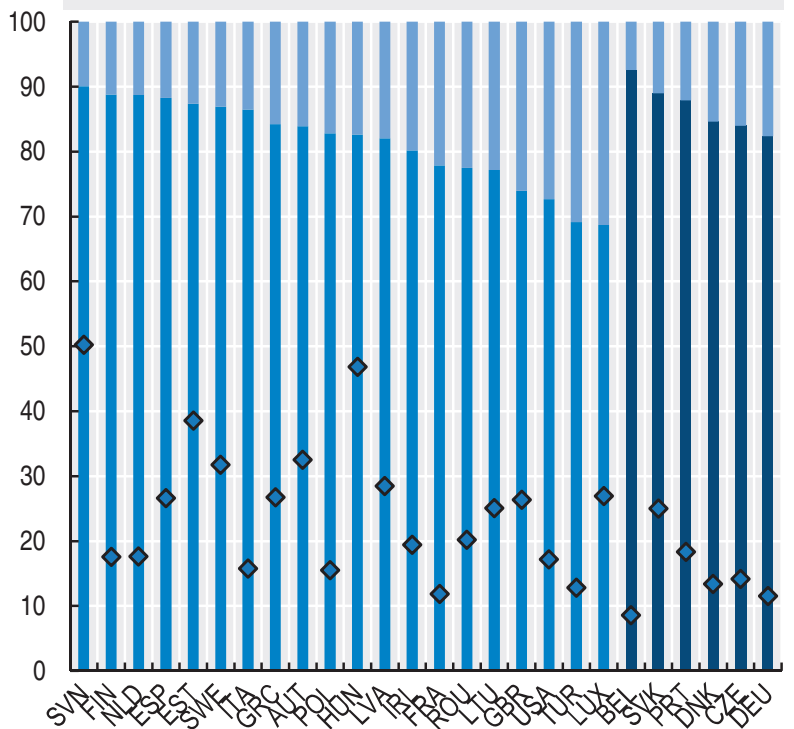

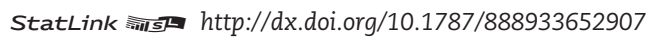




\section{PME ET COMMERCE INTERNATIONAL}

\section{Échanges par taille d'entreprise}

Graphique 5.8. Ratio des exportations sur le chiffre d'affaires, par taille d'entreprise, industrie Valeur des exportations en pourcentage du chiffre d'affaires, 2014 ou dernière année disponible

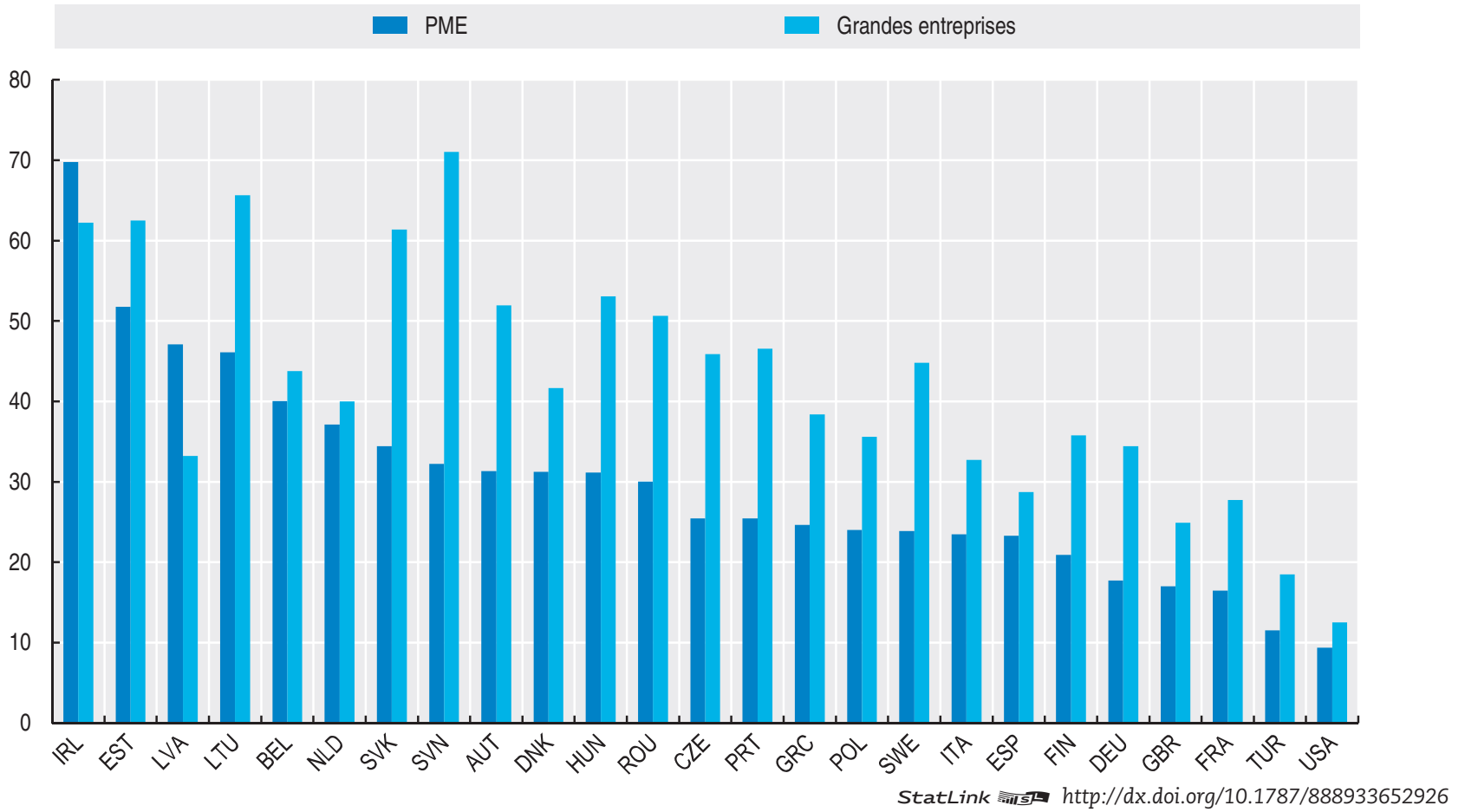

Graphique 5.9. Valeur moyenne des exportations par entreprise, par taille d'entreprise, industrie En millions USD, 2015 ou dernière année disponible

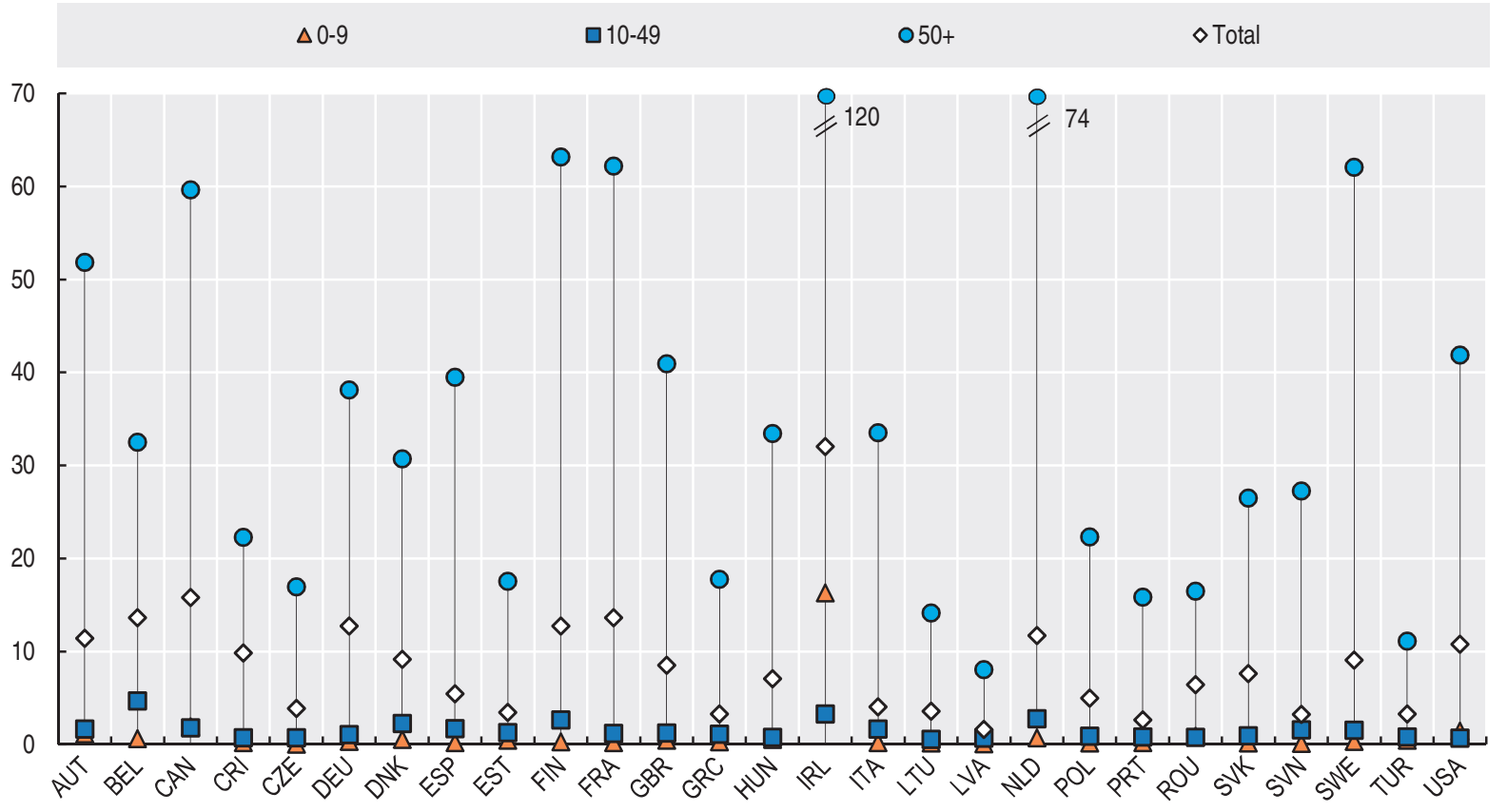

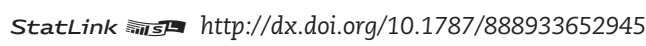


Graphique 5.10. Ratio des importations sur le chiffre d'affaires, par taille d'entreprise, industrie Valeur des importations en pourcentage du chiffre d'affaires, 2014, ou dernière année disponible

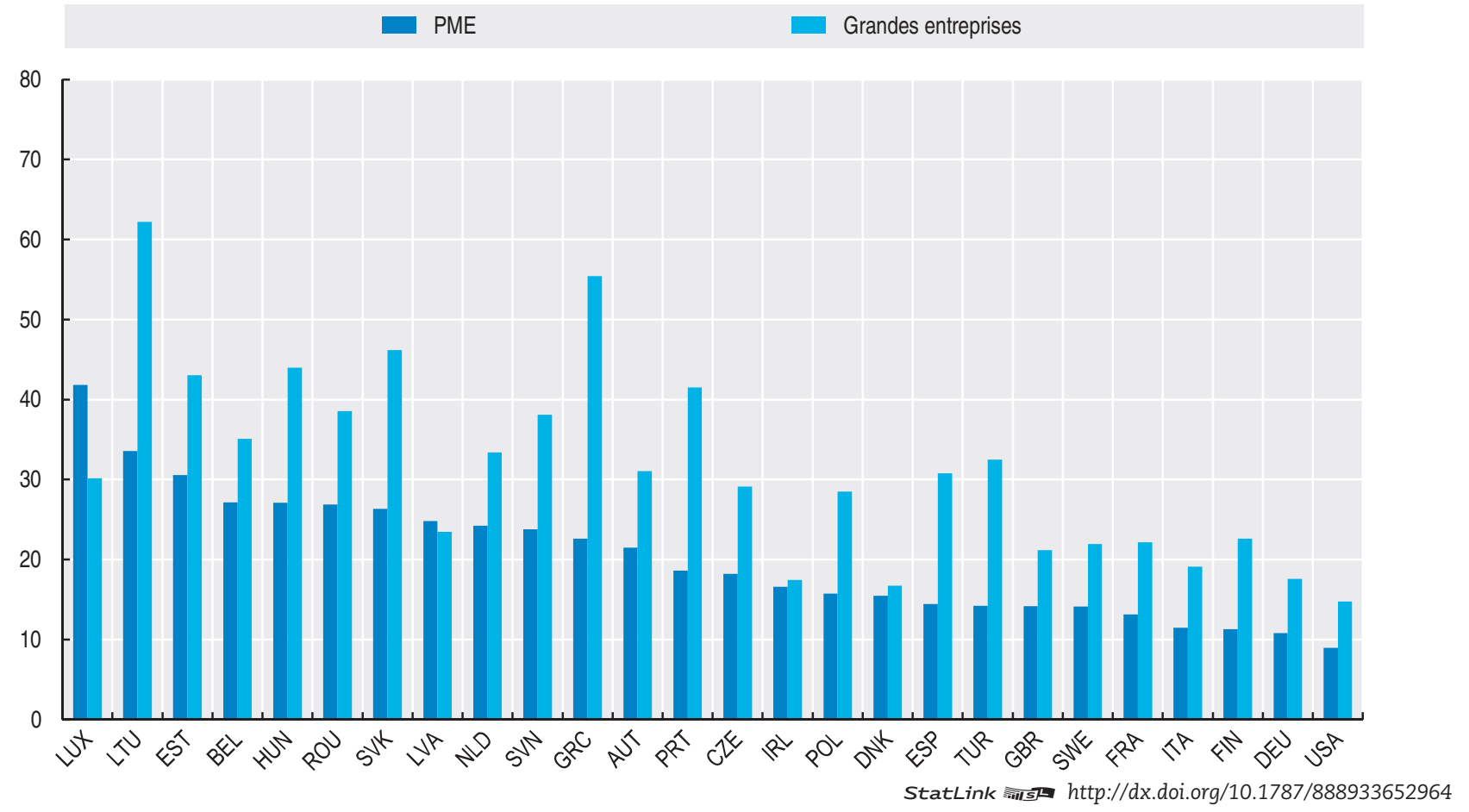

Graphique 5.11. Valeur moyenne des importations par entreprise, par taille d'entreprise, industrie En millions USD, 2015 ou dernière année disponible

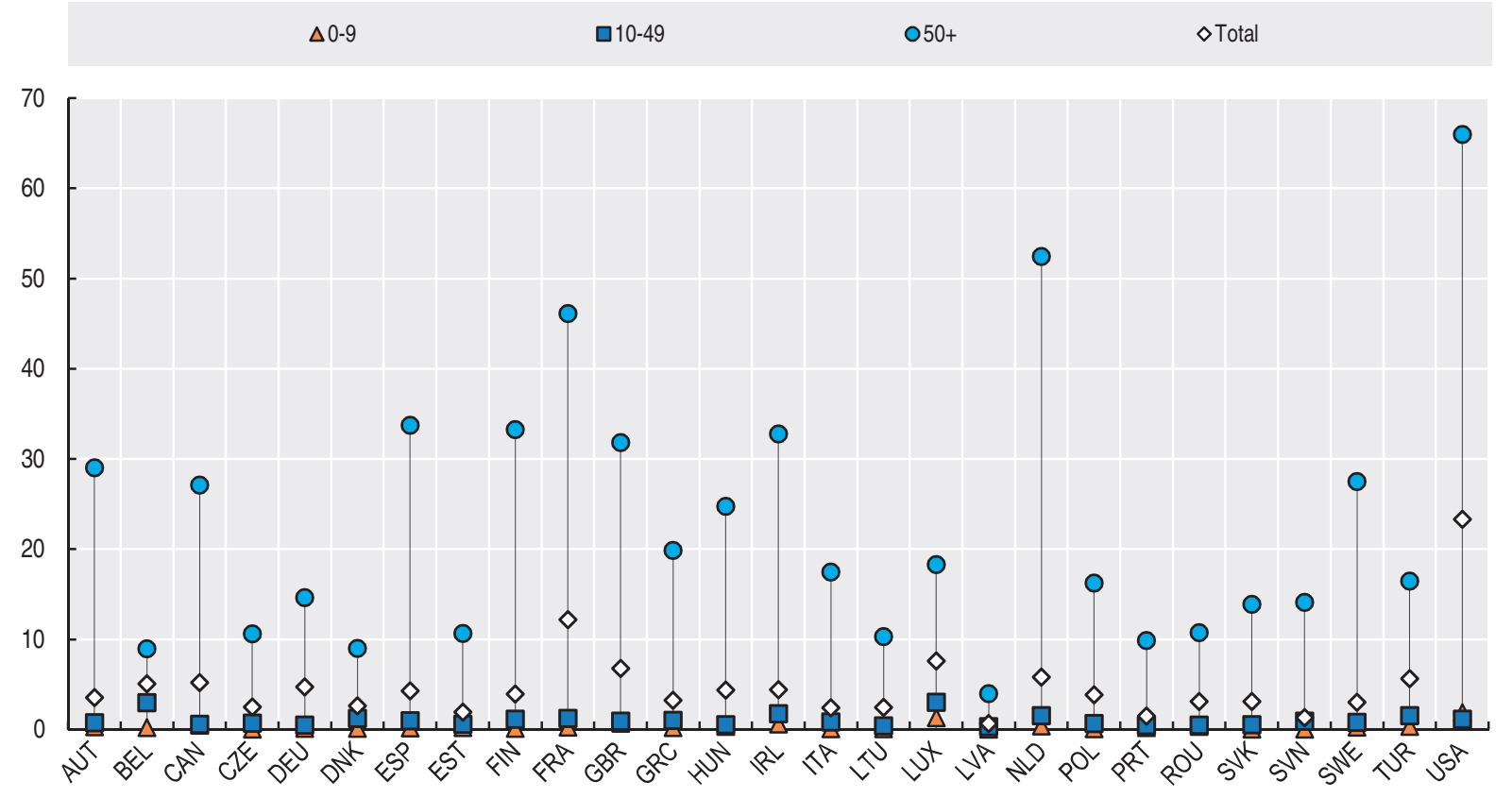

StatLink 亲IS http://dx.doi.org/10.1787/888933652983 


\section{PME et proximité du marché}

\section{À savoir}

- Par rapport aux grandes entreprises, les PME exportent généralement davantage vers des marchés relativement proches de leur pays d'origine - une conséquence des coûts fixes liés à la pénétration de nouveaux marchés, qui ont tendance à être relativement plus élevés pour les petites entreprises. Pour les PME, les obstacles à l'importation semblent être moins lourds que ceux qui pèsent sur les exportations.

- En règle générale, la proportion de PME parmi les entreprises qui exportent vers (ou importent depuis) la Chine et l'Inde est plus faible que la part qu'elles représentent dans le commerce mondial. Cela étant, leur contribution à l'ensemble des exportations à destination de la Chine et de l'Inde est plus élevée au Portugal, en République tchèque et en Turquie, et leur contribution à l'ensemble des importations est plus élevée en Belgique, en Corée, en Lettonie, aux Pays-Bas et au Royaume-Uni.

- Dans tous les pays, la proportion de PME qui importent depuis la Chine est systématiquement plus élevée que celles qui importent depuis l'Inde.

\section{Pertinence}

Les données relatives à la participation aux échanges par pays partenaire et par taille peuvent mettre en évidence d'importants obstacles à l'intégration dans le commerce international, en particulier pour les petites entreprises, et, par voie de conséquence, appeler l'attention sur la nécessité d'examiner les canaux indirects d'intégration au sein des chaînes de valeur mondiales.

\section{Définitions}

La proportion de PME parmi les exportateurs (importateurs) correspond au nombre de PME exportatrices (importatrices) divisé par le nombre total d'entreprises exportatrices (importatrices). La proportion de PME parmi les entreprises qui exportent vers (importent depuis) le pays $x$ est calculé en divisant le nombre de PME qui exportent vers (importent depuis) le pays x par le nombre total d'entreprises qui exportent vers (importent depuis) ce pays.
La part des PME dans les exportations (importations) vers (depuis) le pays $x$ correspond à la valeur des exportations (importations) des PME vers (depuis) le pays $\mathrm{x}$ divisée par les exportations (importations) totales depuis (vers) ce pays.

\section{Comparabilité}

Les données recouvrent tous les secteurs de l'économie.

\section{Sources}

Base de données de l'OCDE sur le commerce international par caractéristiques économiques des entreprises (CCE), http://stats. oecd.org/Index.aspx?DataSetCode=TEC1_REV4.

\section{Pour en savoir plus}

Conseil nordique des Ministres et OCDE (2016), Nordic Countries in Global Value Chains 2016, http://www.dst.dk/Site/Dst/ Udgivelser/GetPubFile.aspx?id=28140\&sid=nordglobchains.

OCDE (2016), “Who's Who in International Trade: A Spotlight on OECD Trade by Enterprise Characteristics data », OECD Insights Blog, http://oecdinsights.org/2016/04/25/statistical-insightswhos-who-in-international-trade-a-spotlight-on-oecd-trade-byenterprise-characteristics-data/.

OCDE (2009), "Top Barriers and Drivers to SME Internationalisation ", rapport du Groupe de travail de l'OCDE sur les petites et moyennes entreprises (PME) et l'entrepreneuriat, OCDE, https://www.oecd.org/cfe/ smes/43357832.pdf. 
Graphique 5.12. Nombre de PME impliquées dans les échanges avec la Chine et l'Inde, ensemble de l'économie En pourcentage, 2014 ou dernière année disponible
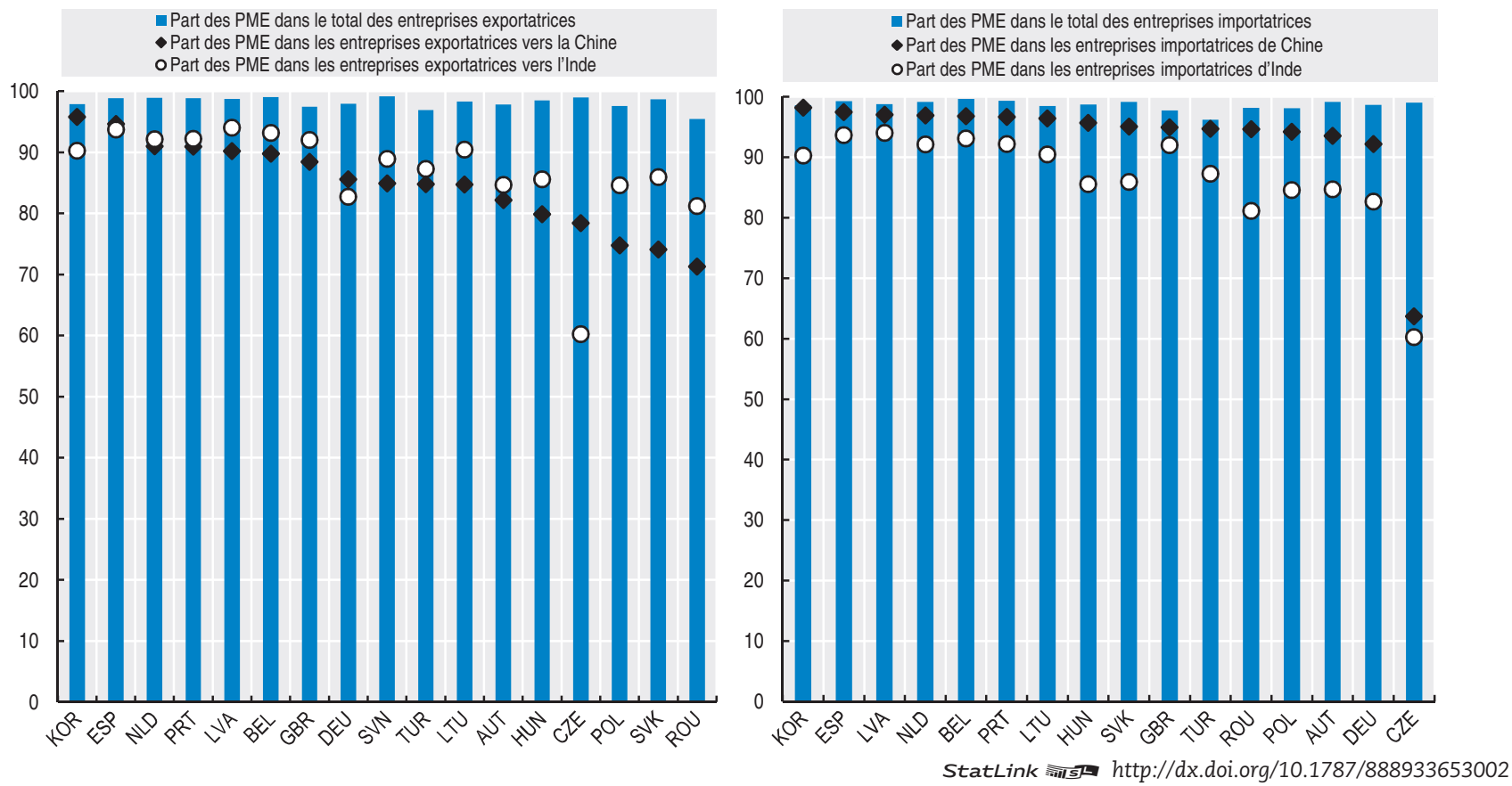

Graphique 5.13. Part des PME dans les échanges avec la Chine et l'Inde, ensemble de l'économie En pourcentage, 2014 ou dernière année disponible
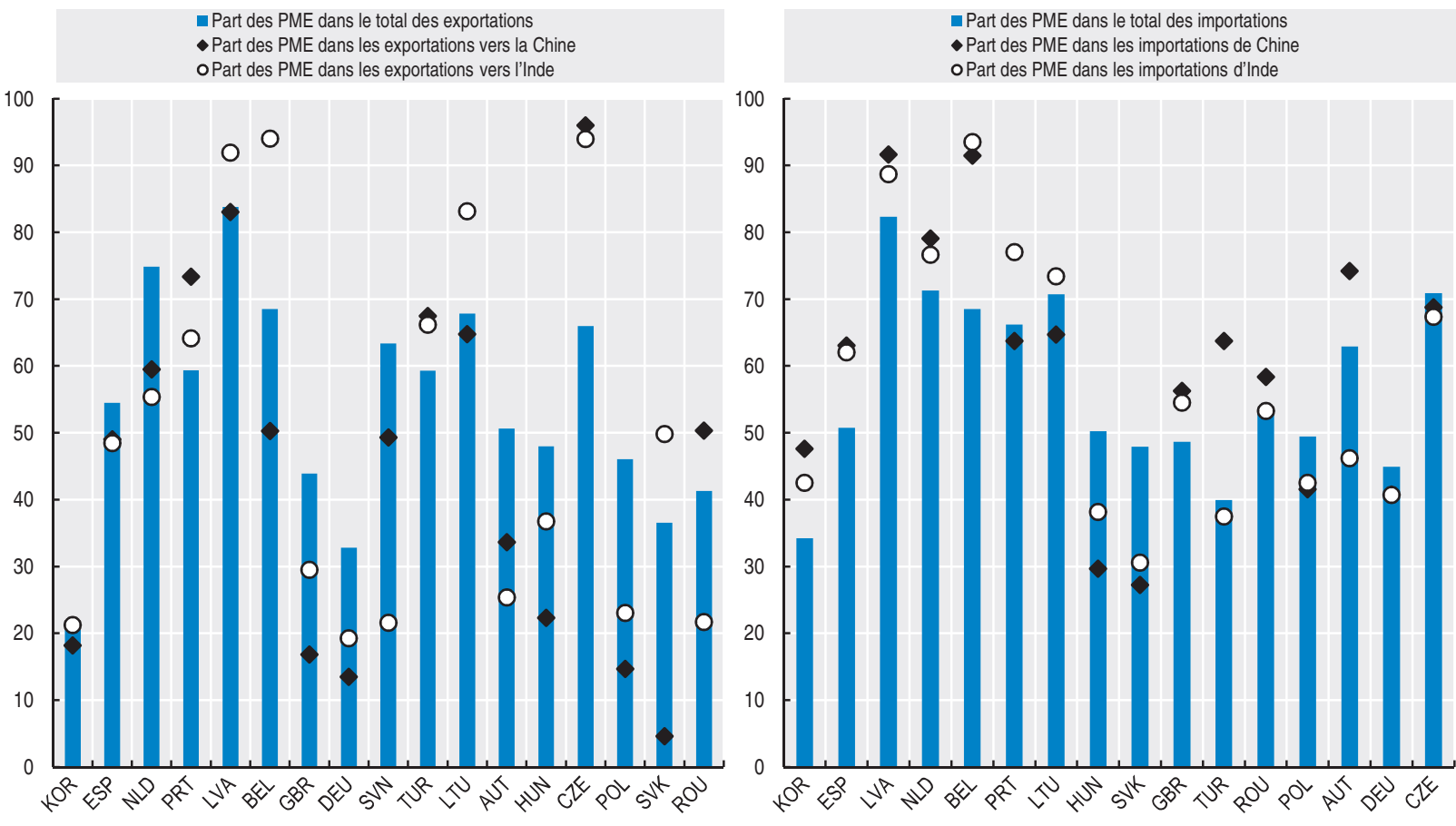

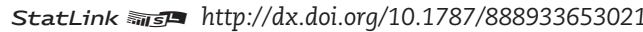




\section{À savoir}

- Les entreprises sous contrôle étranger comptent pour une plus grande part dans les importations et les exportations totales que les entreprises locales. En Hongrie, en République slovaque et en République tchèque, les entreprises exportatrices sous contrôle étranger représentent plus de 80 \% de la valeur totale des exportations et des importations, mais seulement $20 \%$ des entreprises marchandes ; cette tendance est encore plus prononcée en Espagne, en Italie et au Portugal, où moins de $5 \%$ des entreprises sous contrôle étranger représentent une part des importations et des exportations proportionnellement bien plus élevée que ce que l'on pourrait attendre.

- Dans la majorité des pays, les entreprises exportatrices (importatrices) sous contrôle étranger présentent un ratio d'exportations (importations) sur chiffre d'affaires supérieur à celui des entreprises locales.

\section{Pertinence}

Les chaînes de valeur mondiales sont dominées par des entreprises multinationales qui, de plus en plus, répartissent les étapes de la production entre différents lieux en fonction des spécialisations relatives (compétences, accès aux ressources naturelles, infrastructures, environnement réglementaire, etc.) et de l'accès aux marchés, d'où une croissance des échanges de biens intermédiaires proportionnellement plus forte que ce à quoi on pourrait s'attendre. La compréhension de la nature de ces chaînes et de la contribution des filiales étrangères, par leur capital intellectuel ou par la mise en place de chaînes d'approvisionnement locales en amont, est un élément essentiel des stratégies de montée en gamme.

\section{Définitions}

La propriété d'une entreprise est définie en termes de contrôle. La notion de contrôle renvoie à la capacité de disposer d'une majorité au conseil d'administration, de guider les activités de l'entreprise et d'en déterminer la stratégie. Cette capacité est exercée par un investisseur direct unique ou par un groupe d'actionnaires associés agissant de concert et détenant la majorité (plus de $50 \%)$ des actions ordinaires ou des droits de vote. Le contrôle d'une entreprise peut être direct, indirect, immédiat ou ultime.
La part des exportations (importations) des entreprises sous contrôle étranger désigne la valeur des exportations (importations) des entreprises sous contrôle étranger divisée par la valeur totale des exportations.

La proportion d'exportateurs (importateurs) sous contrôle étranger correspond au nombre d'entreprises exportatrices (importatrices) sous contrôle étranger rapporté au nombre total d'entreprises exportatrices (importatrices). Le ratio des exportations (importations) sur le chiffre d'affaires représente la part de la valeur des exportations (importations) des entreprises exportatrices (importatrices) dans le chiffre d'affaires total de l'ensemble des entreprises.

\section{Comparabilité}

Il convient de faire preuve de prudence dans l'interprétation des données. Les données présentées dans cette section proviennent de deux bases de données de l'OCDE : la base de données du Commerce par caractéristiques d'entreprise et la base de données sur l'activité des entreprises multinationales. Or, la couverture des entreprises dans ces deux bases de données peut ne pas coïncider si des seuils différents sont pris en compte ou si des unités statistiques différentes sont utilisées pour enregistrer le nombre d'entreprises.

\section{Sources}

Base de données de l'OCDE sur le commerce international par caractéristiques économiques des entreprises (CCE), http://stats. oecd.org/Index.aspx?DataSetCode=TEC1_REV4.

Base de données de l'OCDE sur les activités des multinationales, http://stats.oecd.org/Index.aspx?DataSetCode=AMNE_IN.

\section{Pour en savoir plus}

OCDE (2016), "Who's Who in International Trade: A Spotlight on OECD Trade by Enterprise Characteristics data », OECD Insights Blog, http://oecdinsights.org/2016/04/25/statistical-insightswhos-who-in-international-trade-a-spotlight-on-oecd-trade-byenterprise-characteristics-data/. 
Graphique 5.14. Proportion d'exportateurs et valeur des exportations, entreprises sous contrôle étranger, industrie

En pourcentage, 2014 ou dernière année disponible - Valeur des exportations $\bullet$ Nombre d'exportateurs

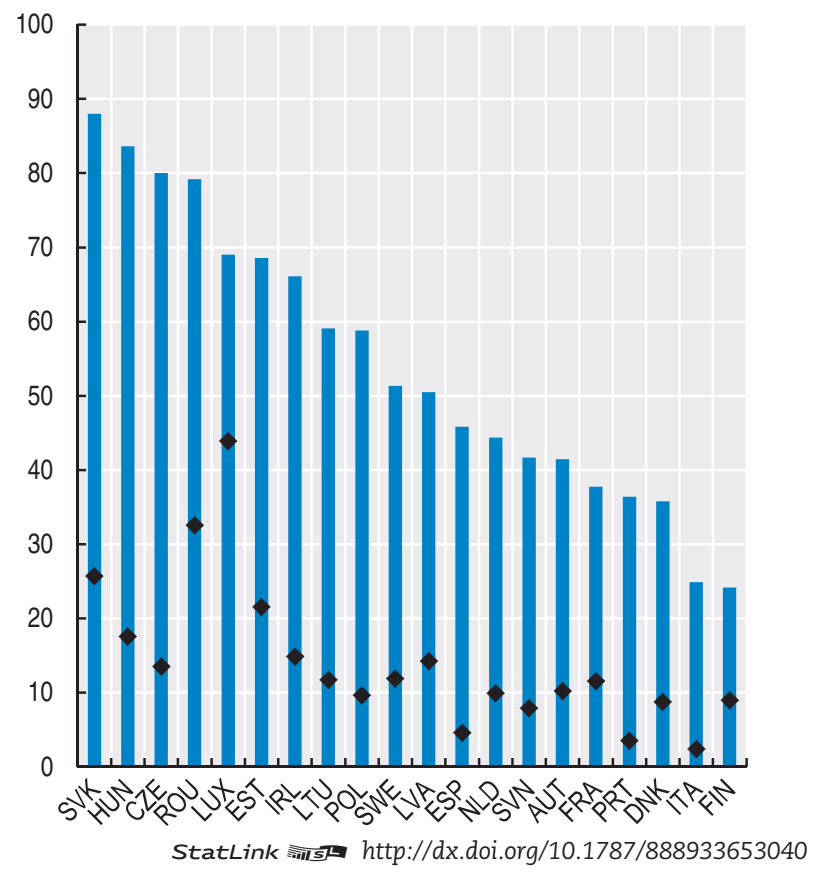

Graphique 5.16. Ratio des exportations sur le chiffre d'affaires par type de contrôle capitalistique, industrie En pourcentage, 2014 ou dernière année disponible

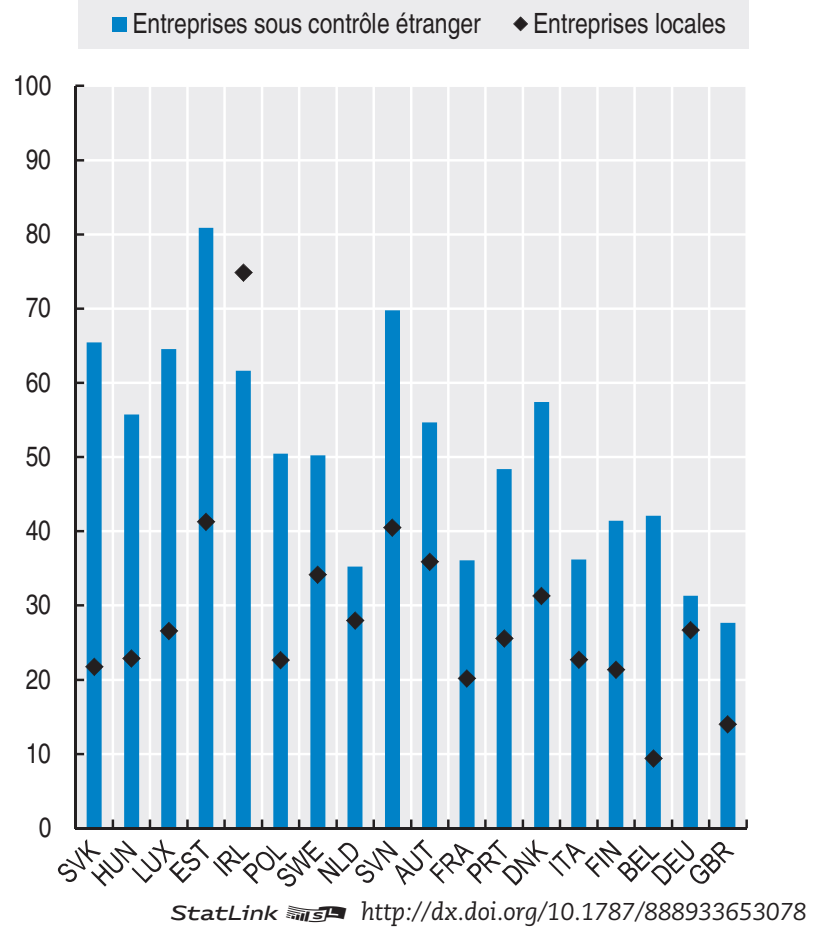

Graphique 5.15. Proportion d'importateurs et valeur des importations, entreprises sous contrôle étranger, industrie

En pourcentage, 2014 ou dernière année disponible

Valeur des importations • Nombre d'importateurs

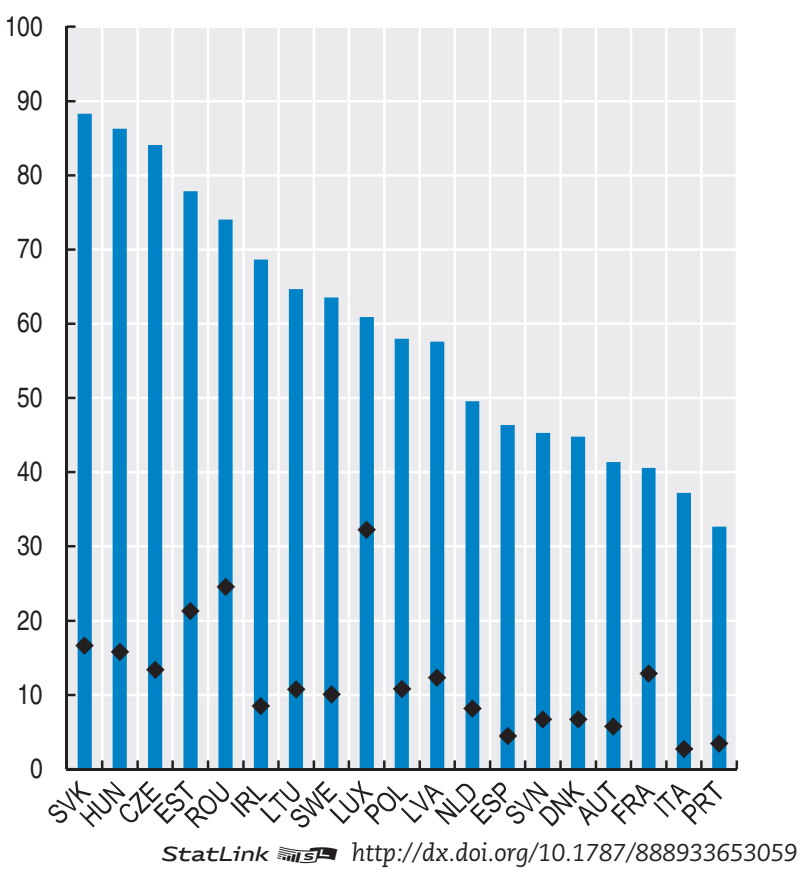

Graphique 5.17. Ratio des importations sur le chiffre d'affaires par type de contrôle capitalistique, industrie En pourcentage, 2014 ou dernière année disponible

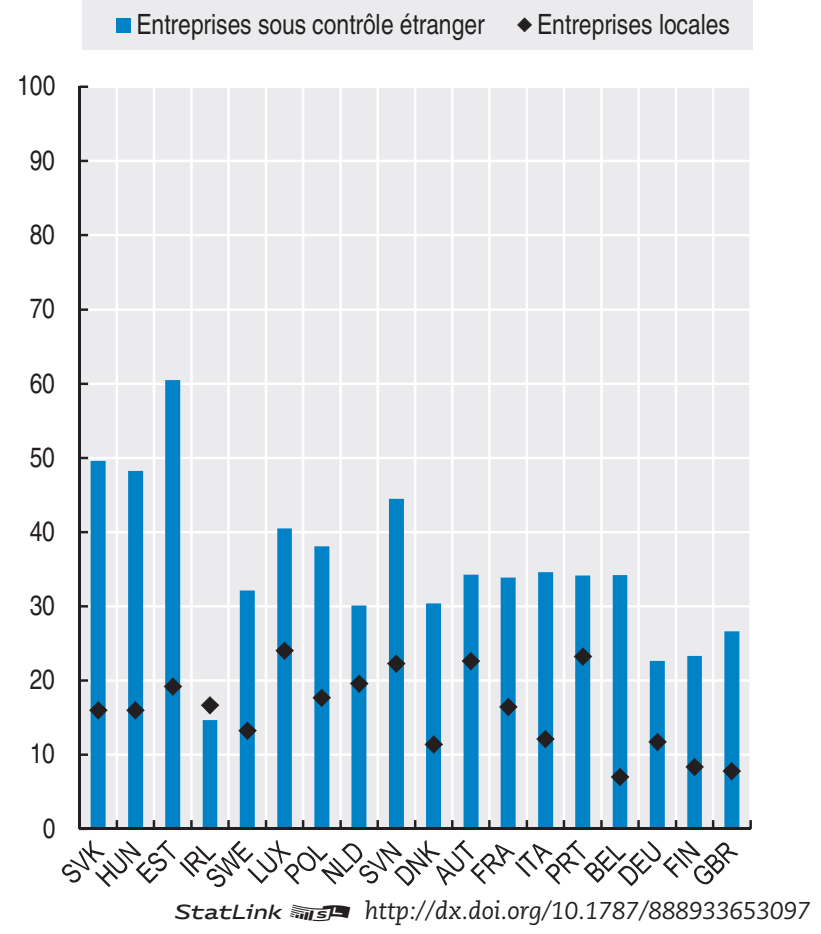





\section{ENTREPRENEURIAT FÉMININ}

Taux de travail indépendant: différences entre femmes et hommes

Revenus du travail indépendant

Attitude à l'égard de l'entrepreneuriat 


\section{À savoir}

- Dans les pays de l'OCDE, une femme occupée sur dix est une travailleuse indépendante, proportion presque inférieure de moitié à celle des hommes $(17 \%)$. Cependant, au cours des dix dernières années, l'écart de taux de travail indépendant entre femmes et hommes s'est réduit dans la quasi-totalité des pays, en particulier en Islande, en Nouvelle-Zélande et en Turquie.

- Dans la majorité des pays, les travailleuses indépendantes exercent principalement dans le secteur des services (70\% ou plus), et ce surtout à leur propre compte plutôt que comme employeuses. La situation est différente chez les hommes travailleurs indépendants, dont une grande part se trouve dans le secteur manufacturier et qui, en règle générale, emploient deux fois et demie plus souvent des salariés que les travailleuses indépendantes.

- Dans tous les pays, on observe aussi un écart entre femmes et hommes chez les jeunes travailleurs indépendants, c'est-à-dire ceux qui ont moins de 30 ans. Si, en 2016, le Chili et le Mexique ont enregistré chez les femmes un taux de travail indépendant légèrement supérieur à celui des hommes, ils étaient les seuls pays dans ce cas.

- La proportion de salariés ayant une seconde activité qu'ils exercent comme travailleurs indépendants a augmenté en 2016 par rapport à 2007 dans la plupart des pays, passant à environ $2 \%$ et $1 \%$ respectivement chez les salariés masculins et féminins.

\section{Pertinence}

L'entrepreneuriat est une source importante de création d'emplois et d'innovation. C'est aussi un moyen de pallier les inégalités, en particulier entre femmes et hommes, où subsistent des disparités non négligeables malgré les possibilités qu'offre le travail indépendant en matière d'articulation de la vie personnelle et de l'activité professionnelle.

\section{Définitions}

Les travailleurs indépendants sont des personnes qui possèdent leur propre entreprise, constituée ou non en société, y travaillent, à leur compte ou non, et déclarent avoir le statut de "travailleur indépendant » lors des enquêtes démographiques ou des enquêtes sur la population active. La catégorie des travailleurs indépendants est donc formée de la somme des employeurs et des travailleurs à leur compte. Le nombre de femmes (hommes) employeuses (employeurs) correspond au nombre de femmes (hommes) qui déclarent avoir le statut de " travailleur indépendant employant des salariés " lors des enquêtes démographiques. Le nombre de femmes (hommes) travaillant à leur compte correspond au nombre de femmes (hommes) qui déclarent avoir le statut de " travailleur indépendant sans salarié ". La proportion de femmes (hommes) employeuses ou travailleuses à leur compte est calculée par rapport au nombre total de femmes (hommes) ayant un emploi.

L'écart femmes-hommes en matière de taux de travail indépendant pour l'année $t$ correspond à la différence entre le taux de travail indépendant des hommes et celui des femmes pour l'année t. La variation du taux de travail indépendant des femmes (hommes) correspond à la différence entre le taux de travail indépendant des femmes (hommes) pour l'année t et ce taux pour l'année $\mathrm{t}-\mathrm{n}$.

La proportion de travailleuses indépendantes (travailleurs indépendants) dans la population des jeunes actives occupées (jeunes actifs occupés) est le produit de la division du nombre de travailleuses indépendantes (travailleurs indépendants) ayant entre 15 et 29 ans par le nombre total de femmes (hommes) occupées qui ont entre 15 et 29 ans.
La proportion de travailleuses indépendantes (travailleurs indépendants) dans la population des femmes (hommes) occupées de nationalité étrangère est le produit de la division du nombre de travailleuses indépendantes (travailleurs indépendants) de nationalité étrangère par le nombre total de femmes (hommes) occupées de nationalité étrangère

La proportion de femmes (hommes) salariées ayant une seconde activité qu'elles (ils) exercent comme travailleuses indépendantes (travailleurs indépendants) est le produit de la division du nombre de femmes (hommes) salariées déclarant avoir un deuxième emploi qu'elles (ils) exercent comme travailleuses indépendantes (travailleurs indépendants) par le nombre total de femmes (hommes) salariées.

Informations sur les données concernant Israël : $h t t p: / / d x$.doi. org/10.1787/888932315602.

\section{Comparabilité}

Le principal problème de comparabilité concerne la classification des " travailleurs indépendants " propriétaires d'entreprises constituées en sociétés. Certains pays, notamment le Japon, la NouvelleZélande et la Norvège, ne prennent en compte que les travailleurs indépendants propriétaires d'entreprises non constituées en sociétés, conformément au SCN 2008, ce qui peut se traduire, pour ces pays, par une distorsion vers le bas du nombre de travailleurs indépendants propriétaires d'entreprises ayant des salariés.

$\mathrm{Au}$ graphique 6.6, les activités de services recouvrent les secteurs 45-96 de la CITI Rév. 4. Au graphique 6.7, les données se rapportent aux travailleurs indépendants de nationalité étrangère pour tous les pays, à l'exception des États-Unis, pour lesquels les données portent sur les personnes nées à l'étranger.

Tous les travailleurs indépendants ne sont pas nécessairement entrepreneurs au sens strict du terme, tel qu'il est défini dans le Programme d'indicateurs de l'entrepreneuriat OCDE-Eurostat. Les statistiques du travail indépendant prennent en compte, par exemple, les artisans qui exercent leur activité à un faible niveau, souvent à titre récréatif. Il importe donc d'interpréter les données avec précaution lors des analyses relatives à l'entrepreneuriat.

\section{Sources}

Afrique du Sud : Labour Force Survey, http://www.statssa.gov. $\mathrm{za} /$ ?page_id=1854\&PPN=P0211.

Brésil : National Household Sample Survey, http://www.ibge.gov. br/english/estatistica/populacao/trabalhoerendimento/pnad2008/ default.shtm\#brasil.

Canada : Enquête sur la population active, http://www23.statcan. gc.ca/imdb/p2SV_f.pl?Function=getSurvey\&SDDS=3701.

Chili : Encuesta Nacional del Empleo, http://www.ine.cl/estadisticas/ laborales/ene.

États-Unis : Current Population Survey, www.census.gov/cps/.

Eurostat : Enquête de l'UE sur les forces de travail, http://ec.europa. eu/eurostat/web/microdata/european-union-labour-force-survey.

Israël : Labour Force Survey, http://www.cbs.gov.il/ts/databank/ databank_main_func_e.html? $i=21 \& t i=11 \& r=0 \& f=3 \& 0=0$.

Japon : Labour Force Survey, http://www.e-stat.go.jp/SG1/estat/ eStatTopPortalE.do.

Mexique : Encuesta National de Empleo, http://www.inegi.org.mx/ est/contenidos/proyectos/encuestas/hogares/default.aspx.

\section{Pour en savoir plus}

OCDE (2017), Rapport sur la mise en œuure des Recommandations de l'OCDE sur l'égalité hommes-femmes, Réunion du Conseil de l'OCDE au niveau des Ministres, Paris, 7-8 juin 2017, http:// www.oecd.org/fr/rcm/documents/C-MIN-2017-7-FR.pdf. 
6. ENTREPRENEURIAT FÉMININ

Taux de travail indépendant : différences entre femmes et hommes

Graphique 6.1. Évolution du taux de travail indépendant, moyenne des pays de l'OCDE, par catégorie et par sexe $2007=100$

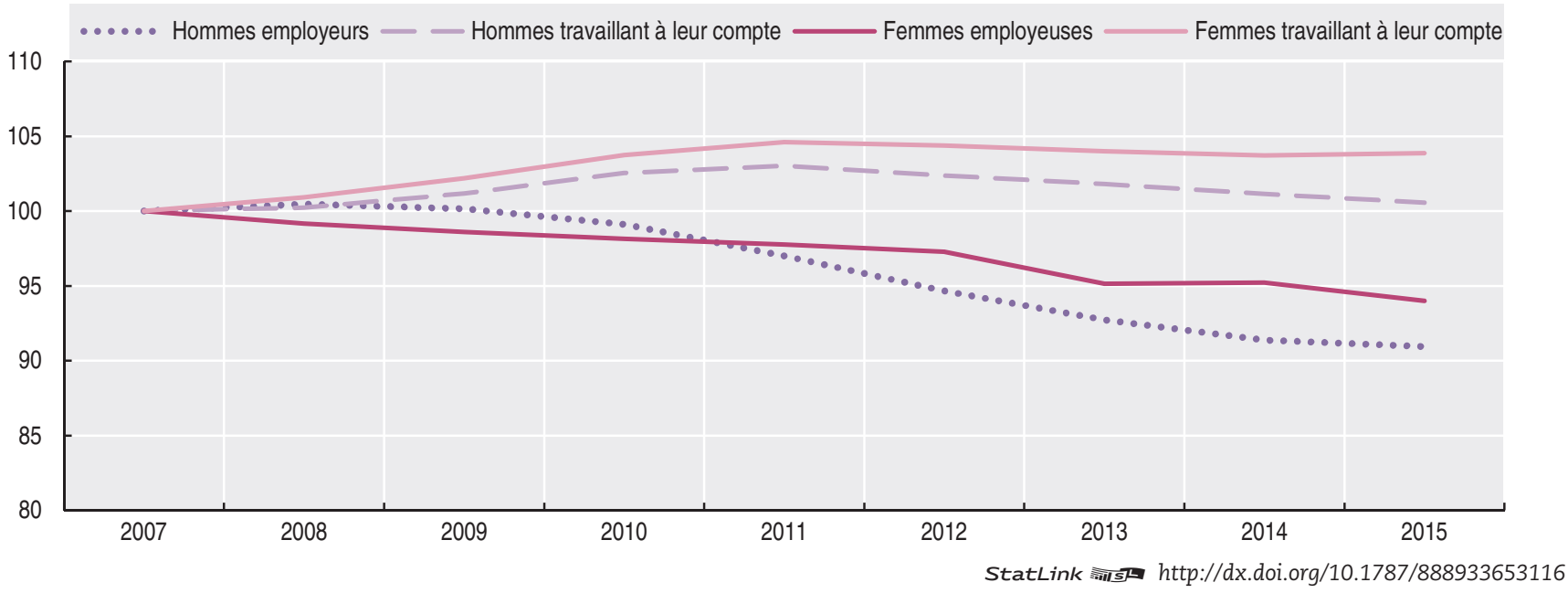

Tableau 6.1. Nombre de travailleurs indépendants par catégorie et par sexe

En milliers de personnes, 2016 ou dernière année disponible

\begin{tabular}{|c|c|c|c|c|c|}
\hline \multirow{2}{*}{ Pays } & \multirow{2}{*}{ Âge } & \multicolumn{2}{|c|}{ Employeurs } & \multicolumn{2}{|c|}{ Travailleurs à leur compte } \\
\hline & & Hommes & Femmes & Hommes & Femmes \\
\hline Australie & $15+$ & 494 & 236 & 818 & 431 \\
\hline Autriche & $15-64$ & 137 & 49 & 153 & 110 \\
\hline Belgique & $15-64$ & 137 & 49 & 284 & 144 \\
\hline Canada & $15-64$ & 559 & 220 & 1,001 & 729 \\
\hline Chili & $15+$ & 253 & 80 & 946 & 684 \\
\hline République tchèque & $15-64$ & 117 & 34 & 431 & 229 \\
\hline Danemark & $15-64$ & 67 & 23 & 80 & 40 \\
\hline Estonie & $15-64$ & 18 & 7 & 21 & 13 \\
\hline Finlande & $15-64$ & 71 & 22 & 130 & 73 \\
\hline France & $15-64$ & 807 & 285 & 1,132 & 666 \\
\hline Allemagne & $15-64$ & 1,246 & 436 & 1,237 & 824 \\
\hline Grèce & $15-64$ & 188 & 74 & 528 & 274 \\
\hline Hongrie & $15-64$ & 146 & 61 & 138 & 88 \\
\hline Islande & $15-64$ & 5 & 2 & 9 & 4 \\
\hline Irlande & $15-64$ & 66 & 21 & 156 & 43 \\
\hline Israël & $15+$ & 119 & 26 & 172 & 116 \\
\hline Italie & $15-64$ & 992 & 362 & 2,299 & 1,121 \\
\hline Japon & $15-64$ & 107 & 23 & 287 & 110 \\
\hline Corée & $15+$ & 1,203 & 378 & 2,771 & 1,211 \\
\hline Lettonie & $15-64$ & 25 & 10 & 38 & 29 \\
\hline Lituanie & $15-64$ & 22 & 9 & 71 & 46 \\
\hline Luxembourg & $15-64$ & 7 & 3 & 8 & 6 \\
\hline Mexique & $15-64$ & 1,536 & 408 & 5,948 & 3,829 \\
\hline Pays-Bas & $15-64$ & 243 & 86 & 572 & 377 \\
\hline Nouvelle-Zélande & $15-64$ & 25 & 13 & 66 & 44 \\
\hline Norvège & $15-64$ & 34 & 11 & 74 & 40 \\
\hline Pologne & $15-64$ & 429 & 179 & 1,471 & 731 \\
\hline Portugal & $15-64$ & 134 & 63 & 244 & 167 \\
\hline Roumanie & $15-64$ & 63 & 24 & 925 & 333 \\
\hline République slovaque & $15-64$ & 57 & 21 & 204 & 94 \\
\hline Slovénie & $15-64$ & 25 & 8 & 48 & 23 \\
\hline Afrique du Sud & $15-64$ & 631 & 152 & 786 & 626 \\
\hline Espagne & $15-64$ & 600 & 270 & 1,348 & 712 \\
\hline Suède & $15-64$ & 127 & 37 & 162 & 84 \\
\hline Suisse & $15-64$ & 179 & 66 & 126 & 126 \\
\hline Turquie & $15-64$ & 1,087 & 109 & 3,403 & 697 \\
\hline Royaume-Uni & $15-64$ & 505 & 188 & 2,376 & 1,227 \\
\hline Etats-Unis & $16-64$ & 2,408 & 766 & 6,026 & 3,798 \\
\hline
\end{tabular}




\section{ENTREPRENEURIAT FÉMININ}

Taux de travail indépendant : différences entre femmes et hommes

Graphique 6.2. Pourcentage de travailleurs indépendants, par catégorie et par sexe

Pourcentage de l'emploi total, 2016 ou dernière année disponible

Total

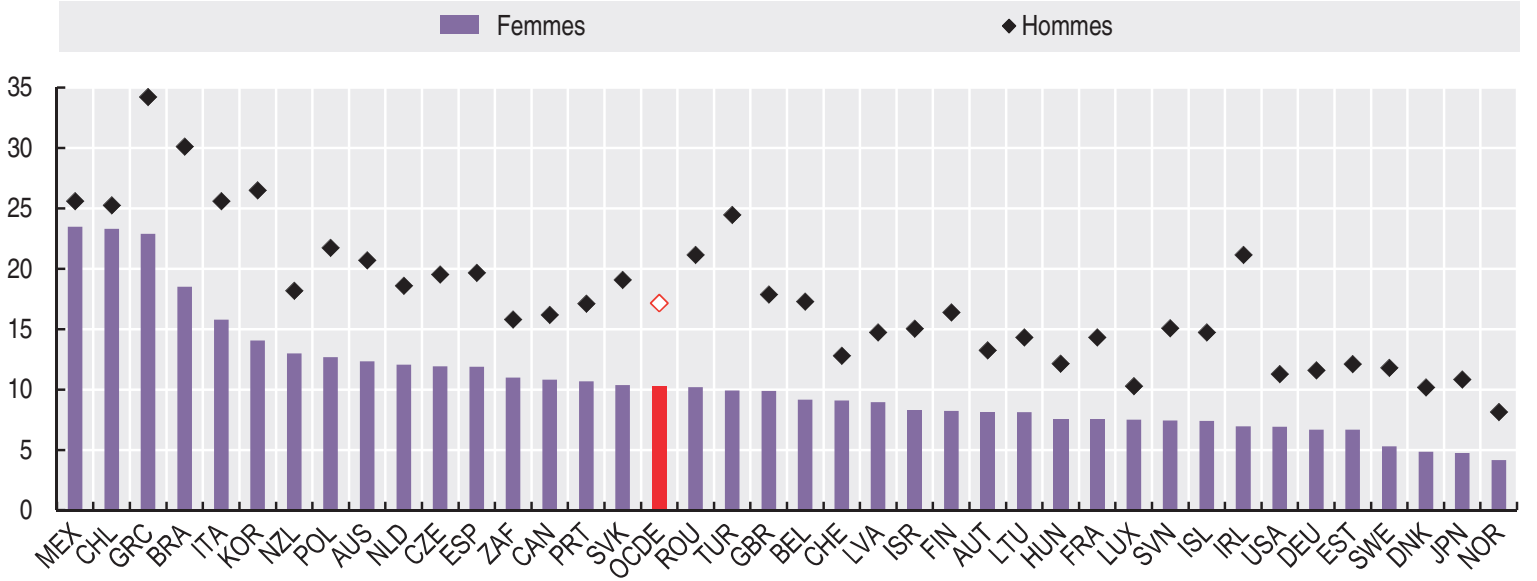

Employeurs

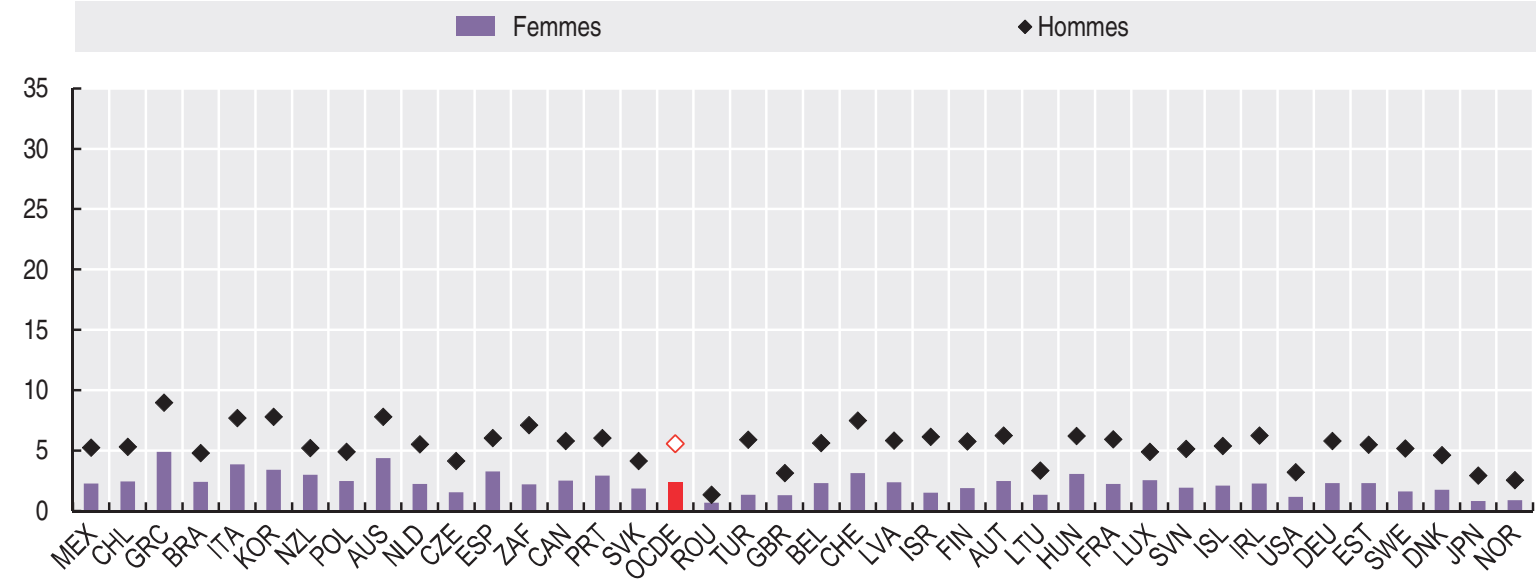

Travailleurs à leur compte

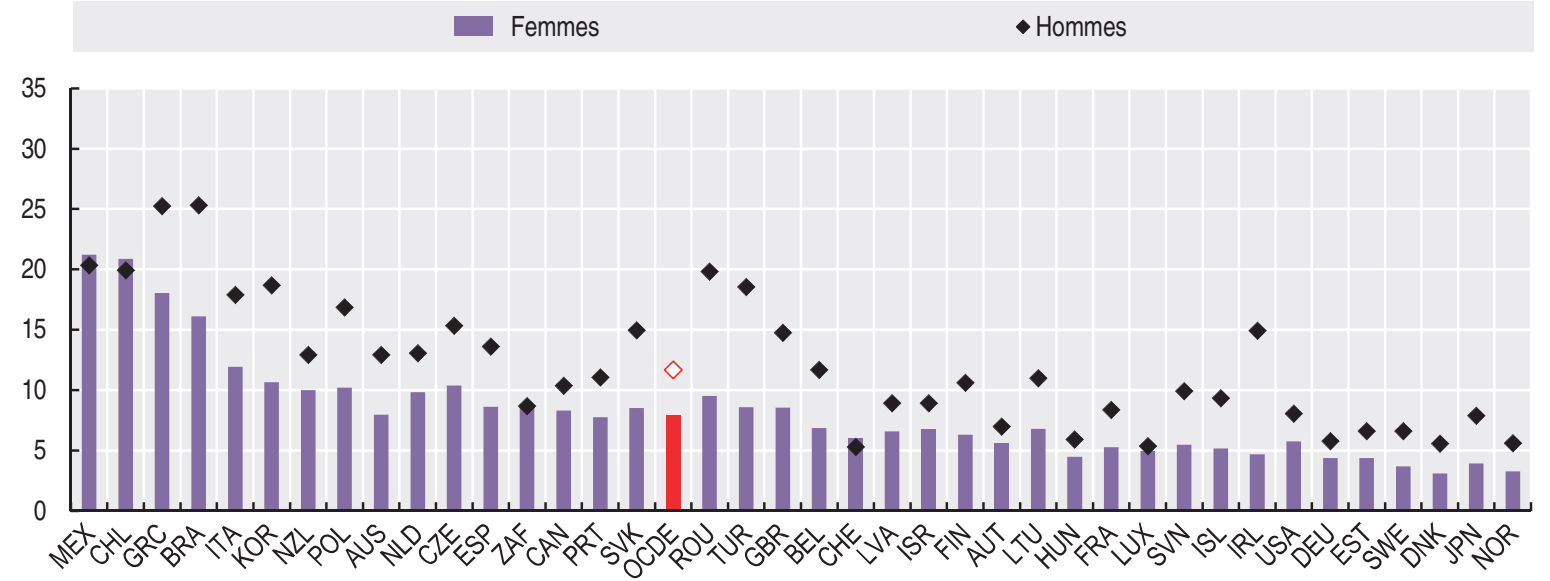

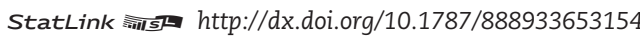


6. ENTREPRENEURIAT FÉMININ

Taux de travail indépendant : différences entre femmes et hommes

Graphique 6.3. Écart de taux de travail indépendant entre femmes et hommes En points de pourcentage

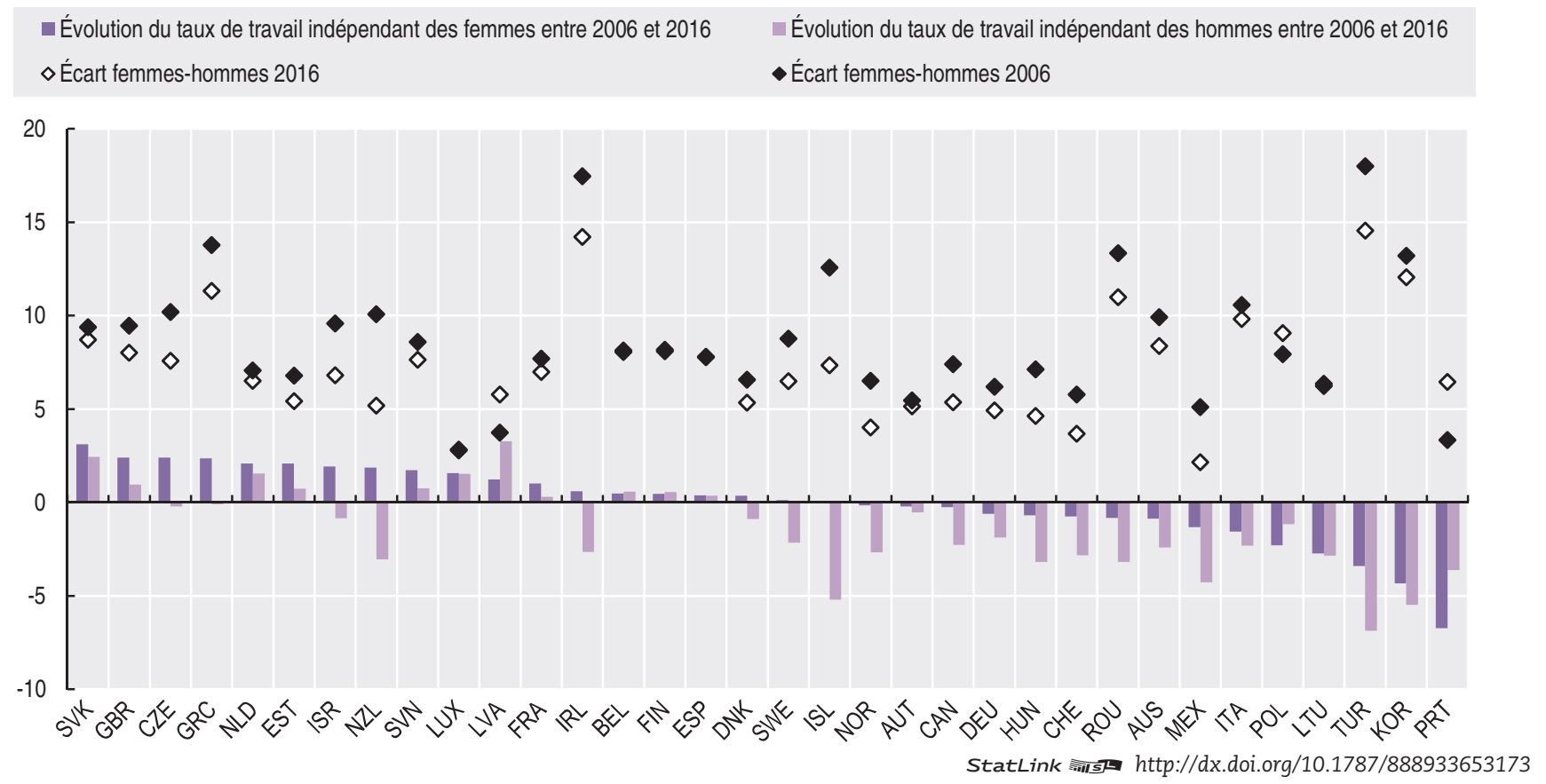

Graphique 6.4. Pourcentage de travailleurs indépendants dans la population des jeunes actifs occupés, par sexe En pourcentage de l'emploi total chez les personnes de moins de 30 ans, moyenne 2015-2016 ou dernière année disponible

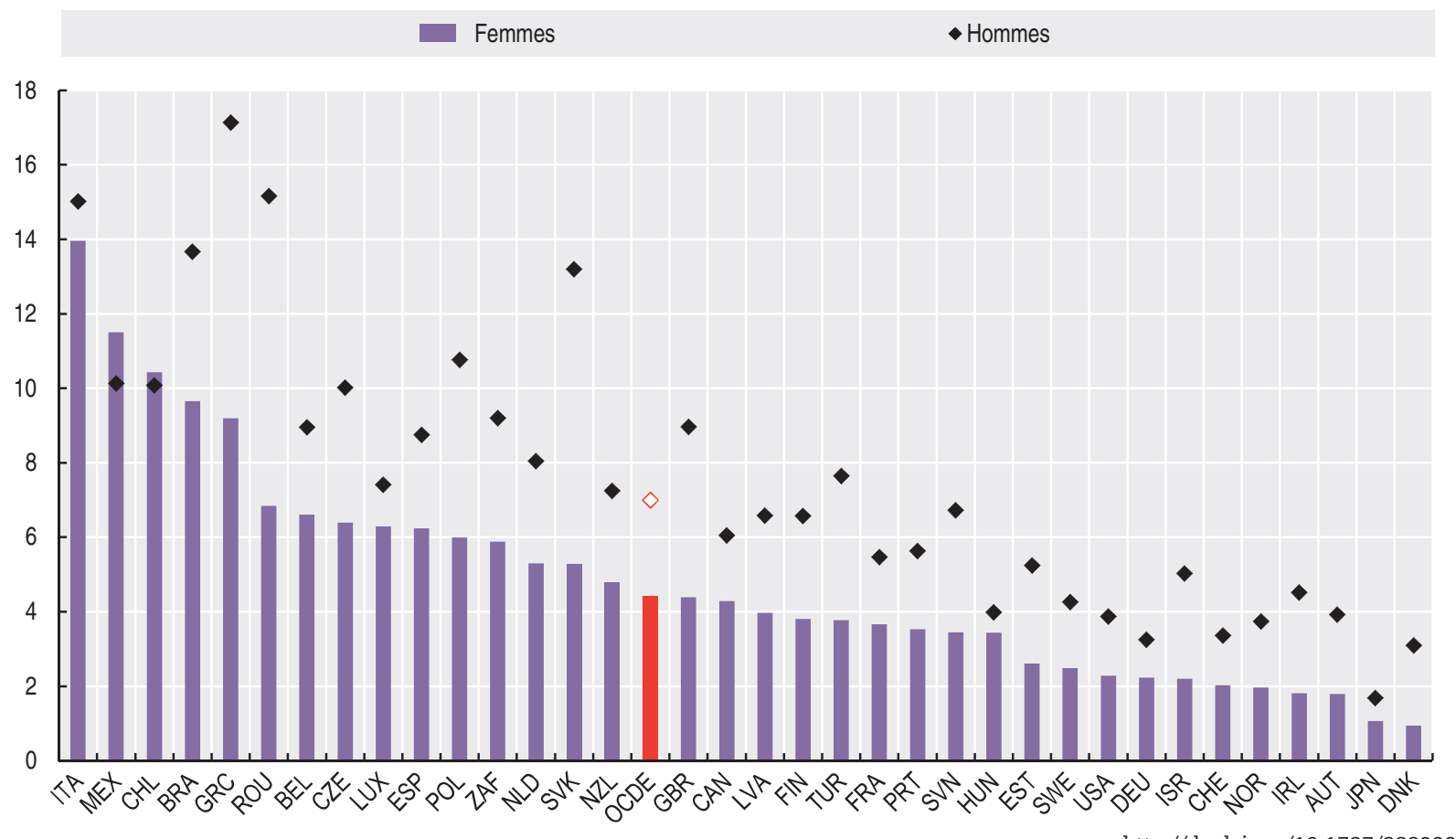

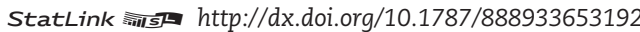




\section{ENTREPRENEURIAT FÉMININ}

Taux de travail indépendant : différences entre femmes et hommes

Graphique 6.5. Travailleurs indépendants exerçant dans les activités de fabrication et la construction

En pourcentage du nombre total de travailleurs indépendants par sexe, 2016 ou dernière année disponible

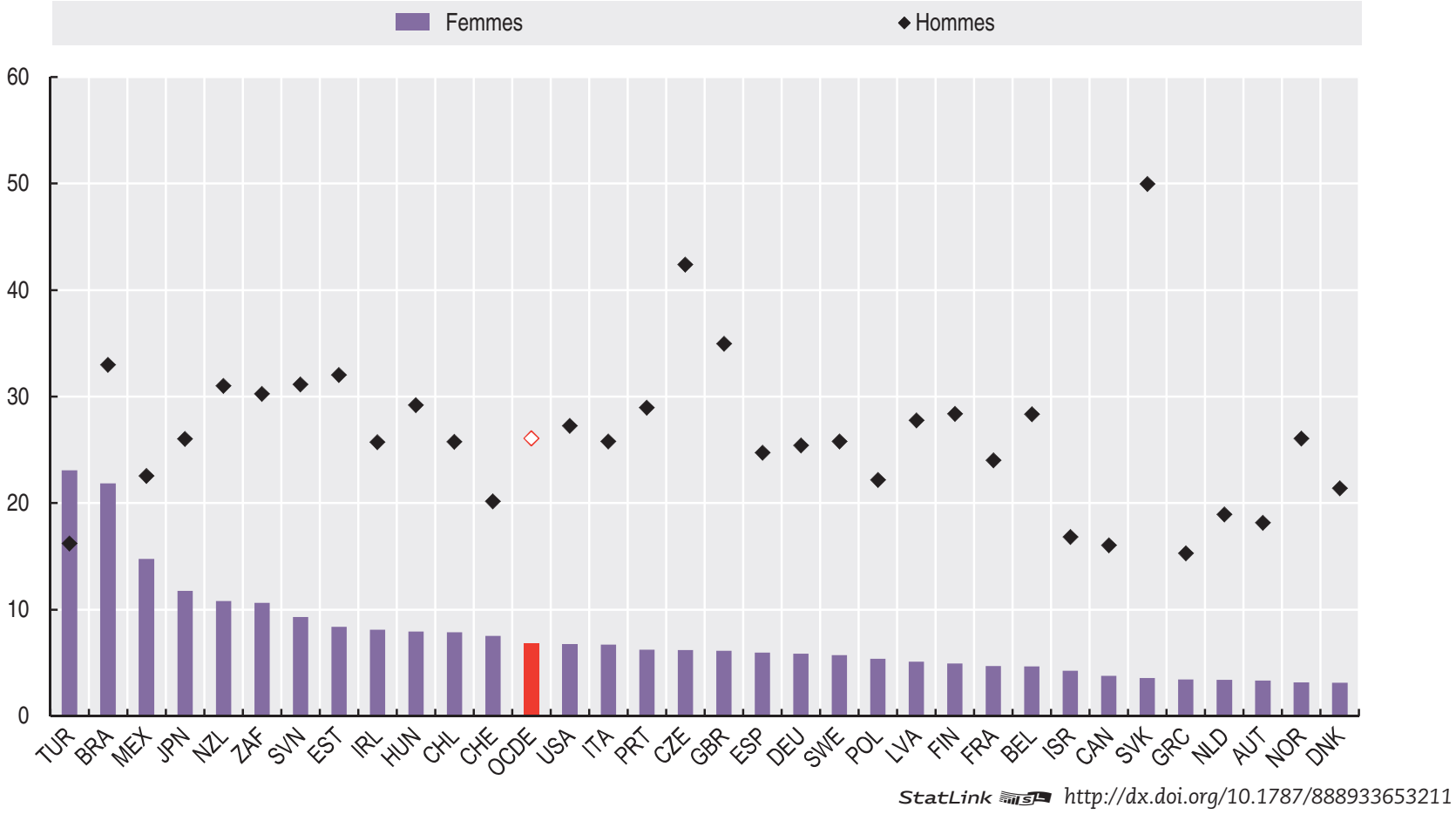

Graphique 6.6. Travailleurs indépendants exerçant dans les services

En pourcentage du nombre total de travailleurs indépendants par sexe, 2016 ou dernière année disponible

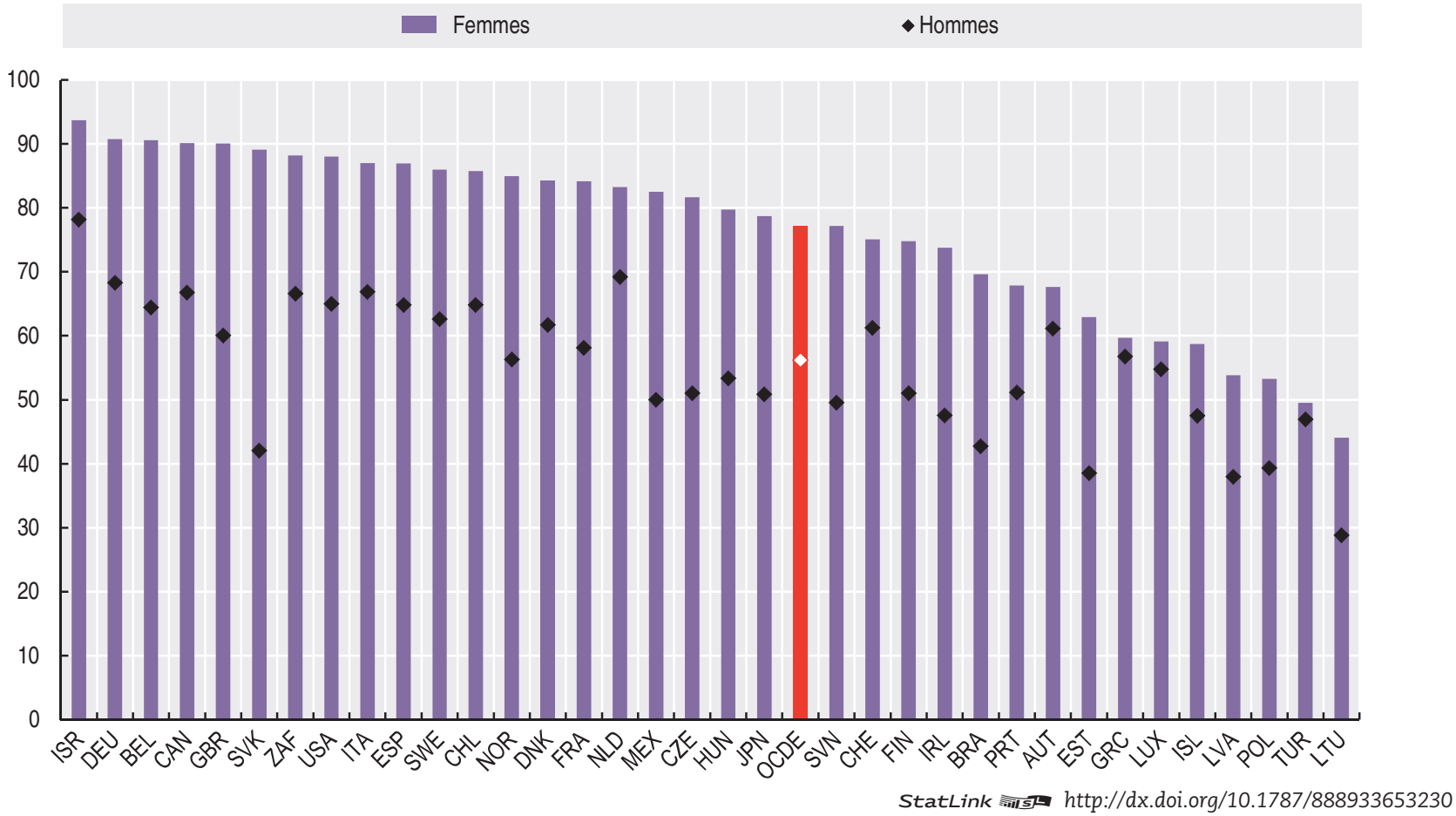


6. ENTREPRENEURIAT FÉMININ

Taux de travail indépendant : différences entre femmes et hommes

Graphique 6.7. Pourcentage de travailleurs indépendants dans la population des personnes occupées de nationalité étrangère

En pourcentage de l'emploi total chez les personnes de nationalité étrangère, 2016 ou dernière année disponible

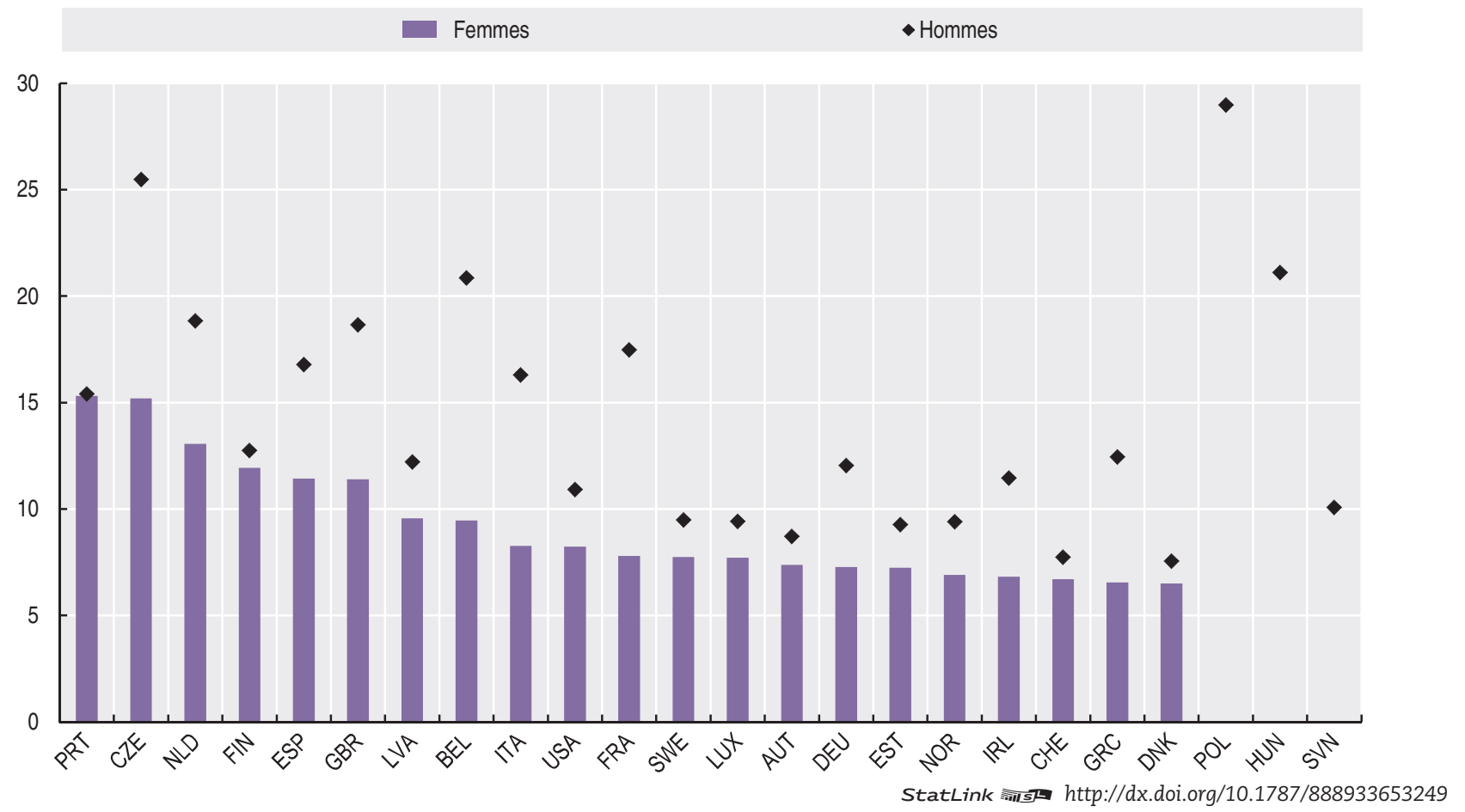

Graphique 6.8. Pourcentage de salariés exerçant comme travailleurs indépendants à titre de deuxième emploi, par sexe

Pourcentage de l'ensemble des salariés, par sexe

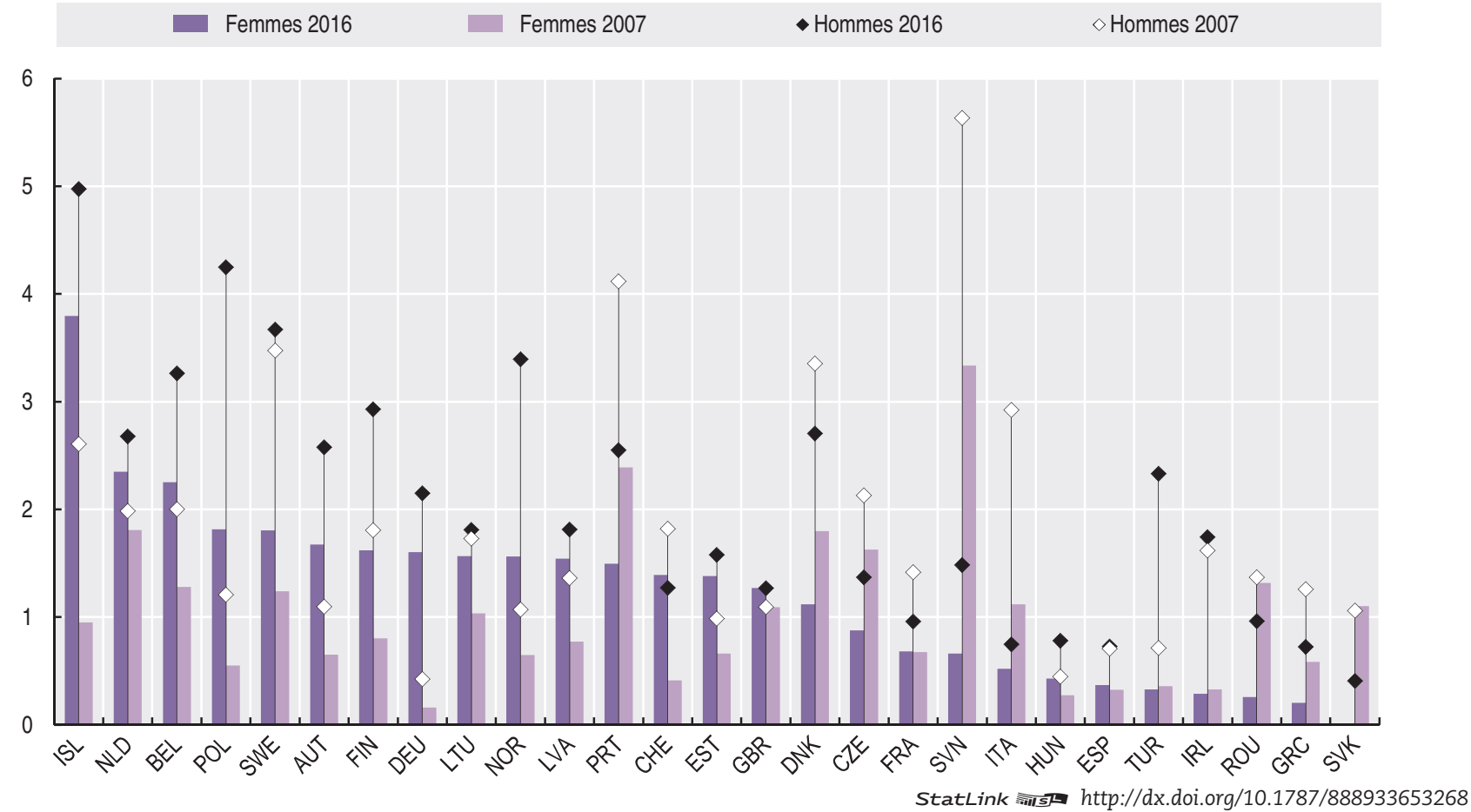




\section{À savoir}

- En 2014, les femmes travailleuses indépendantes gagnaient $10 \%$ de moins que les hommes au Luxembourg et en Lituanie, mais presque $60 \%$ de moins qu'eux en Pologne, aux États-Unis et en Roumanie. Au cours de la période 2007-14, l'écart femmes-hommes en matière de revenus du travail indépendant a diminué dans la majorité des pays, à l'exception de la Pologne, de l'Italie, de la République slovaque, de la Slovénie et de la Roumanie.

- Parmi les facteurs qui peuvent expliquer l'écart entre femmes et hommes en matière de revenus du travail indépendant, figure le nombre hebdomadaire moyen d'heures d'activité des travailleurs indépendants : il est généralement plus élevé chez les hommes que chez les femmes dans l'ensemble des pays. Globalement, les travailleurs indépendants tendent à effectuer en moyenne davantage d'heures que les salariés ; toutefois, dans quelques pays, comme la Lettonie, la Turquie ou le Royaume-Uni, les travailleuses indépendantes ont en moyenne un temps hebdomadaire d'activité plus court que les salariés masculins et féminins.

\section{Pertinence}

La crainte de percevoir des revenus faibles ou irréguliers est une des principales raisons qui dissuadent beaucoup de personnes de s'engager dans l'entrepreneuriat. Si ce dernier est un moyen de s'enrichir pour ceux qui réussissent, beaucoup de travailleurs indépendants doivent survivre avec des revenus relativement faibles et finissent par effectuer des journées plus longues que les salariés. Or, qui dit faibles revenus dit moindre possibilité d'épargner et donc risque plus grand de tomber dans la pauvreté en cas d'échec de l'entreprise.

\section{Définitions}

L'écart femmes-hommes en matière de revenus du travail indépendant correspond à la différence de revenus moyens du travail indépendant entre les femmes et les hommes, divisée par les revenus moyens du travail indépendant des hommes. Les revenus tiennent compte ici des pertes éventuelles. La variation de l'écart femmes-hommes en matière de revenus du travail indépendant est la différence d'écart de revenus du travail indépendant entre les sexes d'une année à l'autre, en points de pourcentage.

Le nombre moyen d'heures de travail correspond au nombre hebdomadaire d'heures de travail qu'une personne occupée effectue normalement. Il comprend toutes les heures travaillées, y compris les heures supplémentaires, qu'elles soient rémunérées ou non. Il ne tient pas compte du temps de trajet entre le domicile et le lieu de travail, ni de la pause du repas principal (prise normalement à midi).

\section{Comparabilité}

Des problèmes d'ordre méthodologique compliquent la comparaison des statistiques relatives aux revenus d'un pays et d'une période à l'autre. Les travailleurs indépendants ont souvent des pratiques comptables qui leur permettent difficilement d'apporter des réponses précises aux questions posées sur leurs revenus lors des enquêtes. De plus, leur cadre financier et comptable ne correspond pas bien à celui qui sert à l'élaboration des comptes nationaux ou à l'analyse du revenu des ménages. Il est en outre important de prendre en compte l'écart entre femmes et hommes quant au nombre d'heures d'activité des travailleurs indépendants.

Les femmes consacrent généralement davantage de temps que les hommes au travail familial et domestique non rémunéré ; c'est une donnée dont il faut tenir compte lors de l'examen du nombre moyen d'heures d'activité des travailleurs indépendants.

\section{Sources/bases de données en ligne}

Canada : Enquête sur la dynamique du travail et du revenu \& Enquête canadienne sur le revenu.

États-Unis : Current Population Survey (CPS), American Community Survey (ACS), Survey of Income and Program Participation (SIPP).

Europe : Enquêtes sur les forces de travail, Statistiques de l'Union européenne sur le revenu et les conditions de vie (EU-SILC) et Enquête de l'UE sur les forces de travail.

Mexique : Encuesta Nacional de Ocupación y Empleo.

Nouvelle-Zélande : Income Survey et Labour Force Survey.

\section{Pour en savoir plus}

Hamilton, B. H. (2000). « Does Entrepreneurship Pay? An Empirical Analysis of the Returns to Self-Employment ", Journal of Political Economy, University of Chicago Press, vol. 108, $n^{\circ} 3$, pages 604-631, juin.

OCDE (2017), Rapport sur la mise en œuure des Recommandations de l'OCDE sur l'égalité hommes-femmes, Réunion du Conseil de l'OCDE au niveau des Ministres, Paris, 7-8 juin 2017, http:// www.oecd.org/fr/rcm/documents/C-MIN-2017-7-FR.pdf.

OCDE (2014), Enhancing Women's Economic Empowerment through Entrepreneurship and Business Leadership in OECD Countries, Background Report, http://www.oecd.org/gender/Enhancing\%20 Women\%20Economic\%20Empowerment_Fin_1_Oct_2014.pdf.

OCDE (2012), Inégalités hommes-femmes : il est temps d'agir, Éditions OCDE, Paris, http://dx.doi.org/10.1787/9789264179660-fr.

OCDE/Union européenne (2015), Pallier la pénurie d'entrepreneurs 2015 : Politiques en faveur du travail indépendant et de l'entrepreneuriat, Éditions OCDE, Paris, http://dx.doi.org/ 10.1787/9789264249936-fr. 
6. ENTREPRENEURIAT FÉMININ

Revenus du travail indépendant

Graphique 6.9. Écart de revenus du travail indépendant entre femmes et hommes Différence de revenus entre les hommes et les femmes en pourcentage des revenus des hommes

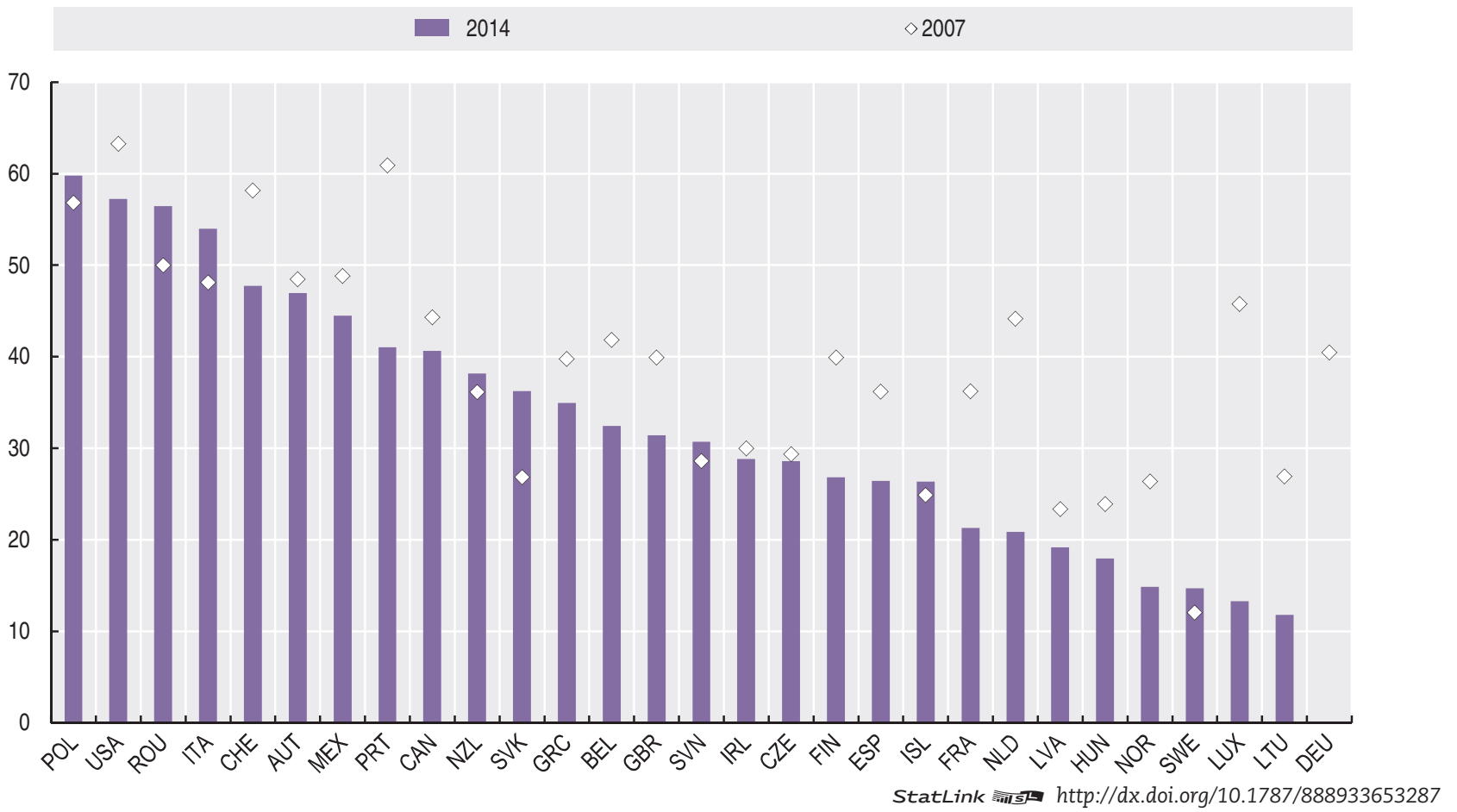

Graphique 6.10. Nombre moyen d'heures de travail selon le statut professionnel et le sexe

Nombre hebdomadaire moyen d'heures de travail effectuées dans l'emploi principal, 2016 ou dernière année disponible

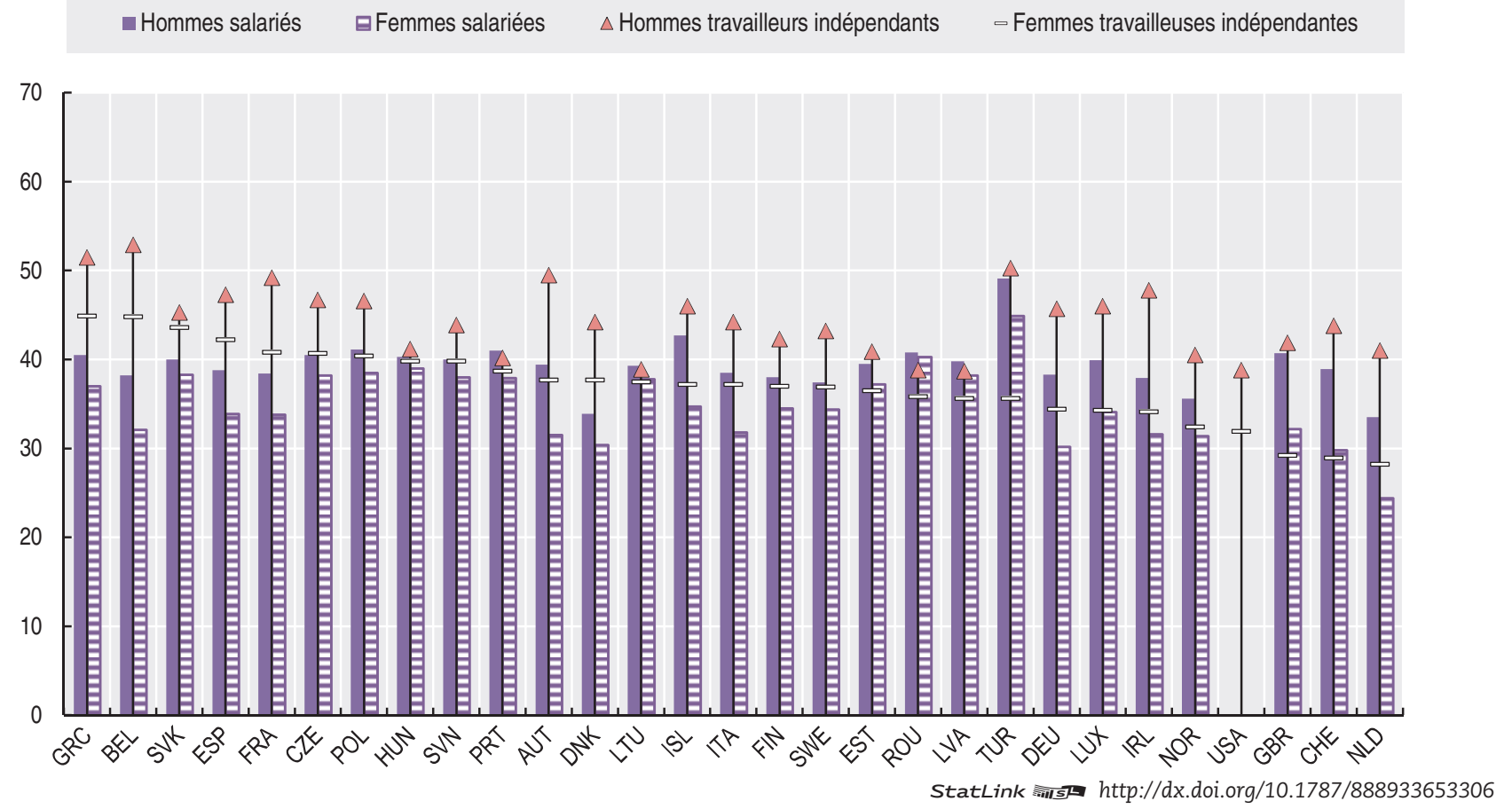




\section{À savoir}

- L'analyse des faits observés montre généralement qu'il existe une différence entre les femmes et les hommes dans la perception des obstacles à la création d'entreprises. Cependant, les femmes se sentent tout aussi confiantes que les hommes au sujet de leur entreprise et de son avenir, notamment des perspectives de création d'emplois, une fois que celle-ci est opérationnelle.

- La formation et les médias en ligne sont considérés aussi bien par les femmes que par les hommes comme des sources de connaissances utiles pour améliorer leur aptitude à gérer une entreprise. En revanche, les femmes dirigeantes d'entreprise tendent davantage que leurs homologues masculins à mettre à profit l'expérience des membres de leur famille ou d'amis, alors que les hommes chefs d'entreprise préfèrent apprendre auprès de leurs pairs.

- Les entreprises dirigées par des hommes sont plus souvent engagées dans le commerce international, pour faire de l'exportation ou de l'importation, que celles qui ont une femme à leur tête. De plus, une part appréciable des entreprises dirigées par des hommes (en moyenne $33 \%$ dans les pays de l'OCDE) exportent uniquement vers des entreprises, alors que dans tous les pays, une forte proportion des entreprises dirigées par des femmes exportent seulement vers des consommateurs (50\% en moyenne dans les pays de l'OCDE), ce qui tient en partie aux différences existant quant aux secteurs d'activité où les femmes et les hommes chefs d'entreprise sont respectivement le plus présents.

\section{Pertinence}

L'attitude des individus à l'égard du risque entrepreneurial, ainsi que la confiance qu'ils éprouvent en tant que propriétaires d'entreprises résultent de la conjonction de diverses caractéristiques personnelles (dont les compétences acquises au cours de la formation et de la vie professionnelle) et de facteurs étroitement liés aux valeurs sociétales, ainsi que des conditions fondamentales de l'activité des entreprises. Les indicateurs qui permettent de mesurer les différences entre femmes et hommes quant à l'attitude à l'égard de l'entreprise peuvent apporter des informations précieuses pour étayer l'élaboration de politiques visant à promouvoir l'égalité entre les sexes dans le domaine de l'entrepreneuriat.

\section{Définitions}

Les entreprises appartenant à des hommes (femmes) ou gérées par des hommes (femmes) sont définies comme des entreprises dont au moins $65 \%$ des propriétaires ou des hauts responsables sont des hommes (femmes).

Une appréciation positive de la situation actuelle de l'entreprise et une appréciation positive des perspectives de l'entreprise correspondent à la réponse "Positive(s) " donnée respectivement aux questions suivantes : "Comment évaluez-vous la situation actuelle de votre entreprise ? " et "Quelles sont, selon vous, les perspectives de votre entreprise pour les six prochains mois ? ", les réponses possibles étant « Positive(s) ", " Neutre(s) » et " Négative(s) ». Les perspectives de création d'emplois à court terme sont mesurées d'après les perspectives d'évolution de l'emploi dans les entreprises, telles qu'elles ressortent de la réponse à la question "Comment prévoyez-vous l'évolution du nombre de salariés de votre entreprise au cours des six prochains mois ? ", réponse qui peut être l'une des suivantes : "Augmentation ", "Pas de changement » et " Diminution ».
Les sources de l'apprentissage sont déterminées d'après la proportion de répondants pour chacune des catégories de sources proposées dans la question à choix multiples suivante : "Quels moyens utilisez-vous pour acquérir de nouvelles connaissances utiles à la gestion de votre entreprise ? ", ces catégories de sources étant " Autres entreprises ", " Amis et famille ", " Recherches en ligne ", "Blogs et forums en ligne ", "Formation en ligne", "Formation en mode présentiel ", " Médias ".

L'engagement dans le commerce international est déterminé d'après les réponses positives à la question « Votre entreprise est-elle engagée dans le commerce international ? ", cette activité pouvant consister à faire uniquement de l'exportation, de l'importation, ou l'une et l'autre à la fois.

Les destinataires des exportations sont déterminés d'après la proportion de répondants pour chacune des catégories de destinataires entre lesquelles il est proposé de choisir, à savoir " Entreprises seulement ", " Consommateurs seulement » et « Les deux ».

Informations sur les données concernant Israël : http:// dx.doi.org/10.1787/888932315602.

\section{Comparabilité}

Les données sont tirées de l'enquête sur l'avenir des entreprises (Future of Business Survey), effectuée chaque mois par Facebook et conçue en collaboration avec la Direction des statistiques de l'OCDE et la Banque mondiale. Cette enquête est réalisée au moyen d'un questionnaire en ligne portant sur la perception qu'ont les personnes interrogées de la situation actuelle et des perspectives futures de leur entreprise et, plus généralement, de l'économie et du secteur d'activité concerné, ainsi que de l'évolution passée et attendue de l'emploi dans cette entreprise. L'enquête couvre à l'heure actuelle 42 pays développés ou émergents, la population de référence étant celle des entreprises ayant un compte Facebook. Les échantillons retenus pour chaque pays ne sont pas stratifiés et les graphiques figurant dans cette section présentent des données non pondérées selon la taille des entreprises, leur âge et leur activité économique.

Il y a lieu de faire preuve de circonspection lors de la comparaison des résultats de l'enquête concernant les pays développés avec ceux des pays en développement. Il a été délibérément choisi de faire porter cette enquête uniquement sur les entreprises ayant une présence sur Facebook. Dans les pays avancés, cette cohorte d'entreprises est susceptible d'être plus représentative de l'ensemble de la population des entreprises que dans les pays en développement.

\section{Source}

Enquête de Facebook sur l'avenir des entreprises (Future of Business Survey), www.futureofbusinesssurvey.org

\section{Pour en savoir plus}

Banque mondiale, Facebook, OCDE (2017), Future of Business Survey - Gender Management in Business, janvier 2017, www. futureofbusinesssurvey.org

Banque mondiale, Facebook, OCDE (2016), Future of Business Survey, septembre 2016, www.futureofbusinesssurvey.org

OCDE (2003), Business Tendency Surveys. A Handbook, Éditions OCDE, Paris, https://www.oecd.org/std/leadingindicators/31837055.pdf. 
Graphique 6.11. Appréciation positive de la situation actuelle et des perspectives de l'entreprise, selon le sexe des propriétaires ou des hauts responsables

En pourcentage des répondants à l'enquête, moyenne 2016-17
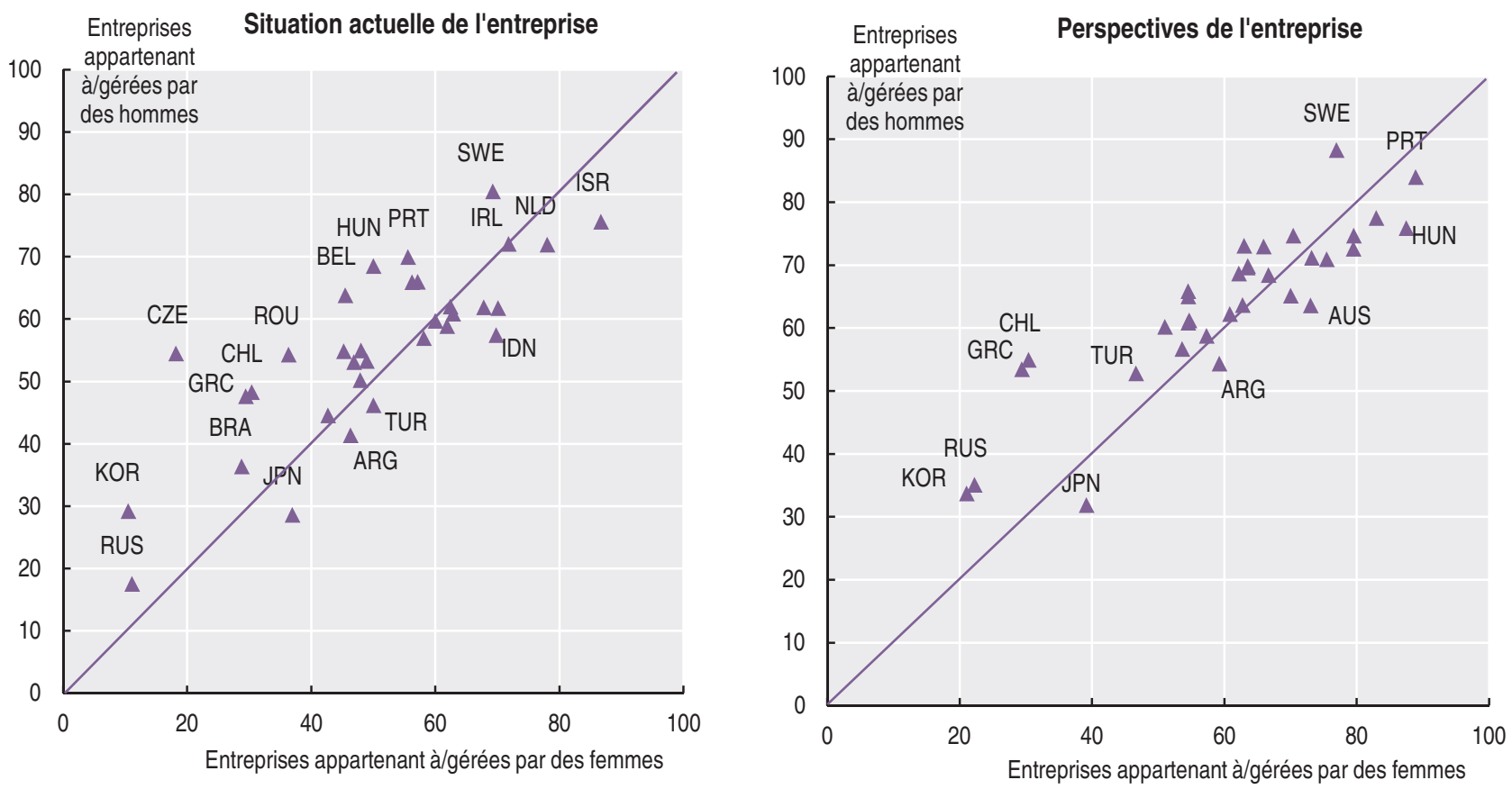

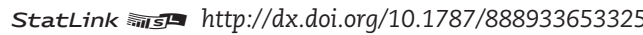

Graphique 6.12. Appréciation positive des perspectives de création d'emplois, selon le sexe des propriétaires ou des hauts responsables de l'entreprise

En pourcentage des répondants à l'enquête, moyenne 2016-17

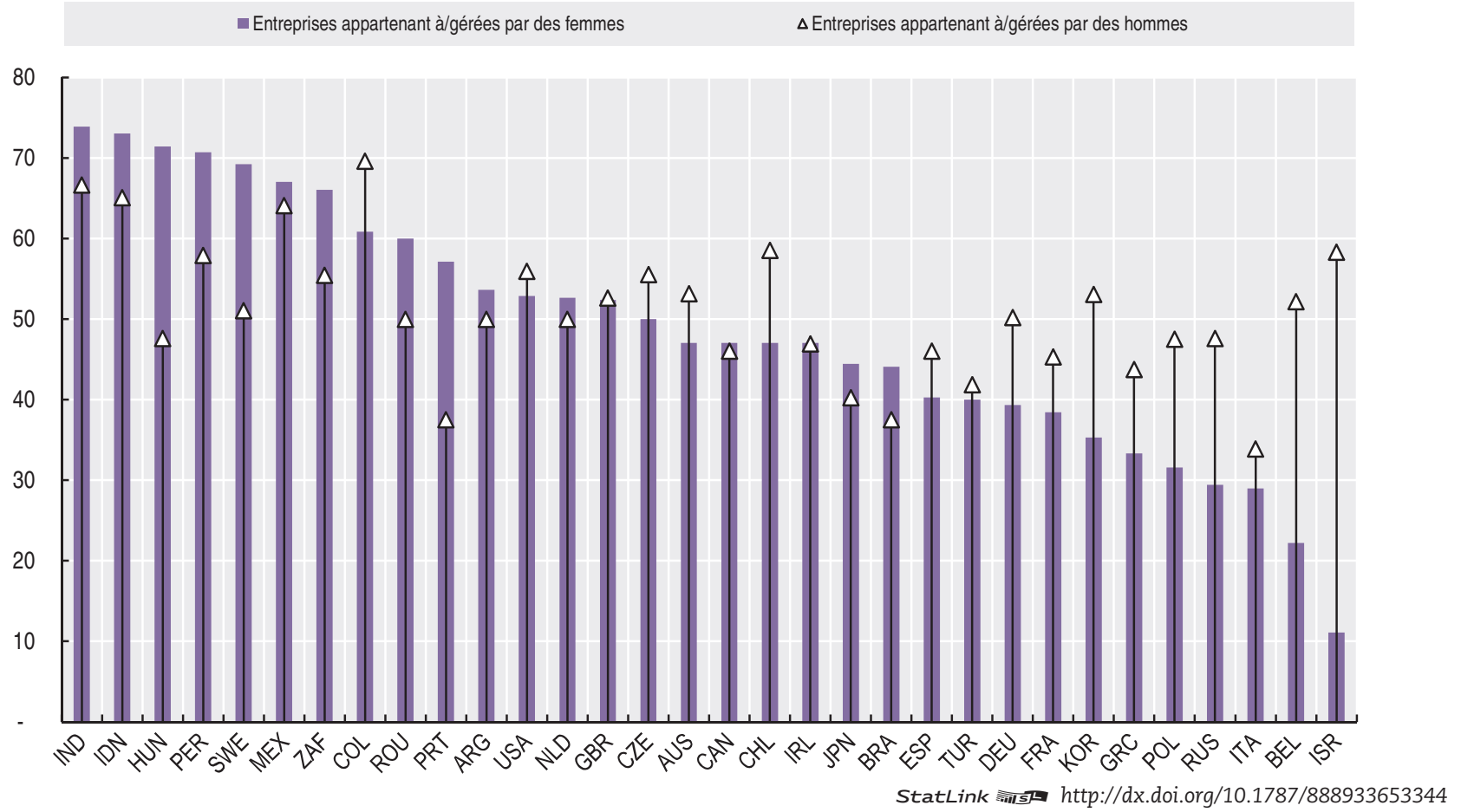




\section{ENTREPRENEURIAT FÉMININ}

\section{Attitude à l'égard de l'entrepreneuriat}

Graphique 6.13. Sources de l'apprentissage, selon le sexe des propriétaires ou des hauts responsables de l'entreprise En pourcentage des répondants à l'enquête, moyenne 2016-17
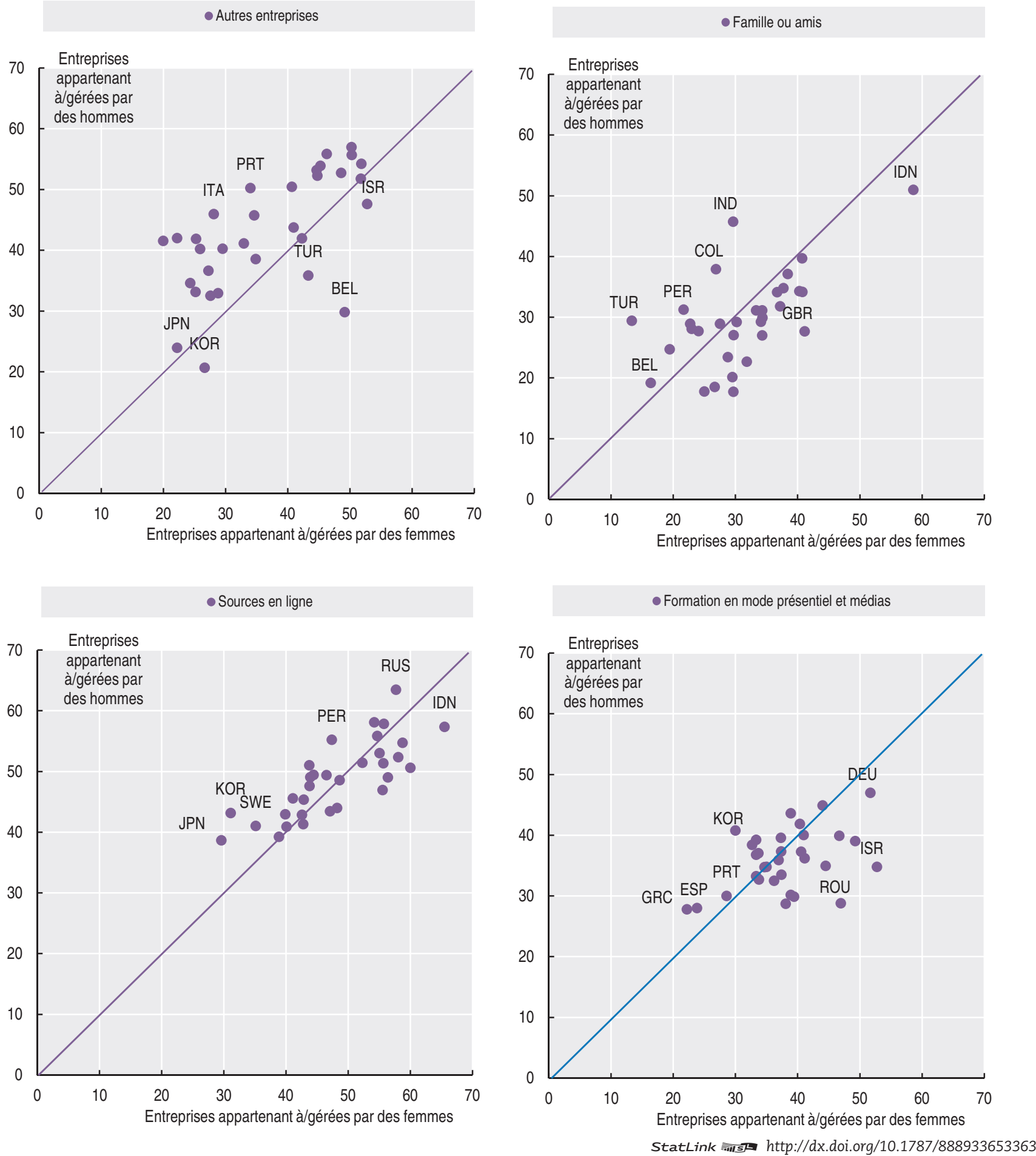
Graphique 6.14. Engagement dans le commerce international, selon le sexe des propriétaires ou des hauts responsables de l'entreprise

En pourcentage des répondants à l'enquête, moyenne 2016-17

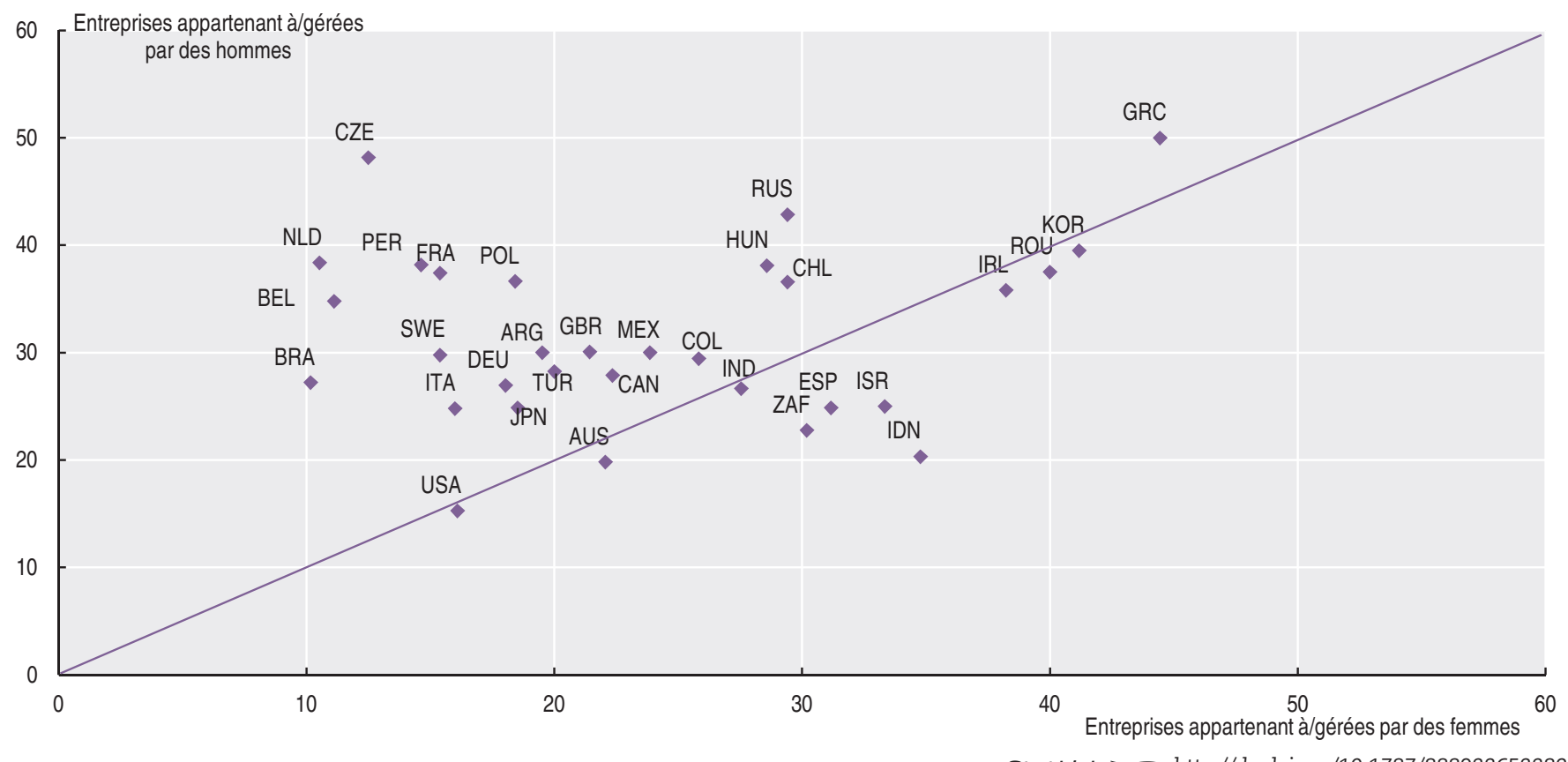

Graphique 6.15. Destinataires des exportations, selon le sexe des propriétaires ou des hauts responsables de l'entreprise

En pourcentage de l'ensemble des entreprises exportatrices, moyenne 2016-17
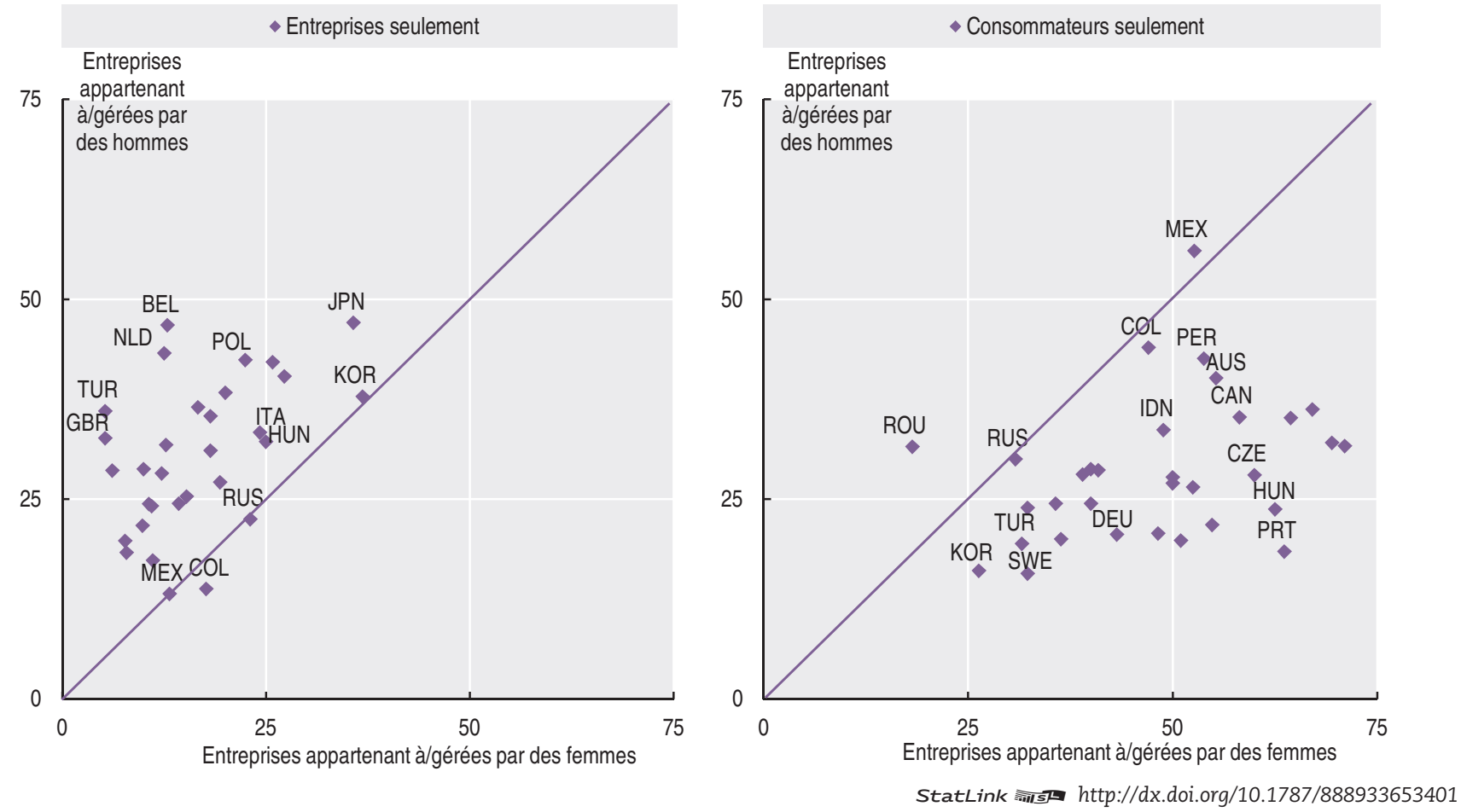
4.463 .72
228.72
285

309281.89

in 300.89 ? 40

$$
\beta
$$

$$
\begin{aligned}
& x^{3}+4^{2}, 05
\end{aligned}
$$

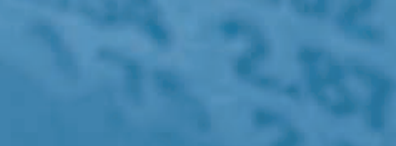
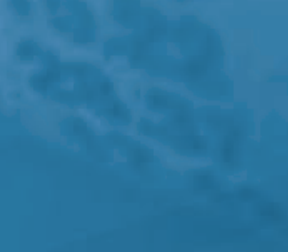

the

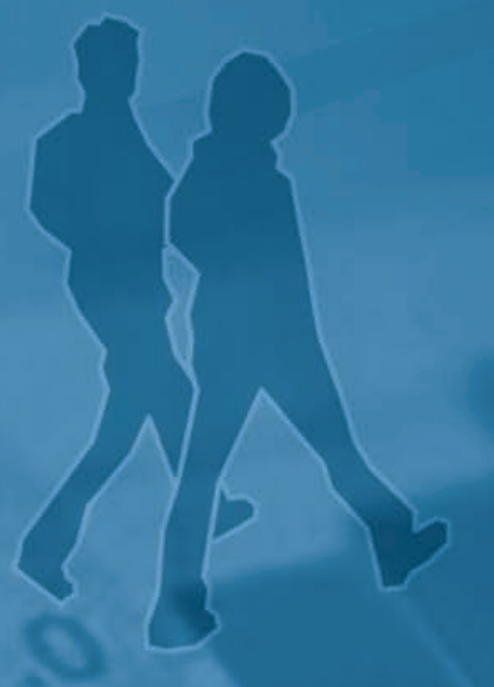

$-6 \times 2$

Q. 35

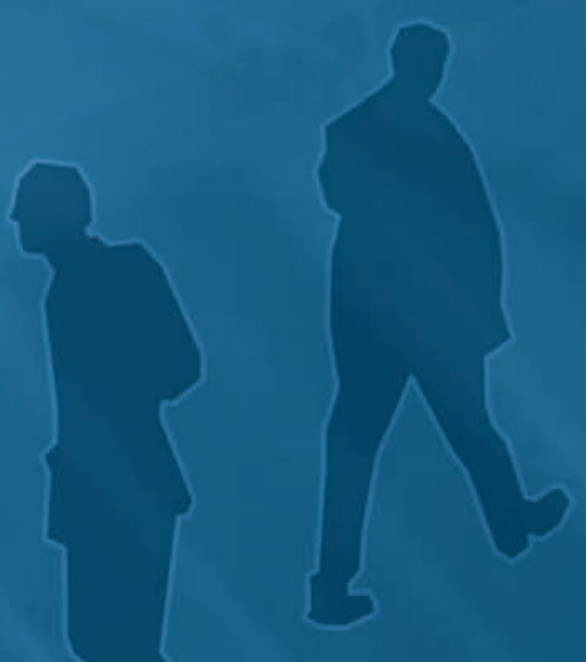




\section{CAPITAL-RISQUE}

Investissements de capital-risque

Investissements de capital-risque par entreprise bénéficiaire Investissements de capital-risque par secteur 


\section{À savoir}

- En 2016, les investissements de capital-risque aux États-Unis se sont élevés à 66.6 milliards USD et ont représenté $86 \%$ du total des investissements de ce type réalisés dans la zone OCDE. En Europe, ces investissements ont atteint 4.7 milliards USD.

- Dans la majorité des pays, le capital-risque représente une très petite part du PIB, souvent inférieure à $0.05 \%$. Les deux exceptions notables sont Israël et les États-Unis, où le secteur du capital-risque atteint un niveau de maturité supérieur et représente plus de $0.35 \%$ du PIB.

- De 2010 à 2016, l'évolution des investissements de capitalrisque a été variable selon les pays : aux États-Unis, en Corée, en Irlande et en Pologne, ces investissements ont plus que doublé. En revanche, en Australie, en Fédération de Russie, en Norvège et au Portugal, le niveau des investissements de capital-risque a été, en 2016, de moitié inférieur à ce qu'il était en 2010.

\section{Pertinence}

Le capital-risque est une forme de capital-investissement particulièrement intéressante pour les jeunes entreprises présentant un potentiel d'innovation et de croissance, mais dont le modèle économique n'a pas encore fait ses preuves et qui n'ont pas d'historique d'activité ; il remplace ou complète les financements bancaires classiques. Le développement de cette forme de financement est considéré comme une condition-cadre importante pour stimuler l'entrepreneuriat innovant.

\section{Définitions}

Le capital-risque est un sous-ensemble du capitalinvestissement (c'est-à-dire des fonds propres fournis à des entreprises non cotées en bourse) et désigne les investissements en fonds propres réalisés pour financer les phases de prédémarrage, de démarrage et de développement initial d'une entreprise (Source : Invest Europe).

Informations sur les données concernant Israël : http:// dx.doi.org/10.1787/888932315602.

\section{Comparabilité}

Il n'existe pas de définition internationale standard du capital-risque ou de la ventilation des investissements correspondants en fonction du stade de développement. De surcroît, la méthode de collecte des données diffère selon les pays.

Les données relatives au capital-risque proviennent principalement d'associations nationales ou régionales d'investisseurs de capital-risque qui les produisent ellesmêmes, dans certains cas avec le soutien de fournisseurs de données commerciales, à l'exception de l'Australie, où les statistiques sur le capital-risque sont recueillies et publiées par l'office statistique national australien.

Les statistiques présentées correspondent à l'agrégation de données sur les investissements en fonction de la localisation des sociétés de portefeuille, quelle que soit celle des sociétés de capital-investissement, sauf pour l'Australie, la Corée et le Japon, où les données se rapportent au lieu d'implantation des sociétés de capital-risque.

Pour Israël, les données concernent uniquement les entreprises financées par du capital-risque qui appartiennent au secteur de la haute technologie. Les données relatives aux ÉtatsUnis englobent aussi les investissements de capital-risque réalisés par des entreprises qui ne sont pas des sociétés de capital-risque à l'exclusion des investissements financés à $100 \%$ par des entreprises et/ou des business angels. Celles relatives à l'Australie, au Japon et à la Nouvelle-Zélande font référence à l'année budgétaire. Pour l'Europe, elles incluent seulement les investissements de capital-risque (amorçage, démarrage et stade ultérieur de développement) réalisés par des gestionnaires de fond conventionnels tels que les fonds de capital-investissement réalisant des investissements directs, les fonds mezzanine de capitalinvestissement, les fonds de coinvestissement ou les fonds de sauvetage/de retournement ; les investissements réalisés par des business angels, des incubateurs, des fonds d'infrastructure, des fonds immobiliers, des fonds de créances sinistrées, des fonds de fonds primaires ou les fonds de fonds secondaires sont exclus de ces données; le montant des investissements ne rend compte que du montant des capitaux propres investis par les gestionnaires de fond conventionnels et non de la valeur du cycle de financement dans son ensemble. Le capital-développement ou le capital destiné à financer le rachat d'entreprises par leurs cadres ou leurs salariés, actuellement ou auparavant financées à l'aide de capital-risque, ne sont pas non plus inclus.

Dans la Base de données de l'OCDE sur le financement de l'entrepreneuriat, le capital-risque correspond à la somme $\mathrm{du}$ capital-risque investi au stade de développement initial (qui comprend le préamorçage, l'amorçage, le démarrage et les autres stades de développement initial) et au stade de développement ultérieur. Comme les diverses associations d'investisseurs de capital-risque et autres fournisseurs de données n'utilisent pas de définition harmonisée des stades d'investissement du capital-risque, les données d'origine ont été agrégées à nouveau de manière à correspondre à la classification du capital-risque par stades de développement de l'OCDE. L'Afrique du Sud, la Corée, la Fédération de Russie et la Nouvelle-Zélande ne fournissent pas de ventilations du capital-risque par stade de développement qui permettraient de réaliser des comparaisons internationales valables.

L'annexe C présente la liste des sources de données par pays ; le tableau C.2 présente les correspondances entre les données d'origine et les données harmonisées par l'OCDE de la ventilation du capital-risque par stade de développement.

\section{Source}

Base de données de l'OCDE sur le financement de l'entrepreneuriat.

\section{Pour en savoir plus}

OCDE (2017), Financing SMEs and Entrepreneurs 2017: An OECD Scoreboard, Éditions OCDE, Paris, http://dx.doi.org/10.1787/ fin_sme_ent-2017-en.

OCDE (2015), New Approaches to SME and Entrepreneurship Financing: Broadening the Range of Instruments, Éditions OCDE, Paris, http://dx.doi.org/10.1787/9789264240957-en. 
Graphique 7.1. Investissements de capital-risque en pourcentage du PIB En pourcentage, 2016 ou dernière année disponible

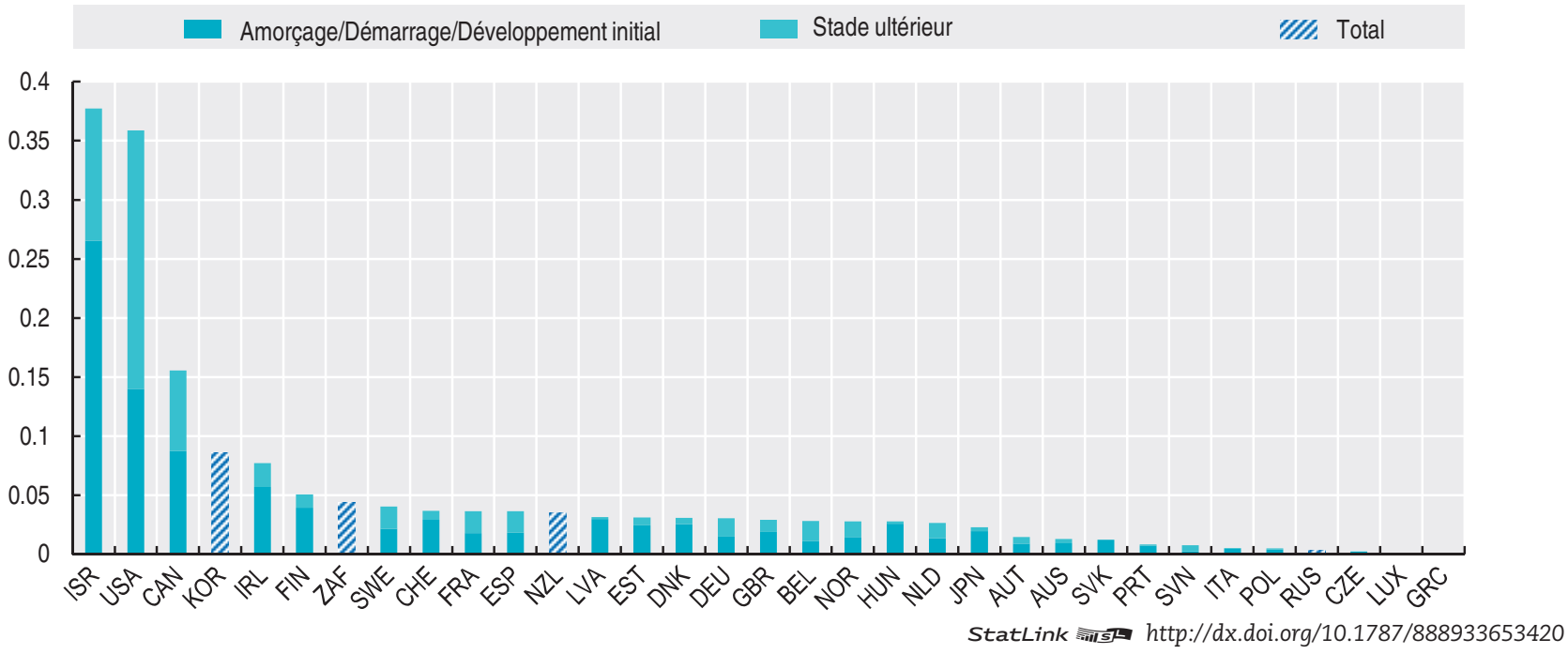

Graphique 7.2. Tendances en matière d'investissements de capital-risque Indice $2010=100$

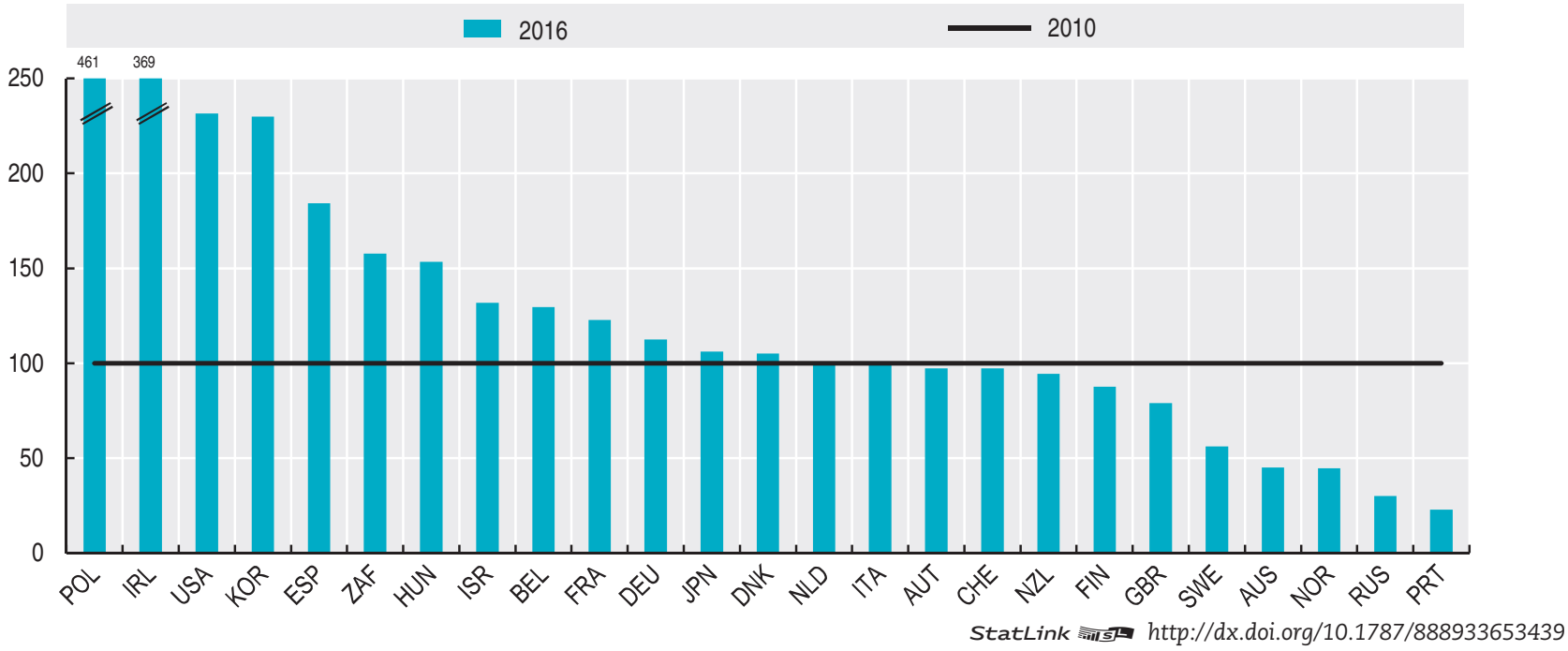

Tableau 7.1. Investissements de capital-risque

En millions USD, 2016 ou dernière année disponible

\begin{tabular}{|c|c|c|c|c|c|}
\hline Grèce & 0.00 & Nouvelle-Zélande & 64.25 & Espagne & 446.52 \\
\hline Luxembourg & 0.79 & Danemark & 93.87 & Royaume-Uni & 761.36 \\
\hline Slovénie & 3.28 & Italie & 96.84 & France & 894.22 \\
\hline République tchèque & 4.65 & Norvège & 103.00 & Allemagne & 1051.38 \\
\hline Estonie & 7.20 & Finlande & 119.65 & Israël (2014) & 1165.00 \\
\hline Lettonie & 8.73 & Afrique du Sud & 129.61 & Corée & 1212.22 \\
\hline République slovaque & 11.03 & Belgique & 130.73 & Japon & 1367.77 \\
\hline Portugal & 16.71 & Australie & 165.77 & Canada & 2377.40 \\
\hline Pologne & 23.71 & Pays-Bas & 204.10 & Total Europe & 4744.81 \\
\hline Hongrie & 34.57 & Suède & 206.61 & États-Unis & 66626.56 \\
\hline Fédération de Russie & 46.00 & Irlande & 226.94 & & \\
\hline Autriche & 55.87 & Suisse & 243.04 & & \\
\hline
\end{tabular}




\section{À savoir}

- Le nombre d'entreprises soutenues financièrement à l'aide de capital-risque est très faible, et représente généralement un pourcentage minime du total des naissances d'entreprises au cours d'une année donnée. Dans la plupart des pays de l'OCDE, les entreprises financées à l'aide de capital-risque ont représenté moins de $1 \%$ du total des naissances d'entreprises en 2016.

- En 2016, l'investissement moyen par entreprise a été supérieur à 5 millions USD au Canada, aux États-Unis, en Irlande et en Israël, alors que dans les quatre grandes économies de la zone euro l'Allemagne, l'Espagne, la France et l'Italie, il a été compris dans une fourchette de 1 à 2 millions USD.

- De 2007 à 2016, le pourcentage des investissements de capitalrisque dans des entreprises de moins de 20 salariés est passé de $30 \%$ à $40 \%$ en Europe.

\section{Pertinence}

Le capital-risque est une forme de capital-investissement particulièrement importante pour les jeunes entreprises présentant un potentiel d'innovation et de croissance, mais dont le modèle économique n'a pas encore fait ses preuves et qui n'ont pas d'historique d'activité ; il remplace ou complète les financements bancaires classiques. Le développement de cette forme de financement est considéré comme une condition-cadre importante pour stimuler l'entrepreneuriat innovant.

\section{Définitions}

Les entreprises financées par le capital-risque (sociétés de portefeuille ou sociétés émettrices) sont de nouvelles ou jeunes entreprises qui sont (en partie ou totalement) financées par du capital-risque.

Les entreprises financées par le capital-risque à différents stades de développement correspondent aux pourcentages d'entreprises financées par du capital-risque en fonction de leur stade de développement, tel qu'harmonisé par l'OCDE (préamorçage/ amorçage ; démarrage/autre stade de développement initial ; stade de développement ultérieur). Voir tableau C.2, annexe C).

La moyenne des investissements de capital-risque par entreprise est le ratio entre le total des investissements de capital-risque dans un pays et le nombre d'entreprises financées par du capital-risque dans ce pays.

La proportion d'entreprises financées par du capital-risque est obtenue en calculant le nombre d'entreprises qui ont reçu du capital-risque sur 1000 naissances d'entreprises employant des salarié.

La tendance-cycle rend compte sous une forme combinée des mouvements de long terme (tendance) et des mouvements de moyen à long terme (cycle) dans la série originale (voir http:// stats.oecd.org/glossary/detail.asp?ID=6693).

Informations sur les données concernant Israël : http://dx.doi. org/10.1787/888932315602.

\section{Comparabilité}

Il n'existe pas de définition internationale standard du capital-risque ou de la ventilation des investissements correspondants en fonction du stade de développement. De surcroît, la méthode de collecte des données diffère selon les pays.

Les données relatives au capital-risque proviennent principalement d'associations nationales ou régionales d'investisseurs de capital- risque qui les produisent elles-mêmes, dans certains cas avec le soutien de fournisseurs de données commerciales, à l'exception de l'Australie, où les statistiques sur le capital-risque sont recueillies et publiées par l'office statistique national australien.

Les statistiques présentées correspondent à l'agrégation de données sur les investissements en fonction de la localisation des sociétés de portefeuille, quelle que soit celle des sociétés de capitalinvestissement, sauf pour l'Australie, la Corée et le Japon, où les données se rapportent au lieu d'implantation des sociétés de capital-risque.

Pour Israël, les données concernent uniquement les entreprises financées par du capital-risque qui appartiennent au secteur de la haute technologie. Les données relatives aux États Unis englobent aussi les investissements de capital-risque réalisés par des entreprises qui ne sont pas des sociétés de capital-risque, à l'exclusion des investissements financés à $100 \%$ par des entreprises et/ou des business angels ; de plus, les données se réfèrent au nombre d'opérations plutôt qu'au nombre d'entreprises bénéficiaires. Celles relatives à l'Australie, au Japon et à la Nouvelle Zélande font référence à l'année budgétaire. Pour l'Europe, elles incluent seulement les investissements de capital-risque (amorçage, démarrage et phase ultérieure de développement) réalisés par des gestionnaires de fond conventionnels tels que les fonds de capital-investissement réalisant des investissements directs, les fonds mezzanine de capitalinvestissement, les fonds de co-investissement ou les fonds de sauvetage/de retournement ; les investissements réalisés par des business angels, des incubateurs, des fonds d'infrastructure, des fonds immobiliers, des fonds de créances sinistrées, des fonds de fonds primaires ou les fonds de fonds secondaires sont exclus de ces données ; le montant des investissements ne rend compte que du montant des capitaux propres investis par les gestionnaires de fond conventionnels et non de la valeur du cycle de financement dans son ensemble. Le capital-développement ou le capital destiné à financer le rachat d'entreprises par leurs cadres ou leurs salariés, actuellement ou auparavant financées à l'aide de capital-risque ne sont pas non plus inclus.

Dans la Base de données de l'OCDE sur le financement de l'entrepreneuriat, le capital-risque correspond à la somme du capital-risque investi au stade de développement initial (qui comprend le préamorçage, l'amorçage, le démarrage et les autres stades de développement initial) et au stade de développement ultérieur. Comme les diverses associations d'investisseurs de capital-risque et autres fournisseurs de données n'utilisent pas de définition harmonisée des stades d'investissement du capital-risque, les données d'origine ont été agrégées à nouveau de manière à correspondre à la classification du capital-risque par stades de développement de l'OCDE. L'Afrique du Sud, la Corée, la Fédération de Russie et la Nouvelle-Zélande ne fournissent pas de ventilations du capital-risque par stade de développement qui permettraient de réaliser des comparaisons internationales valables.

L'annexe C présente la liste des sources de données par pays ; le tableau C.2 présente les correspondances entre les données d'origine et les données harmonisées par l'OCDE de la ventilation du capital-risque par stade de développement.

\section{Source}

Base de données de l'OCDE sur le financement de l'entrepreneuriat.

\section{Pour en savoir plus}

OCDE (2017), Financing SMEs and Entrepreneurs 2017: An OECD Scoreboard, Éditions OCDE, Paris. http://dx.doi.org/10.1787/ fin_sme_ent-2017-en.

OCDE (2015), New Approaches to SME and Entrepreneurship Financing: Broadening the Range of Instruments, Éditions OCDE, Paris. http://dx.doi.org/10.1787/9789264240957-en. 
Graphique 7.3. Entreprises financées par le capital-risque à différents stades de développement En pourcentage, 2016 ou dernière année disponible

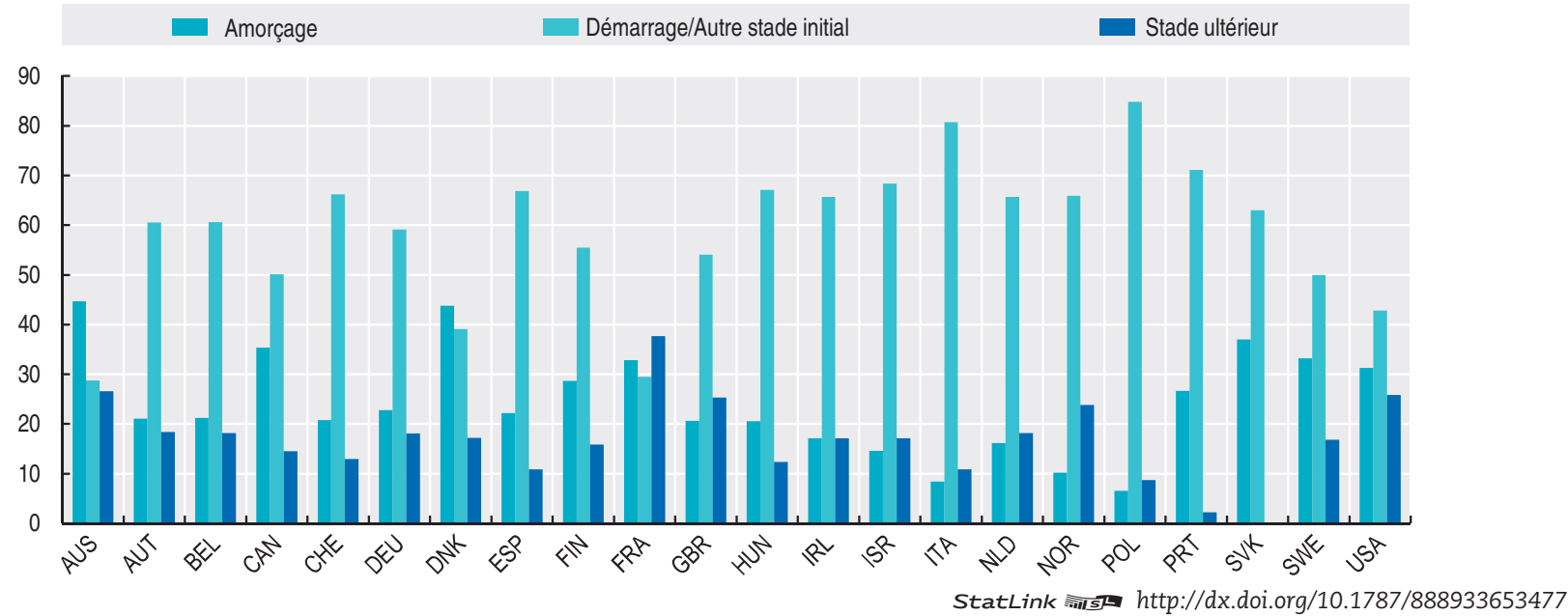

Graphique 7.4. Moyenne des investissements de capital-risque par entreprise En millions USD

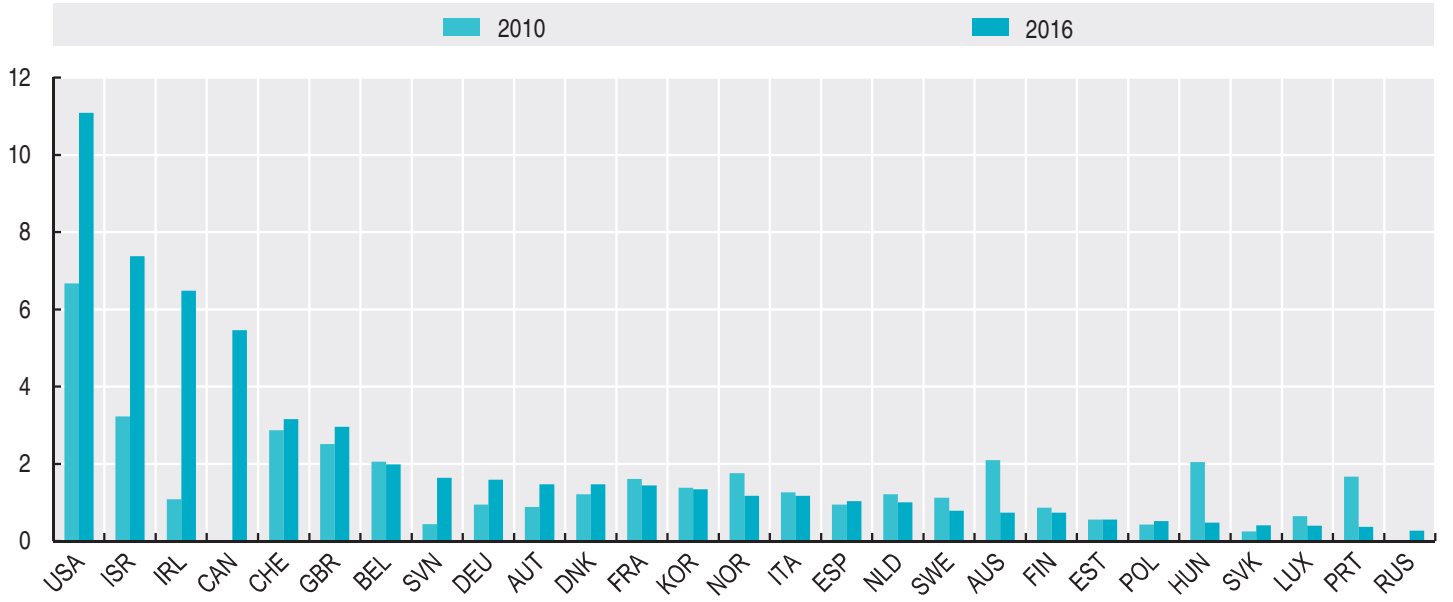

StatLink 解象 http://dx.doi.org/10.1787/888933653496

Graphique 7.5. Proportion d'entreprises financées par du capital-risque

Pour 1000 naissances d'entreprises employant des salariés

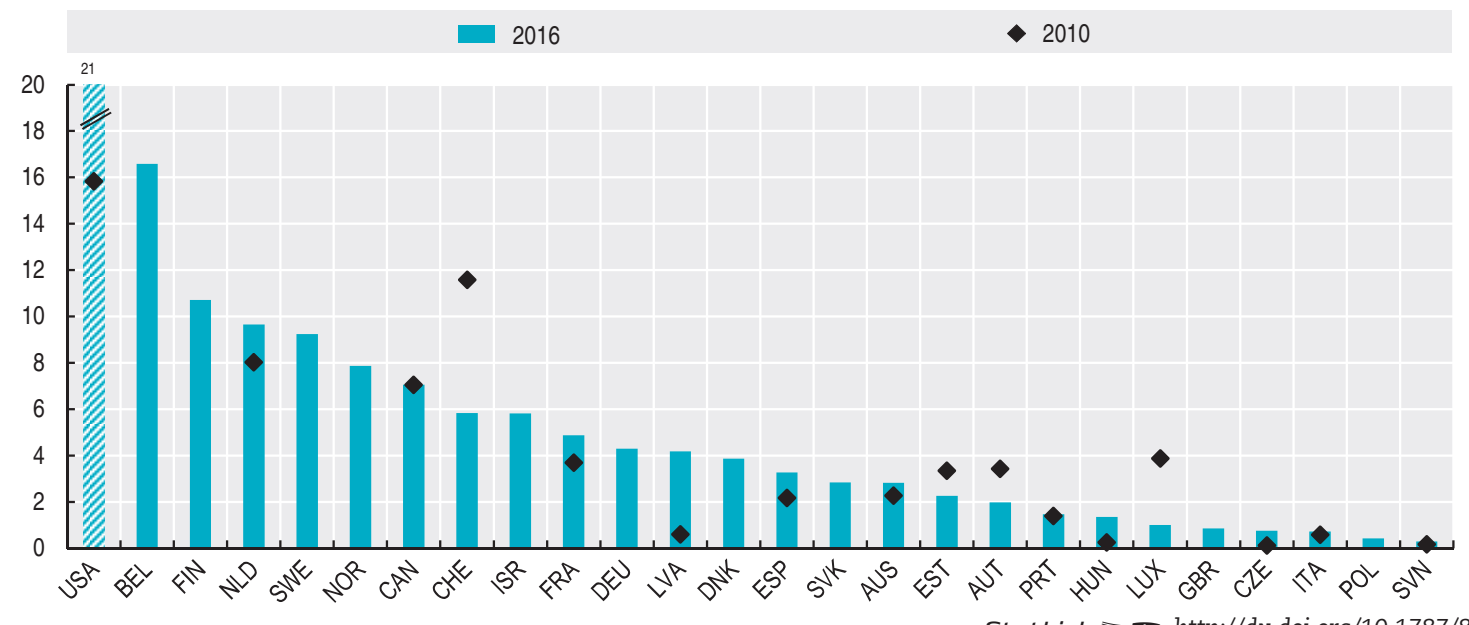

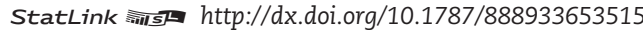




\section{CAPITAL-RISQUE}

Investissements de capital-risque par entreprise bénéficiaire

Tableau 7.2. Nombre d'entreprises financées par du capital-risque

\begin{tabular}{|c|c|c|c|c|c|}
\hline Pays & 2010 & 2016 & Pays & 2010 & 2016 \\
\hline Grèce & 2 & 0 & Italie & 77 & 83 \\
\hline Luxembourg & 7 & 2 & Norvège & 131 & 88 \\
\hline Slovénie & 1 & 2 & Israël (2014) & 274 & 158 \\
\hline République tchèque & 3 & 9 & Finlande & 159 & 164 \\
\hline Estonie & 15 & 13 & Pays-Bas & 169 & 204 \\
\hline Lettonie & 4 & 23 & Australie & 176 & 226 \\
\hline République slovaque & 11 & 27 & Royaume-Uni & 383 & 257 \\
\hline Irlande & 57 & 35 & Suède & 329 & 262 \\
\hline Autriche & 65 & 38 & Espagne & 258 & 432 \\
\hline Portugal & 44 & 45 & Canada & 344 & 435 \\
\hline Pologne & 12 & 46 & France & 452 & 621 \\
\hline Danemark & 74 & 64 & Allemagne & 991 & 663 \\
\hline Belgique & 49 & 66 & Corée & 382 & 902 \\
\hline Hongrie & 11 & 73 & États-Unis & 4311 & 6009 \\
\hline Suisse & 87 & 77 & & & \\
\hline
\end{tabular}

Graphique 7.6. Évolution des investissements de capital-risque par taille d'entreprise financée par du capital-risque, Europe

En pourcentage du total des investissements de capital-risque dans les PME

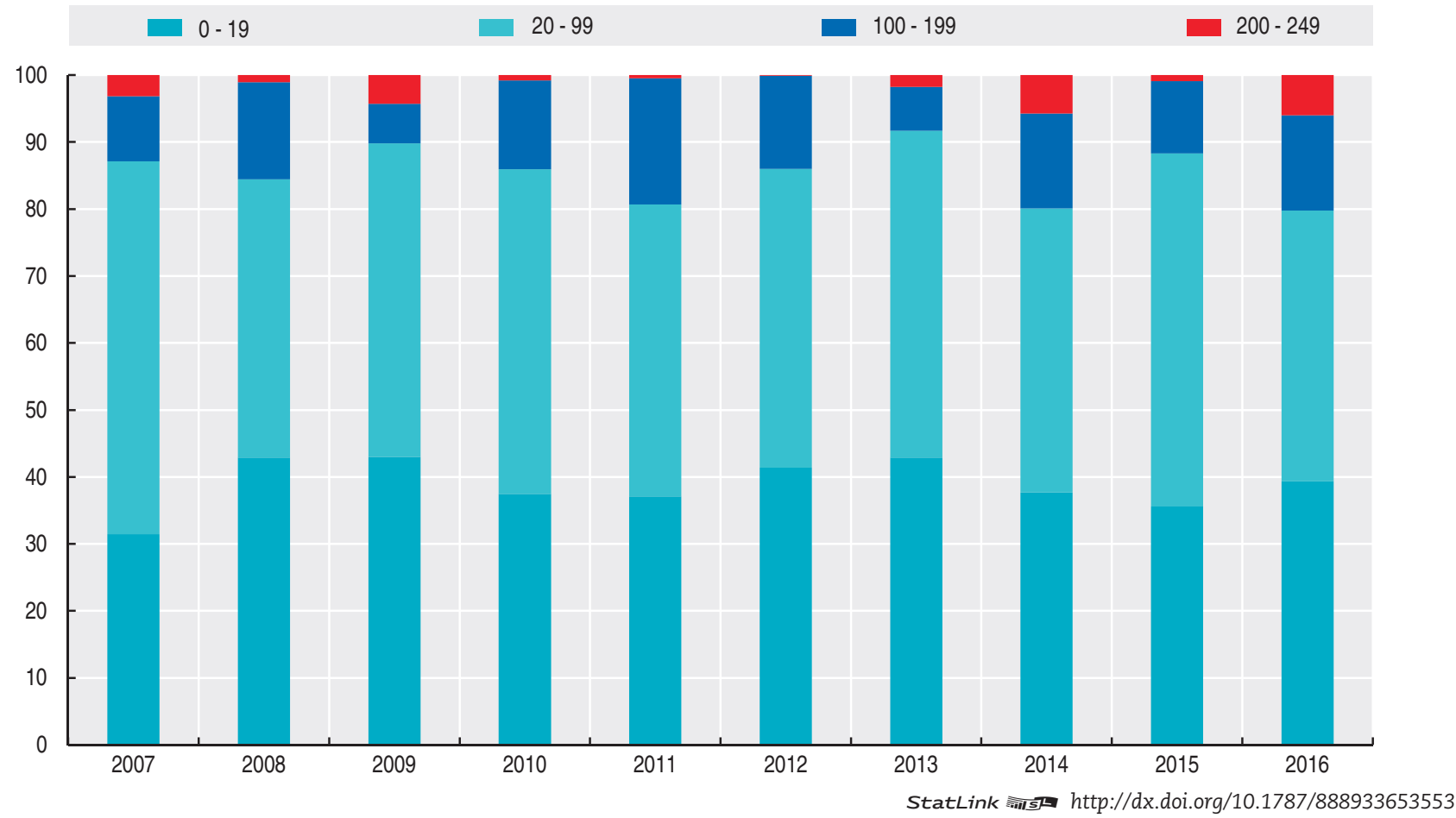


Graphique 7.7. Investissements de capital-risque, Europe

Tendance-cycle, $2010=100$

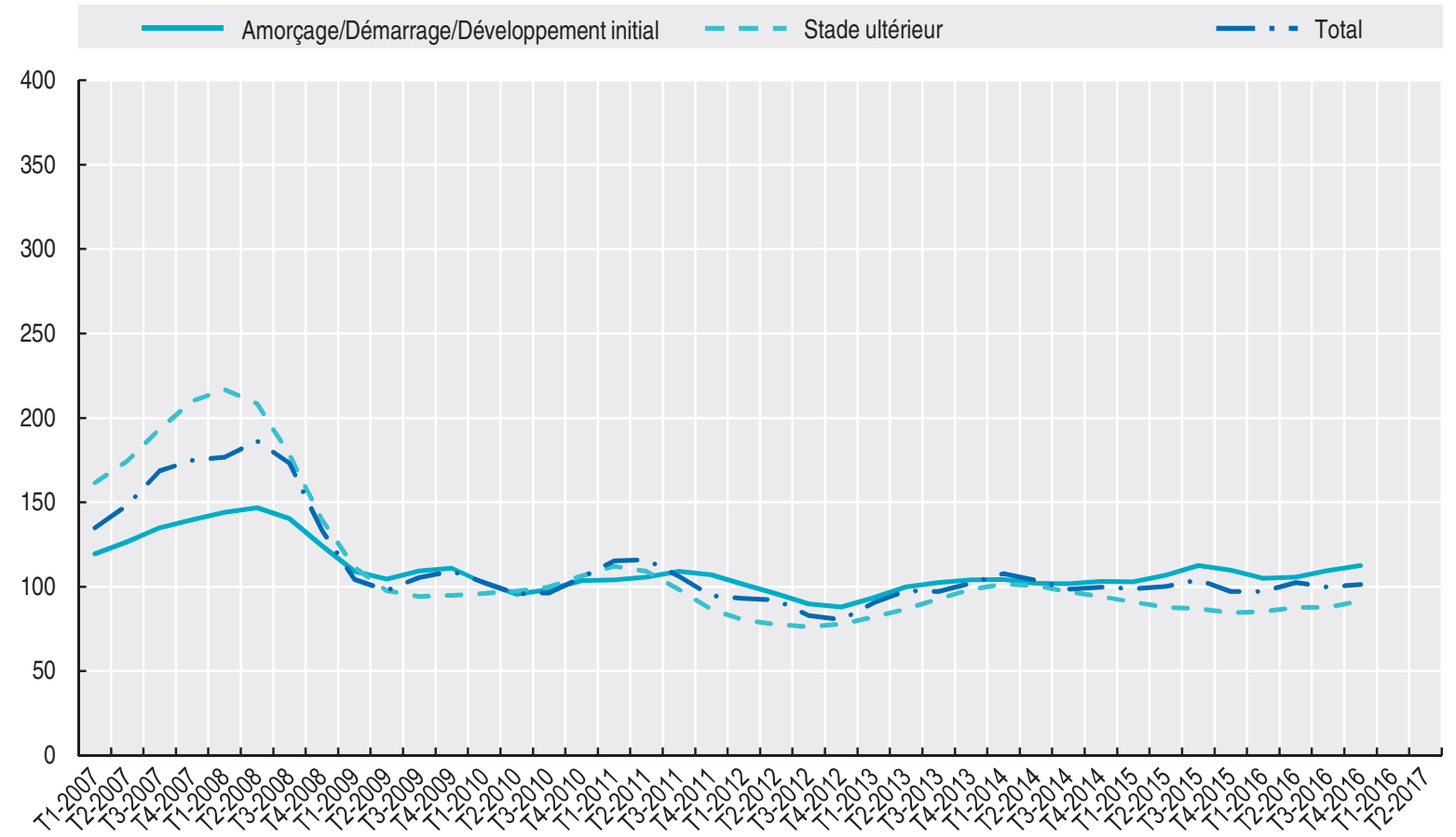

StatLink ailst http://dx.doi.org/10.1787/888933653572

Graphique 7.8. Investissements de capital-risque, États-Unis

Tendance-cycle, $2010=100$

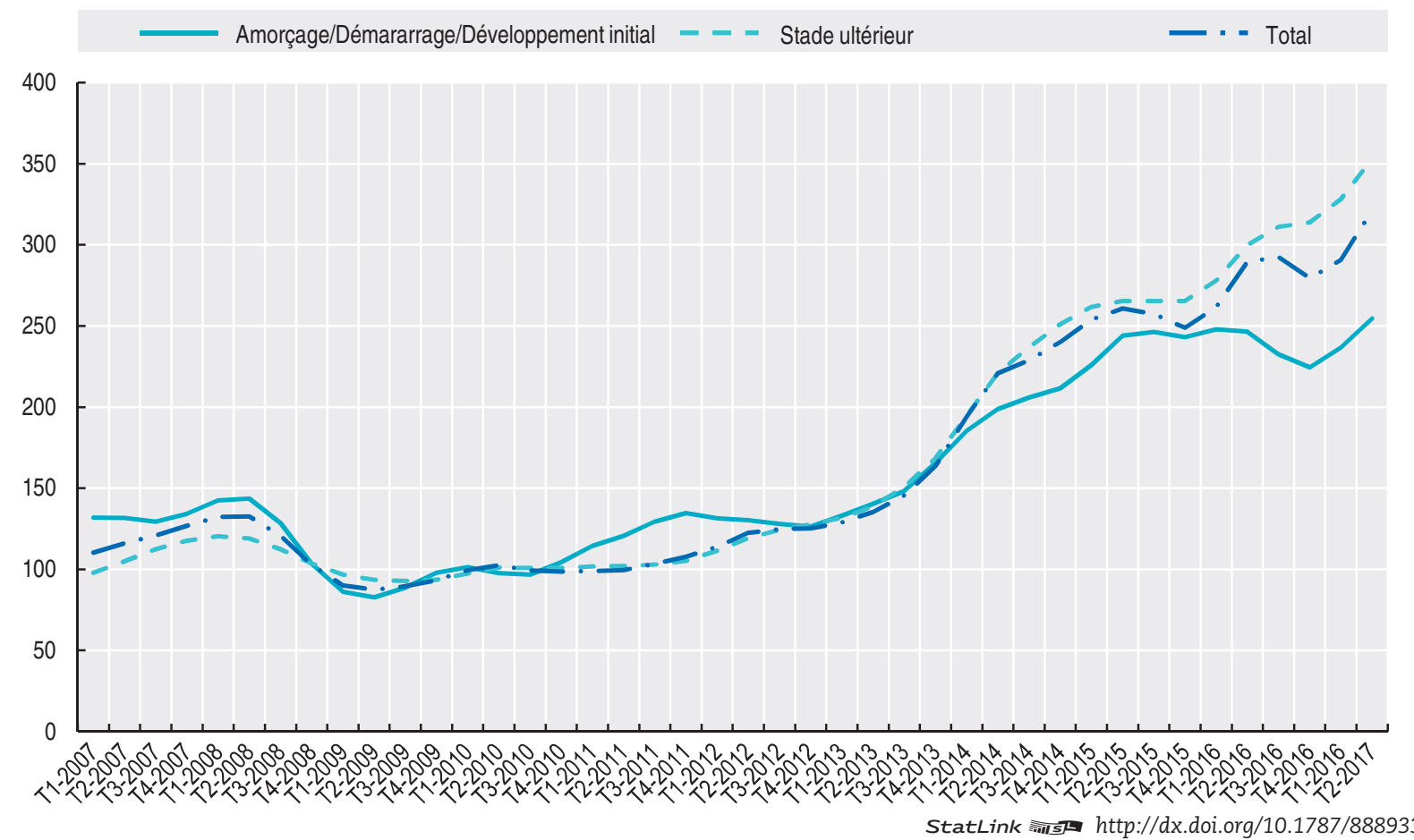

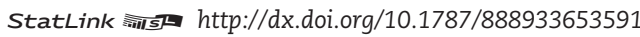




\section{À savoir}

- En 2016, aux États-Unis, le secteur des technologies de l'information et des communications a concentré plus de la moitié du total des investissements de capital-risque (53.6\%), suivi par les sciences de la vie (20.7\%). En Europe, le secteur qui a attiré le plus d'investissements de capital-risque a été celui des technologies de l'information et des communications (44\% du total), suivi par les sciences de la vie (27\%).

- De 2007 à 2016, l'écart qui existait entre les États-Unis et l'Europe en ce qui concerne les investissements de capitalrisque s'est accentué dans tous les secteurs.

\section{Pertinence}

Le capital-risque est une forme de capital-investissement particulièrement importante pour les jeunes entreprises présentant un potentiel d'innovation et de croissance, mais dont le modèle économique n'a pas encore fait ses preuves et qui n'ont pas d'historique d'activité ; il remplace ou complète les financements bancaires classiques. L'objectif du capital-risque est d'obtenir des rendements élevés à partir d'investissements initiaux peu importants, principalement dans des secteurs ayant de faibles besoins de fonds propres, comme par exemple les TIC ou les sciences de la vie. À l'opposé, les secteurs ayant des exigences de fonds propres plus importantes - comme l'immobilier et l'exploitation minière - recueillent comparativement des investissements de capital-risque plus réduits.

\section{Définitions}

Le capital-risque est un sous-ensemble du capitalinvestissement (c'est-à-dire des fonds propres fournis à des entreprises non cotées en bourse) et désigne les investissements en fonds propres réalisés pour financer les phases de prédémarrage, de démarrage et de développement initial d'une entreprise (Source : Invest Europe).

\section{Comparabilité}

Il n'existe pas de définition internationale standard du capitalrisque ou de la ventilation des investissements correspondants en fonction du stade de développement. De surcroît, la méthode de collecte des données diffère selon les pays.

Les données relatives au capital-risque proviennent principalement d'associations nationales ou régionales d'investisseurs de capital-risque qui les produisent ellesmêmes, dans certains cas avec le soutien de fournisseurs de données commerciales, à l'exception de l'Australie, où les statistiques sur le capital-risque sont recueillies et publiées par l'office statistique national australien.

Les données relatives aux États Unis englobent aussi les investissements de capital-risque réalisés par des entreprises qui ne sont pas des sociétés de capital-risque, à l'exclusion des investissements financés à $100 \%$ par des entreprises et/ou des business angels. Pour l'Europe, elles incluent seulement les investissements de capital-risque (amorçage, démarrage et phase ultérieure de développement) réalisés par des gestionnaires de fond conventionnels tels que les fonds de capital-investissement réalisant des investissements directs, les fonds mezzanine de capitalinvestissement, les fonds de co-investissement ou les fonds de sauvetage/de retournement; les investissements réalisés par des business angels, des incubateurs, des fonds d'infrastructure, des fonds immobiliers, des fonds de créances sinistrées, des fonds de fonds primaires ou les fonds de fonds secondaires sont exclus de ces données ; le montant des investissements ne rend compte que du montant des capitaux propres investis par les gestionnaires de fond conventionnels et non de la valeur $\mathrm{du}$ cycle de financement dans son ensemble. Le capitaldéveloppement ou le capital destiné à financer le rachat d'entreprises par leurs cadres ou leurs salariés, actuellement ou auparavant financées à l'aide de capital-risque, ne sont pas non plus inclus.

Dans la Base de données de l'OCDE sur le financement de l'entrepreneuriat, le capital-risque correspond à la somme du capital-risque investi au stade de développement initial (qui comprend le préamorçage, l'amorçage, le démarrage et les autres stades de développement initial) et au stade de développement ultérieur. Comme les diverses associations d'investisseurs de capital-risque et autres fournisseurs de données n'utilisent pas de définition harmonisée des stades d'investissement du capital-risque, les données d'origine ont été agrégées à nouveau de manière à correspondre à la classification du capital-risque par stades de développement de l'OCDE. L'Afrique du Sud, la Corée, la Fédération de Russie et la Nouvelle-Zélande ne fournissent pas de ventilations du capital-risque par stade de développement qui permettraient de réaliser des comparaisons internationales valables.

L'annexe C.3, annexe C, présente les correspondances entre les données d'origine et les données harmonisées par l'OCDE de la ventilation du capital-risque par secteur.

\section{Sources}

Base de données de l'OCDE sur le financement de l'entrepreneuriat, le financement de l'entrepreneuriat, constituée à partir de :

Invest Europe, Invest Europe Yearbook - 2016 European Private Equity Activity, http://www.investeurope.eu/knowledgecenter/ statisticsdetail.aspx?id=6392.

NVCA (National Venture Capital Association, United States)/ PitchBook data, http://www.nuca.org/.

\section{Pour en savoir plus}

OCDE (2017), Financing SMEs and Entrepreneurs 2017: An OECD Scoreboard, Éditions OCDE, Paris. http://dx.doi.org/10.1787/ fin_sme_ent-2017-en.

OCDE (2015), Perspectives de l'économie numérique de l'OCDE 2015, Éditions OCDE, Paris. http://dx.doi.org/10.1787/ 9789264232440-en. 
Graphique 7.9. Investissements de capital-risque aux États-Unis, par secteur

En pourcentage, 2016

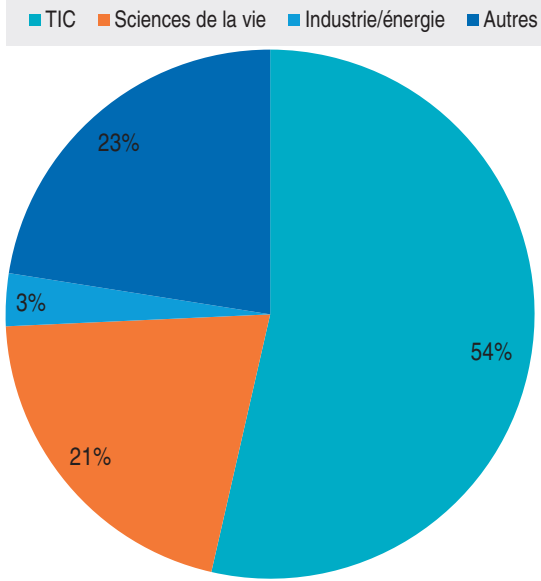

StatLink Aillsta $h t t p: / / d x . d o i . o r g / 10.1787 / 888933653610$
Graphique 7.10. Investissements de capital-risque en Europe, par secteur

En pourcentage, 2016

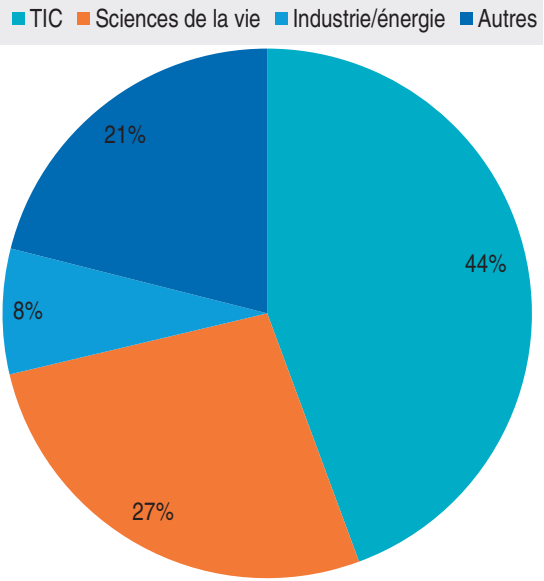

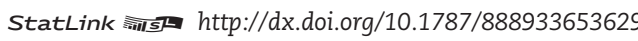

Graphique 7.11. Investissements de capital-risque par secteur, échantillon de pays européens En pourcentage, 2016

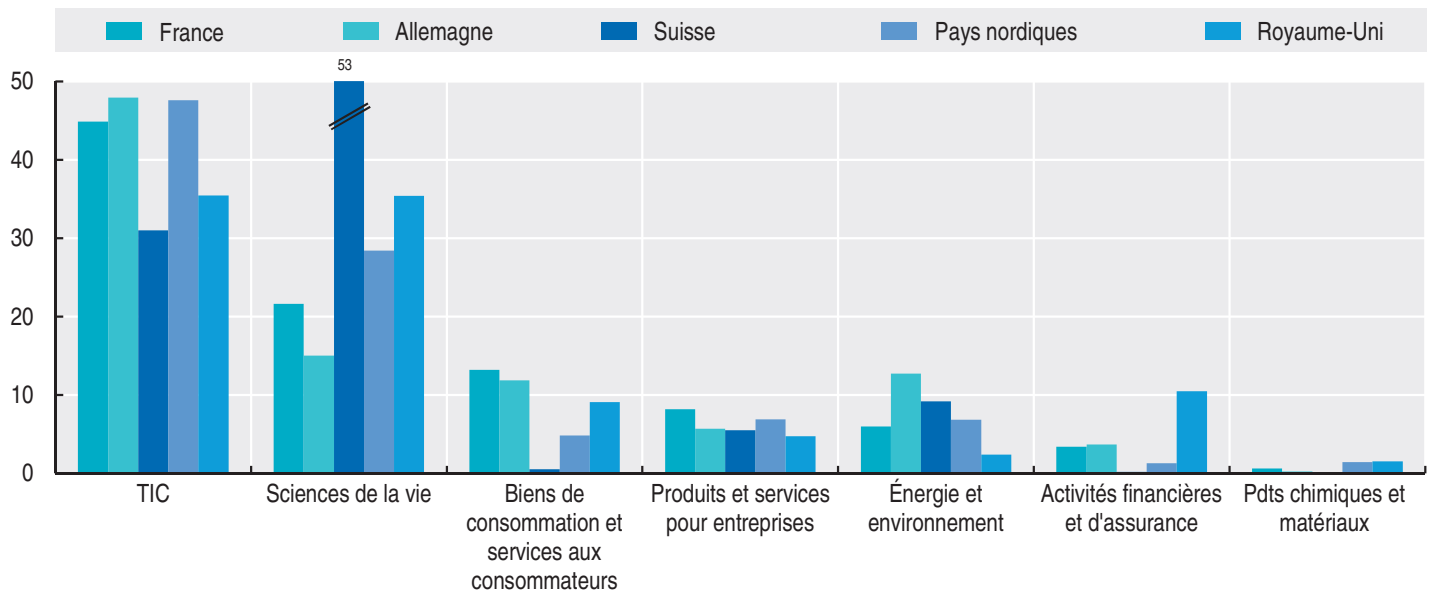

StatLink *iाls http://dx.doi.org/10.1787/888933653648

Graphique 7.12. Investissements de capital-risque par secteur En million US dollars

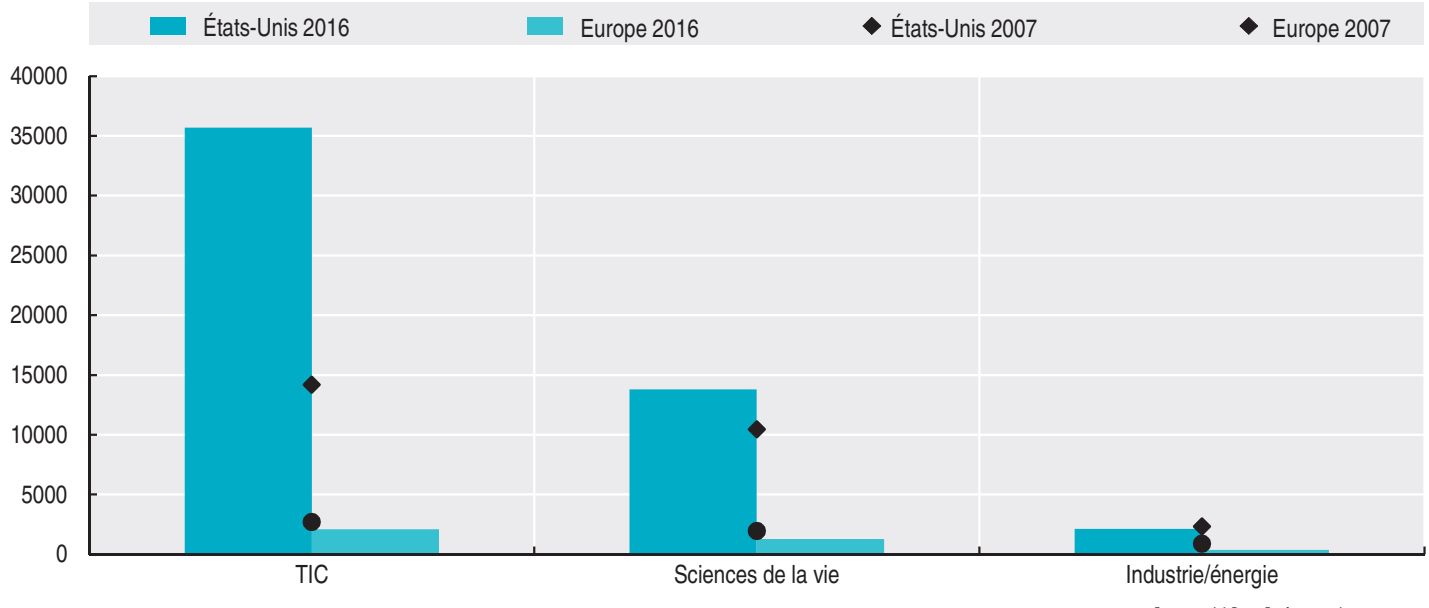





\section{Sources des données sur les indicateurs actualisés de l'entrepreneuriat}

La présente annexe répertorie les sources et les définitions utilisées pour élaborer la base de données de l'OCDE concernant les indicateurs actualisés de l'entrepreneuriat. Cette base contient des donnés infra-annuelles sur les créations et les faillites d'entreprises ; et elle est accessible à l'adresse suivante : http://stats.oecd.org//Index.aspx?QueryId=72208.

Les données sur les créations d'entreprises proviennent de diverses sources administratives et statistiques (tableau A.1), dont les définitions et la couverture varient considérablement d'un pays à l'autre et peuvent différer des concepts et du champ des définitions de référence des naissances fournis par le Manuel Eurostat-OCDE sur les statistiques démographiques des entreprises. Par exemple, les créations d'entreprises peuvent inclure de nouvelles entreprises créées à la suite de fusions, dissolutions, scissions et réactivations d'entreprises dormantes, en plus des naissances pures.

Les données administratives et statistiques sous-jacentes peuvent différer significativement selon les pays, notamment au niveau de la population des entreprises couvertes : type de forme juridique considéré (par exemple, entreprises constituées en sociétés uniquement), secteurs d'activité (couverture des activités agricoles ou éducatives) ou entreprises en-deçà d'un certain seuil de chiffre d'affaires ou d'emploi. Par exemple, les données administratives sous-jacentes pour l'Espagne excluent les personnes physiques et les entreprises individuelles et les données pour le Royaume-Uni excluent les sociétés non constituées en sociétés.

Les données sous-jacentes peuvent également être volatiles, car le périmètre des entreprises peut changer au cours du temps. Par exemple, pour le Royaume-Uni, les données de 2009 incluent également l'Irlande du Nord; et pour la Suède, des changements méthodologiques ont été introduits en 2010. Les changements de politique envers des formes particulières d'entreprises (notamment le statut juridique) peuvent également avoir un impact considérable sur les données brutes, en particulier si la politique favorise un changement de forme juridique envers les entreprises couvertes dans les données administratives brutes, au détriment des formes juridiques non couvertes (ou vice versa). Par exemple en France, un nouveau statut d'entreprise individuelle (régime de l'autoentrepreneur) a été mis en place en janvier 2009.

Les données sur les faillites (tableau A.2) proviennent de sources administratives brutes. Lorsque cela est possible, les données brutes sont adaptées pour garantir que la couverture sectorielle reflète la norme utilisée dans cette publication, c'est-à-dire que seul le secteur marchand est pris en compte. Les faillites sont basées sur les cadres juridiques et 
institutionnels en place. Une différence clé avec la mesure du décès de l'entreprise examinée dans cette publication est qu'une entreprise « en faillite " peut continuer à fonctionner.

Les données sur les faillites sont affectées par des différences entre les législations nationales. Dans certains pays, une déclaration de faillite signifie que l'entreprise doit cesser immédiatement ses activités et qu'elle est donc plus proche du concept du décès de l'entreprise utilisé dans cette publication. Dans d'autres pays, cependant, les entreprises sont en mesure de continuer à négocier avec des mandataires liquidateurs prenant leur contrôle opérationnel, même après une déclaration officielle de faillite. En effet, certaines des entreprises se déclarant en faillite pourraient éventuellement se rétablir. La proportion des procédures de faillite qui aboutissent à des liquidations réelles (décès) des entreprises, et non à des réorganisations, varie selon les pays en fonction du code de la faillite. Au sujet des comparaisons avec les décès d'entreprises, il convient de préciser également que toutes les entreprises ne se déclarent pas en faillite avant leur fermeture (décès).

En raison de ces problèmes de comparabilité, les comparaisons internationales des données sur les créations et les faillites portent sur les changements de niveaux plutôt que sur les niveaux eux-mêmes. Les tendances sont calculées pour les créations et les faillites ; plus précisément, la tendance-cycle reflète les mouvements à la fois de long terme (tendance) et de moyen-long terme (cycle) de la série originale (voir : http://stats.oecd.org/glossary/detail. asp?ID=6693).

\section{Tableau A.1. Sources et définitions nationales des créations d'entreprises}

\begin{tabular}{|c|c|}
\hline & ources et définitions des créations d'entreprises \\
\hline Allemagne & $\begin{array}{l}\text { Source : Statistiches Bundesamt - Destatis. } \\
\text { Données mensuelles. } \\
\text { Nombre de nouvelles créations (sièges sociaux et établissements secondaires). Les petites unités et les activités auxiliaires ne sont pas incluses. La } \\
\text { transformation, le rachat et le changement de propriétaire sont exclus. Les nouvelles entreprises en provenance de l'étranger sont également retirées } \\
\text { des données concernant les naissances. } \\
\text { Toutes les activités sont prises en compte. } \\
\text { www.destatis.de }\end{array}$ \\
\hline Australie & $\begin{array}{l}\text { Source : Australian Business Register. } \\
\text { Données trimestrielles. } \\
\text { Les données couvrent toutes les entreprises en fonction de la date d'enregistrement initiale. Les données comprennent également les particuliers / } \\
\text { commerçants indépendants qui sont principalement des commerçants et des professionnels qui exploitent leur propre entreprise (non constituée en } \\
\text { société), les sociétés de personnes et les fiducies qui sont des structures d'entreprise non constituées en société (non enregistrées auprès de l'ASIC), } \\
\text { les sociétés constituées en société (enregistrées auprès de l'ASIC). Les entités gouvernementales (fédérales, étatiques et locales) et les caisses de } \\
\text { retraite sont également incluses car elles doivent avoir un identifiant ABN. }\end{array}$ \\
\hline Belgique & $\begin{array}{l}\text { Source : Statistics Belgium. } \\
\text { Données mensuelles. } \\
\text { Ces statistiques sont élaborées par Statistics Belgium à partir de la Banque-Carrefour des Entreprises. Les données concernent les personnes } \\
\text { (physiques et morales) assujetties à la TVA. } \\
\text { http://statbel.fgov.be/fr }\end{array}$ \\
\hline Canada & $\begin{array}{l}\text { Source : Statistique Canada. } \\
\text { Données trimestrielles. } \\
\text { Les données proviennent des estimations trimestrielles expérimentales de la dynamique des entreprises par secteur établies à partir des données des } \\
\text { retenues sur la paye (PD7). Les statistiques annuelles sur l'entrée et la sortie d'entreprises sont élaborées à partir des états de la rémunération payée } \\
\text { (feuillets T4). Ces feuillets contiennent des informations à la fois sur les employeurs et les salariés, ce qui permet de suivre les individus lorsqu'ils } \\
\text { changent d'entreprise, et de limiter les fausses naissances d'entreprises. } \\
\text { http://www.statcan.gc.ca/fra/debut }\end{array}$ \\
\hline Danemark & $\begin{array}{l}\text { Source : Danish Business Authority. } \\
\text { Données mensuelles. } \\
\text { Les données recouvrent toutes les formes juridiques d'entreprises (y compris les entrepreneurs individuels) et l'ensemble de l'économie (y compris } \\
\text { l'agriculture). Le décompte des nouvelles entreprises inclut les changements de secteur d'activité ou d'adresse, mais pas les fusions ni les entreprises } \\
\text { créées par essaimage, sauf si elles s'accompagnent d'un changement de secteur ou d'adresse. } \\
\text { www.cvr.dk }\end{array}$ \\
\hline
\end{tabular}




\section{Tableau A.1. Sources et définitions nationales des créations d'entreprises (suite)}

\begin{tabular}{ll}
\hline Sources and definitions of enterprise creation \\
\hline Espagne & Source : Instituto Nacional de Estadistica de Espana (INE) et Central Business Register (CBR). \\
& Données mensuelles. \\
& Nombre d'entrées. \\
& Le registre « Mercantile Companies » contient des informations sur les entreprises marchandes et constituées en sociétés (mais pas sur les personnes \\
& physiques ou les entrepreneurs individuels). Les « sociétés commerciales créées » peuvent ne pas être actives et les « sociétés commerciales \\
& dissoutes » peuvent être retirées du registre sans jamais avoir été actives. \\
& www.ine.es/en/
\end{tabular}

États-Unis Source : Bureau of Labor Statistics (BLS) - Business Employment Dynamics (BED).

Données trimestrielles.

Les données concernent les naissances d'établissements de toutes tailles dans les secteurs de production de biens et de prestation de services. Cela inclut les unités qui ont enregistré pour la première fois des créations d'emplois au troisième mois du trimestre en cours, sans lien avec le trimestre précédent ; ou les unités qui ont enregistré des créations d'emplois au troisième mois du trimestre en cours et aucune création au troisième mois des quatre précédents trimestres. Les naissances sont un sous-groupe des créations d'entreprises, qui n'inclut pas les réouvertures des entreprises exerçant une activité saisonnière.

www.bls.gov/datal

Fédération Source : Federal State Statistics Service.

de Russie Données mensuelles. Nouvelles inscriptions. http://www.gks.ru/bgd/regl/b17_01/Main.htm

Finlande Source : Statistics Finland.

Données trimestrielles.

Les statistiques sont établies à partir du registre des entreprises de Statistics Finland. Elles portent sur les entreprises exerçant des activités commerciales qui sont assujetties à la TVA ou qui emploient des salariés. Sont exclues les fondations, les sociétés immobilières, les associations de bienfaisance, les organismes publics et les communautés religieuses. Les statistiques prennent en compte les entreprises sous contrôle de l'État mais pas celles sous le contrôle des municipalités. Des données sont fournies concernant le nombre de créations d'entreprises.

http://www.stat.fi/til/aly/2014/aly_2014_2015-10-29_tie_001_en.htmI

France Source : INSEE, SIRENE.

Données mensuelles.

Nombre de naissances. On parle de naissance lorsqu'une combinaison de moyens de production est créée sans qu'une autre entreprise ne soit impliquée. Une rupture de série est à noter en 2009, du fait de la mise en œuvre d'un nouveau statut juridique d'entreprise individuelle (le régime de l'auto-entrepreneur). Depuis décembre 2014, les auto-entrepreneurs sont désignés sous une nouvelle dénomination, celle de " micro-entrepreneurs ». Hors agriculture.

http://www.insee.fr

Hongrie Source : Hungarian Central Statistical Office

Données mensuelles par forme juridique. Les entreprises enregistrées désignent les unités qui, d’après les enregistrements administratifs, existent légalement à la date de l'enquête et ont un numéro d'identification fiscale, y compris celles qui font l'objet d'une procédure de faillite, de liquidation et de dissolution à la date de référence.

https://www.ksh.hu/stadat_infra_3_2.

Islande Source: Statistics Iceland.

Données mensuelles.

Les données se réfèrent aux entreprises nouvellement enregistrées, dont le nombre est communiqué par l'Internal Revenue Directorate. www.statice.is

Italie $\quad$ Source : InfoCamere, Movimprese - Registre d'entreprises des chambres de commerce italiennes.

Données trimestrielles.

Nombre d'entrées (iscritte).

Toutes les formes juridiques et toutes les activités sont prises en compte.

www.infocamere.it

Norvège Source : Statistics Norway.

Données mensuelles.

Les données recouvrent l'ensemble de l'économie, sauf l'agriculture. Le décompte inclut également les entrepreneurs individuels. https://www.ssb.no

Nouvelle- $\quad$ Source : New Zealand Companies Office.

Zélande Données trimestrielles.

Les données recouvrent uniquement les entreprises constituées en sociétés.

Pays-Bas Source : Statistics Netherlands.

Données mensuelles.

Les données recouvrent l'ensemble de l'économie (hormis l'agriculture) et toutes les formes juridiques d'entreprises. Une création est définie comme l'émergence d'une nouvelle entreprise.

http://www.cbs.nl/

Portugal Source : Statistics Portugal.

Données mensuelles.

Les nouveaux enregistrements de personnes morales et d'entités équivalentes sont effectués par le ministère de la Justice (Direction générale de la politique en matière de justice).

www.ine.pt 
Tableau A.1. Sources et définitions nationales des créations d'entreprises (suite)

\begin{tabular}{ll}
\hline & Sources and definitions of enterprise creation \\
\hline Royaume-Uni & Source : Companies House. \\
& Données mensuelles. \\
& Nouvelles immatriculations (nombre d'entrées). \\
& Toutes les sociétés à responsabilité limitée d'Angleterre, du pays de Galles, d'Irlande du Nord et d'Écosse sont immatriculées auprès de la Companies \\
& House. \\
& Une entrée correspond à l'émergence d'une nouvelle entreprise dans l'économie, quel que soit l'évènement démographique qui en est à l'origine : \\
& fusion, changement de nom, scission ou naissance. www.gov.uk/government/statistics \\
& Source : Swedish Agency for Growth Policy Analysis. \\
& Données trimestrielles. \\
& Nombre d'entreprises nouvellement établies. Les données recouvrent l'ensemble de l'économie, y compris l'agriculture. \\
& www.tillvaxtanalys.se/ \\
& Source: The Union of Chambers and Commodity Exchanges of Turkey \\
& Données mensuelles de créations selon trois principales formes juridiques. \\
& http://tobb.org.tr/BilgiErisimMudurlugu/Sayfalar/Eng/KurulanKapananSirketistatistikleri.php. \\
Turquie &
\end{tabular}

\section{Tableau A.2. Sources et définitions nationales des faillites}

\begin{tabular}{|c|c|}
\hline Pays & Sources et définitions des faillites \\
\hline Afrique du Sud & $\begin{array}{l}\text { Source : Statistics South Africa. } \\
\text { Données mensuelles. } \\
\text { Statistiques sur les liquidations. } \\
\text { www.statssa.gov.za/ }\end{array}$ \\
\hline Allemagne & $\begin{array}{l}\text { Source: Statistiches Bundesamt - Destatis. } \\
\text { Données mensuelles. } \\
\text { Insolvabilités. } \\
\text { Les données concernent les entreprises et les travailleurs indépendants. } \\
\text { Toutes les activités sont prises en compte. } \\
\text { www.destatis.de/EN/Homepage.htmI }\end{array}$ \\
\hline Australie & $\begin{array}{l}\text { Source: Australian Securities and Investments Commission (ASIC). } \\
\text { Données mensuelles. } \\
\text { Statistiques d'insolvabilités - Entreprises placées sous administration externe. } \\
\text { Les statistiques sur les entreprises placées sous administration externe comptabilisent le nombre d'entreprises soumises pour la première fois à } \\
\text { une forme d'administration externe. L'ASIC précise qu'une entreprise ne sera incluse qu'une seule fois dans ces statistiques, qu'elle fasse ou non } \\
\text { l'objet d'une autre forme d'administration externe par la suite. La seule exception concerne les entreprises qui quittent le régime de l'administration } \\
\text { externe, par exemple du fait d'une décision de justice, et qui le réintègrent ultérieurement. Ces statistiques ne tiennent pas compte des liquidations } \\
\text { volontaires à l'initiative des associés eux-mêmes. } \\
\text { www.asic.gov.au }\end{array}$ \\
\hline Belgique & $\begin{array}{l}\text { Source: Statistics Belgium. } \\
\text { Données mensuelles. } \\
\text { Statistiques sur les faillites. } \\
\text { Statistics Belgium élabore ses statistiques à partir des déclarations des tribunaux de commerce et les complète, si nécessaire, par des informations } \\
\text { provenant de son registre des entreprises. Les données se réfèrent aux faillites de sociétés. } \\
\text { Toutes les activités économiques sont prises en compte. } \\
\text { http://statbel.fgov.be/fr/ }\end{array}$ \\
\hline Brésil & $\begin{array}{l}\text { Source: Serasa Experian. } \\
\text { Données mensuelles. } \\
\text { Les données incluent le nombre total de faillites prononcées et effectives, ainsi que le total des redressements judiciaires prononcés, différés et } \\
\text { effectifs. } \\
\text { http://www.serasaexperian.com.br/release/indicadores/falencias_concordatas.htm }\end{array}$ \\
\hline Canada & $\begin{array}{l}\text { Source: Bureau du surintendant des faillites du Canada. } \\
\text { Données mensuelles. } \\
\text { Une faillite d'entreprise est définie comme l'état d'une entreprise qui a demandé une procédure de faillite ou contre laquelle une demande de } \\
\text { mise en faillite a été prononcée. Une entreprise est définie comme toute entité ou organisation de nature commerciale autre qu'un individu, ou un } \\
\text { individu dont } 50 \% \text { ou plus de la dette totale est liée à l'exploitation d'une entreprise commerciale. } \\
\text { http://wWw.ic.gc.ca/eic/site/icgc.nsf/fra/accueil }\end{array}$ \\
\hline Espagne & $\begin{array}{l}\text { Source: Instituto Nacional de Estadística de España (INE). } \\
\text { The Mercantile Companies (MC) pour les données mensuelles. } \\
\text { Companies Central Directory (CCD) pour les données annuelles. } \\
\text { Nombre de sorties. } \\
\text { Le registre des sociétés commerciales comporte des informations sur les entreprises constituées en société (hors personnes physiques ou } \\
\text { entreprises individuelles). Les sociétés commerciales créées peuvent ne pas être actives et les sociétés marchandes dissoutes peuvent être retirées } \\
\text { du registre sans jamais avoir été actives. } \\
\text { www.ine.es }\end{array}$ \\
\hline
\end{tabular}


Tableau A.2. Sources et définitions nationales des faillites (suite)

\begin{tabular}{|c|c|}
\hline Pays & Sources et définitions des faillites \\
\hline États-Unis & $\begin{array}{l}\text { Source: tribunaux des États-Unis. } \\
\text { Données trimestrielles. } \\
\text { Statistiques des requêtes de mise en faillite - ensemble des déclarations d'entreprises (chapitres 7,11 et 13). Les déclarations ne concernant pas } \\
\text { des entreprises, ainsi que les déclarations au titre du chapitre } 12 \text { (faillites d'exploitations familiales agricoles et piscicoles) ne sont pas prises en } \\
\text { compte. } \\
\text { http://www.uscourts.gov/ }\end{array}$ \\
\hline Finlande & $\begin{array}{l}\text { Source: Statistics Finland. } \\
\text { Données mensuelles. } \\
\text { Statistiques sur les faillites. } \\
\text { Les données comptabilisent les faillites d'entreprises et de sociétés prononcées par des tribunaux de première instance. } \\
\text { Toutes les activités sont prises en compte. } \\
\text { http://pxnet2.stat.fi/PXWeb/pxweb/en/StatFin/ }\end{array}$ \\
\hline France & $\begin{array}{l}\text { Source: Institut national de la statistique et des études économiques (INSEE) et Banque de France. } \\
\text { Données mensuelles. } \\
\text { Défaillances d'entreprises. } \\
\text { Une défaillance d'entreprise désigne l'ouverture d'une procédure d'insolvabilité. Les statistiques sur les défaillances d'entreprises couvrent } \\
\text { l'ensemble des jugements prononçant soit l'ouverture d'une procédure de redressement judiciaire, soit la liquidation judiciaire. Elles ne rendent pas } \\
\text { compte de l'issue de la procédure : poursuite de l'activité, rachat ou liquidation. } \\
\text { http://www.insee.fr }\end{array}$ \\
\hline Islande & $\begin{array}{l}\text { Source: Statistics Iceland. } \\
\text { Données mensuelles. } \\
\text { Les données concernent les entreprises islandaises en état de cessation de paiements, et proviennent du registre des entreprises de l'Internal } \\
\text { Revenue Directorate. } \\
\text { www.statice.is }\end{array}$ \\
\hline Italie & $\begin{array}{l}\text { Source: Cerved. } \\
\text { Données trimestrielles. } \\
\text { Statistiques sur les faillites. } \\
\text { https://know.cerved.com }\end{array}$ \\
\hline Japon & 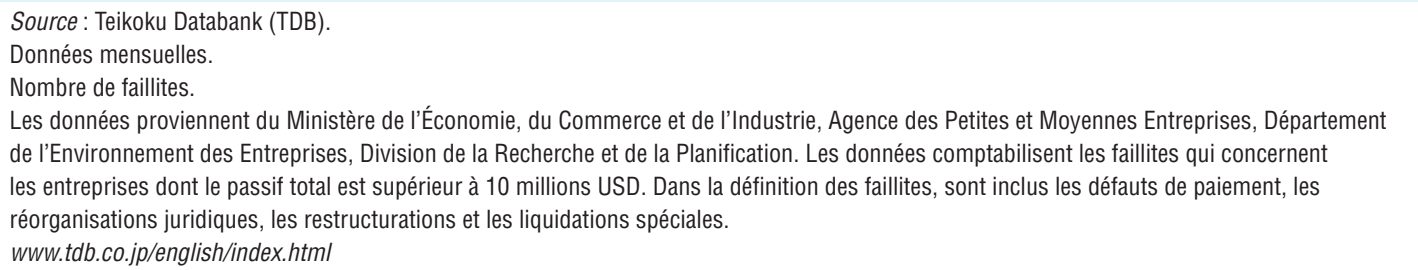 \\
\hline Norvège & $\begin{array}{l}\text { Source: Statistics Norway. } \\
\text { Données mensuelles. } \\
\text { Statistiques sur les faillites. } \\
\text { Les données se réfèrent à l'ensemble de l'économie, sauf l'agriculture. Les entreprises individuelles sont également prises en compte. http:// } \\
\text { statbank.ssb.no }\end{array}$ \\
\hline Nouvelle-Zélande & $\begin{array}{l}\text { Source: New Zealand Companies Office. } \\
\text { Données trimestrielles. } \\
\text { Les données concernent les liquidations, et uniquement pour les entreprises constituées en sociétés. }\end{array}$ \\
\hline Pays-Bas & $\begin{array}{l}\text { Source: Statistics Netherlands. } \\
\text { Données mensuelles. } \\
\text { Nombre de faillites prononcées par les tribunaux néerlandais. Les données se réfèrent à l'ensemble de l'économie, dont l'agriculture, et incluent les } \\
\text { faillites de sociétés ou d'institutions (mais pas des entreprises individuelles). } \\
\text { http://statline.cbs.nl }\end{array}$ \\
\hline Royaume-Uni & $\begin{array}{l}\text { Source: Companies House. } \\
\text { Données mensuelles. } \\
\text { Entreprises constituées en société uniquement. } \\
\text { Les données concernent les liquidations, dont les liquidations judiciaires, les liquidations volontaires à la demande des créanciers et les arrêtés } \\
\text { administratifs convertis en liquidations volontaires à la demande des créanciers. Sont exclues les liquidations volontaires à la demande des } \\
\text { membres. } \\
\text { www.companieshouse.gov.uk/ }\end{array}$ \\
\hline Suède & $\begin{array}{l}\text { Source: Swedish Agency for Growth Policy Analysis. } \\
\text { Données mensuelles. } \\
\text { Statistiques sur les faillites. } \\
\text { Les données correspondent aux faillites d'entreprises, y compris les commerçants indépendants, prononcées par des tribunaux de première } \\
\text { instance. } \\
\text { Toutes les activités sont prises en compte. } \\
\text { www.tillvaxtanalys.se }\end{array}$ \\
\hline
\end{tabular}


ANNEXE B

\section{Liste des indicateurs des déterminants entrepreneuriaux}

La présente annexe dresse une liste complète d'indicateurs des déterminants entrepreneuriaux. Les indicateurs sont regroupés dans les six catégories de déterminants établies par le Programme d'indicateurs de l'entrepreneuriat OCDE-Eurostat : 1 . Cadre réglementaire ; 2 . Situation du marché ; 3 . Accès aux financements ; 4 . Création et diffusion de connaissances ; 5 . Capacités entrepreneuriales ; 6 . Culture entrepreneuriale. Une brève description et la source des données sont fournies pour chaque indicateur.

Bien que de nombreux facteurs cruciaux pour l'entrepreneuriat soient couverts par les indicateurs du tableau ci-après, la liste ne saurait être considérée comme exhaustive. La sélection opérée dépend des données actuellement disponibles, d'importants indicateurs pouvant donc manquer, faute tout simplement de données internationales.

Tableau B.1. Indicateurs des déterminants entrepreneuriaux et sources des données

\begin{tabular}{|c|c|c|}
\hline Catégories de déterminants & Définition & Source de données \\
\hline \multicolumn{3}{|c|}{ 1. CADRE RÉGLEMENTAIRE } \\
\hline \multicolumn{3}{|c|}{ Charge administrative (entrée et croissance) } \\
\hline $\begin{array}{l}\text { Charge de la réglementation } \\
\text { administrative }\end{array}$ & $\begin{array}{l}\text { Réponses issues d'enquêtes sur la question du respect des obligations administratives } \\
\text { (autorisations, réglementation, déclaration) nationales ( } 1 \text { = lourd, } 7 \text { = léger). http://reports.weforum. } \\
\text { org/global-competitiveness-report-2015-2016/competitiveness-rankings/ }\end{array}$ & $\begin{array}{l}\text { Forum économique } \\
\text { mondial, Global } \\
\text { Competitiveness Report }\end{array}$ \\
\hline $\begin{array}{l}\text { Coûts induits par la création d'une } \\
\text { entreprise }\end{array}$ & $\begin{array}{l}\text { Coût officiel de la procédure en pourcentage du revenu national brut (RNB) par habitant, d'après } \\
\text { la législation officielle et des hypothèses classiques concernant les procédures et les entreprises. } \\
\text { http://francais.doingbusiness.org/data/exploretopics/starting-a-business }\end{array}$ & $\begin{array}{l}\text { Banque mondiale, Doing } \\
\text { Business }\end{array}$ \\
\hline $\begin{array}{l}\text { Capital minimum pour créer une } \\
\text { entreprise }\end{array}$ & $\begin{array}{l}\text { Montant minimum libéré de capital - en pourcentage du revenu par habitant - que l'entrepreneur } \\
\text { doit déposer en banque pour que l'immatriculation de son entreprise puisse être déclenchée. } \\
\text { http://francais.doingbusiness.org/data/exploretopics/starting-a-business }\end{array}$ & $\begin{array}{l}\text { Banque mondiale, Doing } \\
\text { Business }\end{array}$ \\
\hline $\begin{array}{l}\text { Nombre de jours nécessaires pour } \\
\text { créer une entreprise }\end{array}$ & $\begin{array}{l}\text { Durée moyenne (en jours calendaires) de la procédure nécessaire pour démarrer une entreprise. } \\
\text { http://francais.doingbusiness.org/data/exploretopics/starting-a-business }\end{array}$ & $\begin{array}{l}\text { Banque mondiale, Doing } \\
\text { Business }\end{array}$ \\
\hline $\begin{array}{l}\text { Nombre de procédures nécessaires } \\
\text { pour créer une entreprise }\end{array}$ & $\begin{array}{l}\text { Toutes les procédures génériques officiellement requises pour l'enregistrement d'une entreprise. } \\
\text { http://francais.doingbusiness.org/data/exploretopics/starting-a-business }\end{array}$ & $\begin{array}{l}\text { Banque mondiale, Doing } \\
\text { Business }\end{array}$ \\
\hline $\begin{array}{l}\text { Durée et coût des procédures de } \\
\text { création d'un entrepôt }\end{array}$ & $\begin{array}{l}\text { Correspond à la moyenne de trois mesures : 1) temps moyen passé pour chaque procédure ; } \\
\text { 2) coût officiel de chaque procédure et 3) nombre de procédures nécessaires pour construire } \\
\text { un entrepôt. http://francais.doingbusiness.org/data/exploretopics/dealing-with-construction-permits }\end{array}$ & $\begin{array}{l}\text { Banque mondiale, Doing } \\
\text { Business }\end{array}$ \\
\hline $\begin{array}{l}\text { Indice de contrôle qualité de la } \\
\text { construction }\end{array}$ & $\begin{array}{l}\text { Cet indicateur est constitué de six indices : la qualité de la réglementation en matière de } \\
\text { construction, le contrôle qualité avant, pendant et après la construction, les régimes de } \\
\text { responsabilité et d'assurance, et la certification professionnelle. } \\
\text { http://francais.doingbusiness.org/methodology/dealing-with-construction-permits }\end{array}$ & $\begin{array}{l}\text { Banque mondiale, Doing } \\
\text { Business }\end{array}$ \\
\hline Enregistrement d'un bien & $\begin{array}{l}\text { Correspond à la moyenne de trois mesures : 1) nombre de procédures obligatoires pour enregistrer } \\
\text { un bien ; 2) temps passé pour chaque procédure et 3) coût d'enregistrement d'un bien. } \\
\text { http://francais.doingbusiness.org/data/exploretopics/registering-property }\end{array}$ & $\begin{array}{l}\text { Banque mondiale, Doing } \\
\text { Business }\end{array}$ \\
\hline $\begin{array}{l}\text { Indice de qualité de l'administration } \\
\text { foncière }\end{array}$ & $\begin{array}{l}\text { L'indice de qualité de l'administration foncière est constitué par la somme des scores obtenus } \\
\text { au titre de quatre indicateurs : la fiabilité des infrastructures, la transparence de l'information, la } \\
\text { couverture géographique et la résolution des litiges fonciers. L'indicateur va de } 0 \text { à } 30 \text {, les valeurs } \\
\text { les plus élevées correspondant à une meilleure qualité du système d'administration foncière. } \\
\text { http://francais.doingbusiness.org/data/exploretopics/registering-property }\end{array}$ & $\begin{array}{l}\text { Banque mondiale, Doing } \\
\text { Business }\end{array}$ \\
\hline
\end{tabular}




\section{Tableau B.1. Indicateurs des déterminants entrepreneuriaux et sources des données (suite)}

\begin{tabular}{|c|c|c|}
\hline Temps passé à payer les impôts & $\begin{array}{l}\text { Temps passé à préparer, déclarer et acquitter l'impôt sur les sociétés, la TVA et les cotisations } \\
\text { sociales. Ce temps est mesuré en nombre d'heures par an. } \\
\text { http://francais.doingbusiness.org/data/exploretopics/paying-taxes }\end{array}$ & $\begin{array}{l}\text { Banque mondiale, Doing } \\
\text { Business }\end{array}$ \\
\hline \multicolumn{3}{|l|}{ Réglementation des faillites } \\
\hline $\begin{array}{l}\text { Coût - Coût moyen des procédures } \\
\text { de faillites }\end{array}$ & $\begin{array}{l}\text { Le coût des procédures est exprimé en pourcentage de la valeur du patrimoine. } \\
\text { http://francais.doingbusiness.org/data/exploretopics/resolving-insolvency }\end{array}$ & $\begin{array}{l}\text { Banque mondiale, Doing } \\
\text { Business }\end{array}$ \\
\hline $\begin{array}{l}\text { Temps - Délai moyen pour la } \\
\text { fermeture d'une entreprise }\end{array}$ & $\begin{array}{l}\text { Le délai de fermeture est exprimé en nombre d'années civiles. II comprend les appels et les retards. } \\
\text { http://francais.doingbusiness.org/data/exploretopics/resolving-insolvency }\end{array}$ & $\begin{array}{l}\text { Banque mondiale, Doing } \\
\text { Business }\end{array}$ \\
\hline Taux de recouvrement & $\begin{array}{l}\text { Le taux de recouvrement calcule combien de centimes par dollar les créanciers bénéficiant d'une } \\
\text { garantie recouvrent sur une entreprise insolvable à l'issue de la procédure d'insolvabilité. } \\
\text { http://francais.doingbusiness.org/data/exploretopics/resolving-insolvency }\end{array}$ & $\begin{array}{l}\text { Banque mondiale, Doing } \\
\text { Business }\end{array}$ \\
\hline
\end{tabular}

\section{Système judiciaire et juridique}

Exécution des contrats - Coût en pourcentage de la créance

Exécution des contrats - Délai

Exécution des contrats - Qualité des procédures judiciaires

\section{Régulation du marché des biens et du marché du travail}

Difficulté de recruter

Difficultés relatives aux licenciements

Degré de facilité d'embauche de salariés étrangers

Indice de la rigidité des heures

Qualité de l'emploi moyens d'avocats. payés annuels.

Le coût est exprimé en pourcentage du montant de la demande, soit l'équivalent de $200 \%$ du en compte. On relève trois types de coûts : les frais de justice, les frais d'exécution et les honoraires

http://francais.doingbusiness.org/data/exploretopics/enforcing-contracts

Nombre de jours calendaires nécessaires pour le règlement d'un différend, comptés à partir du moment où le plaignant engage la poursuite jusqu'au paiement de la réparation. Ce délai comprend à la fois les jours d'audience et les périodes d'attente entre les différentes phases du procès. http://francais.doingbusiness.org/data/exploretopics/enforcing-contracts

L'indicateur de qualité des procédures judiciaires mesure dans chaque pays l'existence d'une série de bonnes pratiques au sein de l'appareil judiciaire et ce, dans quatre domaines : structure des tribunaux et procédures judiciaires, gestion des affaires, automatisation du système judiciaire et modes alternatifs de règlement des litiges.

http://francais.doingbusiness.org/data/exploretopics/enforcing-contracts

Mesure si le droit ou la réglementation entrave ou non la faculté pour une entreprise standard d'embaucher un salarié standard. Cet indicateur évalue notamment dans quelle mesure les contrats à durée déterminée sont interdits pour les tâches permanentes, la durée maximale cumulée des contrats à durée déterminée, le rapport entre le salaire minimum obligatoire et la valeur ajoutée moyenne par salarié ou la disponibilité de mesures incitatrices accordées aux employeurs pour embaucher des employés de moins de 25 ans. http://francais.doingbusiness.org/data/exploretopics/labor-market-regulation\#difficultyHiring Mesure si le droit ou la réglementation entrave ou non la faculté pour une entreprise standard de licencier un salarié standard. Cet indicateur évalue notamment quelle est la durée maximale, en mois, de la période d'essai, ou si l'employeur est tenu d'informer une tierce partie (telle qu'un organisme public) pour le licenciement économique d'un seul salarié. http://francais.doingbusiness.org/data/exploretopics/labor-market-regulation\#difficultyFiring L'indicateur s'appuie sur les réponses à une question posée lors d'une enquête visant à mesurer l'efficience du marché du travail. La question était la suivante : « Comment qualifiez-vous la réglementation de votre pays au regard de l'embauche de salariés étrangers ? [1 = Très restrictive ; 7 = Pas du tout restrictive].

http://reports. weforum.org/global-competitiveness-report-2015-2016/appendix-a-measurement-ofkey-concepts-and-preliminary-index-structure/

Cet indicateur est un indice comportant sept volets, dont les plus importants sont : i) le nombre maximal de jours qu'il est permis de travailler dans une semaine ; ii) l'augmentation de salaire pour le travail de nuit ; iii) la présence ou non de restrictions concernant le travail de nuit ; iv) la présence de restrictions ou non concernant le travail les jours fériés en semaine ; v) la durée des congés

http://francais.doingbusiness.org/data/exploretopics/labor-market-regulation\#rigidityHours L'indicateur évalue 12 aspects : (i) si la loi exige la rémunération égale pour des tâches de mêmes valeurs ; (ii) si la loi exige la non-discrimination fondée sur le sexe dans le cadre de l'embauche ; (iii) si la loi exige des congés maternité payés ou non payés ; (iv) la durée minimum des congés maternité (exprimés en jours calendaires) ; (v) si les employés en congé de maternité bénéficient de $100 \%$ de leur paye ; (vi) la disponibilité de cinq jours de congés maladie entièrement rémunérés ; (vii) la disponibilité d'une formation au sein de l'entreprise sans frais pour l'employé ; (viii) si un travailleur est éligible pour un programme de protection contre le chômage après un an de service ; (ix) la durée minimum, en mois, de la période de contribution requise pour la protection contre le chômage ; (x) si un employé peut créer ou se joindre à un syndicat ; (xi) la disponibilité de recours administratifs ou judiciaires en cas de transgression des droits de l'employé ; et (xii) la disponibilité d'un système d'inspection du travail.

http://francais.doingbusiness.org/data/exploretopics/labor-market-regulation\#rigidityEmployment
Banque mondiale, Doing

Business

Banque mondiale, Doing

Business

Banque mondiale, Doing Business

Banque mondiale, Doing Business

Banque mondiale, Doing Business

Forum économique mondial, Executive Opinion Survey

Banque mondiale, Doing Business

Banque mondiale, Doing Business 


\section{Tableau B.1. Indicateurs des déterminants entrepreneuriaux et sources des données (suite)}

Impôts sur le revenu, impôts sur le patrimoine/droits de succession

Impôt moyen sur le revenu +

prélèvements sociaux

Impôt marginal maximal sur le

revenu + prélèvements sociaux

Recettes tirées des droits de

succession

Recettes tirées de l'impôt sur le

patrimoine net

Taux d'imposition des PME

http://stats.oecd.org/Index.aspx?DataSetCode=Table_II2.

Recettes tirées de l'impôt sur les sociétés

En pourcentage du PIB

http://dx.doi.org/10.1787/ctpa-rev-data-fr

Imposition sur les options d'achat

d'actions

d'un célibataire (sans enfant) à hauts revenus.

http://dx.doi.org/10.1787/data-00265-fr d'un célibataire (sans enfant) à hauts revenus.

http://dx.doi.org/10.1787/data-00265-fr

En pourcentage du PIB

http://dx.doi.org/10.1787/ctpa-rev-data-fr

En pourcentage du PIB.

http://dx.doi.org/10.1787/ctpa-rev-data-fr http://dx.doi.org/10.1787/9789264036116-fr
Impôts sur les sociétés et le capital
Taux moyen d'imposition en pourcentage du salaire brut. L'indicateur repose sur le cas standard

Taux maximal d'imposition en pourcentage du salaire brut. L'indicateur repose sur le cas standard

Coin fiscal moyen sur les actions acquises et nouvellement cotées, sur la base des revenus moyens.
OCDE, Statistiques des

recettes publiques

OCDE, Statistiques des recettes publiques

OCDE, Statistiques des recettes publiques

OCDE, Statistiques des recettes publiques

OCDE, Statistiques des recettes publiques

OCDE, Statistiques des recettes publiques

OCDE, « La taxation des options de souscription ou d'achat d'actions destinées aux salarié », Études de politique fiscale, $\mathrm{n}^{\circ} 11$

\section{Systèmes de brevets, normes}

Droits de propriété intellectuelle

Réponses issues d'enquêtes sur la question : quel est le degré de protection de la propriété intellectuelle dans votre pays, en prenant aussi en compte les mesures anti-contrefaçon ? (1 = extrêmement faible, 7 = extrêmement forte)

http://reports. weforum.org/global-competitiveness-report-2015-2016/competitiveness-rankings/

Droits de propriété

Réponses issues d'enquêtes sur la question : dans votre pays, les droits de propriété, y compris sur les actifs financiers sont ( 1 = peu définis et non protégés par la loi, 7 = clairement définis et bien protégés par la loi).

http://reports.weforum.org/global-competitiveness-report-2015-2016/competitiveness-rankings/
Forum économique mondial, Global Competitiveness Report

Forum économique mondial, Global Competitiveness Report

\section{SITUATION DU MARCHÉ}

\section{Accès aux marchés étrangers}

Commerce transfrontalier

Obstacles aux échanges et à

l'investissement

Indice de restrictivité des échanges de services (IRES)

Niveau d'intervention du secteur public

Entreprises et investissements publics

Restrictions à l'obtention de licences

\section{Demande privée}

Sophistication des acheteurs
L'indicateur se compose de deux volets : 1) les délais (en jours) nécessaires pour accomplir toutes les procédures liées aux exportations et importations de marchandises ; 2) les coûts liés à la logistique des exportations et importations de marchandises.

http://francais.doingbusiness.org/data/exploretopics/trading-across-borders

L'indicateur mesure les barrières explicites et les autres barrières aux échanges et aux investissements. II est basé sur des données qualitatives (lois, réglementations) collectées régulièrement et transformées en données quantitatives.

www.oecd.org/eco/growth/indicatorsofproductmarketregulationhomepage.htm\#indicators

L'indicateur est calculé à partir d'une base de données sur les réglementations contenant des informations comparables et standardisées sur les politiques liées aux échanges et aux investissements qui sont en vigueur dans chaque pays.

http://www.oecd.org/fr/tad/echanges-services/indice-restrictivite-echanges-services.htm

Volume, composition et pourcentage de la production issue des entreprises d'État, et investissements publics en proportion de l'ensemble des investissements.

https://www.fraserinstitute.org/sites/default/files/economic-freedom-of-the-world-data-forresearchers.xIs

Des scores de 0 à 10 sont attribués pour i) le coût en temps (nombre de jours civils nécessaires pour obtenir une licence) et ii) le coût financier de l'obtention de la licence (en pourcentage du revenu par habitant). La note finale est la moyenne de ces deux scores.

http://iresearch.worldbank.org/servicetrade/ default.htm\#

Réponses issues d'enquêtes sur les décisions d'achat ( 1 = en fonction du prix seulement, 7 = en fonction d'une analyse complexe de la performance).

http://reports. weforum.org/global-competitiveness-report-2015-2016/competitiveness-rankings/
Banque mondiale, Doing Business

OCDE, Indicateurs de réglementation des marchés de produits

OCDE, Indice de restrictivité des échanges de services, Base de données sur les réglementations

FMI, Banque mondiale, Comptes nationaux des Nations Unies et Forum économique mondial Banque mondiale

\section{ACCÈS AUX FINANCEMENTS}

Accès à l'emprunt

Note de crédit du pays 


\section{Tableau B.1. Indicateurs des déterminants entrepreneuriaux et sources des données (suite)}

\begin{tabular}{|c|c|c|}
\hline Crédit intérieur au secteur privé & $\begin{array}{l}\text { L'indicateur fait référence aux ressources financières apportées au secteur privé (prêts, achats de } \\
\text { titres autres que des participations, crédits commerciaux et autres effets à recevoir) qui créent une } \\
\text { créance. Les données proviennent des statistiques financières internationales du FMI. } \\
\text { http://databank.banquemondiale.org/data/reports.aspx?source=Indicateurs\%20du\%20 } \\
\text { d\%C3\%A9veloppement\%20dans\%20le\%20monde }\end{array}$ & $\begin{array}{l}\text { Publié dans Indicateurs } \\
\text { du développement dans le } \\
\text { monde, Banque mondiale }\end{array}$ \\
\hline Facilité d'accès au crédit & $\begin{array}{l}\text { Indicateur élaboré à partir de réponses à des enquêtes sur la facilité d'obtention d'un prêt bancaire } \\
\text { dans le pays sur seule présentation d'un bon plan prévisionnel et sans garanties de paiement. (1 = } \\
\text { extrêmement difficile, } 7 \text { = extrêmement facile) } \\
\text { http://reports.weforum.org/global-competitiveness-report-2015-2016/competitiveness-rankings/ }\end{array}$ & $\begin{array}{l}\text { Forum économique mondial, } \\
\text { Global Competitiveness } \\
\text { Report }\end{array}$ \\
\hline Écart de taux d'intérêt & $\begin{array}{l}\text { Taux d'intérêt des prêts moins le taux de rémunération des dépôts (sur la base des taux annuels } \\
\text { moyens de chaque pays). } \\
\text { http://donnees.banquemondiale.org/indicateur/FR.INR.LNDP }\end{array}$ & $\begin{array}{l}\text { Les données ouvertes de la } \\
\text { Banque mondiale }\end{array}$ \\
\hline Fiabilité des droits légaux & $\begin{array}{l}\text { Évalue dans quelle mesure les lois sur le nantissement et la faillite facilitent le prêt. Les valeurs } \\
\text { élevées indiquent que les lois sur les sûretés et la faillite sont mieux adaptées au développement de } \\
\text { l'obtention de prêts. } \\
\text { http://francais.doingbusiness.org/data/exploretopics/getting-credit }\end{array}$ & $\begin{array}{l}\text { Banque mondiale, Doing } \\
\text { Business }\end{array}$ \\
\hline $\begin{array}{l}\text { Part des prêts accordés aux } \\
\text { PME dans le total des prêts aux } \\
\text { entreprises }\end{array}$ & $\begin{array}{l}\text { Des définitions spécifiques sont appliquées par les pays figurant dans le Tableau de bord. } \\
\text { http://www.oecd.org/cfe/smes/financing-smes-and-entrepreneurs-23065265.htm }\end{array}$ & $\begin{array}{l}\text { Le financement des PME et } \\
\text { des entrepreneurs : Tableau } \\
\text { de bord de l'OCDE }\end{array}$ \\
\hline $\begin{array}{l}\text { Écart de taux d'intérêt entre le } \\
\text { taux moyen des PME et celui des } \\
\text { grandes entreprises }\end{array}$ & $\begin{array}{l}\text { Des définitions spécifiques sont appliquées par les pays figurant dans le Tableau de bord. } \\
\text { http://www.oecd.org/cfe/smes/financing-smes-and-entrepreneurs-23065265.htm }\end{array}$ & $\begin{array}{l}\text { Le financement des PME et } \\
\text { des entrepreneurs : Tableau } \\
\text { de bord de l'OCDE }\end{array}$ \\
\hline \multicolumn{3}{|l|}{ Accès au capital-risque } \\
\hline Disponibilité du capital-risque & $\begin{array}{l}\text { Réponses issues d'enquêtes sur la facilité, pour des entrepreneurs avec des projets risqués mais } \\
\text { innovants, d'accéder à du capital-risque dans leur pays ( } 1=\text { extrêmement difficile, } 7=\text { extrêmement facile). } \\
\text { http://reports.weforum.org/global-competitiveness-report-2015-2016/competitiveness-rankings/ }\end{array}$ & $\begin{array}{l}\text { Forum économique mondial, } \\
\text { Global Competitiveness } \\
\text { Report }\end{array}$ \\
\hline Capital-risque & Investissements réalisés par des entreprises de capital-risque. & $\begin{array}{l}\text { Base de données de l'OCDE } \\
\text { sur le financement de } \\
\text { l'entrepreneuriat }\end{array}$ \\
\hline \multicolumn{3}{|l|}{ Marchés d'actions } \\
\hline $\begin{array}{l}\text { Capitalisation boursière du marché } \\
\text { primaire }\end{array}$ & $\begin{array}{l}\text { Capitalisation du marché boursier primaire (valeur des actions émises sur le marché) par rapport au PIB. } \\
\text { http://www.world-exchanges.org/home/index.php/statistics/ipo-database }\end{array}$ & $\begin{array}{l}\text { World Federation of } \\
\text { Exchanges }\end{array}$ \\
\hline $\begin{array}{l}\text { Capitalisation boursière du marché } \\
\text { secondaire }\end{array}$ & $\begin{array}{l}\text { Évalue la capacité des marchés d'actions à financer les entreprises. Le classement va de } 1 \text { (pire) à } \\
10 \text { (meilleur). } \\
\text { http://www.imd.org/wcc }\end{array}$ & $\begin{array}{l}\text { IMD, World Competitiveness } \\
\text { Yearbook }\end{array}$ \\
\hline Protection des investisseurs & $\begin{array}{l}\text { Principaux indicateurs : transparence des opérations (indice de publicité des opérations), } \\
\text { responsabilité en cas d'opérations pour compte propre (indice de responsabilité des } \\
\text { administrateurs), possibilité pour les actionnaires de poursuivre les dirigeants et les } \\
\text { administrateurs pour faute (indice de facilité des poursuites pour l'actionnaire). L'indice de } \\
\text { protection des investisseurs est la moyenne des trois indices. } \\
\text { http://francais.doingbusiness.org/data/exploretopics/protecting-minority-investors }\end{array}$ & $\begin{array}{l}\text { Banque mondiale, Doing } \\
\text { Business }\end{array}$ \\
\hline $\begin{array}{l}\text { Capitalisation boursière des } \\
\text { entreprises récemment cotées }\end{array}$ & $\begin{array}{l}\text { Capitalisation boursière (nombre total de nouvelles actions émises, multiplié par la valeur au } \\
\text { premier jour de cotation) des entreprises nouvellement cotées du pays, rapportée au PIB. } \\
\text { http://www.world-exchanges.org/home/index.php/statistics/ipo-database }\end{array}$ & $\begin{array}{l}\text { World Federation of } \\
\text { Exchanges }\end{array}$ \\
\hline
\end{tabular}

\section{CRÉATION ET DIFFUSION DE CONNAISSANCES}

Activité de recherche et développement (R-D)

Dépenses de R-D des entreprises Dépenses de R-D des entreprises aux prix courants et en parités de pouvoir d'achat courantes. http://dx.doi.org/10.1787/msti-v2015-2-table23-fr

Dépenses intérieures brutes en R-D Dépenses brutes de R-D d'un pays comprenant toutes les dépenses effectuées sur le territoire sur (DIRD)t une période donnée. http://dx.doi.org/10.1787/msti-v2015-2-table12-fr

Dépenses intramuros de R-D de Dépenses intramuros de R-D du secteur de l'enseignement supérieur (DIRDES) aux prix et PPA de 2010. l'enseignement supérieur (DIRDES) http://dx.doi.org/10.1787/msti-v2015-2-table45-fr

Coopération internationale en matière de dépôts de demande de brevet au titre du PCT

Brevets accordés

Transfert des connaissances non commerciales

Recherche de l'enseignement supérieur financée par les entreprises
Mesure la coopération internationale qui se met en place entre les demandes de brevets dans le cadre du Traité de coopération en matière de brevets (PCT). La mesure est établie en pourcentage du total des brevets (en fonction de la date de dépôt de la demande). http://dx.doi.org/10.1787/data-00507-fr

Nombre de brevets accordés aux inventeurs, en fonction de leur lieu de résidence. Somme des brevets octroyés par l'OEB (Office européen des brevets) et l'USPTO (US Patent and trademark office). http://dx.doi.org/10.1787/data-00507-fr

Dépenses de R-D effectuées par l'enseignement supérieur et financées par les entreprises, mesurées en USD de 2010, à prix et PPA .constants. http://dx.doi.org/10.1787/data-00189-fr
OCDE, Principaux indicateurs de la science et de la technologie OCDE, Principaux indicateurs de la science et de la technologie

OCDE, Principaux indicateurs de la science et de la technologie Statistiques de l'OCDE sur les brevets

Statistiques de l'OCDE sur les brevets science et technologie 


\section{Tableau B.1. Indicateurs des déterminants entrepreneuriaux et sources des données (suite)}

Brevets détenus par des universités Brevets détenus par des universités ou des laboratoires publics, par point de PIB. Seuls les pays ou des laboratoires publics

Universités ou autres organismes publics de recherche à l'origine d'innovations

Collaboration entre universités et entreprises en matière de R-D

\section{Coopération inter-entreprises}

PME collaborant avec d'autres entreprises pour l'innovation

Disponibilité et adoption des technologies

Chiffre d'affaires du commerce en ligne

Entreprises utilisant

l'administration électronique

Dépenses de TIC

communications

où existent plus de 250 brevets pendant la période étudiée sont représentés http://dx.doi.org/10.1787/d3bb1fb8-fr

Part des entreprises innovantes qui déclarent que les universités ou d'autres organismes publics de recherche représentent une source importante d'innovation.

Réponses issues d'enquêtes sur le niveau de collaboration existant entre les entreprises et les universités en matière de R-D (de 1 pour inexistante à 7 pour intensive). http://reports.weforum.org/global-competitiveness-report-2015-2016/competitiveness-rankings/

Part des PME innovantes déclarant la coopération comme source d'innovation.

Total des ventes par internet durant l'année civile passée, hors TVA, en pourcentage du chiffre d'affaire total.

http://ec.europa.eu/eurostat/tgm/table. do?tab=table\&init=1\&language $=$ fr \&pcode $=$ tin00110\&plugin $=1$

Part des entreprises qui utilisent un service d'administration électronique parmi toutes les entreprises d'au moins 10 salariés, hors secteur financier.

http://appsso.eurostat.ec.europa.eu/nui/show.do?dataset=isoc_bde15ee\&long=fr

Dépenses d'équipements, de logiciels et de services de TIC, en pourcentage du PIB. http://appsso. eurostat.ec.europa.eu/nui/show. do?dataset=isoc tc ite\&lang=fr

Dépenses d'équipements et de services de télécommunication, en pourcentage du PIB. http:// appsso.eurostat.ec.europa.eu/nui/show.do?dataset=isoc_tc_ite\&lang=fr

Perspectives de l'OCDE de la science, la technologie et l'industrie

Enquêtes (nationales) sur

l'innovation

Forum économique mondial Global Competitiveness Report

Enquêtes (nationales) sur l'innovation

Eurostat, Statistiques sur la société de l'information

Eurostat, Statistiques sur la société de l'information

Observatoire européen des technologies de l'information (EITO)

Observatoire européen des technologies de l'information (EITO)

\section{CAPACITÉS ENTREPRENEURIALES}

\section{Formation à l'entrepreneuriat}

Proportion de diplômés de l'enseignement supérieur

Qualité des écoles de gestion

Formation à la création d'entreprise

\section{Immigration}

Migrant ayant un niveau d'études supérieures
Pourcentage d'individus âgés de 25 à 34 ans possédant un diplôme de l'enseignement supérieur, notamment un doctorat ou équivalent.

http://dx.doi.org/10.1787/eag-2015-table8-en

Réponses issues d'enquêtes sur la qualité des écoles nationales de gestion (de 1 = extrêmement mauvaise, parmi les plus mauvaises du monde ; 7 = parmi les meilleures du monde). http://reports. weforum.org/global-competitiveness-report-2015-2016/competitiveness-rankings/

Part de la population âgée de 18 à 64 ans formée à la création d'entreprise pendant ou après les études. A Global Perspective on Entrepreneurship Education and Training (2008). http://www.gemconsortium.org/report

Part des migrants très qualifiés parmi l'ensemble des migrants.

http://www.oecd.org/fr/els/mig/basededonneessurlesimmigresdanslespaysdelocdedioc.htm
OCDE, Panorama de

l'éducation

Forum économique mondial, Global Competitiveness

Report

Global Entrepreneurship

Monitor (GEM)

OCDE, Base de données sur les immigrés dans les pays de l'OCDE

\section{CULTURE ENTREPRENEURIALE}

Valorisation de la réussite entrepreneuriale

Intentions entrepreneuriales

Souhait de devenir un travailleur indépendant

Opinion au sujet des entrepreneurs

Crainte de l'échec

Risque d'échec

Deuxième chance pour les entrepreneurs
Part des 18-64 ans qui considèrent que, dans leur pays, les entrepreneurs bénéficient d'un statut social élevé.

http://Www.gemconsortium.org/

Part des 18-64 ans (hors individus engagés dans une quelconque phase d'activité entrepreneuriale) Global Entrepreneurship ayant l'intention de créer une entreprise dans les trois ans. http://www.gemconsortium.org/

Réponses issues d'enquêtes sur le souhait de devenir un travailleur indépendant dans les 5 années à venir. L'enquête ne s'adresse qu'aux individus qui ne sont pas des travailleurs indépendants. http://ec.europa.eu/public_opinion/flash/fl_354_en.pdf

Réponses issues de sondages d'opinion sur l'image que donnent les entrepreneurs (travailleurs indépendants, chefs d'entreprises). Le classement inclut les dirigeants des grandes entreprises et les professions libérales.

http://ec.europa.eu/public opinion/flash/fl 354 en.pdf

Pourcentage des 18-64 ans non engagés dans une activité entrepreneuriale et qui perçoivent de bonnes opportunités de création d'entreprise, mais que la peur de l'échec empêche de passer à l'acte. http://www.gemconsortium.org/

Réponses issues d'enquêtes sur la propension à créer une entreprise s'il existe un risque d'échec. http://ec.europa.eu/public_opinion/flash/fl_354_en.pdf

Réponses issues d'enquêtes d'opinions sur le caractère opportun de donner une deuxième chance aux personnes qui ont monté leur entreprise mais qui ont échoué.

http://ec.europa.eu/public_opinion/flash/fl_354_en.pdf
Global Entrepreneurship Monitor (GEM)

Monitor (GEM)

Commission européenne, Flash Eurobarometer

Commission européenne, Flash Eurobarometer

Global Entrepreneurship Monitor (GEM)

Commission européenne, Flash Eurobarometer

Commission européenne, Flash Eurobarometer 
ANNEXE C

\section{Comparabilité internationale des données sur le capital-risque}

Les données agrégées sur le capital-risque fournissent des informations utiles sur l'évolution du secteur du capital-risque. Ces données sont généralement compilées par des associations nationales et régionales de capital-investissement et de capital-risque, souvent avec le concours de fournisseurs de données sur les entreprises. La qualité et la disponibilité de données agrégées sur le capital-risque se sont considérablement améliorées ces dernières années ; il demeure difficile, cependant, de réaliser des comparaisons internationales du fait de deux problèmes principaux.

La première difficulté tient à l'absence de définition internationale normalisée du capitalrisque. Si tout le monde comprend de quoi il s'agit, la définition des types d'investissements inclus dans le capital-risque varie d'un pays et d'une région à l'autre. Dans certains cas, les différences sont purement linguistiques ; dans d'autres, elles sont plus profondes.

Le second problème a trait à la diversité des méthodes employées par ceux qui compilent les données. C'est la manière dont les données sont collectées qui détermine si les statistiques sur le capital-risque donneront une image complète et représentative de ce secteur dans un pays donné.

Les tableaux suivants illustrent les différences concernant respectivement : les définitions du capital-investissement et du capital-risque (tableau C.1) ; la ventilation du capital-risque par stade (tableau C.2) ; la ventilation du capital-risque par secteur (tableau C.3) ; enfin, les méthodes de collecte des données (tableau C.4).

Les sources de capital-risque examinées comprennent:

Australian Bureau of Statistics (ABS), Venture Capital and Later Stage Private Equity.

CVCA - Association canadienne du capital de risque et d'investissement.

Invest Europe, Invest Europe Yearbook.

KVCA - Korean Venture Capital Association.

NVCA/PitchBook - National Venture Capital Association/PitchBook, États-Unis, rapport trimestriel.

NZVCA - New Zealand Private Equity and Venture Capital Association.

PwC MoneyTree, Israël.

RVCA - Russian Venture Capital Association.

SAVCA - South African Venture Capital and Private Equity Association/KPMG.

VEC - Venture Enterprise Center, Japon 
Tableau C.1. Définitions du capital-investissement et du capital-risque

\begin{tabular}{|c|c|c|}
\hline Source & Capital-investissement & Capital-risque \\
\hline Invest Europe & $\begin{array}{l}\text { Le capital-investissement correspond à des fonds propres fournis à } \\
\text { des entreprises non cotées en bourse. }\end{array}$ & $\begin{array}{l}\text { Le capital-risque est un segment du secteur du capital- } \\
\text { investissement et désigne les investissements en fonds propres } \\
\text { réalisés pour financer les phases de prédémarrage, de démarrage et } \\
\text { de développement initial d'une entreprise. }\end{array}$ \\
\hline $\begin{array}{l}\text { National Venture Capital } \\
\text { Association-États-Unis } \\
\text { (NVCA)/PitchBook }\end{array}$ & $\begin{array}{l}\text { Le capital-investissement correspond à l'investissement de fonds } \\
\text { propres dans des entreprises non publiques, généralement définies } \\
\text { comme étant constituées de fonds de capital-risque. L'immobilier, } \\
\text { le pétrole, le gaz et tout autre partenariat de ce type sont parfois } \\
\text { comprises dans cette définition. }\end{array}$ & $\begin{array}{l}\text { Le capital-risque est un segment du secteur du capital- } \\
\text { investissement qui consiste avant tout à investir dans de nouvelles } \\
\text { entreprises présentant un fort potentiel de croissance associé à des } \\
\text { risques élevés. }\end{array}$ \\
\hline $\begin{array}{l}\text { Australian Bureau of } \\
\text { Statistics (ABS) }\end{array}$ & $\begin{array}{l}\text { Le capital-investissement (investi à un stade ultérieur) correspond à } \\
\text { des investissements réalisés dans des entreprises ayant atteint des } \\
\text { stades ultérieurs de développement, ainsi qu'à des investissements } \\
\text { réalisés dans des entreprises peu performantes. Ces entreprises } \\
\text { continuent d'être créées, les risques demeurent élevés et les } \\
\text { investisseurs adoptent une stratégie de sortie - où le rendement sur } \\
\text { investissement prend principalement la forme de plus-values -, et } \\
\text { non une stratégie d'investissement à long terme donnant lieu à des } \\
\text { flux de revenus réguliers. }\end{array}$ & $\begin{array}{l}\text { Le capital-risque correspond à du capital-investissement à haut } \\
\text { risque investi dans des entreprises non cotées, généralement } \\
\text { nouvelles, innovantes ou à croissance rapide. Un investissement } \\
\text { de capital-risque est généralement un investissement de court à } \\
\text { moyen terme assorti d'une stratégie de sortie - où le rendement sur } \\
\text { investissement prend principalement la forme de plus-values -, et } \\
\text { non d'une stratégie d'investissement à long terme donnant lieu à des } \\
\text { flux de revenus réguliers. }\end{array}$ \\
\hline $\begin{array}{l}\text { Association canadienne } \\
\text { du capital de risque et } \\
\text { d'investissement (CVCA) }\end{array}$ & $\begin{array}{l}\text { Terme générique pour le marché privé, qui désigne toutes les } \\
\text { formes de participations ou quasi-participations. Dans un secteur } \\
\text { du capital-investissement arrivé à maturité, le marché se subdivise } \\
\text { généralement en trois segments distincts : le capital de rachat, le } \\
\text { financement mezzanine et le capital-risque. }\end{array}$ & $\begin{array}{l}\text { Cette forme particulière de capital-investissement se caractérise } \\
\text { surtout par un investissement à haut risque dans des entreprises } \\
\text { jeunes ou de création récente qui suivent une trajectoire de } \\
\text { croissance. }\end{array}$ \\
\hline $\begin{array}{l}\text { Korean Venture Capital } \\
\text { Association (KVCA) }\end{array}$ & $\begin{array}{l}\text { Le capital-investissement correspond à une méthode de } \\
\text { participation financière avec des capitaux mobilisés par moins de } \\
49 \text { sociétés commanditaires. Cette opération consiste à prendre } \\
\text { une participation majoritaire dans la société dans laquelle est réalisé } \\
\text { l'investissement, à accroître la valeur de cette dernière, puis à } \\
\text { obtenir une plus-value sur la vente des parts. }\end{array}$ & $\begin{array}{l}\text { Investissement réalisé par une société/un fonds dans des entreprises } \\
\text { présentant un fort potentiel et une forte croissance à leur stade de } \\
\text { développement initial. }\end{array}$ \\
\hline $\begin{array}{l}\text { Venture Enterprise } \\
\text { Center - Japon (VEC) }\end{array}$ & $\begin{array}{l}\text { Le capital-investissement est une méthode d'investissement selon } \\
\text { laquelle les investisseurs participent à la gestion et à la gouvernance } \\
\text { d'entreprises afin d'en accroître la valeur en leur apportant les fonds } \\
\text { nécessaires, à différents stades de développement et dans différents } \\
\text { environnements. }\end{array}$ & $\begin{array}{l}\text { Fonds fournis sous forme notamment d'actions, d'obligations } \\
\text { convertibles et de bons de souscription aux sociétés à capital-risque, } \\
\text { qui sont de petites et moyennes entreprises fermées (non publiques) } \\
\text { présentant un potentiel de croissance. }\end{array}$ \\
\hline
\end{tabular}

Tableau C.2. Ventilation du capital-risque par stade, échantillon d'associations de capital-risque et OCDE

\begin{tabular}{|c|c|c|c|c|c|c|c|c|c|c|c|c|}
\hline \multicolumn{3}{|r|}{ Invest Europe } & \multirow[t]{2}{*}{$\begin{array}{c}\text { NVCA/ } \\
\text { PitchBook }\end{array}$} & \multirow[t]{2}{*}{$\begin{array}{l}\text { PwC Money } \\
\text { Tree - Israël }\end{array}$} & \multirow{2}{*}{\begin{tabular}{|c|} 
ABS - \\
Australie \\
Préamorçage
\end{tabular}} & \multirow[t]{2}{*}{ CVCA } & \multirow[t]{2}{*}{ VEC } & \multirow[t]{2}{*}{ KVCA } & \multirow[t]{2}{*}{ NZVCA } & \multirow[t]{2}{*}{ RVCA } & \multirow[t]{2}{*}{ SAVCA } & \multirow[b]{3}{*}{$\begin{array}{c}\text { Préamorçage/ } \\
\text { Amorçage }\end{array}$} \\
\hline \multirow{8}{*}{ 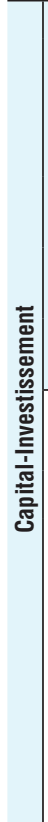 } & \multirow{5}{*}{ 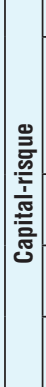 } & & & & & & & & & & & \\
\hline & & Amorçage & \begin{tabular}{|c|} 
Tutorat- \\
investissement \\
(Angel)/ \\
Amorçage
\end{tabular} & \multirow[t]{2}{*}{$\begin{array}{l}\text { Amorçage/ } \\
\text { Démarrage }\end{array}$} & Amorçage & Amorçage & Amorçage & $\begin{array}{l}\text { Stade } \\
\text { initial }\end{array}$ & \multirow[t]{2}{*}{$\begin{array}{l}\text { Amorçage/ } \\
\text { Démarrage }\end{array}$} & \multirow[t]{2}{*}{$\begin{array}{l}\text { Amorçage/ } \\
\text { Démarrage }\end{array}$} & Amorçage & \\
\hline & & Démarrage & \multirow{2}{*}{ Stade initial } & & Dám & Démarrage & $\begin{array}{l}\text { Stade } \\
\text { initial }\end{array}$ & \multirow{3}{*}{ Expansion } & & & \multirow{3}{*}{$\begin{array}{c}\text { Démarrage et } \\
\text { stade initial }\end{array}$} & \multirow{2}{*}{$\begin{array}{c}\text { Démarrage/ } \\
\text { Autre stade } \\
\text { initial }\end{array}$} \\
\hline & & $\begin{array}{l}\text { Autre stade } \\
\text { initial }\end{array}$ & & \begin{tabular}{|c|} 
Stade initial/ \\
Expansion
\end{tabular} & Demarrage & $\begin{array}{c}\text { Autre stade } \\
\text { initial }\end{array}$ & Expansion & & $\begin{array}{l}\text { Expansion } \\
\text { initiale }\end{array}$ & \multirow{2}{*}{$\begin{array}{c}\text { Autres stades } \\
\text { initiaux }\end{array}$} & & \\
\hline & & Stade ultérieur & Stade ultérieur & $\begin{array}{c}\text { Stade } \\
\text { ultérieur }\end{array}$ & $\begin{array}{c}\text { Expansion } \\
\text { initiale }\end{array}$ & Expansion & $\begin{array}{c}\text { Stade } \\
\text { ultérieur }\end{array}$ & & Expansion & & & Stade ultérieur \\
\hline & \multirow{3}{*}{ 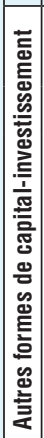 } & \multirow[b]{3}{*}{$\begin{array}{c}\text { Croissance/ } \\
\text { Renflouement/ } \\
\text { Retournement, } \\
\text { Remplacement, } \\
\text { Transmission }\end{array}$} & \multirow[b]{3}{*}{$\begin{array}{l}\text { Capital- } \\
\text { transmission } \\
\text { et capital- } \\
\text { mezzanine }\end{array}$} & \multirow{3}{*}{\multicolumn{2}{|c|}{\begin{tabular}{|} 
Expansion \\
ultérieure, \\
Retournement, \\
Opération \\
de capital- \\
transmission/ \\
Rachat \\
interne/ \\
Rachat par \\
des cadres \\
extérieurs
\end{tabular}}} & \multirow{3}{*}{$\begin{array}{l} \\
\text { Acquisition/ } \\
\text { Transmission, } \\
\text { Retournement, } \\
\text { Autre stade }\end{array}$} & & \multirow[b]{3}{*}{$\begin{array}{c}\text { Stade } \\
\text { ultérieur }\end{array}$} & Retournement & Expansion & $\begin{array}{c}\text { Expansion et } \\
\text { développement }\end{array}$ & \multirow[b]{3}{*}{$\begin{array}{c}\text { Autres formes } \\
\text { de capital- } \\
\text { investissement }\end{array}$} \\
\hline & & & & & & & & & & Restructuration & & \\
\hline & & & & & & & & & $\begin{array}{c}\text { Capital- } \\
\text { investissement } \\
\text { investi dans } \\
\text { des entreprises } \\
\text { moyennes, } \\
\text { Transmission }\end{array}$ & $\begin{array}{c}\text { Stade } \\
\text { ultérieur }\end{array}$ & $\begin{array}{l}\text { Remplacement, } \\
\text { Transmission }\end{array}$ & \\
\hline
\end{tabular}

Note: le CVCA classe l'expansion dans les «Autres formes de capital-investissement ». La NZVCA place le retournement dans le « Capital-risque ». 


\section{Tableau C.3. Ventilation du capital-risque par secteur, Europe et États-Unis}

\begin{tabular}{|c|c|c|}
\hline Classification de l'OCDE & États-Unis - NVCA/PitchBook & Europe - Invest Europe \\
\hline $\begin{array}{l}\text { TIC (Technologies de l'information et de la } \\
\text { communication) }\end{array}$ & Technologies de l'information & TIC (Informatique, électronique et communication) \\
\hline Sciences de la vie & Santé & Biotechnologie et santé \\
\hline Industrie/Énergie & $\begin{array}{l}\text { Énergie } \\
\text { Matières premières et ressources }\end{array}$ & $\begin{array}{l}\text { Énergie et environnement } \\
\text { Produits chimiques et matériaux }\end{array}$ \\
\hline Autres & $\begin{array}{l}\text { Commerce direct avec le particulier (B2C-Business to } \\
\text { consumer) } \\
\text { Commerce interentreprises (B2B-Business to business) } \\
\text { Services financiers }\end{array}$ & $\begin{array}{l}\text { Biens de consommation et services aux } \\
\text { consommateurs } \\
\text { Produits et services destinés aux entreprises } \\
\text { Activités financières et d'assurance } \\
\text { Agriculture } \\
\text { Immobilier } \\
\text { Construction } \\
\text { Transports } \\
\text { Autre }\end{array}$ \\
\hline
\end{tabular}

\section{Tableau C.4. Méthodes de collecte des données sur le capital-risque}

ABS Recensement du capital-risque et du capital-développement domiciliés en Australie et identifiés par l'Australian Bureau of Statistics. Les investissements réalisés par des fonds non-résidents dans des sociétés bénéficiaires australiennes n'entrent pas dans le champ de l'enquête ; cependant, les fonds provenant de non-résidents et les fonds australiens investis dans des entreprises non résidentes en font partie.

CVCA Enquêtes trimestrielles réalisées par Thomson Reuters auprès des gestionnaires de fonds de capital-investissement actifs dans les entreprises canadiennes. Le taux de participation des entreprises est présenté comme très élevé.

Invest Europe Pour le rapport 2016 sur le capital-investissement européen, une nouvelle base de données, "European Data Cooperative » (EDC), a remplacé l'ancienne base PEREP_Analytics. Toutes les données historiques pertinentes ont été transférées dans le système EDC. Toutes les données depuis 2007 ont été retraitées et complétées par des informations supplémentaires. Tous les exercices d'audit sont effectués en étroite coopération avec les contributeurs de données et les associations nationales partenaires pour garantir la meilleure couverture et l'application cohérente de la méthodologie et des définitions.

Invest Europe et ses associations nationales partenaires ont développé l'EDC afin de recueillir à l'échelle européenne des données sur l'activité de l'industrie concernant les levées de fonds, les investissements et les cessions d'actifs. L'EDC reste une base de données à caractère non-lucratif sur le capital-investissement paneuropéen. Elle dispose de son propre personnel et de ses propres ressources grâce aux associations qui la détiennent et l'exploitent. Son approche est basée sur le recensement des sociétés de capital-investissement et de capital-risque européennes identifiées par Invest Europe et ses associations partenaires de capital-investissement et de capital-risque. Les entreprises sont interrogées chaque semestre ou chaque année. Pendant toute la période de collecte de données, les associations prennent contact avec les non-répondants pour les inciter à participer à l'enquête. Les informations peuvent être complétées par des données provenant de sources publiques (comme la presse, les médias, des sites Internet de sociétés de capital-investissement et de capital-risque ou de leurs sociétés en portefeuille) ; les données sont incluses si elles respectent les règles définissant les participants remplissant les conditions requises, la date de transaction, les montants appropriés et les paramètres qualitatifs. Deux sources publiques indépendantes sont généralement exigées pour que les informations puissent être ajoutées à la base de données.

KVCA Recensement des sociétés de capital-risque coréennes enregistrées (pour l'enregistrement, le capital d'une société de capital-risque doit être supérieur à $5000 \mathrm{KRW}$ ). La loi stipule que les sociétés de capital-risque doivent rendre compte de leurs activités tous les mois.

NVCA/PitchBook Rapport trimestriel NVCA/PitchBook : analyse trimestrielle des activités d'investissement de capital-risque aux États-Unis, produite par la NVCA en coopération avec PitchBook. Le rapport inclut les activités d'investissement (dans des entreprises bénéficiaires domiciliées aux États-Unis) de société de capital-risque professionnelles disposant ou non de bureaux aux États-Unis, de sociétés d'investissement spécialisées dans les petites entreprises, de filiales de grandes entreprises spécialisées dans le capital-risque, d'institutions, de banques d'investissement et d'entités analogues dont l'activité principale consiste à financer des investissements. Le tutorat-investissement, les investissements dans des pépinières d'entreprises et autres investissements qui font partie d'un cycle de capital-risque sont inclus s'ils se font via la fourniture de fonds propres liquides et non sous forme de transmission ou de services en nature. Les données sont obtenues principalement par le biais d'une enquête trimestrielle menée par PitchBook auprès de ceux qui pratiquent le capital-risque. Les informations sont complétées grâce à d'autres techniques de recherche comprenant des sources publiques et privées supplémentaires. Toutes les informations font l'objet d'une vérification auprès des sociétés de capital-risque et/ou des entreprises bénéficiaires des investissements.

NZVCA Enquête auprès de ceux qui pratiquent le capital-risque et le capital-investissement sur le marché néo-zélandais, réalisée par la NZVCA et Ernst \& Young. L'enquête porte sur des entreprises situées tant en Nouvelle-Zélande qu'en Australie (l'échantillon de 2011 comprenait 21 répondants). Sont incluses également toutes les informations rendues publiques (S\&P Capital IQ; publication Young Company Finance du New Zealand Venture Investment Fund). La NZVCA et Ernst \& Young reconnaissent qu'un petit nombre des participants du secteur choisissent de ne pas participer à cette enquête.

Israël/PwC Rapport MoneyTree ${ }^{\mathrm{TM}}$ : étude trimestrielle réalisée par PwC Israël.

RVCA Enquête sur les fonds de capital-investissement et de capital-risque actifs sur le marché russe, complétée par les renseignements issus d'entretiens avec des spécialistes du secteur et de sources publiques. En 2012, l'examen des données a concerné plus de 180 fonds. La RVCA considère que les chiffres totaux collectés rendent fidèlement compte des tendances du marché russe.

SAVCA Enquête sur les participants au secteur du capital-investissement, réalisée par KPMG et SAVCA. Les investissements sont inclus s'ils sont réalisés en Afrique du Sud, quel que soit le lieu depuis lequel ils sont gérés. Le capital-investissement investi par des entreprises, des banques et des institutions de financement du développement est pris en compte. En 2012, l'enquête a permis d'obtenir 95 réponses représentant 102 fonds ; des renseignements issus de 15 entreprises supplémentaires de capital-investissement, représentant 15 fonds, ont été ajoutés en puisant à d'autres sources. KPMG et SAVCA estiment que l'enquête représente, en termes de fonds sous gestion, plus de $90 \%$ du secteur sud-africain du capital-investissement. Enquête sur les investisseurs de capital-risque identifiés par le VEC. 



\section{ORGANISATION DE COOPÉRATION ET DE DÉVELOPPEMENT ÉCONOMIQUES}

L'OCDE est un forum unique en son genre où les gouvernements oeuvrent ensemble pour relever les défis économiques, sociaux et environnementaux que pose la mondialisation. L'OCDE est aussi à l'avant-garde des efforts entrepris pour comprendre les évolutions du monde actuel et les préoccupations qu'elles font naître. Elle aide les gouvernements à faire face à des situations nouvelles en examinant des thèmes tels que le gouvernement d'entreprise, l'économie de l'information et les défis posés par le vieillissement de la population. L'Organisation offre aux gouvernements un cadre leur permettant de comparer leurs expériences en matière de politiques, de chercher des réponses à des problèmes communs, d'identifier les bonnes pratiques et de travailler à la coordination des politiques nationales et internationales.

Les pays membres de l'OCDE sont : l'Allemagne, l'Australie, l'Autriche, la Belgique, le Canada, le Chili, la Corée, le Danemark, l’Espagne, l'Estonie, les États-Unis, la Finlande, la France, la Grèce, la Hongrie, l'Irlande, l'Islande, Israël, l'Italie, le Japon, la Lettonie, le Luxembourg, le Mexique, la Norvège, la Nouvelle-Zélande, les Pays-Bas, la Pologne, le Portugal, la République slovaque, la République tchèque, le Royaume-Uni, la Slovénie, la Suède, la Suisse et la Turquie. La Commission européenne participe aux travaux de l'OCDE.

Les Éditions OCDE assurent une large diffusion aux travaux de l'Organisation. Ces derniers comprennent les résultats de l'activité de collecte de statistiques, les travaux de recherche menés sur des questions économiques, sociales et environnementales, ainsi que les conventions, les principes directeurs et les modèles développés par les pays membres. 
Panorama de l'entrepreneuriat 2017

Panorama de l'entrepreneuriat est une publication annuelle qui présente une collection originale d'indicateurs pour mesurer l'état de l'entrepreneuriat accompagnée d'explications sur le contexte politique et l'interprétation des données.

La publication est produite par le Programme d'indicateurs de l'entrepreneuriat OCDE-Eurostat sur la base de données officielles. L'édition 2017 inclut un chapitre sur les nouvelles tendances, qui présente également les développements récents liés à l'émergence des « travailleurs à la demande » et à l'utilisation des outils numériques par les microentreprises.

Veuillez consulter cet ouvrage en ligne : http://dx.doi.org/10.1787/entrepreneur_aag-2017-fr.

Cet ouvrage est publié sur OECD iLibrary, la bibliothèque en ligne de l'OCDE, qui regroupe tous les livres, périodiques et bases de données statistiques de l'Organisation.

Rendez-vous sur le site www.oecd-ilibrary.org pour plus d'informations.

éditionsOCDE

www.oecd.org/editions

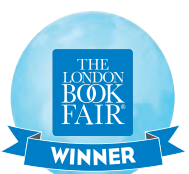

INTERNATIONAL

EXCELLENCE

Awards 2017

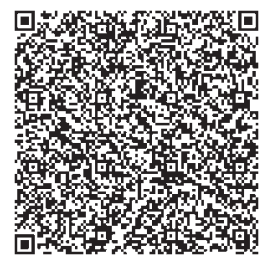

ISBN 978-92-64-28891-1

302017052 P

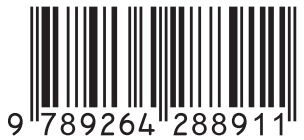

